\title{
Updated Projections of Radioactive Wastes to be Generated by the U.S. Nuclear Power Industry
}
C. W. Kee
A. G. Croff
J. O. Blomeke 


\section{DISCLAIMER}

This report was prepared as an account of work sponsored by an agency of the United States Government. Neither the United States Government nor any agency Thereof, nor any of their employees, makes any warranty, express or implied, or assumes any legal liability or responsibility for the accuracy, completeness, or usefulness of any information, apparatus, product, or process disclosed, or represents that its use would not infringe privately owned rights. Reference herein to any specific commercial product, process, or service by trade name, trademark, manufacturer, or otherwise does not necessarily constitute or imply its endorsement, recommendation, or favoring by the United States Government or any agency thereof. The views and opinions of authors expressed herein do not necessarily state or reflect those of the United States Government or any agency thereof. 


\section{DISCLAIMER}

Portions of this document may be illegible in electronic image products. Images are produced from the best available original document. 
Printed in the United States of America. Available from National Technical Information Service

U.S. Department of Commerce

5285 Port Royal Road, Springfield, Virginia 22161

Price: Printed Copy $\$ 6.00$; Microfiche $\$ 3.00$

This report was prepared as an account of work sponsored by the United States Government. Neither the United States nor the Energy Research and Development Administration/United States Nuclear Regulatory Commission, nor any of their employees, nor any of their contractors, subcontractors, or their employees, makes any warranty, express or implied, or assumes any legal liability or responsibility for the accuracy, completeness or usefulness of any information, apparatus, product or process disclosed, or represents that its use would not infringe privately owned rights. 
Contract No. W-7405-eng-26

CHEMICAL TECHNOLOGY DIVISION

UPDATED PROJECTIONS OF RADIOACTIVE WASTES TO BE

GENERATED BY THE U.S. NUCLEAR POWER INDUSTRY

C. W. Kee

A. G. Croff

J. O. Blomeke

Date Issued: December 1976

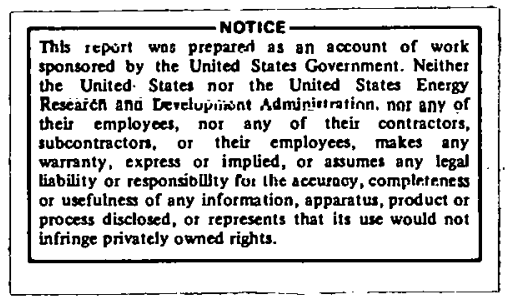

OÁK RIDGE NATIONAL LABOPATORY

Oak Ridge, Tennessee 37830

operated by

UNION CARBIDE CORPORATION

for the

ENERGY RESEARCH AND DEVELOPMENT ADMINISTRATION 
THIS PAGE

\section{WAS INTENTIONALLY LEFT BLANK}


CONTENTS

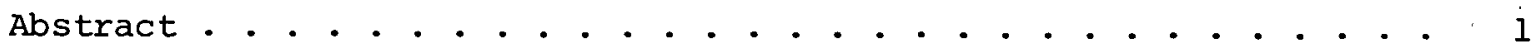

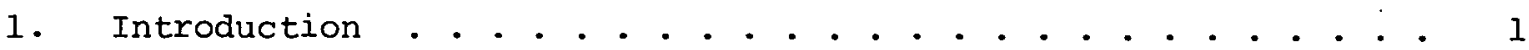

2. Assumed Nuclear Reactor Characteristics . . . . . . . . 4

2.1 Light water Reactors ............. 4

2.2 High-Temperature Gas-Cooled Reactor ......... 9

2.3 A. I. Follow-On LMFBR .............. 9

3. Projections of Nuclear fuel Management .......... 9

4. Origin, Nature, and Projections of Fuel Cycle Wastes ..... 18

4.1 High-Level Wastes .............. 18

4.1.1 Fuel Reprocessing ........... 20

4.1 .2 Waste volumes ............ 21

4.1.3 Radiation Charactcristics of High-Level Wastes . 21

4.1.4 High-Level Waste Projections ........ 22

4.2 Fuel Element Structural Materials (Cladding Wastes) . . . 30

4.3 Intermediate-Level TRU Wastes .......... 43

4.4 Low-Level Transuranic Wastes . . . . . . . . 57

4.5 Noble Gases . . . . . . . . . . . . 69

4.6 Iodine ................ 80

4.7 Carbon-14 . . . . . . . . . . 82

4.8 Tritium ................. 82

4.9 Low-Level Non-TRU Sulid wastes . . . . . . . 86

4.10 Ore Tailings ............. 88

5. References ................ 93 
Table 1.1 Projected nuclear operating capacity, GW(e) ..... 3

Table 1.2 Fuel cycle wastes projected for the year 2000 . . . 5

Table 1.3 Fuel cycle wastes from the production of 1000 MW-years of electricity . . . . . . . . . 6

Table 2.1 Assumed characteristics of light water reactors . . 7

Table 2.2 Assumed characteristics of the HTGR and the A. I. Follow-On LMFBR ............... 8

Table 3.1. Metric tons of U, Th, and Pu consumed, produced, and stockpiled annually ............ 11

Table 3.2. Metric tons of fuel fabricated annually . . . . . 12

Table 3.3' Annual reprocessing load (Metric Tons) . . . . . . . 14

Table 3.4 Fuel reprocessed (Metric Tons) . . . . . . . . 15

Table 3.5 Metric tons of fuel reprocessed annually at rated exposure................. 16

Table 3.6 Number of LWR sfent fuel assemblies discharged . . . . 17

Table 4.1 Comparison of pirincipal assumptions used for waste projections ................ 19

Table 4.2 Radioactivity (MCi) in high-level wastes generated annually ....................

Table 4.3. Thermal power $(\mathrm{kW})$ in high-level wastes generated annually .......................

Table 4.4 Cubic meters of solidified high-level wastes generated annually . . . . . . . . . . . .

Table 4.5 Projected annual accumulations of solidified highlevel wastes without shipment . . . . . . . . .

Table 4.6 Radioactivity (MCi) in 10-year-old high-level wastes shipped annually . . . . . . . . . . .

Table 4.7 Thermal power $(\mathrm{kW})$ in 10-year-old high-level wastes shipped annually ................ 
Table 4.8 Cubic meters of 10-year-old high-level wastes

Table 4.9 Number of 10-year-old high-level waste canisters shipped annually . . . . . . . . . . . . .

Table 4.10 Number of annual 10-year-old high-level waste

shipments . . . . . . . . . . . . . .

Table 4.11. Projected annual accumulation of solidified high-

level waste at a federal repository ... . . . . .

Table 4.12 Grams of accumulated heavy elements in high-level wastes at a.federal repository in the year 2010 as a function of age ................

Table 4.13 Curies of accumulated heavy isotopes in high-level wastes at a federal repository in the year 2010 as a function of age . . . . . . . . . . . . . .

Table 4.14 Watts of accumulated heavy isotopes in high-level wastes at a federal repository in the year 2010 as a function of age. .............. 36

Table $4.15^{-}$Ingestion toxicity $\left(\mathrm{m}^{3}\right.$ water) of accumulated fission product isotopes in high-level wastes at a federal repository in the year 2010 as a function of time..

Table 4.16 Grams of accumulated fission product elements in high-level wastes at a federal repository in the year 2010 as a function of age ...........

Table 4.17 Curies of accumulated fission product isntopee in high-level wastes at a federal repository in the year 2010 as a function of age...........

Table 4.18 Watts of accumulated fission product isotopes in high-level wastes at a federal repository in the year 2010 as a function of age . . . . . . . . .

Table 4.19 Ingestion toxicity $\left(\mathrm{m}^{3}\right.$ water) of accumulated fission product isotopes in high-level wastes at a federal repository in the year 2010 as a function of age...

Table 4.20 Mass, composition, and exposure of fuel assembly structural materials ................

Table 4.21 Projected annual accumulation of cladding waste at. a federal repusitory . . . . . . . . . . . . 
Table 4.22 Grams of accumulated structural materials and impurity elements.in the cladding wastes at a federal repository in the year 2005 as a function of age . . . . . . . .

Table 4.23. Curies of accumulated structural materials and impurity isotopes in the cladding wastes at a federal repository in the year 2005 as a function of age . . . . . .

Table 4.24 Watts of accumulated structural materials and impurity isotopes in the cladding wastes at a federal repository in the year 2005 as a function of age . . . . . . .

Table 4.25 Ingestion toxicity $\left(\mathrm{m}^{3}\right.$ water) of accumulated structural materials and impurity isotopes in the cladding wastes at a federal repository in the year 2005 as a function of age...................

Table 4.26 Grams of accumulated heavy elements in the cladding wastes at a federal repository in the year 2005 as a function of age...................

Table 4.27 Curies of accumulated heavy isotopes in the cladding wastes at a federal repository in the year 2005 as a function of age . . . . . . . . . . . . . . .

Table 4.28 Watts of accumulated heavy isotopes in the cladding wastes at a federal repository in the year 2005 as a Iunctiun of agc. . . . . . . . . . . . . . . .

Table 4.29 Ingestion toxicity ( $\mathrm{m}^{3}$ water) of accumulated heavy isotopes in the cladding wastes at a federal repository in the year 2005 as a function of age . . . . . . .

Table 4.30 Grams of accumulated fission product elements in the cladding wastes at a federal repository in the year 2005 as a function of age..............

Table 4.31 Curies of accumulated fission product isotopes in the cladding wastes at a federal repository in the year 2005 as a function of age . . . . . . . . . . .

Table 4.32 Watts of accumulated fission product isotopes in the cladding wastes at a federal repository in the year 2005 as a function of age . . . . . . . . . . .

Table 4.33 Ingestion toxicity $\left(\mathrm{m}^{3}\right.$ water) of accumulated fission product isotopes in the cladding wastes at a federal repository in the year 2005 as a function of age . . . 
Table 4.34 Projected annual accumulation of intermediate-level transuranium waste at a federal repository . . . . . 58

Table 4.35 Grams of accumulated heavy elements in intermediateradiation-level TRU wastes at a federal repository in the year 2005 as a function of age . . . . . . . 59

Table 4.36 Curies of accumulated heavy isotopes in intermediateradiation-level TRU wastes at a federal repository in the year 2005 as a function of age . . . . . . . 60

Table 4.37 Watts of accumulated heavy isotopes in intermediateradiation-level TRU wastes at a federal repository in the year 2005 as a function of age ....... 61

Table 4.38 Ingestion toxicity $\left(\mathrm{m}^{3}\right.$ water) of accumulated heavy isotopes in intermediate-radiation-level TRU wastes at a federal repository in the year 2005 as a function of age ................ 62

Table 4.39 'Grams of accumulated fission product elements in. intermediate-radiation-level TRU wastes at a fedëral repository in the year 2005 as a function of age . . . 63

Table 4.40 Curies of accumulated fission product isntopes in intermediate-radiation-level TRU wastes at a federal repository in the year 2005 as a function of age . . . 64

Table 4.41, Watts of accumulated fission product isotopes in intermediate-radiation-level TRU wastes at a federal repository in the year 2005 as a function of age . . . 65

Table 4.42 Ingestion toxicity $\left(\mathrm{m}^{3}\right.$ water) of accumulated fission product isotopes in intermediate-radiation-level TRU wastes at a federal repository in the year 2005 as a function of age ............... 66

Table 4.43 Projected annual accumulation of low-level plutonium transuranic waste at a federal repository . . . . . 68

Table 4.44 Projected annual accumulations of low-level U-233 wastes at a federal repository . . . . . . . 70

Table 4.45 Grams of accumulated heavy elements in low-level plutonium TRU wastes at a federal repository in the year 2005 as a function of age . . . . . . . . . 71

Table 4.46 Curies of accumulated heavy isotopes in low-level plutonium TRU wastes at a federal remsitory in the year 2005 as a Function of age . . . . . . . . . 72 
Table 4.47 Watts of accumulated heavy isotopes in low-level plutonium TRU wastes at a federal repository in the year 2005 as a function of age . . . . . . . 73

Table 4.48 Ingestion toxicity $\left(\mathrm{m}^{3}\right.$ water) of accumulated heavy isotopes in low-level plutonium TRU wastes at a federal repository in the year 2005 as a function of age . .

Table 4.49 Grams of accumulated heavy elements in low-level U-233 wastes at a federal repository in the year 2005 as a funçtion of age . . . . . . . . . .

Table 4.50 Curies of accumulated heavy isotopes in low-level U-233 wastes at a federal repository in the year 2005 as a function of age ............

Table 4.51 Watts of accumulated heavy isotopes in low-level U-233 wastes at a federal repository in the year 2005 as a function of age ............

Table 4.52 Ingestion toxicity ( ${ }^{3}$ water) of accumulated heavy isotopes in low-level U-233 wastes at a federal repository in the year 2005 as a function of age . .

Table 4.53 Projected annual accumulation of noble-gas

fission products... . . . . . . . . . . .

Table 4.54 Projected annual accumulation of fission product iodine . . . . . . . . . . . . . . . . . . .

Table 4.55 Projected annual accumulation (Ci) of carbon-14 from reactor fuels . . . . . . . . . . . . .

Table 4.56 Projected annual accumulation of tritiated water from reactors ...............

Table 4.57 Projected annual accumulation of fission product tritium .....................

Table 4.58 Projected annual accumulation of low-level nontransuranic waste from reactors . . . . . . .

Table 4.59

Projected annual accumulation of low-level nontransuranic waste at surface burial grounds . . .

Table 4.60 Projected annual accumulation of uranium ore tailings .......................

Table 4.61 Projected annual accumulation of thorium ore tailings . . . . . . . . . . . . . . . . . 
UPDATED PROJECTIONS OF RADIOACTIVE WASTES TO BE

GENERATED BY THE U.S. NUCLEAR POWER INDUSTRY

C. W. Kee, A. G. Croff, J. O. Blomeke

ABSTRACT

\begin{abstract}
Eleven types of radioactive wastes to be generated within the fuel cycle operations of the U.S. nuclear power industry are defined, and projections are presented of their annual generation rates, shipping requirements, and accumulated characteristics over the remainder of this century. The power reactor complex is assumed to consist of uranium- and plutonium-fueled LWRs, HTGRs, and LMFBRs, and the installed nuclear electric capacity of the U.S. is taken as 68.1, 252, and $510 \mathrm{GW}$ at the ends of calendar years 1980, 1990, and 2000, respectively.
\end{abstract}

\title{
1. INTRODUCTION
}

Projections of the industrial radioactive wastes from the nuclear fuel cycle are useful in the planning and design of methods and facilities that will be needed for their future management, and for the assessment nf potential cnvironmental effects. until recently, many design and planning studies were based on the waste projections published in Projections of Radioactive Wastes to be Generated by the U.S. Nuclear Power Industry, ORNL-TM-3965 (February 19\%4).1 However, as a result of changes in the projected growth of installed nuclear electrlc capacity and in the types and characteristics of the reactors, of changes in fuel cycle stratcgies and waste inanagement technology, and of increased interest in waste types not included in previous projections (viz., ${ }^{14} \mathrm{C}$ ), more current and comprehensive projections are required. The present study was made to fulfill this need. 
In this study, the "Mid Case" forecast ${ }^{2}$ of nuclear power growth in the United States as devëloped by ERDA's Office of Assistant Administrator for Planning and Analysis (APA) was used (Table 1.1). This case assumes a kilowatt-hour growth-rate of $5.8 \%$ per year through 1985 , and $4.75 \%$ per year through 2000. Additional APA assumptions are as follows:

1. Fast breeder reactors will be commercially introduced in 1995 and will achieve 6\% of the installed nuclear capacity by 2000 .

2. HTGRs will increase to $2 \%$ of the non-breeder market. during the 1990's.

3. LWRs will constitute the remainder of the market with two-thirds as PWRs and one-third as BWRs.

4. Fuel reprocessing begins in 1981.

5. Plutonium recycle begins in 1983 with $25 \%$ of the plutonium inventory, increases to $50 \%$ of the inventory in 1984, and to $75 \%$ in 1985 . It is recycled without constraint thereafter.

6. Nuclear reactor capacity factors are:

lst year of operation $-40 \%$

2nd and 3rd year of operation - 65\%

4 th through 15 th years of operation - $70 \%$

16th through 40 th years of operation - declines 2 percentage points per year to $40 \%$.

Six types of nuclear reactors are considered in these projections: uranium-enriched PWR (PWR-U); plutonium-enriched PWR (PWR-Pu); uraniumenriched BWR (BWR-U); plutonium-enriched BWR (BWR-PU); LMFBR; and HTGR:

To estimate the future magnitude of waste management operations, fuel cycle process flowsheets were constructed, assuming present and near-future technology, and eleven types of radioactive wastes were defined on the basis of their packaging, shipping, and probable disposal requirements. Finally, annual rates of generation; annual numbers of shipments, and accumulations of each type of waste through the end of 
Table 1.1. Projected nuclear operating capazity, GW(e)

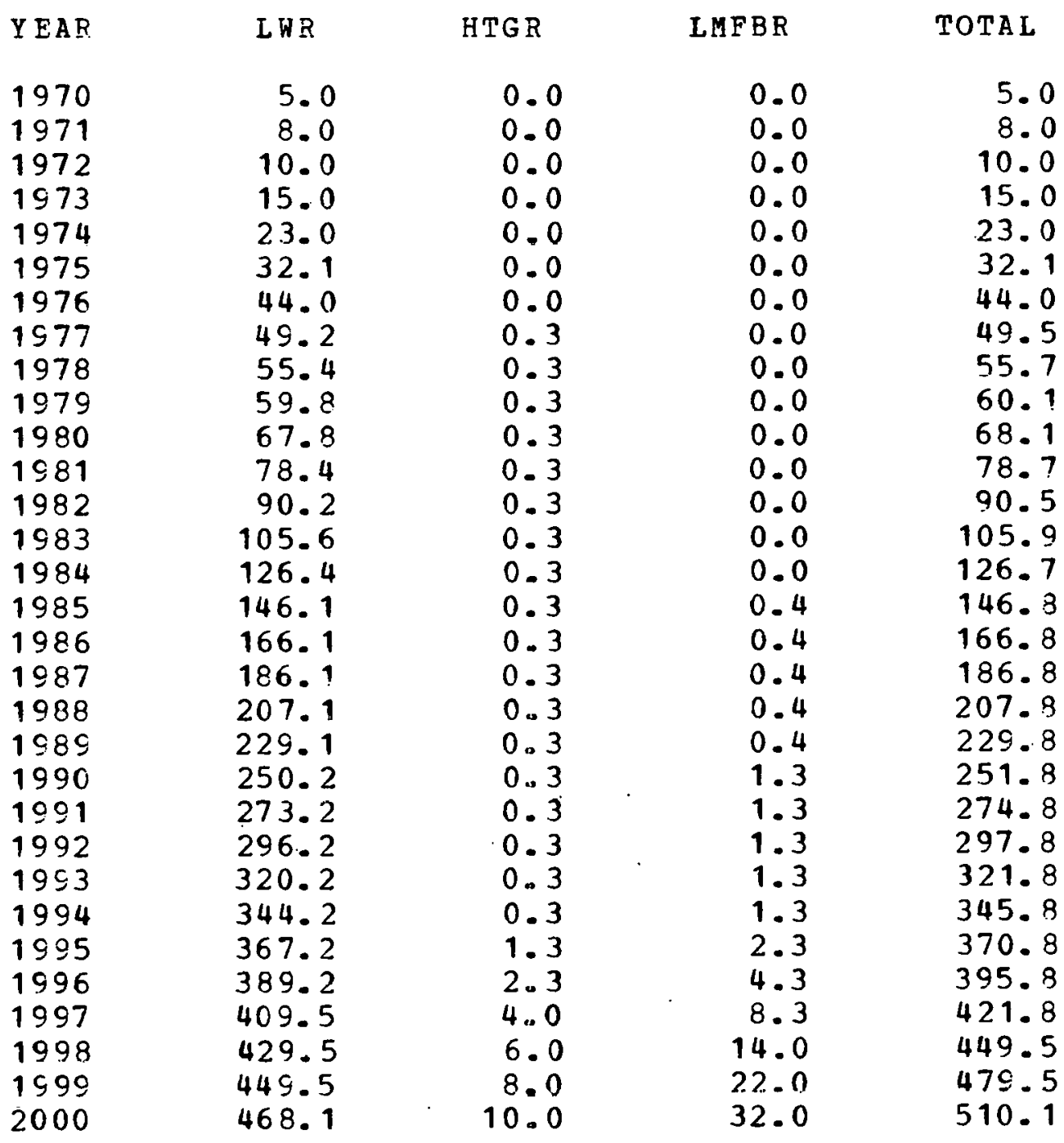


this century were calculated. A summary of these projections for the year 2000 is presented in Table 1.2.

As a matter of possible interest, the characteristics of the principal fuel cycle wastes on the basis of the production of $1000 \mathrm{MW}-$ years of electricity by LWRs (50\% PWRs and 50\% BWRs) fueled with approximately $1 / 3$ mixed (U-Pu) oxide elements and $2 / 3$ enriched $\mathrm{UO}_{2}$ elements are given in Table 1.3.

\section{ASSUMED NUCLEAR REACTOR CHARACTERISTICS}

Tables 2.1 and 2.2 present the major reactor characteristics which were assumed in these waste projections. These two tables represent middle-of-reactor-life mass flows only. Beginning- and end-of-reactor-life mass flows are accounted for in the projections of nuclear fuel management that are presented in section 3, but are not taken into account here.

\subsection{Light Water Reactors}

The physical and operational (e.g., specific power) characteristics of the reference light water reactors were taken from PWR and BWR standard safety analysis reports. ${ }^{3,4}$ Isotopic buildup and depletion in the four types of LWRs are based on charye and discharge data given in Ref. 5 for the following isotopes: ${ }^{234} \mathrm{U},{ }^{235} \mathrm{U},{ }^{236} \mathrm{U},{ }^{238} \mathrm{U},{ }^{237} \mathrm{~Np}$, ${ }^{238} \mathrm{Pu},{ }^{239} \mathrm{Pu},{ }^{240} \mathrm{Pu},{ }^{241} \mathrm{Pu},{ }^{242} \mathrm{Pu},{ }^{241} \mathrm{Am},{ }^{242} \mathrm{Am},{ }^{243} \mathrm{Am},{ }^{242} \mathrm{Cm},{ }^{243} \mathrm{Cm}$, and ${ }^{244} \mathrm{Cm}$. This represents a substantial improvement over the LWRs used in previous waste projections, which were based on charge and discharge data for only ${ }^{235} \mathrm{U},{ }^{238} \mathrm{U},{ }^{238} \mathrm{Pu},{ }^{239} \mathrm{Pu},{ }^{240} \mathrm{Pu},{ }^{241} \mathrm{Pu}$, and $242 \mathrm{Pu}$.

An additional improvement over previous waste projections is the explicit inclusion of both uranium- and plutonium-enriched BWRs. Pertinent reactor and fuel cycle characteristics were obtained from Refs. 4 and 5 .

The fractions of the plutonium-recycle LWRs (PWR-Pu and BWR-Pu) that are assumed to be plutonium-enriched are based on equating the second-cycle fissile plutonium discharged to the third-cycle fissile 
Table 1.2. Fuel cycle wastes projected for the year 2000

\begin{tabular}{|c|c|c|c|c|c|c|}
\hline \multirow[b]{2}{*}{ Category of wastes } & \multicolumn{2}{|c|}{ Annual generation } & \multirow[b]{2}{*}{$\begin{array}{c}\text { Annual } \\
\text { shipments }\end{array}$} & \multicolumn{3}{|c|}{ Total accumulated at repository } \\
\hline & $\begin{array}{l}\text { Volume } \\
\left(10^{3} \mathrm{~m}^{3}\right)\end{array}$ & $\begin{array}{l}\text { Activity } \\
\text { (MCi) }\end{array}$ & & $\begin{array}{l}\text { Volume } \\
\left(10^{3} \mathrm{~m}^{3}\right)\end{array}$ & $\begin{array}{l}\text { Activity } \\
\text { (MCi) }\end{array}$ & $\begin{array}{l}\text { Metric tons } \\
\text { of actinides }\end{array}$ \\
\hline & & & & & & \\
\hline High-level solidified & 0.88 & 24,000 & $200^{\mathrm{a}, \mathrm{b}}$ & 2.2 & 5,900 & 150 \\
\hline \multicolumn{7}{|l|}{ Transuranium wastes } \\
\hline Cladding hulls & 0.78 & 86 & $450^{b, c}$ & 4.5 & 260 & $31^{\circ}$ \\
\hline Int.-level solid & 3.9 & 1.3 & $1,350^{c, d}$ & 20 & 5.8 & 0.16 \\
\hline Low-level solid & 12 & 10 & $250^{c, b}$ & 58 & 37 & 4.5 \\
\hline Non-TRU wastes & & & & & & \\
\hline Noble gases & $0.029^{f}$ & 73 & $110^{\mathrm{b}, \mathrm{e}}$ & $0.29^{f}$ & 550 & - \\
\hline Iodine & 0.013 & 0.00034 & $49^{e, d}$ & 0.13 & 0.0033 & - \\
\hline Carbon-14 & - & 0.005 & - & - & 0.058 & - \\
\hline LWR tritium (water) & 65 & 0.3 & $4,100^{e, d}$ & 730 & 2.4 & - \\
\hline FP tritijm (solidified) & 0.094 & 4.9 & $350^{e, d}$ & 0.91 & 36 & - \\
\hline Low-level solid & 260 & 0.92 & $19,000^{e, d}$ & 2,700 & 3.2 & - \\
\hline Ore tailings & 20,000 & 0.24 & - & 300,000 & 3.4 & 48,000 \\
\hline
\end{tabular}

${ }^{a}$ Shipped 10 years after generation.

${ }_{\text {Rail shipments are assumed. }}$

${ }^{C}$ shipped 5 years after generation.

$\mathrm{d}_{\text {Truck shipments are assumed. }}$

eshipped 1 year after generation.

$\mathrm{f}_{\text {Pressurized at }} 2200$ psi. 
Table 1.3. Fuel cycle wastes from the production of 1000 MW-years of electricity ${ }^{a}$

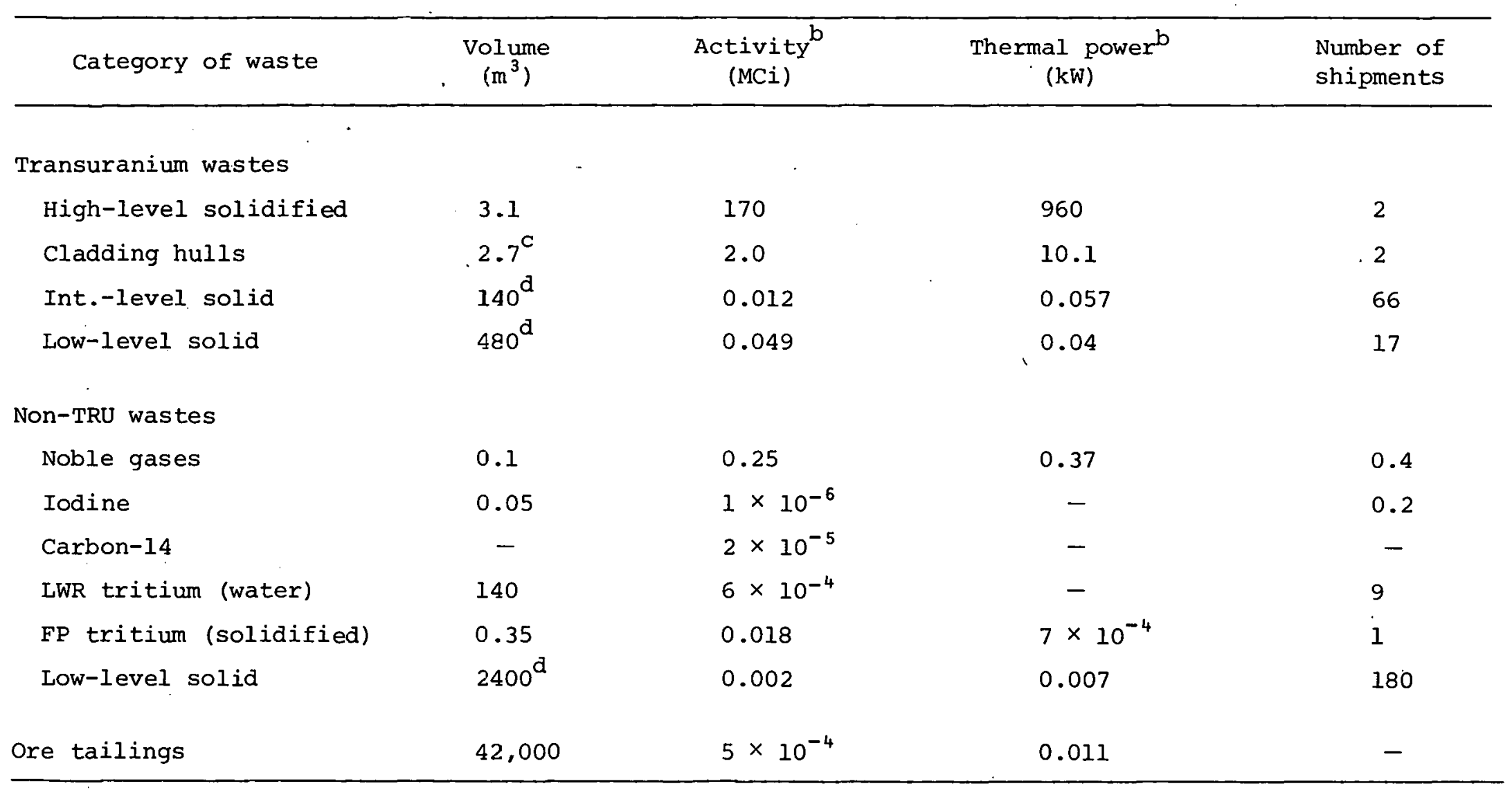

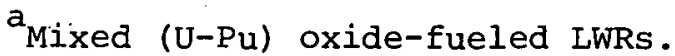

${ }^{b}$ Activity and thermal power at time of waste generation.

${ }^{C}$ Compacted to $70 \%$ of theoretical density.

$\mathrm{d}_{\text {Uncompacted volume. }}$ 
Table 2.1 Assumed characteristics of light water reactors

\begin{tabular}{|c|c|c|c|c|c|c|c|c|}
\hline & \multirow[b]{2}{*}{ PNR-U } & \multicolumn{3}{|c|}{ PWR-Pu } & \multirow[b]{2}{*}{ BWR-U } & \multicolumn{3}{|c|}{$\mathrm{BWR}-\mathrm{Pu}$} \\
\hline & & $\begin{array}{c}\mathrm{E} \\
\text { fraction }\end{array}$ & $\begin{array}{c}\mathrm{Pu} \\
\text { fraction }\end{array}$ & Total & & $\begin{array}{c}\mathrm{U} \\
\text { fraction }\end{array}$ & $\begin{array}{c}\mathrm{Pu} \\
\text { fraction }\end{array}$ & Total \\
\hline Electric হower, MW(e) & 1250 & $8 \pm 1$ & 439 & 1250 & 1250 & 776 & 474 & 1250 \\
\hline Thermal power, MW $(t)$ & 3800 & 2465 & 1335 & 3800 & 3800 & 2359 & 1441 & 3800 \\
\hline $\begin{array}{l}\text { Avg. specific power }{ }^{a}, \\
M W(t) / \text { ton heavy metal }\end{array}$ & $3^{7} .5$ & 37.5 & 37.5 & 37.5 & 25.9 & 25.9 & 25.9 & 25.9 \\
\hline $\begin{array}{l}\text { Avg. fuel burnup, } \\
\text { Mha/ton heavy metal }\end{array}$ & $3 \equiv, 000$ & 33,050 & 33,000 & 33,000 & 27,500 & 27,500 & 27,500 & 27,500 \\
\hline $\begin{array}{l}\text { Irradiaticn duration, } \\
\text { full-power days }\end{array}$ & $8 \varepsilon 0$ & 880 & 880 & 880 & 1062 & 1062 & 1062 & 1062 \\
\hline \multicolumn{9}{|l|}{ Stea:̈y-state charge, $\mathrm{kg}$} \\
\hline $235_{U}$ & 1031 & 701 & 81 & 782 & 943 & 585 & 95 & 680 \\
\hline Total U & 33.778 & 21,915 & 11,323 & 33,238 & 36,680 & 22.771 & 13,364 & 36,135 \\
\hline Fissile $\geq \mathrm{u}^{\mathrm{b}}$ & 0 & 0 & 345 & 345 & 0 & 0 & 338 & 338 \\
\hline Total $\mathrm{Pu}^{\mathrm{C}}$ & 0 & 0 & 539 & 539 & 0 & 0 & 546 & 546 \\
\hline Total heavy metal & 33.778 & 21,915 & 11,862 & 33.777 & 36,680 & 22,771 & 13,910 & 36,681 \\
\hline \multicolumn{9}{|c|}{ Steaćy-s tate discharge, $\mathrm{kg}$} \\
\hline $235_{\mathrm{U}}$ & $27 \bar{z}$ & 176 & 37 & 213 & 244 & 151 & 46 & 197 \\
\hline Total U & 32,261 & 20,931 & 11,031 & 31,962 & 35,278 & 21,901 & 13,077 & 34,978 \\
\hline Fissile Fu & 222 & 144 & 201 & 345 & 220 & 137 & 201 & 338 \\
\hline Tot: $1 \mathrm{Pu}^{\mathrm{C}}$ & 316 & 205 & 378 & 583 & 315 & 196 & 389 & 585 \\
\hline Total (U + Pu) & 32,577 & 21,136 & 11,409 & 32,545 & 35,593 & 22,097 & 13,466 & 35,563 \\
\hline
\end{tabular}

a Based upon full fower and Euel charged.

${ }^{b_{239}} \mathrm{Pu}+{ }^{241_{\mathrm{Pu}}}$.

${ }^{c_{238}} \mathrm{pu}+{ }^{239} \mathrm{Pu}+{ }^{240} \mathrm{Pu}+{ }^{24}{ }_{-\mathrm{Pu}}+{ }^{242} \mathrm{Pu}$. 
Table 2.2 Assumed characteristics of the HTGR and the A.I. Follow-On LMFBR

\begin{tabular}{|c|c|c|c|c|c|c|c|c|}
\hline \multirow[b]{2}{*}{ - } & \multicolumn{4}{|c|}{ H'TGR } & \multicolumn{4}{|c|}{ A.I. Follow-On LMFBR } \\
\hline & $\mathrm{Th}-{ }^{233} \mathrm{U}$ & $\begin{array}{c}\text { Fresh } \\
235_{\mathrm{U}} \\
\text { makeuy }\end{array}$ & $\begin{array}{l}\text { Recycled } \\
235_{U} \\
\text { makeup }\end{array}$ & Total & ccre & $\begin{array}{c}\text { Axial } \\
\text { blanket }\end{array}$ & $\begin{array}{r}\text { Radial } \\
\text { blanket }\end{array}$ & Total \\
\hline Electric power, MW(e) & 807 & 409 & 34 & 1250 & 1147 & 39 & 64 & 1250 \\
\hline Thermal power, Mw $(t)$ & 2087 & 1058 & 87 & 3232 & 2748 & 94 & 153 & 2995 \\
\hline $\begin{array}{l}\text { Avg. specific power }{ }^{a} \text {, } \\
M W(t) / \text { ton heavy metal }\end{array}$ & 55.1 & 584 & 203 & 80.5 & 115 & 5.71 & 7.90 & 50.25 \\
\hline $\begin{array}{l}\text { Avg. fuel burnup, } \\
\text { Mwd/ton heavy metal }\end{array}$ & 64,210 & 682,260 & 238,040 & 93,972 & 67,100 & 3330 & 13,270 & 37,137 \\
\hline $\begin{array}{l}\text { Irradiation duration, } \\
\text { full-power days }\end{array}$ & 1167 & 1157 & 1167 & 1167 & 583 & 583 & 1680 & - \\
\hline \multicolumn{9}{|l|}{ Steady-state charge, $\mathrm{kg}$} \\
\hline Th & 9087 & 0 & 0 & 9087 & 0 & 0 & 0 & 0 \\
\hline${ }^{233} \mathrm{v}$ & 236 & 0 & 0 & 234 & 0 & 0 & 0 & 0 \\
\hline $235_{U}$ & 32 & 419 & 23 & 474 & 20 & 16 & 6.7 & 43 \\
\hline Total U. & 386 & 453 & 107 & 946 & 9850 & 8213 & 3352 & 21,415 \\
\hline Fissile $\mathrm{Pu}^{\mathrm{b}}$ & 0 & 0 & 0 & 0 & 14.61 & 0 & 0 & 1461 \\
\hline Total $\mathrm{Pu}^{\mathrm{c}}$ & 0 & 0 & 0 & 0 & 2077 & 0 & 0 & 2077 \\
\hline Total heavy metal & 9473 & 453 & 107 & 10,033 & 11,927 & 8213 & 3352 & 23,492 \\
\hline \multicolumn{9}{|c|}{ Steady-state.discharge, $\mathrm{kg}$} \\
\hline Th & 8427 & 0 & 0 & 8427 & 0 & 0 & 0 & 0 \\
\hline${ }^{233} \mathrm{U}$ & 211 & 0 & 0 & 211 & 0 & 0 & 0 & 0 \\
\hline $235 \mathrm{v}$ & 40 & 23 & 1.4 & 64 & 11 & 14 & 4.4 & 29 \\
\hline Total U & 384 & 108 & 65 & 557 & 9037 & 8049 & 3165 & 20,251 \\
\hline Fissile $\mathrm{Pu}^{\mathrm{b}}$ & 0 & 1.6 & 1.6 & 3.1 & 1344 & 129 & 132 & 1605 \\
\hline Total Puc & 0 & 7.0 & 7.3 & 14 & 2017 & 132 & 139 & 2288 \\
\hline Total $(\mathrm{Th}+\mathrm{U}+\mathrm{Pu})$ & 8811 & 115 & 72 & 8998 & 11,054 & 8181 & 3304 & 22.539 \\
\hline
\end{tabular}

Based on full power and fuel charged.

$\mathrm{b}_{239} \mathrm{Pu}+{ }^{241} \mathrm{Pu}$.

$\mathrm{c}_{238} \mathrm{Pu}+{ }^{239} \mathrm{Pu}+{ }^{240} \mathrm{Pu}+{ }^{241} \mathrm{Pu}+{ }^{242} \mathrm{Pu}$. 
plutonium charged (i.e., equilibrium fissile plutonium recycle is assumed). Then, given a required fissile plutonium enrichment (2.91 wt $\%$ fissile plutonium in PWRs and 2.43 wt $\%$ fissile plutonium in BWRs), the percentage of the fuel which is plutonium-enriched was calculated to be $35.1 \%$ for the PWR-Pu and $37.9 \%$ for the BWR-Pu. This procedure results in the total plutonium discharged in the second cycle being slightly greater than the total plutonium charged in the third cycle for both the PWR-Pu and BWR-Pu. This effect is unavoidable since the second and third plutonium recycles are not equilibrium recycles, and the total plutonium (including the fissile fraction) discharge is increasing with each cycle.

\subsection{High-Temperature Gas-Cooled Reactor}

The reference HTGR is based upon design studies of an 1160-MW(e) HTGR that uses annual refueling (at $80 \%$ capacity factor) of approximately one-fourth of the core. ${ }^{6}, 7$ Recycle of ${ }^{235} \mathrm{U}$ is assumed to begin in the third reload. The ${ }^{235} \mathrm{U}$ from the fissile makeup particles is assumed to be reprocessed separately from the ${ }^{233} \mathrm{U}$-thorium particles and recycled one time before discard. This recycle of partially burned ${ }^{235} \mathrm{U}$ particles is also assumed to begin in the third reload.

\subsection{A. I. Follow-On LMFBR}

The assumed characteristics of the Atomics International (A. I.) Follow-On LMFBR are taken from normalized data developed by Argonne National Laboratory. ${ }^{8}$

\section{PROJECTIONS OF NUCLLEAR FUEL MANAGEMENT}

The nuclear generating capacity and mass flow information presented in the previous sections were employed in the KWIKPLAN code ${ }^{9}$ to derive a schedule of requirements for the nuclear fuel cycle. The assumed processing steps, lag times, and losses were the same as those employed by the Fuel Recycle Task Force ${ }^{10}$ with exceptions that the LWR, HTGR, and LMFBR fuels were assumed to be reprocesscd after post-irradiation decay times of 160, 250, and 90 days, respectively. The data used by 
KWIKPLAN were schedules of installed nuclear power capacity, on-stream fuel reprocessing plant capacity, and the fuel mass flows for the reference reactors. The installed capacities and reprocessing rates were taken from the APA study, ${ }^{2}$ while the fuel mass-flow data were taken from the reactor models presented in Tables 2.1 and 2.2.

The reactor models were described in KWIKPLAN as a year-by-year schedule of reactor operating capacity factors, and the fuel charged and discharged from each reactor during its 40-year operating life. The code then calculated the uranium and thorium mining requirements, the utilization of fissile materials, and reactor fuel fabrication and reprocessing loads. The increased fabrication and reprocessing requirements that result from the initial startup and final shutdown of each reactor were taken into account.

Table 3.1 presents a summary of the computed annual requirements for mining of uranium and thorium and the production and utilization of fissile plutonium, ${ }^{23}{ }^{3} \mathrm{U}$, and highly enriched uranium. The requirements for mining of uranium are based upon a tails assay in uranium enrichment of $0.3 \%{ }^{235} \mathrm{U}$ and makes allowance for the recycle for reenrichment of uranium that contains greater than $0.4 \%{ }^{235} \mathrm{U}$. The annual "production" of fissile materials is defined as the annual quality that is placed in a stockpile following reprocessing. The quantities in the columns labeled "used" are the annual quantities withdrawn from this stockpile. Also included with the ${ }^{233} \mathrm{U}$ are relatively small quantities of ${ }^{235} \mathrm{U}$ that are produced in HTGR fertile particles.

Table 3.2 presents the derived annual loads of the several types of fuel fabrication plants expressed in terms of metric tons of total heavy metal. The fabrication load for plutonium recycle tó light water reactors includes only those portions of the fuel assemblies that are fueled with mixed oxide (about 35 to $38 \%$ of the total in the core). 
Table 3.1 Metric tons of 0 . Th, and Pu consumed, produced, and stockpiled annuaily

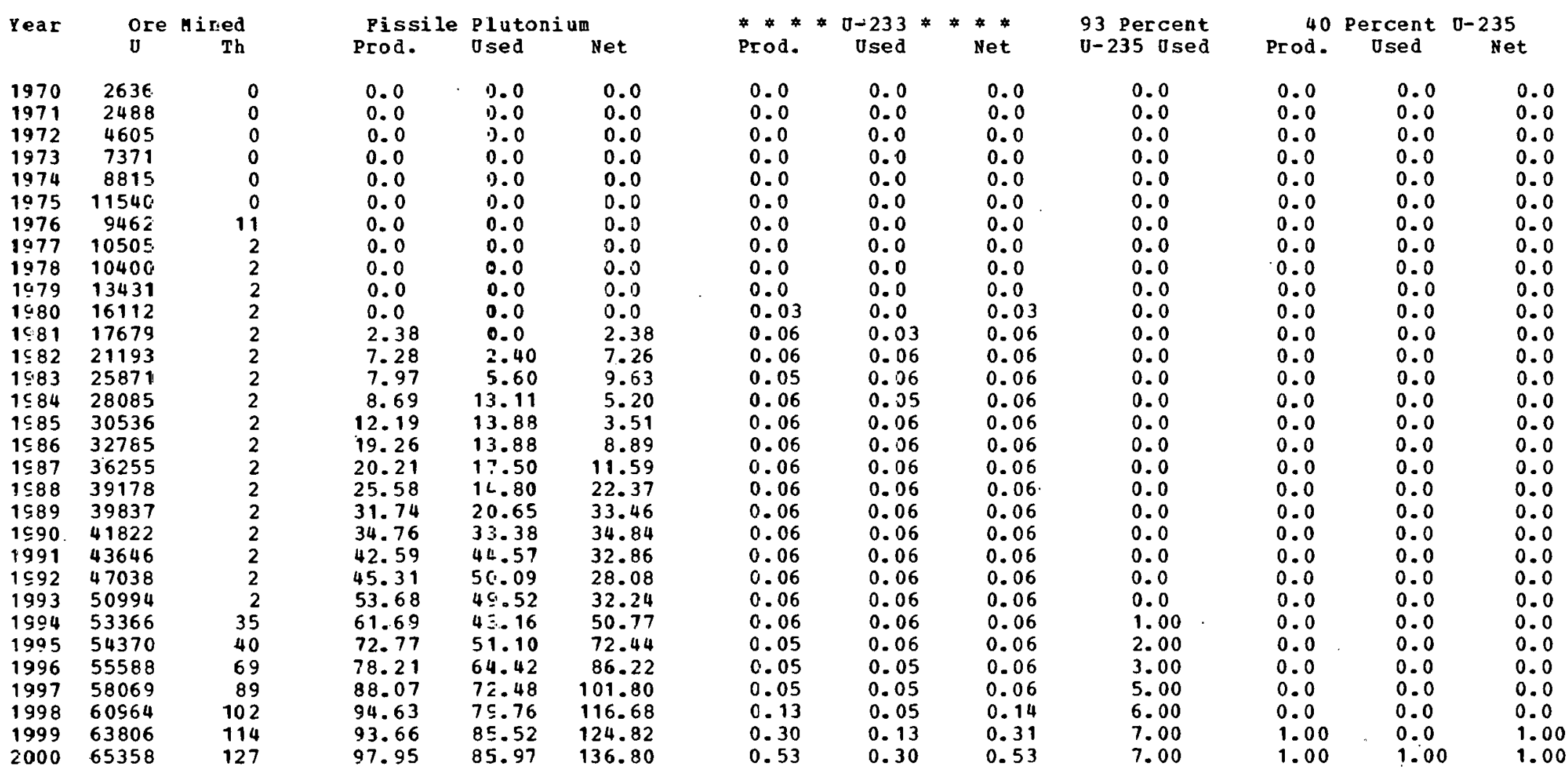


Table 3.2. Metric tons of fuel fabricated annually

$\begin{array}{lrrrrrrr}\text { Year } & \text { FWR-U } & \text { PWR-Pu } & \text { BWR-U } & \text { B WR-Pu } & \text { HTGR } & \text { L MF BR } & \text { Total } \\ 1970 & 139 & 0 & 231 & 0 & 0 & 0 & 370 \\ 1971 & 156 & 0 & 281 & 0 & 0 & 0 & 437 \\ 1972 & 220 & 0 & 387 & 0 & 0 & 0 & 607 \\ 1973 & 357 & 0 & 668 & 0 & 0 & 0 & 1026 \\ 1974 & 587 & 0 & 770 & 0 & 0 & 0 & 1357 \\ 1975 & 1014 & 0 & 620 & 0 & 0 & 0 & 1635 \\ 1976 & 1120 & 0 & 565 & 0 & 6 & 0 & 1691 \\ 1977 & 1109 & 0 & 527 & 0 & 7 & 0 & 1642 \\ 1978 & 1209 & 0 & 541 & 0 & 2 & 0 & 1752 \\ 1979 & 1244 & 0 & 805 & 0 & 2 & 0 & 2052 \\ 1980 & 1436 & 0 & 1113 & 0 & 2 & 0 & 2552 \\ 1981 & 1785 & 0 & 1109 & 0 & 2 & 0 & 2896 \\ 1982 & 2125 & 56 & 1137 & 32 & 2 & 0 & 3352 \\ 1983 & 2464 & 132 & 1490 & 73 & 2 & 0 & 4161 \\ 1984 & 2548 & 293 & 1881 & 160 & 2 & 18 & 4902 \\ 1985 & 2812 & 315 & 2066 & 184 & 2 & 5 & 5383 \\ 1986 & 3200 & 312 & 2117 & 188 & 2 & 5 & 5824 \\ 1987 & 3458 & 394 & 2275 & 237 & 2 & 5 & 6372 \\ 1988 & 3895 & 332 & 2542 & 200 & 2 & 5 & 6977 \\ 1989 & L 119 & 447 & 2666 & 269 & 2 & 27 & 7529 \\ 1990 & 4192 & 732 & 2711 & 439 & 2 & 33 & 8108 \\ 1991 & \text { L306 } & 1000 & 2790 & 600 & 2 & 17 & 8716 \\ 1992 & 4552 & 1127 & 2936 & 676 & 2 & 19 & 9310 \\ 1993 & 4941 & 1113 & 3171 & 668 & 2 & 18 & 9913 \\ 1994 & 5437 & 945 & 3457 & 567 & 19 & 42 & 10467 \\ 1995 & 5594 & 1076 & 3544 & 645 & 39 & 96 & 10995 \\ 1996 & 5632 & 1292 & 3550 & 775 & 58 & 187 & 11493 \\ 1997 & 5838 & 1361 & 3643 & 816 & 84 & 298 & 12039 \\ 1998 & 6081 & 1334 & 3826 & 799 & 102 & 486 & 12628 \\ 1999 & 6402 & 1263 & 3998 & 756 & 115 & 670 & 13203 \\ 2000 & 6724 & 1028 & 4274 & 616 & 129 & 887 & 13658\end{array}$


Table 3.3 gives the projected fuel reprocessing load, or the quantity of spent fuel that will be discharged each year. The projected schedule of fuel actually reprocessed is given in Table 3.4. The indicated backlog of 1365 tons of heavy metal as spent fuel in inventory at the end of 1975 (Table 3.4) was taken from a recent survey. ${ }^{11}$ This table includes the estimated low-exposure fuels from reactor startup cycles as well as scrap that is recycled from fuel fabrication. According to this schedule, the backlog of unreprocessed spent fuel will not be worked off until near the end of this century.

In considering the delay in reprocessing, it was assumed that fuels from demonstration HTGRs and LMFBRs would be reprocessed by ERDA pilot plant facilities. Since mixed oxide LWR fuels contain greater amounts of fissile plutonium, they were assumed to be reprocessed preferentially to $\mathrm{UO}_{2}$ fuels. The $\mathrm{UO}_{2}$ fuels were reprocessed from the backlog on a first-in, first-out basis since this would allow reprocessing plants to work with older feedstocks during the earlier years of operation.

Table 3.5 is essentially a table of megawatt-days of exposure represented by the fuel reprocessed in a given year since it presents the equivalent tons of fuel having the rated steady-state exposures. These data were used to develop estimates of the radiation properties of aggregates of spent fuel and wastes based upon calculated properties of the spent fuel at the rated steady-state exposures as determined with the ORIGEN ${ }^{12}$ code.

Since spent fuel may be stored for an extended time before reprocessing occurs, calculations were made of the number of LWR fuel assemblies to be discharged and accumulated (if reprocessing was not available) through the year 2000 (Table 3.6). This calculation assumes $461.4 \mathrm{~kg}$ of heavy metal and $183.35 \mathrm{~kg}$ of heavy metal per PWR and BWR assembly, respectively $\cdot{ }^{3}, 4$ Again, the number of assemblies accumulated by the end of 1975 were taken from ERDA-76-25. ${ }^{11}$ 
Table 3.3. Annual reprocessing load (Metric Tons)

\begin{tabular}{|c|c|c|c|c|}
\hline Y EAR & $\mathrm{LWR}$ & HTGR & LMFBR & TOTAL \\
\hline 1970 & 59 & 0 & 0 & 50 \\
\hline 1971 & 95 & 0 & 0 & 95 \\
\hline 1972 & 156 & 0 & 0 & 156 \\
\hline 1973 & 217 & 0 & 0 & 217 \\
\hline 1974 & 301 & 0 & 0 & 301 \\
\hline 1975 & 459. & 0 & 0 & 459 \\
\hline 1976 & 663 & 0 & 0 & 663 \\
\hline 1977 & 897 & 0 & 0 & 897 \\
\hline 1978 & 1088 & 0 & 0 & 1088 \\
\hline 1979 & 1228 & 2.2 & 0 & 1230 \\
\hline 1980 & 1356 & 2.2 & 0 & 1358 \\
\hline 1981 & 1507 & 2.3 & 0 & 1509 \\
\hline 1982 & 1733 & 2.3 & 0 & 1735 \\
\hline 1983 & 1991 & 2.1 & 0 & 1993 \\
\hline 1984 & 2296 & 2.1 & 0 & 2298 \\
\hline 1985 & 2717 & 2.1 & 0 & 2719 \\
\hline 1986 & 3197 & 2.1 & 4.8 & 3203 \\
\hline 1987 & 3675 & 2.1 & 4.7 & 3681 \\
\hline 1988 & 4141 & 2.1 & 5.1 & 4148 \\
\hline 1989 & 4620 & $2 . .1$ & 5.1 & 4628 \\
\hline 1990 & 5124 & 2.1 & 5.1 & 5131 \\
\hline 1991 & 5620 & 2.1 & 16.5 & 5639 \\
\hline 1992 & 6121 & 2.1 & 16.2 & 6140 \\
\hline 1993 & 6640 & 2.1 & 17.1 & 6659 \\
\hline 1994 & 7166 & 2.0 & 17.1 & 7185 \\
\hline 1905 & 7700 & 2.0 & 17.1 & 7719 \\
\hline 1996 & 8220 & 1.9 & 26.6 & 8249 \\
\hline 1997 & 8712 & 8.6 & 48.5 & 8769 \\
\hline 1998 & 9167 & 14.9 & 93.1 & 9275 \\
\hline 1999 & .9595 & 26.6 & 157.1 & 9779 \\
\hline 2000 & 9993 & 40.0 & 256.6 & 10290 \\
\hline
\end{tabular}


Table 3.4. Fuel reprocessed (Metric Tons)

$\begin{array}{lrrrrr}\text { YEAR } & \text { L NR } & \text { HTGR } & \text { LMFBR } & \text { TOTAL } & \text { BACKLOG } \\ 1975 & 0 & 0 & 0 & 0 & 1365 \\ 1976 & 0 & 0 & 0 & 0 & 2029 \\ 1977 & 0 & 0 & 0 & 0 & 2926 \\ 1978 & 0 & 0 & 0 & 0 & 4014 \\ 1979 & 0 & 2 & 0 & 2 & 5241 \\ 1980 & 0 & 2 & 0 & 2 & 6597 \\ 1981 & 500 & 2 & 0 & 502 & 7603 \\ 1982 & 1500 & 2 & 0 & 1502 & 7836 \\ 1983 & 1500 & 2 & 0 & 1502 & 8327 \\ 1984 & 1500 & 2 & 0 & 1502 & 9123 \\ 1985 & 2000 & 2 & 0 & 2002 & 9840 \\ 1986 & 3000 & 2 & 5 & 3007 & 10036 \\ 1987 & 3000 & 2 & 5 & 3007 & 10711 \\ 1088 & 3500 & 2 & 5 & 3507 & 11352 \\ 1989 & 4500 & 2 & 5 & 4507 & 11472 \\ 1990 & 5000 & 2 & 5 & 5007 & 11597 \\ 1991 & 6000 & 2 & 17 & 6019 & 11217 \\ 1992 & 6500 & 2 & 16 & 6518 & 10838 \\ 1993 & 7500 & 2 & 17 & 7519 & 9978 \\ 1994 & 8000 & 2 & 17 & 8019 & 9143 \\ 1995 & 9100 & 2 & 17 & 9119 & 7743 \\ 1996 & 9600 & 2 & 27 & 9628 & 6364 \\ 1997 & 11000 & 9 & 49 & 11057 & 4076 \\ 1998 & 12000 & 15 & 93 & 12108 & 1242 \\ 1999 & 10838 & 27 & 157 & 11021 & 0 \\ 2000 & 9903 & 40 & 257 & 10290 & 0\end{array}$


Table 3.5. Metric tons of fuel reprocessed annualiy at rated exposure

$\begin{array}{lrrrrrr}\text { Year } & \text { PWR-U } & \text { PUR-Pu } & \text { BWR-U } & \text { BWR-Pu } & \text { HTGR } & \text { IMFBR } \\ 1979 & 0 & 0 & 0 & 0 & 1 & 0 \\ 1980 & 0 & 0 & 0 & 0 & 1 & 0 \\ 1981 & 182 & 0 & 166 & 0 & 2 & 0 \\ 1982 & 494 & 0 & 574 & 0 & 2 & 0 \\ 1993 & 628 & 0 & 543 & 0 & 2 & 0 \\ 1984 & 772 & 0 & 533 & 0 & 2 & 0 \\ 1985 & 1162 & 0 & 675 & 0 & 2 & 0 \\ 1986 & 1704 & 53 & 929 & 31 & 2 & 3 \\ 1987 & 1602 & 126 & 865 & 70 & 2 & 3 \\ 1988 & 1757 & 280 & 923 & 154 & 2 & 4 \\ 1939 & 2286 & 301 & 1191 & 177 & 2 & 5 \\ 1990 & 2551 & 298 & 1369 & 181 & 2 & 5 \\ 1991 & 3058 & 377 & 1685 & 229 & 2 & 12 \\ 1992 & 3436 & 318 & 1917 & 193 & 2 & 13 \\ 1993 & 3950 & 427 & 2211 & 259 & 2 & 15 \\ 1994 & 4015 & 699 & 2257 & 423 & 2 & 18 \\ 1995 & 4420 & 956 & 2484 & 578 & 2 & 18 \\ 1996 & 4598 & 1076 & 2582 & 651 & 2 & 23 \\ 1997 & 5462 & 1063 & 3081 & 643 & 4 & 37 \\ 1998 & 6264 & 903 & 3562 & 546 & 8 & 67 \\ 1999 & 5490 & 1028 & 3139 & 622 & 15 & 115 \\ 2000 & 4800 & 1235 & 2753 & 746 & 25 & 191\end{array}$


Table 3.6. Number of $L W R$ spent fuel assemblies discharged

\begin{tabular}{|c|c|c|c|c|c|c|}
\hline Year & $\begin{array}{c}\text { Annual } \\
\text { BWF }\end{array}$ & $\begin{array}{r}\text { number } \\
\text { PWR }\end{array}$ & $\begin{array}{r}\text { discharged } \\
\text { Total }\end{array}$ & Total & $\begin{array}{r}\text { number } \\
\text { PWR }\end{array}$ & disch \\
\hline & & & & & & \\
\hline 1975 & 1494 & 401 & 1896 & 4404 & 1321 & 5725 \\
\hline 1976 & 1814 & 555 & 2370 & 6218 & 1877 & 8095 \\
\hline 1977 & 2124 & 979 & 3104 & 8342 & 2856 & 11198 \\
\hline 1978 & 2435 & 1390 & 3825 & 10777 & 4247 & 15023 \\
\hline 1979 & 2524 & 1657 & 4181 & 13301 & 5904 & 19205 \\
\hline 1080 & 2596 & 1906 & 4502 & 15896 & 7810 & 23707 \\
\hline 1991 & 2933 & 2100 & 5033 & 18830 & 9910 & 28740 \\
\hline 1032 & 3548 & 2346 & 5894 & 22377 & 12256 & 34633 \\
\hline 1983 & 4024 & 2717 & 6741 & 26401 & 14972 & 41374 \\
\hline 1984 & 4445 & 3210 & 7654 & 30846 & 18182 & 49028 \\
\hline 1985 & 5231 & 3809 & 9040 & 36077 & 21991 & 58069 \\
\hline 1986 & 6391 & 4388 & 10779 & 42467 & 26379 & 68847 \\
\hline 1987 & 7520 & 4976 & 12496 & 49987 & 31355 & 81343 \\
\hline 1938 & 8453 & 5616 & 14069 & 58441 & 36971 & 95412 \\
\hline 1989 & 9422 & 6270 & 15692 & 67863 & 43241 & 111104 \\
\hline 1990 & 10445 & 6955 & 17400 & 78308 & 50196 & 128504 \\
\hline 1991 & 11436 & 7637 & 19072 & 89744 & 57833 & 147576 \\
\hline 1992. & 12441 & 8323 & 20764 & 102185 & 66155 & 168340 \\
\hline 1993 & 13498 & 9026 & 22524 & 115683 & 75181 & 190864 \\
\hline $19 \subseteq 4$ & 14575 & 9739 & 24313 & 130258 & 84920 & 215178 \\
\hline 1995 & 15669 & 10462 & 26131 & 145927 & 95382 & 241308 \\
\hline 1996 & 16730 & 11168 & 27897 & 162656 & 106549 & 269206 \\
\hline 1997 & 17729 & 11837 & 29566 & 180386 & 118396 & 298772 \\
\hline 1098 & 18656 & 12454 & $3110 \mathrm{c}$ & 199041 & 130840 & 329881 \\
\hline 1999 & 19519 & 13040 & 32559 & 218560 & 143880 & 362440 \\
\hline 2000 & 20361 & 13567 & 33928 & 238921 & 157447 & 396368 \\
\hline
\end{tabular}


4. ORIGIN, NATURE, AND PROJECTIONS OF FUEL CYCLE WASTES

There are many important aspects bearing on waste management that are in evolutionary stages of development. While much research and development work has been done, there remains a shortage of commercial plant operating experience upon which estimates of future performance can be reliably based. This is especially true of those operations that will generate most of the wastes: fuel preparation, fabrication, and reprocessing. We have nevertheless attempted in this section to derive definitions of "typical" wastes and estimate their generation rates by drawing on available operating experience at both ERDA and commercial facilities, and from the results of research and development work, much of which is still in progress.

The characteristics of radioactive wastes from the nuclear fuel cycle, the factors that influence their generation, and the bases used for projecting future quantities were discussed in ORNL/TM-3965. ${ }^{1}$ This information is summarized below with our new projections. For comparative purposes, the principal assumptions used for the waste projections of ORNL/TM-3965 and those used for this study are listed in Table 4.1 .

\subsection{High-Level Wastes}

High-level wastes are defined in federal regulations ${ }^{13}$ as "those aqueous wastes resulting from the operation of the first cycle solvent extraction system, or equivalent, in a facility for reprocessing irradiated reactor fuels." These wastes contain virtually all of the nonvolatile fission products, several tenths of onc percent of the uranium and plutonium originaliy in the spent fuels, and all the other actinides formed by transmutation of the uranium and plutonium in the reactors. They can be generally characterized by their very intense, penetrating radiation and their high heat-generation rates. Regulations call for these wastes to be solidified within 5 years after they are generated and for the resultant stable solids to be shipped to a federal repository within 10 years after the liquids are generated. ${ }^{13}$ 
Table 4.1. Comparison of principal assumptions used for waste projections

\begin{tabular}{|c|c|c|}
\hline Categor of waste & $\begin{array}{l}\text { ORNL/TM-3965 } \\
\quad(\operatorname{Ref} .1)\end{array}$ & This report \\
\hline High-level solidified waste & 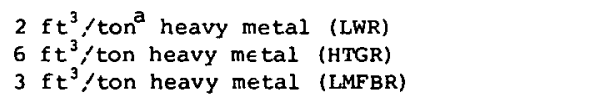 & $\begin{array}{lll}3 & \mathrm{ft}^{3} / \text { ton heavy metal (LWR) } \\
6 \mathrm{ft}^{3} / \text { ton heavy metal (HTGR) } \\
3 \mathrm{ft}^{3} / \text { ton heavy metal (LMFBR) }\end{array}$ \\
\hline Cladding hulls (compacted) & $\begin{array}{l}2.3 \mathrm{f}=3 / \text { ton heavy metal (LWR) } \\
8.7 \mathrm{f}=3 / \text { ton heavy metal (LMFBR) }\end{array}$ & $\begin{array}{l}2.3 \mathrm{ft}^{3} / \text { ton heavy metal (PWR) } \\
2.9 \mathrm{ft}^{3} / \text { ton heavy metal (BWR) } \\
8.7 \mathrm{ft}^{3} / \text { ton heavy metal (LMFBR) }\end{array}$ \\
\hline Intermediata-level solid TRU & $10,000 \mathrm{ft}^{3} /$ ton $\mathrm{Pu}$ (reprocessing) & $10,000 \mathrm{ft}^{3} /$ ton $\mathrm{Pu}$ (reprocessing) \\
\hline Lọ-level solid TRU & $\begin{array}{l}10,000 \mathrm{ft}^{3} / \text { ton Pu (preparation) } \\
20,000 \mathrm{ft}^{3} / \text { ton Pu (fabrication) } \\
4,000 \mathrm{ft}^{3} / \text { ton Pu (reprocessing) }\end{array}$ & $\begin{array}{l}10,000 \mathrm{ft}^{3} / \text { ton Pu (preparation) } \\
20,000 \mathrm{ft}^{3} / \text { ton Pu (fabrication) } \\
4,000 \mathrm{ft}^{3} / \text { ton Pu (reprocessing) }\end{array}$ \\
\hline Noble yases $(K r+x e)$ & $693 \mathrm{~g}$-atoms/cylinder & $693 \mathrm{~g}$-atoms/cylinder \\
\hline Iodine & Storage as $\mathrm{KI}, 587 \mathrm{~g}$-atoms $/ \mathrm{ft}^{3}$ & Storage in concrete, $40.4 \mathrm{~g}$-atoms $/ \mathrm{ft}^{3}$ \\
\hline Carbon-14 & - & $\begin{array}{l}25 \mathrm{ppm} \text { nitrogen in oxide fuels } \\
30 \mathrm{ppm} \text { nitrogen in } 3 T G R \text { graphite }\end{array}$ \\
\hline LWR tritiated water & $\begin{array}{l}20,000 \mathrm{gal} / \text { year per GW }(e) \\
\text { installed capacity }\end{array}$ & $\begin{array}{l}36,600 \mathrm{gal} / \text { year per GW (e) } \\
\text { installed capacity }\end{array}$ \\
\hline FP tritium & Fixed as $\mathrm{Ca}(\mathrm{OH})_{2}, \sim 1500 \mathrm{Ci} / \mathrm{ft}^{3}$ & Fixed as $\mathrm{Ca}(\mathrm{OH})_{2}, \sim 1500 \mathrm{Ci} / \mathrm{ft}^{3}$ \\
\hline Low-level solid non-TRU & $\begin{array}{l}40,000 \mathrm{ft}^{3} / \text { ton Pu (preparation) } \\
80,000 \mathrm{ft}^{3} / \text { ton Pu (fabrication) } \\
16,000 \mathrm{ft}^{3} / \text { ton Pu (reprocessing) } \\
4,000 \mathrm{ft}^{3} / \text { year per GW (e), LWRs, HTGRs } \\
2,000 \mathrm{ft}^{3} / \text { year per GW(e), LMFBRs } \\
\text { Prep. r fab., reproc. , wastes: } 0.001 \mathrm{Ci} / \mathrm{ft}^{3} \\
\text { Reactor wastes: } 1 \mathrm{Ci} / \mathrm{ft}^{3}\end{array}$ & 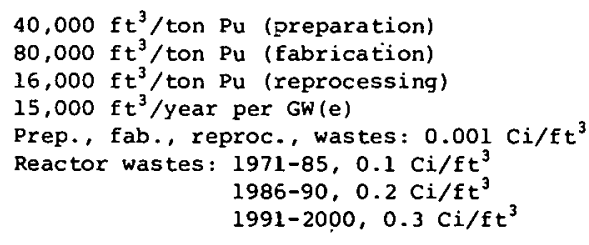 \\
\hline Ore Tailings & $\begin{array}{l}\text { Mining requirements based on } \mathrm{U} \\
\text { enrichment tails } 0.28 \mathrm{~s}^{2} \mathrm{U} \\
\text { Mill tailings: } 0.16 \mathrm{yd}^{3} / 1 \mathrm{~b} \mathrm{\textrm {U } _ { 3 }} \mathrm{O}_{\mathrm{B}} \\
\text { or } \mathrm{ThO}_{2}\end{array}$ & 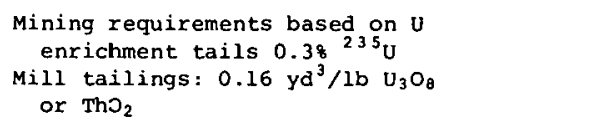 \\
\hline
\end{tabular}

an this table, "ton" is usæd to mean metric ton. 


\subsubsection{Fuel Reprocessing ${ }^{1}$}

In the reprocessing of LWR fuels, a chop-leach head-end method was used, followed by a standard Purex solvent extraction procedure for separating the uranium and plutonium and subsequently purifying the uranium, followed by an amine extraction for the final purification of plutonium. Soluble poisons were not used for criticality control during dissolution.

The flowsheet for reprocessing HTGR fuel was based on methods being developed at Gulf General Atomic, Allied Chemical Corporation at Idaho Falls, Idaho, and ORNL. ${ }^{14}$ The fuel block is crushed prior to burning the graphite in a fluidized bed. The burning step is carried out in two parts: (1) an exothermic burning mode with heat evolution, and (2) an endothermic mode with heat addition. The residual fuel particles are separated from each other by screening, and the $235_{\mathrm{U}}$ (recycle) particles constitute a solid waste which is not processed further. The fertile $\mathrm{ThO}_{2}-{ }^{23}{ }^{3} \mathrm{UO}_{2}$ (recycle) particles are dissolved and, after clarification, are purified by a modified Thorex solventextraction process. ${ }^{15}$ The ${ }^{235} \mathrm{U}$ (makeup) particles are crushed in a roll qrinder to fracture the silicon carbide coating, and the residual carbon is burned off in an exothermic burner. After dissolution and clarification, the ${ }^{235} \mathrm{U}$ is recovered using a TBP-25 solvent-extraction process flowsheet. ${ }^{16}$

The flowsheet for reprocessing LMFBR mixed core and blanket fuel was also based on developmental work still in progress. Following a mechanical disassembly of the fuel element and separation of hardware that has only induced activity associated with it, the fuel tubes are sheared and heated to about $400^{\circ} \mathrm{C}$ in an oxygen atmosphere for removal of volatile fission products (voloxidation). ${ }^{17}$ The oxide fuel is then leached from the stainless steel cladding with nitric acid, and the uranium and plutonium are then recovered by a modified Purex solvent extraction process. The primary flowsheet relied on concentration and geometry for criticality control, and an alternative flowsheet used boron as a soluble poison for this purpose. 


\subsubsection{Waste Volumes}

Estimated characteristics of the residues obtained from evaporating and heating aqueous wastes to $900^{\circ} \mathrm{C}$ are given in Ref. 1 . In addition to the solids obtained from solidifying the individual waste streams derived from the flowsheets, solidification of several combinations of these streams is considered. Based on the flowsheets described above, the combined volumes of solidified reprocessing wastes (excluding cladding) should lie in the neighborhood of 1 to $2 \mathrm{ft}^{3}$ per ton of LWR fuel, 4 to $6 \mathrm{ft}^{3}$ per ton of HTGR fuel, and 1 to $3 \mathrm{ft}^{3}$ per ton of LMFBR fuel. Recent development work indicates that the volume of solidified high-level waste from LWR fuel reprocessing will probably be between 1 and $3 \mathrm{ft}^{3}$ per ton of fuel. ${ }^{18}$ Although waste management economics will tend to force the final volumes toward smaller values, additional wastes not considered here arising from maloperations, equipment decontamination, etc., will tend to increase them. Similarly, possible future requirements for higher-quality waste products (less leachable in water, more radiation-resistant) will also probably result in higher volumes of waste per ton of fuel processed. It is for these reasons that we believe the upper-limit volumes of $3 \mathrm{ft}^{2}, 6 \mathrm{ft}^{3}$, and $3 \mathrm{ft}^{3}$ of solidified high-level waste per ton of fuel processed are the most realistic and, consequently, should be used for planning and design studies at this time.

\subsubsection{Radiation Characteristics of High-Level Wastes}

Appendix A presents the calculated radioactivity and thermal power in high-level wastes generated by reprocessing one metric ton of each of the six types of spent reactor fuels. The tables summarize the curies of fission-product and actinide isotopes as a function of time (years) following reprocessing. In these tables, the columns labeled "discharge" refer to the times when the wastes are first generated at the reprocessing plant and correspond to post-irradiation decay times of 160, 250, and 90 days for the spent LWR, HTGR, and LMFBR fuels, respectively. All of the tritium and noble-gas fission products, and $99.9 \%$ of the iodine and bromine fission products are excluded from the tables on the basis that these 
elements will be separated from the high-level wastes during the headend reprocessing steps. Also, the levels of actinides in the wastes are based on the assumption that $0.5 \%$ of the uranium and plutonium in the LWR and LMFBR fuels, $0.5 \%$ of the thorium and uranium in the HTGR fuel, and $100 \%$ of all other actinides in the fuels at the time of reprocessing report to the high-level waste.

\subsubsection{High-Level Waste Projections}

The activity of fission product and actinide isotopes, the thermal power from radioactive decay, ard the volumes (assuming immediate solidification) of the high-level wastes generated annually by LWRs, HTGRs, and LMFBRs through the year 2000 are presented in Tables 4.2 through 4.4 .

The volume, mass, radioactivity, and toxicity of all high-level wastes accumulated annually through the year 2000 are given in Table 4.5. The activity and thermal power added annually in this table are those at the end of the year. This table includes wastes in storage at reprocessing plants as well as any which have been shipped to federal repositories. The toxicity of the mixture is determined by summing the amount of water or air required to dilute each individual isotope to the maximum level specified in the Radiation Concentration Guide $^{19}$ as acceptable for unrestricted use.

Although solidification of industrial high-level wastes has not as yet been practiced, indications are that these materials may be packaged in steel canisters typically $1 \mathrm{ft}$ in diam by $10 \mathrm{ft}$ long and then shipped to a repository after having first been stored at the reprocessing plants for 10 years. Studies of conceptually designed shipping systems for these wastes ${ }^{20,21}$ show that as many as 12 such canisters (each containing $6.28 \mathrm{ft}^{3}$ of 10-year-old solidified waste) could be shipped by rail in iron- or lead-shielded casks weighing in the neighborhood of 100 tons. Based on these assumptions, the activity, thermal power, and volumc of the 10-year-old wastes that will be shipped annually are given in Tables 4.6, 4.7, and 4.8, respectively. The number of solidified waste containers to be 
Table 4.2. Radioactivity (MCi) in high-level wastes generated annually

$\begin{array}{lrrrr}\text { YEAR } & \text { LWR } & \text { HTGR } & \text { LMFBR } & \text { TOTAL } \\ 1979 & 0 & 4 & 0 & 4 \\ 1980 & 0 & 8 & 0 & 8 \\ 1981 & 1535 & 12 & 0 & 1546 \\ 1982 & 4709 & 14 & 0 & 4723 \\ 1983 & 5167 & 13 & 0 & 5180 \\ 1984 & 5753 & 13 & 0 & 5766 \\ 1985 & 8105 & 13 & 0 & 8118 \\ 1986 & 12031 & 13 & 26 & 12070 \\ 1987 & 11849 & 13 & 32 & 11994 \\ 1988 & 13957 & 13 & 41 & 14012 \\ 1989 & 17686 & 13 & 50 & 17750 \\ 1990 & 19644 & 13 & 50 & 19707 \\ 1991 & 23898 & 13 & 113 & 24024 \\ 1902 & 26118 & 13 & 126 & 26258 \\ 1993 & 30548 & 13 & 148 & 30709 \\ 1994 & 33184 & 12 & 169 & 33365 \\ 1995 & 37997 & 12 & 169 & 38178 \\ 1996 & 40161 & 12 & 221 & 40393 \\ 1997 & 46075 & 25 & 353 & 46454 \\ 1998 & 50471 & 47 & 642 & 51160 \\ 1990 & 46178 & 92 & 1103 & 47373 \\ 2000 & 43054 & 155 & 1840 & 45049\end{array}$


Table 4.3. Thermal power ( $k$ w) in high-level wastes generated annually

$\begin{array}{lrrrr}\text { YEAR } & \text { LWR } & \text { HTGR } & \text { LMFBR } & \text { TOTAL } \\ 1979 & 0 & 20 & 0 & 20 \\ 1980 & 0 & 37 & 0 & 37 \\ 1981 & 7219 & 54 & 0 & 7273 \\ 1982 & 22151 & 66 & 0 & 22216 \\ 1983 & 24305 & 60 & 0 & 24364 \\ 1984 & 27062 & 60 & 0 & 27122 \\ 1985 & 38126 & 60 & 0 & 38186 \\ 1986 & 57579 & 60 & 113 & 57751 \\ 1987 & 58032 & 60 & 138 & 58229 \\ 1988 & 70729 & 60 & 170 & 70968 \\ 1989 & 88780 & 60 & 217 & 89057 \\ 1990 & 97993 & 60 & 217 & 98270 \\ 1991 & 119484 & 60 & 488 & 120032 \\ 1992 & 128821 & 60 & 547 & 129428 \\ 1993 & 151712 & 60 & 643 & 152414 \\ 1994 & 169205 & 57 & 730 & 169992 \\ 1995 & 196651 & 57 & 730 & 197438 \\ 1996 & 209092 & 54 & 955 & 210101 \\ 1997 & 236666 & 114 & 1531 & 238311 \\ 1998 & 254344 & 217 & 2783 & 257343 \\ 1999 & 236490 & 422 & 4781 & 241692 \\ 2000 & 225660 & 710 & 7973 & 234342\end{array}$


Table 4.4. Cubic meters of solidified high-level wastes generated annually

$\begin{array}{lrrrr}\text { YEAR } & \text { LWR } & \text { HTGR } & \text { LMFBR } & \text { TOTAL } \\ 1979 & 0 & 0.4 & 0 & 0.4 \\ 1980 & 0 & 0.4 & 0 & 0.4 \\ 1981 & 42 & 0.4 & 0 & 43 \\ 1982 & 127 & 0.4 & 0 & 128 \\ 1983 & 127 & 0.4 & 0 & 128 \\ 1984 & 127 & 0.4 & 0 & 128 \\ 1985 & 170 & 0.4 & 0 & 170 \\ 1986 & 255 & 0.4 & 0.4 & 256 \\ 1987 & 255 & 0.4 & 0.4 & 256 \\ 1988 & 297 & 0.4 & 0.4 & 298 \\ 1989 & 382 & 0.4 & 0.4 & 333 \\ 1990 & 425 & 0.4 & 0.4 & 425 \\ 1991 & 509 & 0.4 & 1.4 & 511 \\ 1992 & 552 & 0.4 & 1.4 & 554 \\ 1993 & 637 & 0.4 & 1.5 & 639 \\ 1994 & 679 & 0.3 & 1.5 & 681 \\ 1995 & 773 & 0.3 & 1.5 & 774 \\ 1996 & 815 & 0.3 & 2.3 & 818 \\ 1997 & 934 & 1.5 & 4.1 & 940 \\ 1998 & 1019 & 2.5 & 7.9 & 1029 \\ 1999 & 920 & 4.5 & 13.3 & 938 \\ 2000 & 848 & 6.8 & 21.8 & 277\end{array}$


Table 4.5. Projected annual accumulations of solidified high-level wastes without shipment

\begin{tabular}{|c|c|c|c|c|}
\hline ear & $\begin{array}{l}\text { volume } \\
\text { (Thousands } \\
\text { of } \mathrm{m}^{3} \text { ) }\end{array}$ & $\begin{array}{l}\text { Annual } \\
\text { Actinide } \\
\text { Hass } \\
\text { (MT) }\end{array}$ & $\begin{array}{l}\text { Radio- } \\
\text { activity } \\
\text { (MCi) }\end{array}$ & $\begin{array}{c}\text { Thermal } \\
\text { Pover } \\
\text { (UW) }\end{array}$ \\
\hline $\begin{array}{l}1979 \\
1980 \\
1981 \\
1982 \\
1983 \\
1984 \\
1985 \\
1986 \\
1987 \\
1988 \\
1989 \\
1990 \\
1991 \\
1992 \\
1993 \\
1994 \\
1995 \\
1996 \\
1997 \\
1998 \\
1999 \\
2000\end{array}$ & $\begin{array}{l}0.00 \\
0.00 \\
0.04 \\
0.13 \\
0.13 \\
0.13 \\
0.17 \\
0.26 \\
0.26 \\
0.30 \\
0.38 \\
0.43 \\
0.51 \\
0.55 \\
0.64 \\
0.68 \\
0.77 \\
0.82 \\
0.94 \\
1.03 \\
0.94 \\
0.88\end{array}$ & $\begin{array}{l}0.0 \\
0.0 \\
2.8 \\
8.4 \\
8.5 \\
8.5 \\
11.4 \\
17.3 \\
17.5 \\
20.8 \\
26.6 \\
29.4 \\
35.4 \\
38.1 \\
44.1 \\
47.8 \\
54.9 \\
58.2 \\
66.3 \\
71.8 \\
66.2 \\
62.8\end{array}$ & $\begin{array}{r}3 \\
5 \\
788 \\
2359 \\
2644 \\
2991 \\
4260 \\
6361 \\
6276 \\
7410 \\
9383 \\
10397 \\
12653 \\
13817 \\
16158 \\
17557 \\
20095 \\
21263 \\
24441 \\
26892 \\
24894 \\
23665\end{array}$ & $\begin{array}{r}0 \\
0 \\
4 \\
12 \\
13 \\
15 \\
21 \\
31 \\
31 \\
38 \\
48 \\
53 \\
64 \\
69 \\
82 \\
90 \\
104 \\
111 \\
126 \\
137 \\
128 \\
123\end{array}$ \\
\hline
\end{tabular}

* * * * Accumulation Through End of
Volume Actinide Radio- Theral
(Thousands Mass activity Power
of $\left.{ }^{3}\right)$
(MT)

0.00

0.04

0.17

0.30

0.43

0.60

0. 85

1. 11

1.41

1.79

2. 22

2.73

3. 28
3.92

3.92
4.60

5. 38

6.19

7. 13

8. 16

9. 98

\begin{abstract}
0. 0
\end{abstract}

0.1
2.9

2.9
11.3

19.8

28.4

39.8

57.0

74.5

95.4

122.0

151.4

186.8

224.8

268.9

316.7

371.6

429.8

496.1

567.9

634.

696.9

3
7$$
\begin{array}{r}
792 \\
2757
\end{array}
$$

$$
4086
$$

5275

7338

10687

12575

15104

18771

22115

26674

30802

36045

40964

46997

52285

59365

66663

69818

71809

9.98
9.98
9.98
9.98
9.98
9.98
9.98
9.98
9.98

696.9

696. 9

696.9

3042.3

95.6

27.2

696.9

696. 9

696.9

696.9

13.8

8. 7

3. 9

2. 0

696.9

696.9

1.5
0.8

0
0
4
13
20
25
35
51
60
73
91
107
129
148
172
197
227
253
287
320
334
343

343

$\begin{array}{rrrr}11.9 & & 5.6 \mathrm{E} & 20 \\ 1.7 & & 3.1 \mathrm{E} & 20 \\ 0.7 & & 1.3 \mathrm{E} & 20 \\ 0.3 & & 6.8 \mathrm{E} & 19 \\ 0.2 & & 4.5 \mathrm{E} & 19 \\ 0.1 & & 2.1 \mathrm{E} & 19 \\ 0.0 & & 4.1 \mathrm{E} & 18 \\ 0.0 & & 2.6 \mathrm{E} & 18 \\ 0.0 & & 2.7 \mathrm{E} & 18\end{array}$

2. $7 \mathrm{E} 18$ Hazard, Cubic

Meters at RCG

Air

Water

$\begin{array}{llll}1.8 \mathrm{E} & 16 & 8.5 \mathrm{E} & 11\end{array}$

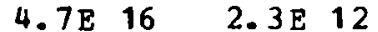

$\begin{array}{llll}5.6 \mathrm{E} & 18 & 1.2 \mathrm{E} & 14\end{array}$

2. $1 \mathrm{E} 19 \quad 4.5 \mathrm{E} 14$

$\begin{array}{llll}3.6 \mathrm{E} & 19 & 7.8 \mathrm{E} & 14\end{array}$

$\begin{array}{llll}5.2 \mathrm{E} & 19 & 1.1 \mathrm{E} & 15\end{array}$

$\begin{array}{llll}7.5 \mathrm{E} & 19 & 1.6 \mathrm{E} & 15\end{array}$

1.3E 20 2. $2 \mathrm{E} 15$

1. 9 E $20 \quad 3.0 \mathrm{E} \quad 15$

3. 1E 20 3. $8 \mathrm{E} \quad 15$

4. $3 \mathrm{E} 20 \quad 4.7 \mathrm{E} 15$

5.6E $20 \quad 5.8 \mathrm{E} \quad 15$

7. 2 E 20 7.0E 15

$8.6 \mathrm{E} \quad 20 \quad 8.4 \mathrm{E} \quad 15$

1. 0 E 21 1. $0 \mathrm{E} 16$

1. $3 \mathrm{E} 21$ 1. $2 \mathrm{E} 16$

1. $7 \mathrm{E} 21$ 1. $3 \mathrm{E} 16$

$2.0 \mathrm{E} \quad 21 \quad 1.5 \mathrm{E} 16$

2. 4E $21 \quad 1.8 \mathrm{E} \quad 16$

$2.7 \mathrm{E} \quad 21 \quad 2.0 \mathrm{E} \quad 16$

$\begin{array}{llll}3.0 \mathrm{E} & 21 & 2.2 \mathrm{E} & 16\end{array}$

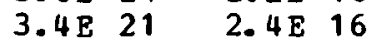

300000
1000000

1. $8 \mathrm{E} \quad 15$

2. $5 E \quad 13$

4. $9 \mathrm{E} 12$

$1.8 E \quad 12$

1. $1 \mathrm{E} 12$

4.7E 11

3. $7 \mathrm{E} 11$

4. $6 \mathrm{E} 11$

2. $7 \mathrm{E} 11$ 
Table 4.6. Radioactivity (MCi) in 10-year-old high-level wastes shipped annually

$\begin{array}{lrrrr}\text { YEAR } & \text { LWR } & \text { HTGR } & \text { LMFBR } & \text { TOTAL } \\ 1989 & 0 & 1 & 0 & 1 \\ 1990 & 0 & 1 & 0 & 1 \\ 1991 & 109 & 2 & 0 & 111 \\ 1992 & 336 & 2 & 0 & 338 \\ 1993 & 368 & 2 & 0 & 370 \\ 1994 & 410 & 2 & 0 & 412 \\ 1995 & 578 & 2 & 0 & 580 \\ 1996 & 859 & 2 & 1 & 862 \\ 1997 & 848 & 2 & 1 & 851 \\ 1998 & 1003 & 2 & 1 & 1006 \\ 1999 & 1270 & 2 & 2 & 1273 \\ 2000 & 1409 & 2 & 2 & 1413 \\ 2001 & 1715 & 2 & 4 & 1721 \\ 2002 & 1871 & 2 & 4 & 1877 \\ 2003 & 2190 & 2 & 5 & 2197 \\ 2004 & 2386 & 2 & 6 & 2394 \\ 2005 & 2737 & 2 & 6 & 2744 \\ 2006 & 2895 & 2 & 7 & 2904 \\ 2007 & 3316 & 4 & 12 & 3332 \\ 2008 & 3624 & 8 & 21 & 3653 \\ 2009 & 3322 & 15 & 36 & 3373 \\ 2010 & 3106 & 25 & 61 & 3191\end{array}$


Table 4.7. Thermal power (kw) in 10-year-old high-level wastes shipped annually

$\begin{array}{lrrrr}\text { YEAR } & \text { LWR } & \text { HTGR } & \text { LMFBR } & \text { TOTAL } \\ 1989 & 0 & 3 & 0 & 3 \\ 1990 & 0 & 5 & 0 & 5 \\ 1901 & 379 & 7 & 0 & 386 \\ 1992 & 1164 & 9 & 0 & 1172 \\ 1993 & 1277 & 8 & 0 & 1285 \\ 1994 & 1422 & 8 & 0 & 1429 \\ 1995 & 2003 & 9 & 0 & 2011 \\ 1996 & 3233 & 8 & 3 & 3243 \\ 1997 & 3534 & 8 & 3 & 3545 \\ 1998 & 4789 & 8 & 4 & 4801 \\ 1999 & 5845 & 8 & 5 & 5858 \\ 2000 & 6331 & 8 & 5 & 6343 \\ 2001 & 7773 & 8 & 11 & 7791 \\ 2002 & 8028 & 8 & 12 & 8048 \\ 2003 & 9666 & 8 & 14 & 9688 \\ 2004 & 11663 & 7 & 16 & 11687 \\ 2005 & 14122 & 7 & 16 & 14146 \\ 2006 & 15254 & 7 & 21 & 15282 \\ 2007 & 16650 & 15 & 34 & 16699 \\ 2008 & 16944 & 28 & 62 & 17034 \\ 2009 & 16502 & 55 & 107 & 16663 \\ 2010 & 16751 & 92 & 178 & 17021\end{array}$


Table 4.8. Cubic meters of 10-year-old high-level wastes shipped annually

\begin{tabular}{|c|c|c|c|c|}
\hline YEAR & $\mathrm{LWR}$ & HTGR & LMFBR & TOTAL \\
\hline 1989 & 0 & 0.4 & 0 & 0.4 \\
\hline 1990 & 0 & 0.4 & 0 & 0.4 \\
\hline $1 \subseteq 91$ & 42 & 0.4 & 0 & 43 \\
\hline 1992 & 127 & 0.4 & 0 & 128 \\
\hline 1093 & 127 & 0.4 & 0 & 128 \\
\hline 1994 & 127 & 0.4 & 0 & 129 \\
\hline $19 \subseteq 5$ & 170 & 0.4 & 0 & 170 \\
\hline 1995 & 2.55 & 0.4 & 0.4 & 256 \\
\hline 1997 & 255 & 0.4 & 0.4 & 2.56 \\
\hline 1998 & 297 & 0.4 & 0.4 & 299 \\
\hline $19 \varsigma 9$ & 382 & 0.4 & 0.4 & 383 \\
\hline 2000 & 425 & 0.4 & 0.4 & 425 \\
\hline 2001 & 509 & 0.4 & 1.4 & 511 \\
\hline 2002 & 552 & 0.4 & 1.4 & 554 \\
\hline 2003 & 637 & 0.4 & 1.5 & 639 \\
\hline 2004 & 679 & 0.3 & 1.5 & 681 \\
\hline 2005 & 773 & 0.3 & 1.5 & 774 \\
\hline 2006 & 815 & 0.3 & 2.3 & 818 \\
\hline 2007 & 934 & 1.5 & 4.1 & 940 \\
\hline 2008 & 1019 & 2.5 & 7.9 & 1029 \\
\hline 2000 & 920 & 4.5 & 13.3 & 038 \\
\hline 2010 & 848 & 6.8 & 21.8 & 877 \\
\hline
\end{tabular}


shipped annually and the number of shipments are presented in Tables 4.9 and 4.10. The annual and cumulative properties (volume, mass, radioactivity, thermal power, and toxicity) of the solidified highlevel wastes accumulated at one or more federal repositories are presented in Table 4.11. The number of annual shipments is also shown.

The accumulated mass, radioactivity, thermal power, and ingestion toxicity of the solidified high-level wastes as a function of time following the year 2010 are summarized in Tables 4.12 through 4.15 for actinides, and in Tables 4.16 through 4.19 for fission products.

\subsection{Fuel Element Structural Materials (Cladding Wastes)}

Fuel element structural materials are comprised of the zircaloy, stainless steel, Inconel, and other miscellaneous materials which comprise the structure of a fuel assembly. These materials remain in the fuel dissolver after the fuel itself has been as completely dissolved as is possible. Although their radioactivity arises mainly from neutroninduced isotopes, the structural materials are similar in some respects to high-level waste in that they may contain up to $0.1 \%$ of the plutonium originally in the spent fuel, need biological shielding equivalent to several inches of lead, and have heat-generation rates of 50 to $100 \mathrm{~W} / \mathrm{ft}^{3}$.

The mass, volume, and assumed exposure of the residual structural materials from one metric ton of PWR, BWR, and LMFBR fuel are summarized in Table 4.20. The percentage exposure reflects the fact that the structural material, which is not in close proximity to the fuel itselt, (e.g., the stainless steel end fittings in LWRs) is exposed to a reduced flux level as compared to the cladding. This results in less activation of these components.

The neutron-induced radioactivity and thermal power of the major nuclides in the irradiated structural materials associated with one metric ton of LWR and LMFBR fuels are given in Appendix $B$ for the five reactor types assumed. In addition to induced activity, the cladding 
Table 4.9. Number of 10-year-old high-level waste canisters shipped annually

$\begin{array}{lrrrr}\text { YEAR } & \text { LWR } & \text { HTGR } & \text { LMFBR } & \text { TOTAL } \\ 1989 & & & 0 & 2 \\ 1990 & 0 & 2 & 0 & 2 \\ 1991 & 239 & 2 & 0 & 241 \\ 1992 & 717 & 2 & 0 & 719 \\ 1993 & 717 & 2 & 0 & 719 \\ 1994 & 717 & 2 & 0 & 719 \\ 1995 & 955 & 2 & 0 & 957 \\ 1996 & 1433 & 2 & 2 & 1437 \\ 1997 & 1433 & 2 & 2 & 1437 \\ 1998 & 1672 & 2 & 2 & 1676 \\ 1999 & 2150 & 2 & 2 & 2154 \\ 2000 & 2389 & 2 & 2 & 2393 \\ 2001 & 2866 & 2 & 8 & 2876 \\ 2002 & 3105 & 2 & 8 & 3115 \\ 2003 & 3583 & 2 & 8 & 3593 \\ 2004 & 3822 & 2 & 8 & 3832 \\ 2005 & 4347 & 2 & 8 & 4357 \\ 2006 & 4586 & 2 & 13 & 4600 \\ 2007 & 5255 & 8 & 23 & 5286 \\ 2008 & 5732 & 14 & 45 & 5791 \\ 2009 & 5177 & 25 & 75 & 5278 \\ 2010 & 4774 & 38 & 123 & 4935\end{array}$


Table 4.10. Number of annual 10-year-old high-level waste shipments

\begin{tabular}{lrrrr} 
YEAR & \multicolumn{1}{c}{ LWR } & \multicolumn{1}{l}{ HTGR } & LMFBR & TOTAL \\
1989 & 0.0 & 0.2 & 0.0 & 0.2 \\
1990 & 0.0 & 0.2 & 0.0 & 0.2 \\
1991 & 19.9 & 0.2 & 0.0 & 20.1 \\
1992 & 59.7 & 0.2 & 0.0 & 59.9 \\
1093 & 50.7 & 0.2 & 0.0 & 59.0 \\
1994 & 59.7 & 0.2 & 0.0 & 59.9 \\
1995 & 79.6 & 0.2 & 0.0 & 79.9 \\
1996 & 119.4 & 0.2 & 0.2 & 119.8 \\
1997 & 119.4 & 0.2 & 0.2 & 119.8 \\
1998 & 139.3 & 0.2 & 0.2 & 139.7 \\
1999 & 179.1 & 0.2 & 0.2 & 179.5 \\
2000 & 199.0 & 0.2 & 0.2 & 199.4 \\
2001 & 238.9 & 0.2 & 0.7 & 239.7 \\
2002 & 258.8 & 0.2 & 0.6 & 259.6 \\
2003 & 298.6 & 0.2 & 0.7 & 299.4 \\
2004 & 318.5 & 0.2 & 0.7 & 319.3 \\
2005 & 362.3 & 0.2 & 0.7 & 363.1 \\
2006 & 382.2 & 0.2 & 1.1 & 383.4 \\
2007 & 437.9 & 0.7 & 1.9 & 440.5 \\
2008 & 477.7 & 1.2 & 3.7 & 482.6 \\
2009 & 431.4 & 2.1 & 6.3 & 439.8 \\
2010 & 397.8 & 3.2 & 10.2 & 411.2
\end{tabular}


Table 4.11. Projected annual accumulation of solidified high-level waste at a federal repository

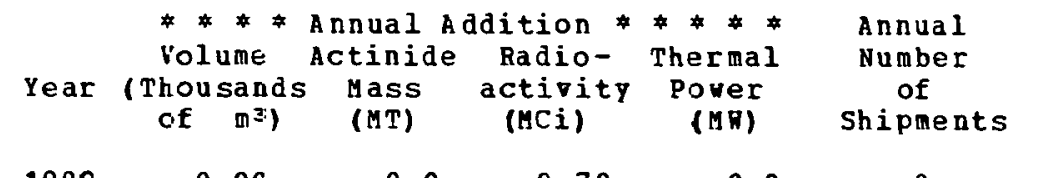

$\begin{array}{rrrrrr}1989 & 0.00 & 0.0 & 0.70 & 0.0 & 0 \\ 1990 & 0.00 & 0.0 & 1.30 & 0.0 & 0 \\ 1991 & 0.04 & 2.8 & 98.58 & 0.3 & 20 \\ 1992 & 0.13 & 8.4 & 295.33 & 1.0 & 60 \\ 1993 & 0.13 & 8.5 & 328.36 & 1.1 & 60 \\ 1994 & 0.13 & 8.5 & 369.35 & 1.3 & 60 \\ 1995 & 0.17 & 11.4 & 523.62 & 1.8 & 80 \\ 1996 & 0.26 & 17.3 & 776.10 & 2.8 & 120 \\ 1997 & 0.26 & 17.5 & 761.04 & 2.8 & 120 \\ 1998 & 0.30 & 20.8 & 889.91 & 3.5 & 140 \\ 1999 & 0.38 & 26.6 & 1129.59 & 4.4 & 180 \\ 2000 & 0.43 & 29.4 & 1254.50 & 4.8 & 199 \\ 2001 & 0.51 & 35.4 & 1524.95 & 5.9 & 240 \\ 2002 & 0.55 & 38.1 & 1571.22 & 6.3 & 260 \\ 2003 & 0.64 & 44.1 & 1950.81 & 7.5 & 299 \\ 2004 & 0.68 & 47.8 & 2105.11 & 8.5 & 319 \\ 2005 & 0.77 & 54.9 & 2400.41 & 9.9 & 363 \\ 2006 & 0.82 & 58.2 & 2534.48 & 10.6 & 383 \\ 2007 & 0.94 & 66.3 & 2922.20 & 11.9 & 441 \\ 2008 & 1.03 & 71.8 & 3226.40 & 12.7 & 483 \\ 2009 & 0.94 & 66.2 & 2963.86 & 12.0 & 440 \\ 2010 & 0.88 & 62.8 & 2783.93 & 11.7 & 411\end{array}$

TIME AFTER SHUTDOWB, YEARS

\begin{tabular}{|c|c|c|c|c|c|c|c|}
\hline $\begin{array}{l}\text { Volume } \\
\text { Thousands } \\
\left.\text { of } \mathrm{m}^{3}\right)\end{array}$ & $\begin{array}{c}\text { Actinide } \\
\text { Mass } \\
\text { (MT) }\end{array}$ & $\begin{array}{l}\text { Radio- } \\
\text { activity } \\
\text { (MCi) }\end{array}$ & $\begin{array}{c}\text { Therma I } \\
\text { Power } \\
\text { (MV) }\end{array}$ & $\begin{array}{l}\text { Haz } \\
\text { Met } \\
\text { Air }\end{array}$ & $\begin{array}{l}\text { zard, } \\
\text { ters } \\
\text { r }\end{array}$ & $\begin{array}{l}\text { Cubic } \\
\text { at RCG } \\
\text { water }\end{array}$ & \\
\hline $\begin{array}{l}0.00 \\
0.00 \\
0.04 \\
0.17 \\
0.30 \\
0.43 \\
0.60 \\
0.85 \\
1.11 \\
1.41 \\
1.79 \\
2.22 \\
2.73 \\
3.28 \\
3.92 \\
4.60 \\
5.38 \\
6.19 \\
7.13 \\
9.16 \\
9.10 \\
9.98\end{array}$ & $\begin{array}{r}0.0 \\
0.1 \\
2.9 \\
11.3 \\
19.8 \\
28.4 \\
39.8 \\
57.0 \\
74.5 \\
95.4 \\
122.0 \\
151.4 \\
186.8 \\
224.8 \\
268.9 \\
316.7 \\
371.6 \\
429.8 \\
496.1 \\
567.9 \\
634.1 \\
696.9\end{array}$ & $\begin{array}{r}1 \\
2 \\
100 \\
392 \\
706 \\
1052 \\
1541 \\
2267 \\
2955 \\
3752 \\
4765 \\
5872 \\
7218 \\
8670 \\
10361 \\
12156 \\
14196 \\
16312 \\
18756 \\
21435 \\
23776 \\
25877\end{array}$ & $\begin{array}{r}0.0 \\
0.0 \\
0.3 \\
1.4 \\
2.4 \\
3.6 \\
5.3 \\
7.8 \\
10.4 \\
13.5 \\
17.4 \\
21.6 \\
26.8 \\
32.1 \\
38.5 \\
45.6 \\
53.9 \\
62.7 \\
72.5 \\
82.7 \\
91.9 \\
100.6\end{array}$ & $\begin{array}{l}1.8 \mathrm{E} \\
5.2 \mathrm{E} \\
3.9 \mathrm{E} \\
1.3 \mathrm{E} \\
2.3 \mathrm{E} \\
3.5 \mathrm{E} \\
5.0 \mathrm{E} \\
8.4 \mathrm{E} \\
1.3 \mathrm{E} \\
2.1 \mathrm{E} \\
3.0 \mathrm{E} \\
3.9 \mathrm{E} \\
5.1 \mathrm{E} \\
6.1 \mathrm{E} \\
7.4 \mathrm{E} \\
9.3 \mathrm{E} \\
1.2 \mathrm{E} \\
1.4 \mathrm{E} \\
1.7 \mathrm{E} \\
1.9 \mathrm{E} \\
2.2 \mathrm{E} \\
2.5 \mathrm{E}\end{array}$ & $\begin{array}{l}17 \\
17 \\
18 \\
19 \\
19 \\
19 \\
19 \\
19 \\
20 \\
20 \\
20 \\
20 \\
20 \\
20 \\
20 \\
20 \\
21 \\
21 \\
21 \\
21 \\
21 \\
21\end{array}$ & $\begin{array}{l}5.7 \mathrm{E} \\
1.6 \mathrm{E} \\
6.8 \mathrm{E} \\
2.6 \mathrm{E} \\
4.8 \mathrm{E} \\
7.1 \mathrm{E} \\
1.0 \mathrm{E} \\
1.5 \mathrm{E} \\
2.0 \mathrm{E} \\
2.5 \mathrm{E} \\
3.2 \mathrm{E} \\
3.9 \mathrm{E} \\
4.8 \mathrm{E} \\
5.8 \mathrm{E} \\
6.9 \mathrm{E} \\
8.0 \mathrm{E} \\
9.3 \mathrm{E} \\
1.1 \mathrm{E} \\
1.2 \mathrm{E} \\
1.4 \mathrm{E} \\
1.6 \mathrm{E} \\
1.7 \mathrm{E}\end{array}$ & $\begin{array}{l}11 \\
12 \\
13 \\
14 \\
14 \\
14 \\
15 \\
15 \\
15 \\
15 \\
15 \\
15 \\
15 \\
15 \\
15 \\
15 \\
15 \\
16 \\
16 \\
16 \\
16 \\
16\end{array}$ \\
\hline $\begin{array}{l}9.98 \\
9.98 \\
9.98 \\
9.98 \\
9.98 \\
9.98 \\
9.98 \\
9.98 \\
9.98\end{array}$ & $\begin{array}{l}696.9 \\
696.9 \\
696.9 \\
696.9 \\
696.9 \\
696.9 \\
696.9 \\
696.9 \\
696.9\end{array}$ & $\begin{array}{r}2422.5 \\
88.6 \\
27.0 \\
13.8 \\
8.7 \\
3.9 \\
2.0 \\
1.5 \\
0.8\end{array}$ & $\begin{array}{l}9.8 \\
1.7 \\
0.7 \\
0.3 \\
0.2 \\
0.1 \\
0.0 \\
0.0 \\
0.0\end{array}$ & $\begin{array}{l}5.3 \mathrm{E} \\
3.1 \mathrm{E} \\
1.3 \mathrm{E} \\
6.8 \mathrm{E} \\
4.5 \mathrm{E} \\
2.1 \mathrm{E} \\
4.1 \mathrm{E} \\
2.6 \mathrm{E} \\
2.7 \mathrm{E}\end{array}$ & $\begin{array}{l}20 \\
20 \\
20 \\
19 \\
19 \\
19 \\
18 \\
18 \\
18\end{array}$ & $\begin{array}{l}1.4 \mathrm{E} \\
2.2 \mathrm{E} \\
4.8 \mathrm{E} \\
1.8 \mathrm{E} \\
1.1 \mathrm{E} \\
4.7 \mathrm{E} \\
3.8 \mathrm{E} \\
4.7 \mathrm{E} \\
2.8 \mathrm{E}\end{array}$ & $\begin{array}{l}15 \\
13 \\
12 \\
12 \\
12 \\
11 \\
11 \\
11 \\
11\end{array}$ \\
\hline
\end{tabular}


Table 4.12. Grams of accumulated heavy elements in high-level wastes at a federal repository in the year 2010 as a function of age

\begin{tabular}{|c|c|c|c|c|c|c|c|c|}
\hline & Year & & & 드으르으느 & _clㅡ으므. & th $\underline{h} \underline{e}$ rer & 드드. & \\
\hline & 2010 & 100 & 300 & 1000 & 3000 & 10,000 & 100,000 & $1,000,000$ \\
\hline $\mathrm{E}$ & 05 & $.64 \mathrm{EC}$ & $55 \mathrm{E}$ 0드. & $1 \mathrm{E} 05$ & $2 \mathrm{E} \quad 05$ & $\mathrm{E}$ & 96 & $4 E$ \\
\hline & -07 & $E-07$ & $5 E-0 T$ & $E-07$ & $E-07$ & $E-07$ & $E-06$ & $77 \mathrm{E}-06$ \\
\hline & 01 & E 01 & $5 E \quad 01$ & $5.62 \mathrm{E} \quad 01$ & $0 \mathrm{E} \quad 02$ & $1 \mathrm{E} 03$ & $8 E 05$ & \\
\hline I & $g-02$ & $E-02$ & $.47 E-0$. & $3.30 \mathrm{E} 00$ & $22 E \quad 01$ & $5 \pm 03$ & $6 \geq 05$ & $9 \mathrm{E}$ \\
\hline 0 & $-6+2+2$ & $2+2+3$ & $.10 E-04$ & $E-03$ & $E-02$ & $E-01$ & $8 \mathrm{E} 00$ & $E-C$ \\
\hline $\mathrm{T}$ & - & 6 & $11 E-12$ & $e-11$ & 11 & -1 & $E-08$ & $-0+0$ \\
\hline & 2 & & $8 E-06$ & -05 & 4 & 03 & $E-02$ & $E-C$ \\
\hline & & & $E-$ & & 7 & & .04 & -0 \\
\hline A & 2 & 1 & $6 \mathrm{E}$ & 1 & 02 & & 03 & $\mathrm{E}$ \\
\hline C & 4 & 9 & $\mathrm{E}$ & -01 & 1 & 8 & E 00 & $E$ \\
\hline $\mathrm{H}$ & 3. & 3. & $3.65 \mathrm{E} \quad 05$ & E 05 & $\begin{array}{ll}E & 05\end{array}$ & 05 & E 05 & $2 \mathrm{E} 06$ \\
\hline & $1.55 \mathrm{E}$ & 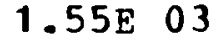 & $1.54 \mathrm{E} \quad 03$ & $1.52 \mathrm{E} \quad 03$ & $1.47 E 03$ & 1. $30 \mathrm{E} 0$ & $8 \mathrm{E} 03$ & $9 \mathrm{E}$ \\
\hline ठ & $2 \mathrm{E} 08$ & $2 \mathrm{E} \quad 08$ & $5.13 E 03$ & $5.14 \mathrm{E} \quad 08$ & $5.17 \mathrm{E} \quad 08$ & $5.24 \mathrm{E} \quad 08$ & $34 \mathrm{E} \quad 08$ & $8 \mathrm{E} \quad 08$ \\
\hline $\mathbf{P}$ & $\begin{array}{ll}4 \mathrm{E} \quad 07 \\
\end{array}$ & $\begin{array}{ll}3 E & 07\end{array}$ & $06 \mathrm{E} \quad 07$ & $7.74 \mathrm{E} \quad 07$ & $8.07 \mathrm{E} \quad 07$ & $8.12 \mathrm{E} 07$ & $6 \mathrm{E} 07$ & $4 E$ \\
\hline & $1.18 \mathrm{E} \quad 07$ & E 07 & $39 \mathrm{E} \quad 07$ & $1.74 \mathrm{E} \quad 07$ & $1.90 \mathrm{E} 07$ & $2 \mathrm{E} 0$ & $2 \mathrm{E} 06$ & \\
\hline & $4.35 E 07$ & $1 \mathrm{E} \quad 07$ & $6 \mathrm{E} 07$ & $2.80 \mathrm{E} 07$ & $\begin{array}{ll}7 \mathrm{E} & 07\end{array}$ & E 07 & $9 E \quad 03$ & $3 E$ \\
\hline & 06 & 06 & 05 & 06 & 06 & 05 & $\begin{array}{ll}3 \mathrm{E} & 03\end{array}$ & $+E$ \\
\hline & $z-0 L$ & -14 & $8 E-14$ & $E-14$ & $E-14$ & $E-14$ & $36 E-16$ & $2 E$ \\
\hline & E 0 & $\begin{array}{ll}E & 0\end{array}$ & E $O D$ & $E-01$ & $+E-02$ & $E-04$ & $3 E-11$ & \\
\hline & OE 08 & $0 E$ & 10B 08 & 10E 08 & 10E 08 & $10 \mathrm{E} 08$ & $40 \mathrm{E} 08$ & $40 \mathrm{E}$ \\
\hline
\end{tabular}


Table 4.13. Curies of accumulated heavy isotopes in high-level wastes at a federal repository in the year 2010 as a function of age

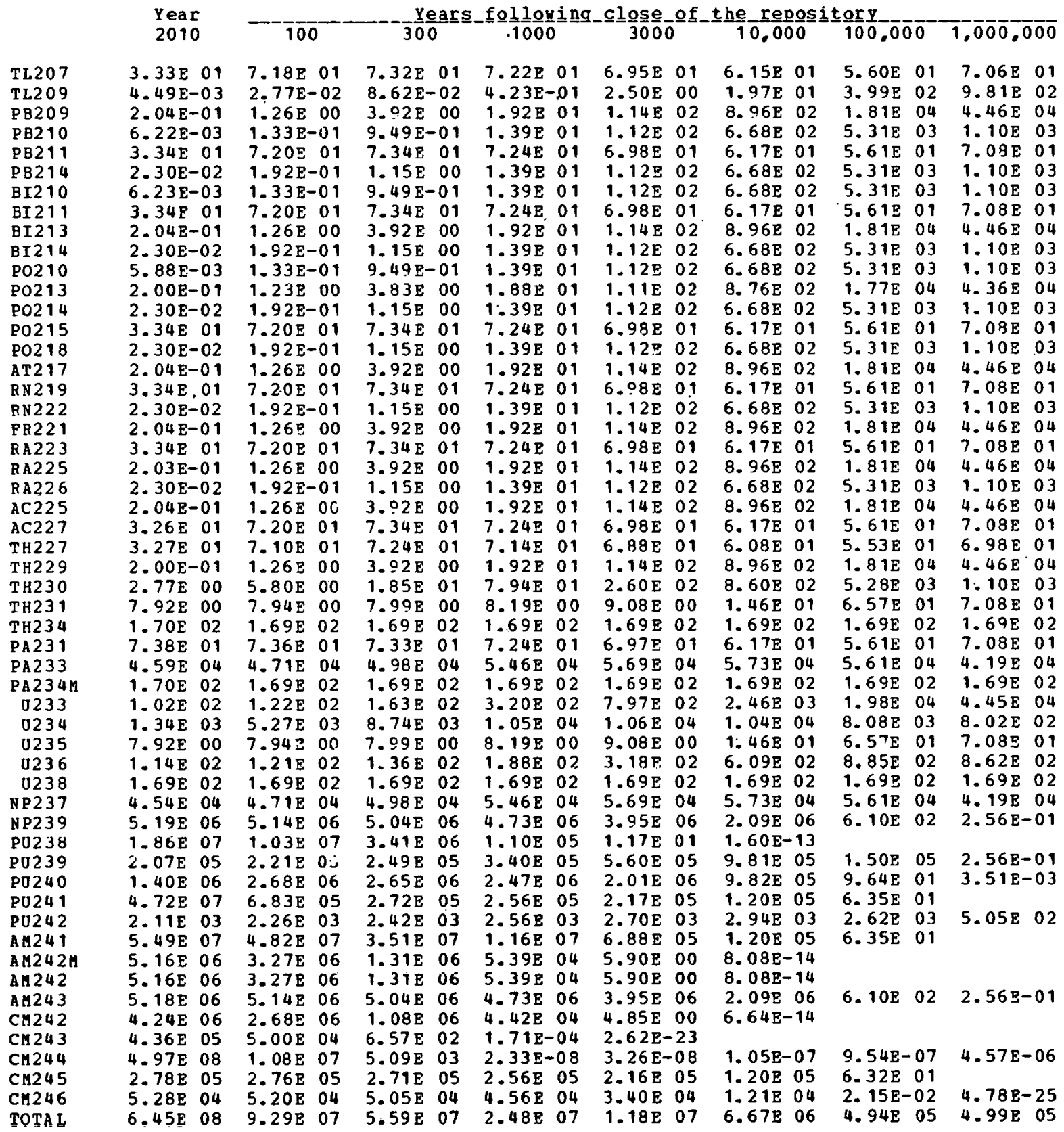


Table 4. 14. Matts of accumulated heavy isotopes in high-level wastes at a federal repository in the year 2010 as a function of age

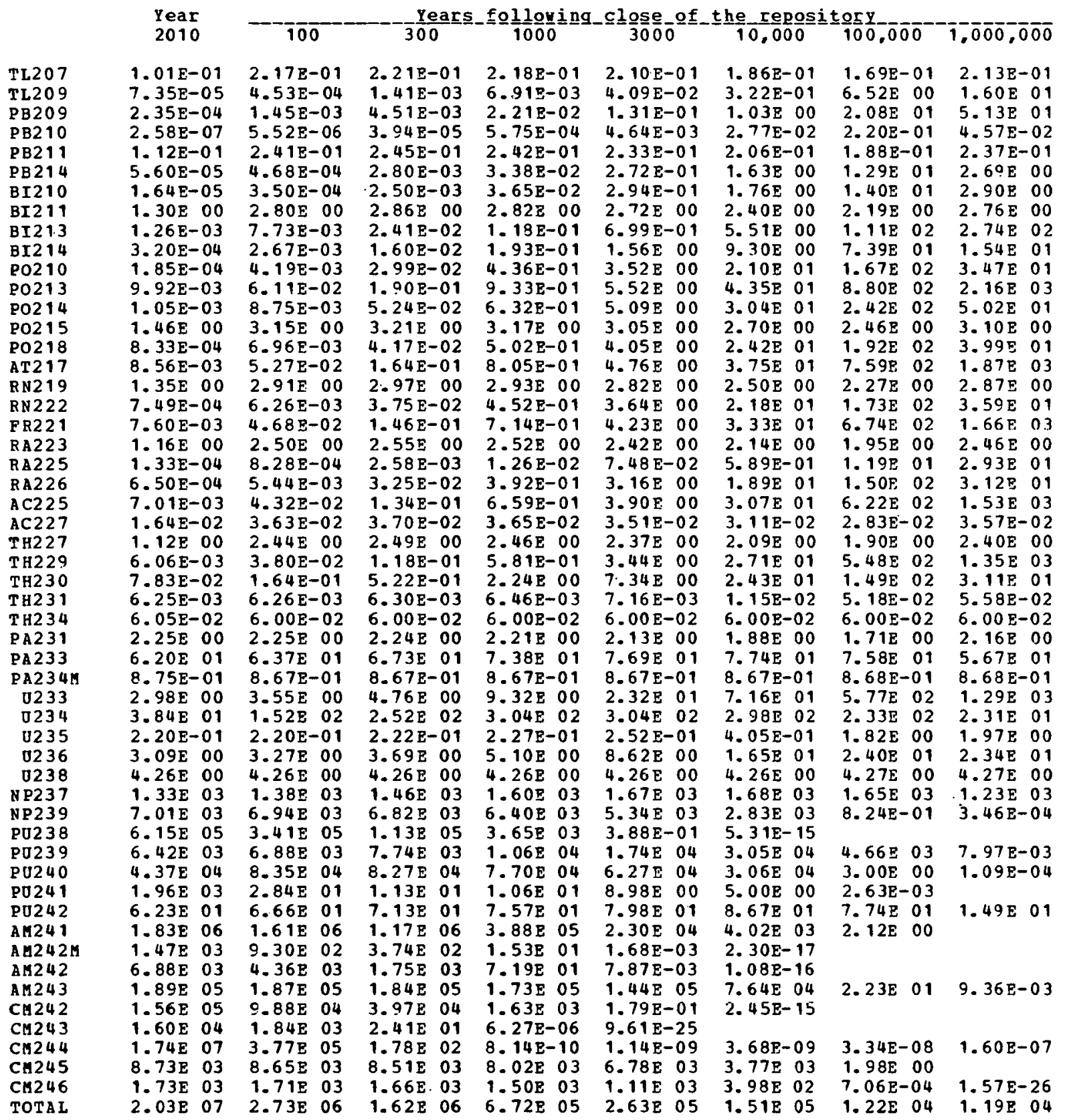


Table 4.15. Ingestion toxicity $\left(\mathrm{m}^{3}\right.$ water) of accumulated fission product isotopes in high-level wastes at a federal repository in the year 2010 as a function of time

\begin{tabular}{|c|c|c|c|c|c|c|c|c|c|c|c|c|c|c|c|c|}
\hline & Year & & & & & & 11004 & & $30+2-$ & & & & & & & \\
\hline & & & 100 & & 300 & & 1000 & & & & 10. & & 10 & & 0 & ) \\
\hline $20^{\circ}-x-1$ & $4.17 \mathrm{E}$ & 03 & $\mathrm{E}$ & 03 & tE & 03 & $3 \mathrm{E}$ & 03 & $E$ & 03 & $7.69 \mathrm{E}$ & 03 & $7.00 \mathrm{E}$ & 03 & $8.83 \mathrm{E}$ & \\
\hline L20 & & -01 & & 0 & $62 \mathrm{E}$ & 00 & & & & 2 & & & & 4 & & \\
\hline 10 & $81 \mathrm{E}$ & r & $9 \mathrm{E}$ & 02 & & 03 & & & & 04 & & 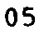 & & 6 & $9 \mathrm{~F}$ & 07 \\
\hline B2 & $22 \mathrm{E}$ & 04 & & 06 & $49 \mathrm{E}$ & 06 & & 08 & $1.12 \mathrm{E}$ & 09 & $8 \mathrm{E}$ & 09 & 318 & 10 & $\mathrm{OE}$ & 0 \\
\hline & $36 \mathrm{E}$ & 04 & $\mathrm{OE}$ & 05 & $33 E$ & 05 & $1 \mathrm{E}$ & 05 & $1.74 \mathrm{E}$ & 0 & & & $O E$ & 05 & & \\
\hline & & 01 & & 02 & $O E$ & 03 & & 04 & & 05 & & 06 & & 07 & $O E$ & 06 \\
\hline & $6 E$ & 02 & & 03 & & 04 & 68 & & $79 \mathrm{E}$ & 06 & & c & & 08 & & 07 \\
\hline & & 03 & & 04 & $5 E$ & 04 & & 04 & $.96 \mathrm{E}$ & 03 & IE & & & 3 & & 04 \\
\hline & & 02 & & 03 & & 03 & & 04 & & 05 & & & & 07 & & 07 \\
\hline & & 01 & & 02 & & 03 & & 04 & $\mathrm{E}$ & 0 & & 06 & & 6 & & 06 \\
\hline $02+2+2$ & $8.40 \mathrm{E}$ & 03 & $E$ & 05 & $6 \mathrm{E}$ & 06 & & 07 & E & & & 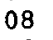 & & 09 & $7 \mathrm{E}$ & 09 \\
\hline & $2.00 \mathrm{~F}-$ & -01 & & 00 & & 00 & & 01 & $1.11 \mathrm{E}$ & 02 & $6 E$ & 02 & & 04 & & 04 \\
\hline & & -02 & & .01 & $5 \mathrm{E}$ & 00 & & 01 & $1.12 \mathrm{E}$ & 02 & 6. & 02 & & 3 & & 03 \\
\hline & $4 \mathrm{E}$ & 01 & & 01 & 45 & 01 & & 01 & $\mathrm{E}$ & 01 & & & & 1 & & 01 \\
\hline & $.75 \mathrm{E}$ & 00 & & 01 & $37 \mathrm{E}$ & 02 & & 03 & & 04 & & 05 & & 6 & & 05 \\
\hline & $3.4-2$ & -01 & & 00 & $3 E$ & 00 & $3.20 \mathrm{E}$ & 01 & $.89 \mathrm{E}$ & 02 & 1. & ( & & 4 & & .04 \\
\hline $2 \mathrm{N2}$ & 3.3 & 01 & 7. & 01 & $4 \mathrm{E}$ & 01 & 7.2 & 01 & 6.9 & 01 & & & & 1 & & 01 \\
\hline & & -02 & & -01 & $5 \mathrm{E}$ & 00 & & 01 & & 02 & & c & & 3 & & 03 \\
\hline & & 02 & & 03 & $\mathrm{OE}$ & 03 & 2. & 04 & $.42 E$ & 0 & & & & 7 & & 07 \\
\hline &. & 07 & & 08 & & 08 & & 08 & $9.96 \mathrm{~F}$ & 0 & & 0 & & 07 & & 08 \\
\hline & & 05 & & 06 & & 06 & & 07 & 2. & 0 & & 0 & & 10 & & 10 \\
\hline & $5 \mathrm{E}$ & 05 & $\mathbf{E}$ & 06 & $83 E$ & 07 & $4.62 E$ & 08 & $\mathrm{E}$ & & & & & 11 & & 10 \\
\hline & $.09 \mathrm{E}$ & 04 & & 05 & $4 \mathrm{E}$ & 0 & & 0 & $E$ & 07 & & c & & 9 & & 9 \\
\hline & $63 \mathrm{E}$ & 07 & & 07 & $7 \mathrm{E}$ & 07 & & 0 & $3.49 \mathrm{E}$ & 0 & 3. & 07 & & 7 & & 07 \\
\hline & $E$ & 06 & & 06 & $62 \mathrm{E}$ & 06 & & 06 & $3.44 \mathrm{E}$ & 0 & & & & 6 & & 06 \\
\hline & $1 \mathrm{E}$ & 05 & & 06 & E & 06 & & 07 & $2.84 \mathrm{E}$ & 0 & & & & 10 & & 11 \\
\hline & & 06 & & 06 & $3 \mathrm{E}$ & 06 & & 07 & $1.30 \mathrm{E}$ & 0 & & ( & & 9 & & 08 \\
\hline & $96 \mathrm{E}$ & 04 & & 04 & $9 \mathrm{E}$ & 04 & $\mathbf{E}$ & 04 & $4.54 \mathrm{E}$ & 0 & & & & 5 & & 05 \\
\hline & & 06 & & 0 & & 06 & & 0 & $E$ & 06 & & o & & 6 & & 06 \\
\hline & & 07 & & 0 & & 0 & & 0 & E & 07 & & ( & & 07 & & 07 \\
\hline & $.59 \mathrm{E}$ & 08 & & 08 & & 0 & & 0 & & 0 & & & & 0 & & 08 \\
\hline & & 03 & & 0 & & 0 & & 0 & $E$ & 0. & 8. & 0 & $3 E$ & 03 & E & 03 \\
\hline & $\mathbf{F}$ & 06 & & 0 & & 0 & 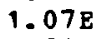 & 0 & $E$ & 0 & & & & 08 & & 09 \\
\hline & & 07 & & 0 & & 0 & & 0 & & 0 & & & & & & 07 \\
\hline & & 05 & & 0 s & & 0 & & 0 & & 0 & & & & 06 & & 06 \\
\hline & & 06 & & 06 & & 0 & & 0 & & 0 & & 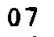 & & 0 & & 07 \\
\hline & & 0 & & 0 & & 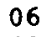 & & 0 & & & & & & 06 & & 06 \\
\hline & & 10 & & 1 & & 1 & & 1 & & 1 & 1. & 1 & & 10 & & 10 \\
\hline & & 10 & & 1 & & 1 & & 1 & & 1 & & 10 & & re & & 03 \\
\hline & & 12 & & 1 & & 11 & & 1 & & 0 & & & & & & \\
\hline & & 10 & & 10 & & 10 & 6. & 10 & E & 11 & 1. & 11 & & 10 & & 04 \\
\hline & & 11 & & 1 & & 11 & & 1 & & 11 & & 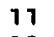 & & & & \\
\hline & & 11 & & 09 & & 0 & & 0 & & 0 & & & & 05 & & \\
\hline & $4.22 \mathrm{E}$ & 08 & & 08 & & 0 & & & & 08 & & 08 & $4 \mathrm{E}$ & 08 & $1.01 \mathrm{E}$ & 08 \\
\hline & & 13 & & 13 & & 12 & & 1 & & 11 & & 10 & & 07 & & \\
\hline & & 12 & & 11 & & 11 & & 10 & & & & & & & & \\
\hline & & 10 & & 10 & & 1 & & 0 & & 04 & & 10 & & & & \\
\hline & & 12 & & 12 & $1.26 \mathrm{E}$ & 12 & & 1 & $9.87 E$ & 11 & & 11 & $.52 E$ & 0 ? & $1 \mathrm{E}$ & 04 \\
\hline & & 11 & & 11 & & 10 & & & & & & & & & & \\
\hline & & 10 & & 10 & & 08 & & 01 & & -18 & & & & & & \\
\hline & $9 \mathrm{E}$ & 13 & $1.54 \mathrm{E}$ & 12 & $7.27 \mathrm{E}$ & 08 & & -03 & $66 \mathrm{E}-$ & -03 & & & & 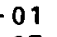 & $5 ?$ 5 & \\
\hline & & 16 & & 10 & & 10 & & 1 & & & & & & 07 & & \\
\hline & $2 E$ & 10 & & 10 & & 10 & & 10 & $\mathbf{F}$ & 0 & $3.03 \mathrm{E}$ & 09 & $5.38 \mathrm{E}$ & 03 & - & -1 \\
\hline UTAL & $9.20 \mathrm{E}$ & 15 & $1.81 \mathrm{E}$ & 13 & $1,18 \mathrm{E}$ & 13 & $4.83 \mathrm{E}$ & 12 & $1.80 \mathrm{E}$ & 12 & $1.06 \mathrm{E}$ & 12 & $3.77 \mathrm{E}$ & 11 & $2.76 \mathrm{E}$ & \\
\hline
\end{tabular}


Table 4.16. Grams of accumulated fission product elements in high-level wastes at a federal repository in the year 2010 as a function of age

\begin{tabular}{|c|c|c|c|c|c|c|c|c|c|c|c|c|c|c|c|c|}
\hline & 2 & & & & & & & & & & & & & & & \\
\hline & 20 & & 100 & & 300 & & 10 & 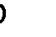 & 30 & & 10. & & 100,00 & & 1.000 & \\
\hline$\varepsilon$ & $25 \mathrm{E}$ & 04 & $E$ & 04 & $5 \mathrm{E}$ & 04 & $4.25 \mathrm{E}$ & 04 & $.25 \mathrm{E}$ & 04 & $4.25 \mathrm{E}$ & 04 & $4.25 E$ & 04 & $25 \mathrm{E}$ & \\
\hline & & & & & & & & 0 & & & & & $\mathbf{E}$ & & & \\
\hline$E$ & & 06 & & 06 & & 06 & & 06 & & 06 & & 06 & $2 \mathrm{E}$ & 06 & & \\
\hline & & 0 & & 0 & $2 \mathrm{E}$ & 0 & & 0 & $O E$ & 04 & & 04 & & 05 & & \\
\hline 8 & & 07 & & 0 & & 07 & & 07 & & 07 & & 07 & & & & \\
\hline $\mathbf{R}$ & & 07 & & 07 & & 07 & & 07 & & 07 & & 07 & & 07 & & \\
\hline $\mathbf{Y}$ & & 0 & & 0 & $5 \mathrm{E}$ & 07 & & 07 & $5 \mathrm{E}$ & 07 & & 07 & & 7 & & \\
\hline$R$ & & 08 & & 0 & & 08 & & 0 & & 0 & & & & 0 & & \\
\hline B & & 02 & & 0 & $4 \mathrm{E}$ & 04 & & 04 & & 4 & & 0 & & 0 & & \\
\hline 0 & & 08 & & 0 & $7 \mathrm{E}$ & 08 & & 0 & & 0 & & & & 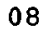 & & \\
\hline C & & 07 & & 0 & & 0 & & 0 & & 0 & & & & 07 & & \\
\hline U & & 08 & & $0 E$ & & 08 & & 0 & $9 E$ & 0 & & 0 & & 0 & & \\
\hline $\mathrm{H}$ & & 07 & 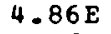 & 07 & $6 \mathrm{E}$ & 07 & & 0 & 4 & 0 & & & & & & \\
\hline & & 08 & & 08 & & 0 & & 08 & & 08 & & 0 & & 08 & & \\
\hline & & 06 & & 0 & & 0 & & 0 & & 06 & & 0 & & & & \\
\hline CD & & 07 & & 0 & & 07 & & 0 & & 0 & & 0 & & & & \\
\hline IN & & 05 & & 0 s & & 0 & & 05 & & 0 & & 0 & & & & \\
\hline N & & 06 & & 0 & & 0 & & 06 & & 06 & & 0 & & 0 & & \\
\hline SB & & 05 & & 05 & & 05 & & 0 & & 0 & & 0 & & 0 & & \\
\hline$F$ & & 07 & & 07 & & 07 & & 07 & & 0 & & & & & & \\
\hline$I$ & & 04 & & 0 & & 04 & & 04 & & 04 & & 0 & & 0 & & \\
\hline 8 & & -02 & & -01 & & 01 & & 0 & & 00 & & 01 & & 02 & & \\
\hline Cs & & 08 & & 0 & & 0 & & 0 & & 0 & & 08 & & & & \\
\hline A & & 08 & & 08 & & 08 & & 0 & & 08 & & 0 & & 0 & & \\
\hline LA & & 08 & & 08 & & 0 & & 0 & & 08 & & 08 & & 0 & & \\
\hline$C$ & & 08 & & 0 & & 0 & & 08 & & 0 & & & & & & \\
\hline PR & & 08 & & $0 \varepsilon$ & & 0 & & 0 & & 0 & & & & & & \\
\hline ND & & 08 & & 08 & $7 \mathrm{E}$ & 0 & & 0 & & 0 & & & & & & \\
\hline PG & & 05 & & & & & & & & & & & & & & \\
\hline SH & & 07 & & 07 & & 0 & & 0 & & 0 & & & & & & \\
\hline EU & & 07 & & 0 & & 0 & & 0 & & $\hat{0}$ & & & & & & \\
\hline GD & & 07 & & 07 & & 0 & & 0 & & 0 & & 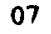 & & & & \\
\hline$T B$ & & 05 & & 05 & & 05 & & 05 & & 0 & & o & & & & \\
\hline$D Y$ & & 05 & & 0 & & 05 & & 05 & & 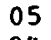 & & 05 & & 5 & & \\
\hline 4 & & 0 & & 0 & & 0 & & $\dot{0}$ & & 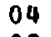 & & 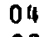 & & 011 & & \\
\hline & & 03 & & 0 & & 0 & & 03 & & 03 & & 03 & & 0.3 & & \\
\hline & & . & & . & & 09 & & 0 & & 0 & & 9 & IE & 09 & $1 \mathrm{E}$ & \\
\hline
\end{tabular}


Table 4.17. Curies of accumulated fission product isotopes in high-level wastes at a federal repository in the year 2010 as a function of age

\begin{tabular}{|c|c|c|c|c|c|c|c|c|c|c|c|c|}
\hline & \multicolumn{2}{|c|}{ Year } & \multicolumn{10}{|c|}{ Years } \\
\hline & \multicolumn{2}{|c|}{2010} & 100 & \multicolumn{2}{|c|}{300} & $=\underline{10} \frac{1}{10} \_00$ & $\therefore \perp \frac{0}{30} \leq 00$ & \multicolumn{2}{|c|}{10,000} & \multicolumn{2}{|c|}{100.000} & $\overline{1}, \overline{0} \overline{0} \overline{0}, \overline{0} \overline{0} \overline{0}$ \\
\hline SE 79 & 3.915 & 04 & $3.90 \mathrm{E} 04$ & $3.89 \mathrm{E}$ & 04 & $3.37 \mathrm{E} \quad 04$ & $3.78 \mathrm{E} \quad 04$ & 3. $51 \mathrm{E}$ & 04 & $1.35 \mathrm{~F}$ & 04 & $9.13 \mathrm{E}-01$ \\
\hline$S R 90$ & $4.83 \mathrm{E}$ & 09 & 4. 1OE OE & $2.96 \mathrm{~F}$ & 06 & $9.37 \mathrm{E}-02$ & $3.51 \mathrm{E}-23$ & & & & & \\
\hline Y 90 & 4. $84 \mathrm{E}$ & 09 & 4. $10 \mathrm{E}$ OE & $6 \mathrm{E}$ & 06 & $7 E-02$ & $1 F-23$ & & & & & \\
\hline ZR 93 & $.20 E$ & 05 & $1.80 \mathrm{E} \quad 05$ & $80 \mathrm{E}$ & 05 & $0=05$ & $80 \mathrm{E} \quad 05$ & $1.79 \mathrm{E}$ & 05 & 1. $72 \mathrm{E}$ & 05 & $1.13 \mathrm{E} 05$ \\
\hline NB $93 M$ & $12 E$ & 05 & $1.80 E \quad 05$ & $O E$ & 05 & $10 E 05$ & $1.80 \mathrm{E} 05$ & $1.79 \mathrm{E}$ & 05 & $72 \pm$ & 05 & $3 \mathrm{E} 05$ \\
\hline TC 99 & $1.46 \mathrm{E}$ & 06 & $1.46 \mathrm{P} 06$ & $1.46 \mathrm{E}$ & 06 & $5 E \quad 06$ & $1.44 \mathrm{~F} 06$ & $1.41 \mathrm{E}$ & 06 & $5 \mathrm{~F}$ & 06 & $+8 \quad 04$ \\
\hline 20106 & $6 . \mathrm{CIE}$ & 06 & & & & & & & & & & \\
\hline RH 1106 & $6.01 \mathrm{E}$ & 06 & & & & & & & & & & \\
\hline PD107 & $1.24 E$ & 04 & $1.24 \mathrm{E} \quad 04$ & $1.24 E$ & 04 & $1.24 \mathrm{E} \quad 04$ & $1.24 \mathrm{E} \quad 04$ & $1.24 E$ & 04 & 1.235 & 04 & $1.12 \mathrm{E} \quad 04$ \\
\hline SB125 & $2.53 \mathrm{E}$ & 07 & 1. $80 E-04$ & & & & & & & & & \\
\hline TE125H & $1.05 \mathrm{E}$ & 07 & $7.44 E-05$ & & & & & & & & & \\
\hline $\operatorname{SN126}$ & $6.51 \mathrm{E}$ & 04 & $6.50 \mathrm{E} \quad 04$ & $6.49 \mathrm{E}$ & 04 & $6.46 \mathrm{E} \quad 04$ & $6.37 \mathrm{E} \quad 04$ & $6.07 \mathrm{E}$ & 04 & $3.25 E$ & 04 & $6.36 \mathrm{E} \quad 01$ \\
\hline SE126M & $6.51 \mathrm{E}$ & 04 & $6.50 E \quad 04$ & $6.49 \mathrm{E}$ & 04 & $6.46 \mathrm{E} 04$ & $6.37 \mathrm{E} 04$ & $6.07 \mathrm{E}$ & 04 & $3.25 \mathrm{E}$ & 04 & $5 \mathrm{E} \quad 01$ \\
\hline SB126 & $6.47 \mathrm{E}$ & 04 & $6.44 \mathrm{E} \quad 04$ & $6.43 \mathrm{E}$ & 04 & $6.40 E 04$ & $6.31 \mathrm{~F} \quad 04$ & $6.01 \mathrm{E}$ & 04 & 3. $22 \mathrm{~F}$ & 04 & $0 \mathrm{~F} 01$ \\
\hline I129 & $4.86 \mathrm{E}$ & 00 & $4.86 E \quad 00$ & $4.86 E$ & 00 & $4.86 E \quad 00$ & $4.86 \mathrm{E} \quad 00$ & $4.86 \mathrm{E}$ & 00 & $4.84 E$ & 00 & $4.67 \mathrm{E} \quad 00$ \\
\hline $\operatorname{cs} 13$ & 1. & 08 & $4.03 E-07$ & & & & & & & & & \\
\hline $\operatorname{cs} 135$ & $3.50 \mathrm{E}$ & 04 & 3.50504 & $3.50 \mathrm{E}$ & 04 & $3.50 \mathrm{E} \quad 04$ & $3.50 \mathrm{E} 04$ & $3.49 \mathrm{E}$ & 04 & $3.42 \mathrm{E}$ & 04 & $2.78 \mathrm{~F} \quad 04$ \\
\hline $\operatorname{cs} 13$ & $7.56 \mathrm{E}$ & 09 & $7.50 \mathrm{E} \quad 08$ & 7. $38 \mathrm{E}$ & 06 & $6.98 E-01$ & $5.96 E-21$ & & & & & \\
\hline BA 1.3 & $7.07 \mathrm{~F}$ & 09 & $7.01 \mathrm{~F} \quad 08$ & $6.90 \mathrm{E}$ & 06 & $6.53 F-01$ & $5.57 \mathrm{E}-21$ & & & & & \\
\hline PM14 & $2.19 \mathrm{E}$ & 08 & $7.09 E-04$ & & & & & & & & & \\
\hline SM151 & $1.13 E$ & 08 & $5.11 \mathrm{E} \quad 07$ & $1.04 \mathrm{E}$ & 07 & $3.90 \mathrm{E} 04$ & $4.69 E-03$ & & & & & \\
\hline EU152 & $6.03 \mathrm{E}$ & 05 & $1.87 E \quad 03$ & $1.80 \mathrm{E}$ & 02 & $4.96 E-20$ & & & & & & \\
\hline FU154 & $3.43 \mathrm{~F}$ & 08 & $4.51 \mathrm{~F}: 06$ & $7.79 \mathrm{E}$ & 02 & 5. $26 E-11$ & & & & & & \\
\hline EU 155 & $3.95 \mathrm{E}$ & 06 & $9.21 \mathrm{E}-11$ & & & & & & & & & \\
\hline H0166M & $1.03 \mathrm{E}$ & 02 & $9.74 \mathrm{E} \quad 01$ & $\varepsilon .68 \mathrm{E}$ & 01 & $5.7^{\circ} \mathrm{E} 01$ & $1.82 \mathrm{E} \quad 01$ & 3.2 & .01 & & -24 & \\
\hline TOTAL & $2.52 \mathrm{E}$ & 10 & $2.33 \mathrm{E} 09$ & $3.27 \mathrm{E}$ & 07 & $13 E \quad 06$ & $08 \mathrm{E} \quad 06$ & $2.03 \mathrm{E}$ & 06 & $1.55 \mathrm{E}$ & 06 & $3.21 \mathrm{E} \quad 05$ \\
\hline
\end{tabular}


Table 4.18. Watts of accumulated fission product isotopes in high-level wastes at a federal repository in the year 2010 as a function of age

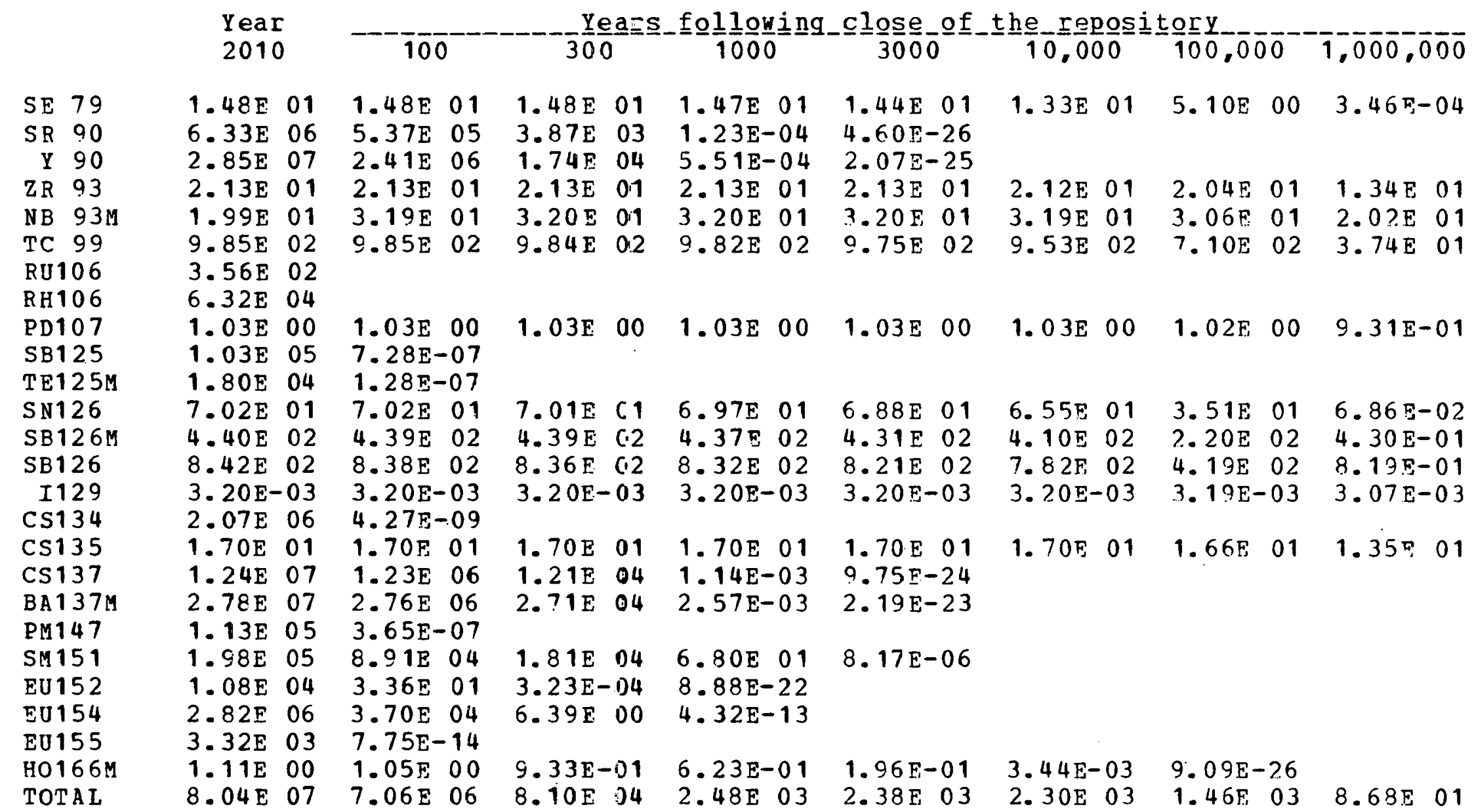


Table 4.19. Ingestion toxicity ( ${ }^{3}$ water) of accumulated fission product isotopes in high-level wastes at a federal repository in the

year 2010 as a function of age

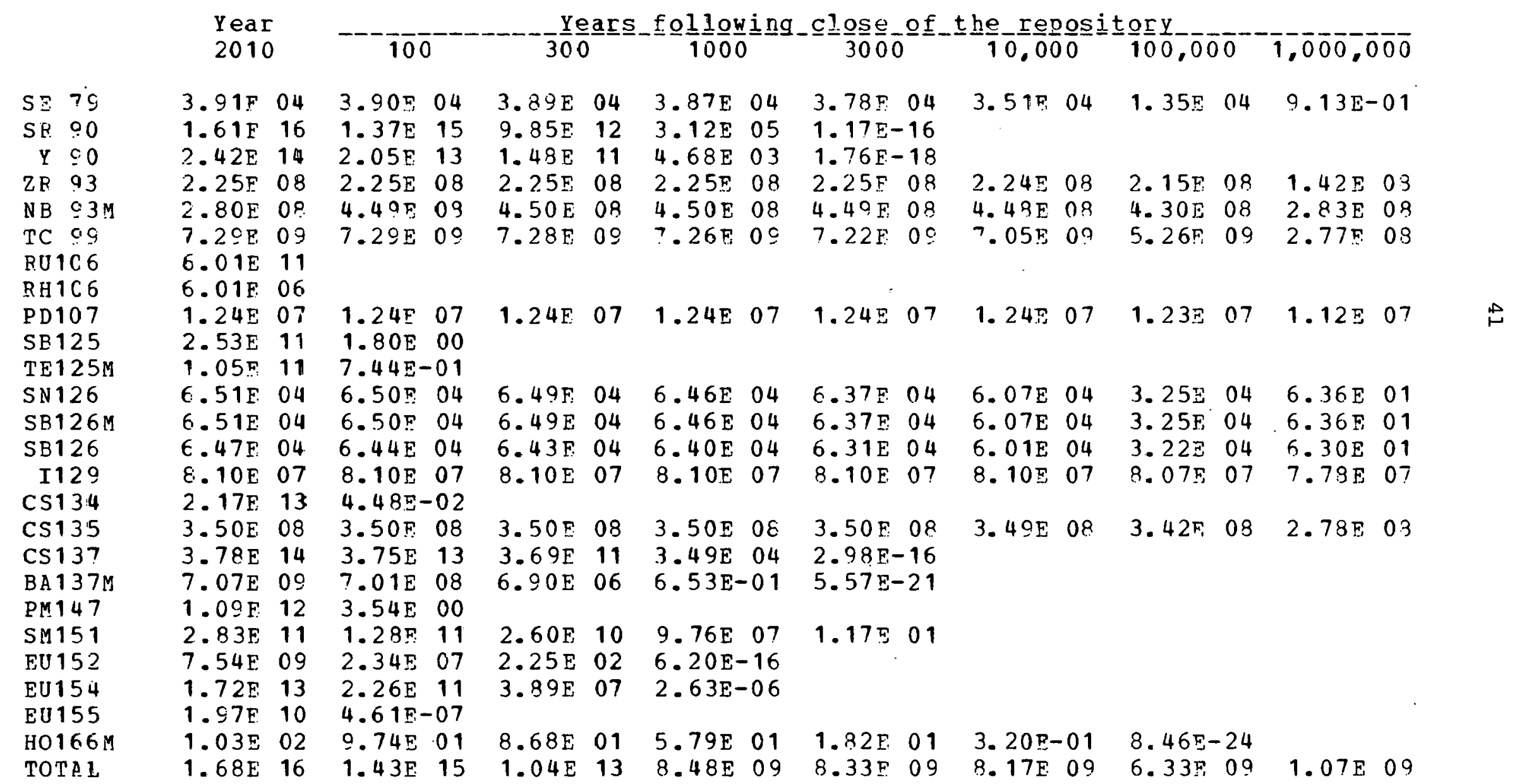


Table 4.20. Mass, composition, and exposure of fuel assembly structural materials

\begin{tabular}{|c|c|c|c|c|c|c|}
\hline \multirow[b]{2}{*}{$\begin{array}{l}\text { Structural } \\
\text { material }\end{array}$} & \multicolumn{2}{|c|}{ PWR } & \multicolumn{2}{|c|}{ BWR } & \multicolumn{2}{|c|}{ LMFBR } \\
\hline & $\operatorname{Mass}^{\mathrm{a}}{ }_{\left(\mathrm{kg} / \mathrm{MTHM}^{\mathrm{b}}\right)}$ & $\begin{array}{c}\text { Percentage } \\
\text { exposure }^{C}\end{array}$ & $\begin{array}{c}\text { Mass }^{\mathrm{d}} \\
(\mathrm{kg} / \mathrm{MTHM})\end{array}$ & $\begin{array}{c}\text { Percentage } \\
\text { exposure }\end{array}$ & $\begin{array}{c}\text { Mass }^{\mathrm{e}} \\
(\mathrm{kg} / \mathrm{MTHM})\end{array}$ & $\begin{array}{c}\text { Percentage } \\
\text { exposure }\end{array}$ \\
\hline Zircaloy & 235.0 & 100 & 316.0 & 100 & 0 & - \\
\hline 302 sS & 4.2 & 60 & 0 & - & 0 & - \\
\hline 304 SS & 37.1 & 40 & 50.0 & 50 & 0 & - \\
\hline $316 \mathrm{sS}$ & 0 & - & 0 & - & 700.0 & 100 \\
\hline Inconel & 12.8 & 100 & 3.4 & 100 & 0 & - \\
\hline Nicrobraze 50 & 2.6 & 100 & 0 & - & 0 & - \\
\hline Total & 291.7 & - & 369.4 & - & 700.0 & 100 \\
\hline
\end{tabular}

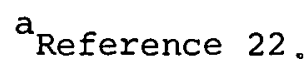

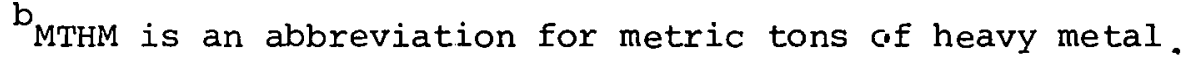

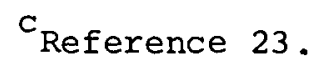

$\mathrm{d}_{\text {Reference } 24 .}$

e Reference 1 . 
is assumed to contain $0.05 \%$ of the actinides and fission products originally in the spent fuel. The radioactivity and thermal power of the major actinide and fission-product isotopes accompanying the structural materials associated with one metric ton of each type of reactor fuel are also summarized in Appendix B.

The fuel assembly structural materials are assumed to be compacted to $70 \%$ of their theoretical density, resulting in final structural material volumes of $2.29 \mathrm{ft}^{3}$ per metric ton of PWR fuel, $2.92 \mathrm{ft}^{3}$ per metric ton of BWR fuel, and $8.7 \mathrm{ft}^{3}$ per metric ton of LMFBR fuel. The compacted structural materials are packaged in canisters $10 \mathrm{irl}$. in diameter by $10 \mathrm{ft}$ long, each of which contains $3.53 \mathrm{ft}^{3}$ of waste. After a storage period of five years at the reprocessing plants, the waste is shipped by rail to a repository in casks containing 27 canisters of LWR cladding or 36 canisters of LMFBR cladding.

The properties (volume, mass, radioactivity, thermal power, and toxicity) of the cladding wastes accumulated at one or more federal repositories are presented in Table 4.21. Many of the assumptions used to construct this table are based on an earlier study of cladding wastes. 25

The accumulated mass, radioactivity, thermal power, and ingestion toxicity of the cladding wastes as a function of time following the year 2005 are summarized in Tables 4.22 through 4.25 for neutroninduced radionuclides, Tables 4.26 through 4.29 for associated actinides, and Tables 4.30 through 4.33 for associated fission products.

\subsection{Intermediate-Level TRU Wastes}

Intermediate-level transuranic (TRU) wastes are defined as those solid llateridls, uther than hlgh-level and cladding wastes, which contain long-lived alpha activities qreater than $10 \mathrm{nCi} / \mathrm{g}$ and have gamma radiation levels sufficient to require biological shielding and remote handling techniques (typical surface dose rates lie between 10 and $1000 \mathrm{mrem} / \mathrm{hr}$ ). The criterion of $10 \mathrm{nCi} / \mathrm{g}$ is adopted as a lower activity density for low-level TRU wastes because this corresponds to 
Table 4.21. Projected annual accumulation of clading waste at a federal repository

\begin{tabular}{|c|c|c|c|c|c|}
\hline ear & $\begin{array}{l}\text { Volume } \\
\text { (Thousands } \\
\text { of } \mathbf{m}^{3} \text { ) }\end{array}$ & $\begin{array}{l}\text { Annual A } \\
\text { Actinide } \\
\text { पass } \\
\text { (MT) }\end{array}$ & $\begin{array}{c}\text { ddition } \\
\text { Radio- } \\
\text { activity } \\
\text { (HCi) }\end{array}$ & $\begin{array}{l}\text { **** } \\
\text { Thermal } \\
\text { Power } \\
\text { (Hi) }\end{array}$ & $\begin{array}{c}\text { Annual } \\
\text { Number } \\
\text { of } \\
\text { Shipments }\end{array}$ \\
\hline $\begin{array}{l}1986 \\
1987 \\
1988 \\
1989 \\
1990 \\
1991 \\
1992 \\
1993 \\
1994 \\
1995 \\
1996 \\
1997 \\
1998 \\
1999 \\
2000 \\
2001 \\
2002 \\
2003 \\
2004 \\
2005\end{array}$ & $\begin{array}{l}0.04 \\
0.11 \\
0.11 \\
0.11 \\
0.14 \\
0.22 \\
0.22 \\
0.25 \\
0.32 \\
0.36 \\
0.43 \\
0.47 \\
0.54 \\
0.58 \\
0.65 \\
0.69 \\
0.80 \\
0.88 \\
0.81 \\
0.78\end{array}$ & $\begin{array}{l}0.24 \\
0.72 \\
0.72 \\
0.72 \\
0.97 \\
1.45 \\
1.45 \\
1.69 \\
2.18 \\
2.42 \\
2.91 \\
3.15 \\
3.63 \\
3.87 \\
4.40 \\
4.65 \\
5.34 \\
5.84 \\
5.31 \\
4.95\end{array}$ & $\begin{array}{r}3.3 \\
10.2 \\
10.9 \\
12.0 \\
16.6 \\
24.4 \\
23.7 \\
27.2 \\
34.7 \\
38.8 \\
47.3 \\
52.2 \\
60.8 \\
64.9 \\
73.5 \\
77.4 \\
89.9 \\
100.3 \\
91.9 \\
85.9\end{array}$ & $\begin{array}{l}0.015 \\
0.048 \\
0.050 \\
0.052 \\
0.071 \\
0.102 \\
0.099 \\
0.114 \\
0.145 \\
0.163 \\
0.199 \\
0.220 \\
0.257 \\
0.274 \\
0.311 \\
0.328 \\
0.380 \\
0.423 \\
0.386 \\
0.359\end{array}$ & $\begin{array}{r}30 \\
95 \\
81 \\
75 \\
96 \\
147 \\
146 \\
168 \\
219 \\
247 \\
297 \\
321 \\
370 \\
394 \\
448 \\
474 \\
544 \\
596 \\
543 \\
509\end{array}$ \\
\hline
\end{tabular}

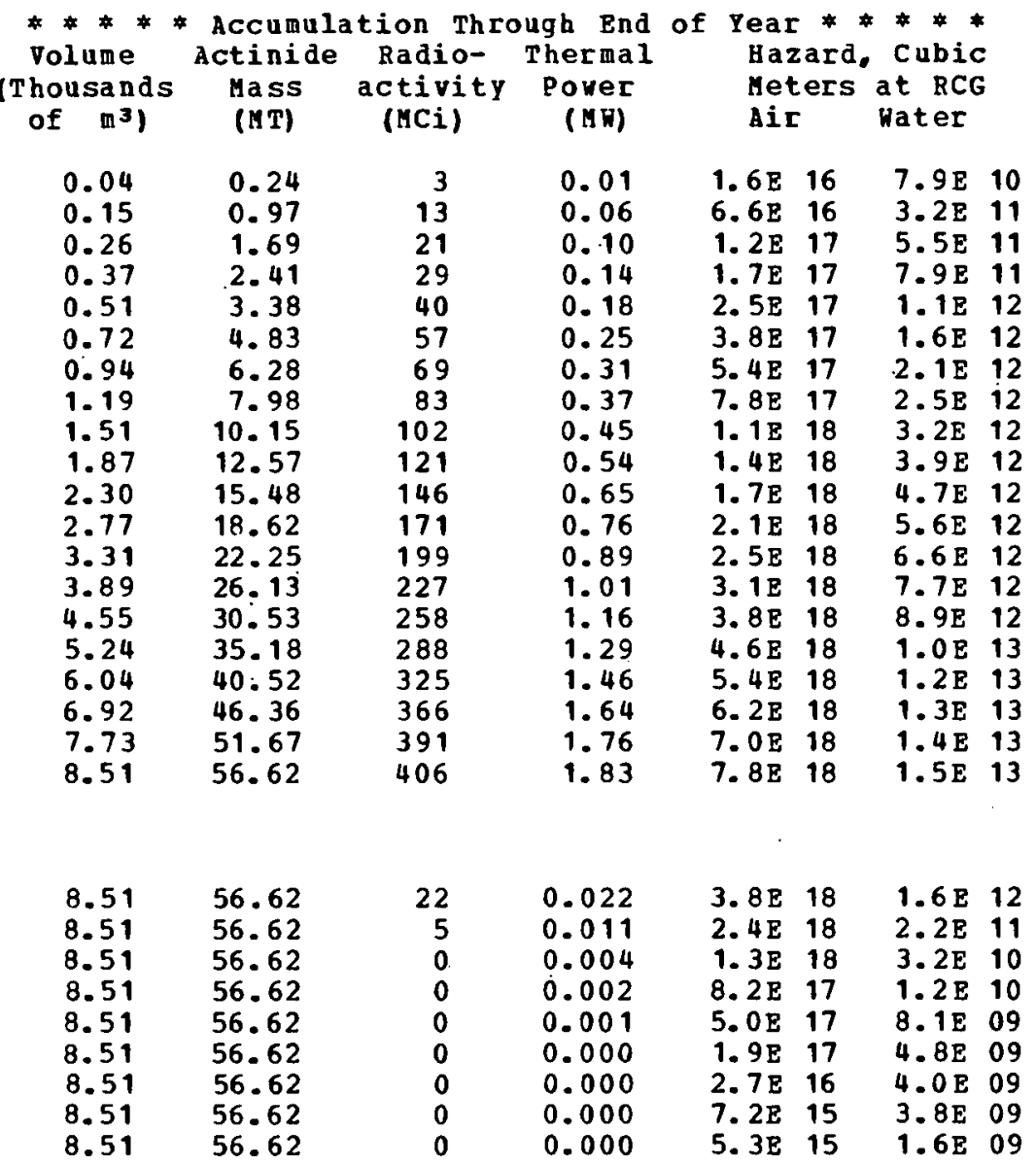


Table 4.22. Grams of accumulated structural materials and impurity elements in the cladding wastes at a federal repository in the year 2005 as a function of age

\begin{tabular}{|c|c|c|c|c|c|c|c|c|c|c|c|c|c|c|c|c|}
\hline \multirow[b]{2}{*}{$\begin{array}{l}\text { HE } \\
\text { LI } \\
B E\end{array}$} & \multicolumn{2}{|c|}{$\begin{array}{l}\text { Year } \\
2005\end{array}$} & 100 & \multicolumn{5}{|c|}{ 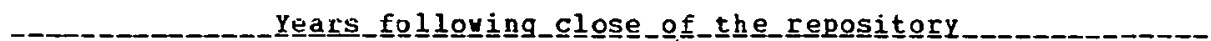 } & 3000 & & \multicolumn{2}{|c|}{10,000} & \multicolumn{2}{|c|}{100.000} & \multicolumn{2}{|c|}{$1,000,000$} \\
\hline & $\begin{array}{l}4 \mathrm{E} \\
0 \mathrm{E} \\
1 \mathrm{E}\end{array}$ & $\begin{array}{l}03 \\
03 \\
01\end{array}$ & $\begin{array}{l}2.34 \mathrm{E} \\
1.80 \mathrm{E} \\
1.51 \mathrm{E}\end{array}$ & $\begin{array}{l}03 \\
03 \\
01\end{array}$ & $\begin{array}{l}4 E \\
0 E \\
1 E\end{array}$ & $\begin{array}{l}03 \\
03 \\
01\end{array}$ & $\begin{array}{l}2.34 \mathrm{E} \\
1.80 \mathrm{E} \\
1.51 \mathrm{E}\end{array}$ & $\begin{array}{l}03 \\
03 \\
01\end{array}$ & $\begin{array}{l}2.34 \mathrm{E} \\
1.80 \mathrm{E} \\
1.51 \mathrm{E}\end{array}$ & $\begin{array}{l}03 \\
03 \\
01\end{array}$ & $\begin{array}{l}2.34 \mathrm{E} \\
1.80 \mathrm{P} \\
1.51 \mathrm{E}\end{array}$ & $\begin{array}{l}03 \\
03 \\
01\end{array}$ & $\begin{array}{l}2.34 \mathrm{E} \\
1.80 \mathrm{E} \\
1.51 \mathrm{E}\end{array}$ & $\begin{array}{l}03 \\
03 \\
01\end{array}$ & $\begin{array}{l}2.34 \mathrm{E} \\
1.80 \mathrm{E} \\
1.48 \mathrm{E}\end{array}$ & $\begin{array}{l}03 \\
01\end{array}$ \\
\hline $\mathrm{B}$ & $6 \mathrm{E}$ & 04 & $16 \mathrm{E}$ & 04 & $1.16 \mathrm{E}$ & 04 & 1. $16 \mathrm{E}$ & 04 & 1. $16 \mathrm{E}$ & 04 & $6 ?$ & 04 & $.16 \mathrm{E}$ & 04 & $\mathbf{E}$ & 04 \\
\hline C & $98 \mathrm{~F}$ & 06 & $98 \mathrm{E}$ & 06 & $8.98 \mathrm{P}$ & 06 & 9. $98 \mathrm{E}$ & 06 & $8.98 \mathrm{E}$ & 06 & $8.98 \mathrm{E}$ & 06 & $8.98 \mathrm{E}$ & 06 & $E$ & 06 \\
\hline $\mathbf{N}$ & $26 \mathrm{E}$ & 05 & & 05 & $6 \mathrm{E}$ & 05 & $26 \mathrm{E}$ & 05 & $7 \mathrm{E}$ & 0 & $8 \mathrm{E}$ & 05 & & 05 & & 05 \\
\hline 0 & $2 \mathrm{E}$ & 07 & $2 E$ & 0.1 & $62 \mathrm{E}$ & 07 & $62 \mathrm{E}$ & 07 & & 0 & $2 \mathrm{E}$ & 07 & $2 \mathrm{E}$ & 07 & & 7 \\
\hline $\mathrm{F}$ & $1.77 \mathrm{E}$ & 01 & $1.77 \mathrm{E}$ & 01 & $1.77 \mathrm{E}$ & 01 & $1.77 \mathrm{E}$ & 01 & $1.77 \mathrm{E}$ & 01 & $.77 \mathrm{E}$ & 01 & $1.77 \mathrm{E}$ & 01 & $E$ & 01 \\
\hline $\mathrm{NE}$ & $8.03 \mathrm{E}$ & 00 & $8.04 \mathrm{E}$ & 00 & $8.04 \mathrm{E}$ & 00 & $8.04 \mathrm{E}$ & 00 & $8.04 \mathrm{E}$ & 00 & $8.04 \mathrm{~F}$ & 00 & $8.04 \mathrm{E}$ & 00 & $4 E$ & 0 \\
\hline $\mathbf{N} \dot{A}$ & $6.46 \mathrm{E}$ & 05 & $6.46 \mathrm{E}$ & 05 & $6.46 \mathrm{E}$ & 05 & $6.46 \mathrm{E}$ & 05 & $6.46 \mathrm{E}$ & 05 & $6.46 \mathrm{E}$ & 05 & $6.46 \mathrm{E}$ & 05 & $6.46 \mathrm{E}$ & 05 \\
\hline MG & $1 \mathrm{E}$ & 02 & $4.01 \mathrm{E}$ & 02 & $4.01 \mathrm{E}$ & 02 & $4.01 \mathrm{E}$ & 02 & $4.01 \mathrm{E}$ & 02 & $4.01 \mathrm{E}$ & 02 & $4.01 \mathrm{E}$ & 02 & 4.015 & 02 \\
\hline$A L$ & $8 \mathrm{E}$ & 06 & $8.58 \mathrm{E}$ & 06 & $8.58 \mathrm{E}$ & 06 & $8.58 \mathrm{E}$ & 06 & $8.58 \mathrm{E}$ & 06 & $8.58 \mathrm{E}$ & 06 & $8.58 \mathrm{E}$ & 06 & 8. & 06 \\
\hline$S I$ & $9 \mathrm{E}$ & 07 & 3. & 07 & $3.59 \mathrm{E}$ & 07 & $3.59 \mathrm{E}$ & 07 & $3.59 \mathrm{E}$ & 07 & 3.5 & 07 & 3. & 07 & $\mathrm{~F}$ & 07. \\
\hline P & $5 \mathrm{E}$ & 07 & & 07 & $1.95 \mathrm{E}$ & 07 & $.1 .95 \mathrm{E}$ & 07 & $1.95 \mathrm{E}$ & 07 & $5 \mathrm{E}$ & 0 & & 07 & & 07 \\
\hline $\mathrm{S}$ & $56 \mathrm{E}$ & 06 & $1.56 \mathrm{E}$ & 06 & $1.56 \mathrm{E}$ & 06 & $1.56 \mathrm{E}$ & 06 & $1.56 E$ & 06 & $.56 \mathrm{E}$ & 0 & $56 \mathrm{E}$ & 06 & 5 & 06 \\
\hline CL & $13 \mathrm{E}$ & 01 & $2.13 E$ & 01 & $2.13 E$ & 01 & $2.13 \mathrm{E}$ & 01 & $2.13 \mathrm{E}$ & 0 & $2.13 F$ & 01 & $2.12 \mathrm{E}$ & 01 & 2. & 01 \\
\hline 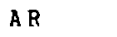 & 1 & -04 & 2. & -0 & $5.34 \%-$ & .04 & $1.51 \mathrm{E}$ & -03 & $4.29 \mathrm{E}-$ & .03 & 1. $39 \mathrm{E}-$ & -02 & 1.2 & -01 & & \\
\hline K & 2. & -06 & & -06 & $2.43 \mathrm{E}-$ & 06 & $?$ & -06 & $2.44 \mathrm{E}-$ & 06 & $2.44 \mathrm{E}-$ & -06 & $\mathrm{E}$ & -06 & & 06 \\
\hline CA & $23 \mathrm{E}$ & 01 & 3. & .01 & $3.23 \mathrm{E}$ & 01 & $3.23 \mathrm{E}$ & 01 & $3.23 \mathrm{E}$ & 01 & $3.23 E$ & 01 & $.23 \mathrm{E}$ & 01 & $3.23 \mathrm{E}$ & 01 \\
\hline C & $5.25 \mathrm{E}-$ & -01 & 5 & -0 & $5.25 \mathrm{E}-$ & 01 & 5.2 & -01 & 5.2 & 01 & 5.2 & -01 & E & -01 & 5. & -01 \\
\hline TI & $1.22 \mathrm{E}$ & 07 & 1. & 07 & 1. $22 \mathrm{E}$ & 07 & 1.22 & 07 & 1. 2 & 07 & 1.2 & 07 & $\mathrm{~F}$ & 07 & 1. & 07 \\
\hline V & $1.10 \mathrm{E}$ & 06 & $1.10 \mathrm{E}$ & 06 & 1. $10 \mathrm{E}$ & 06 & $1.10 \mathrm{E}$ & 06 & $1.10 \mathrm{~g}$ & 06 & 1. & 0 & $1.10 \mathrm{E}$ & 06 & 1. & 06 \\
\hline$C P$ & $1.20 \mathrm{E}$ & 09 & & 09 & $1.20 \mathrm{E}$ & 09 & $1.20 \mathrm{E}$ & 09 & $1.20 \mathrm{E}$ & 0 & $1.20 \mathrm{E}$ & 09 & $1.20 \mathrm{E}$ & 09 & $1.20^{\circ} \mathrm{E}$ & 09 \\
\hline$M N$ & $E$ & 07 & $6.30 \mathrm{E}$ & 0 & $6.30 \mathrm{E}$ & 07 & 6.3 & 07 & 6.3 & 07 & 6.3 & 07 & $E$ & 07 & 6.3 & 07 \\
\hline$F F$ & $70 E$ & 09 & $3.70 \mathrm{E}$ & 09 & $3.70 \mathrm{E}$ & $0 \%$ & $3.70 \mathrm{E}$ & 09 & 3.705 & 09 & 705 & 09 & $70 \mathrm{E}$ & 09 & 3.7 & 09 \\
\hline $\mathrm{CO}$ & $50 \mathrm{E}$ & 06 & $2.45 \mathrm{E}$ & 06 & $2.46 \mathrm{~F}$ & 06 & $2.48 \mathrm{E}$ & 06 & $2.54 \mathrm{E}$ & 06 & $2.76 \mathrm{~F}$ & 06 & $66 \mathrm{E}$ & 06 & 6.2 & 06 \\
\hline NI & $.03 \mathrm{E}$ & 09 & 1.03 & 0 & & 0.9 & & 09 & $E$ & 0 & 1. & 09 & & 09 & $2 \mathrm{E}$. & 09 \\
\hline $\mathrm{CU}$ & $E$ & 06 & 2. & 06 & 2. & 06 & 2 & 06 & 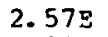 & 06 & 2. & 06 & $E$ & 06 & $F$ & nk \\
\hline $\mathrm{ZN}$ & & $\dot{0}$ & $9.58 \mathrm{E}$ & 03 & $3 \mathrm{E}$ & 03 & $\mathrm{E}$ & 03 & $E$ & 03 & & 03 & & 03 & & 03 \\
\hline SR & $6 \mathrm{E}$ & 04 & $2.56 \mathrm{E}$ & 04 & $2.56 \mathrm{E}$ & 04 & $56 \mathrm{~B}$ & 04 & $2.56 \mathrm{E}$ & 04 & $6 \mathrm{E}$ & 04 & $56 \mathrm{E}$ & 04 & $2.56 \mathrm{E}$ & 04 \\
\hline$Y$ & & -04 & & -0 & & 08 & & -15 & $15-$ & 3 & & & & & & \\
\hline $2 \mathrm{R}$ & $6 \mathrm{E}$ & 10 & $2.76 \mathrm{E}$ & 10 & 2.7 & 10 & $\mathrm{E}$ & 10 & $2.76 \mathrm{E}$ & 10 & 2 & 10 & $6 \mathrm{E}$ & 10 & $\mathrm{E}$ & 10 \\
\hline NB & $2 \mathrm{E}$ & 07 & $4.82 \mathrm{E}$ & 97 & $4.82 \mathrm{E}$ & 07 & $82 \mathrm{E}$ & 07 & $4.82 \mathrm{E}$ & 07 & $4.82 \mathrm{E}$ & 07 & $4.84 \mathrm{E}$ & 07 & 4. & 07 \\
\hline 190 & $12 \mathrm{E}$ & 07 & $3.42 \mathrm{E}$ & 0 & $3.42 \mathrm{E}$ & 07 & $42 \mathrm{E}$ & 07 & $3.42 \mathrm{E}$ & 0 & $3.42 \mathrm{E}$ & 07 & $2 E$ & 07 & 3. & 07 \\
\hline TC & $3 E$ & 04 & $5.22 \mathrm{E}$ & 04 & $5.22 \mathrm{E}$ & 04 & & 04 & & 04 & 5. & 04 & $5 \mathrm{E}$ & 04 & 1. & 03 \\
\hline RU & & 04 & $7 \mathrm{E}$ & 04 & 1. $77 \mathrm{E}$ & 04 & & 04 & $1.77 \mathrm{E}$ & 04 & $7 \mathrm{E}$ & 04 & $7 E$ & 04 & $1.77 \mathrm{E}$ & 04 \\
\hline$C D$ & $4 \mathrm{E}-$ & -02 & $2.43 \mathrm{E}-$ & .02 & $2.43 \mathrm{E}$ & 02 & $2.43 \mathrm{~K}=$ & .02 & $2.43 E-$ & 02 & $2.43 \mathrm{E}-$ & -02 & $2.43 \mathrm{E}$ & .02 & $2.43 \mathrm{E}-$ & -02 \\
\hline $\mathrm{SN}$ & $31 \mathrm{E}$ & 08 & $4.31 \mathrm{E}$ & 08 & $4.31 \mathrm{E}$ & 08 & $4.31 \mathrm{E}$ & 08 & $4.31 \mathrm{E}$ & 08 & $4.31 \mathrm{E}$ & 08 & $4.31 \mathrm{E}$ & 08 & $4.31 \mathrm{E}$ & 08 \\
\hline SB & & 07 & $2.49 E$ & 07 & 2.495 & 07 & & 07 & $2.49 \mathrm{~B}$ & 07 & $2.49 \mathrm{E}$ & 07 & 2. & 07 & $2.49 \mathrm{E}$ & 07 \\
\hline $\mathrm{TE}$ & $91 \mathrm{E}$ & 06 & $4.05 \mathrm{E}$ & 06 & $4.05 \mathrm{E}$ & 06 & $4.05 \mathrm{E}$ & 06 & $4.05 \mathrm{E}$ & 06 & $4.05 \mathrm{E}$ & 06 & $4.05 \mathrm{E}$ & 06 & $4.05 \mathrm{E}$ & 06 \\
\hline TA & $2 \mathrm{E}$ & 00 & $6.52 \mathrm{~F}$ & 00 & $6.52 \mathrm{E}$ & 00 & $6.52 \mathrm{E}$ & 00 & $6.52 \mathrm{E}$ & 00 & $6.52 \mathrm{P}$ & 00 & $0.52 \mathrm{E}$ & 00 & $6.52 \mathrm{E}$ & 00 \\
\hline . & & 05 & $6 \mathrm{E}$ & 0 & & 05 & & 0 & & 05 & $2.96 \mathrm{E}$ & 05 & & 05 & & ככנ \\
\hline TOT & $4 E$ & 10 & $3.44 \mathrm{E}$ & 10 & $3.44 \mathrm{E}$ & 10 & $3.44 \mathrm{E}$ & 10 & $3.44 E$ & 10 & $3.44 \mathrm{~F}$ & 10 & 48 & 10 & $3.44 \mathrm{E}$ & 10 \\
\hline
\end{tabular}


Table 4.23. Curies of accrmulated. structural materials and impurity isotopes in the cladiling wastes at a federal repository

in the year 2005 as a function of age

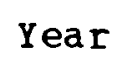
2005

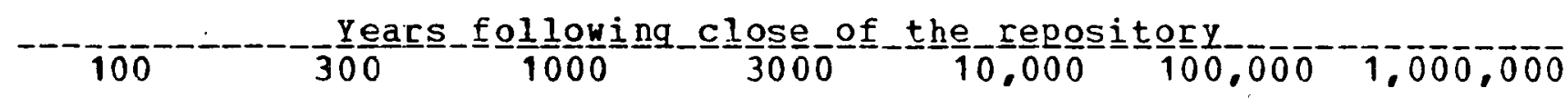

C 14 $M N 54$ FE 55 CO 60 NI 59 NI 63 ZR 93 NB $93 M$ NB 94 MO 93 TC 99 SN $121 \mathrm{M}$ SB 125 TE125M TOTAL
1. 15E 04 4. 10E 05 $6.48 \mathrm{E} \quad 07$ 6. $34 \mathrm{E} 07$ 2. $90 \mathrm{E} 05$ 4. $13 \mathrm{E} \quad 07$ 1. $13 \mathrm{E} 04$ $6.44 \mathrm{E} 03$ 1. $74 \mathrm{E} \quad 01$ $2.97 \mathrm{E} \quad 03$ 8. $99 \mathrm{E} \quad 02$ $3.71 \mathrm{E} 04$ 1.51E 08 $6.26 \mathrm{E} \quad 07$ 3. $84 \mathrm{E} 08$

\begin{abstract}
$1.13 \mathrm{E} 04$
\end{abstract} 1. $71 \mathrm{E}-04$ 1.20E 02 2. $90 \mathrm{E} 05$ 1.95E 07 1. $13 \mathrm{E} \quad 04$ 1. 38 E 04 1.745 01 2. $95 E 03$ 8. $99 \mathrm{E} \quad 02$ 1. $49 \mathrm{E} \quad 04$ $1.07 E-03$ 4. $44 E-04$

$\begin{array}{llllllll}1.98 \mathrm{E} & 07 & 4.64 \mathrm{E} & 06 & 3.48 \mathrm{E} & 05 & 3.18 \mathrm{E} & 05\end{array}$

4. $29 \mathrm{E}-10$

2.89E O5 2.87E $05 \quad 2.82 \mathrm{E} \quad 05$

4. $31 \mathrm{E} 06$

$2.21 \mathrm{E} 046.30 \mathrm{E}-03$

$\begin{array}{llllll}1.38 \mathrm{E} & 04 & 1.13 \mathrm{E} & 04 & 1.13 \mathrm{E} & 04 \\ 36 & 04 & 1.33 \mathrm{E} & 04\end{array}$

$\begin{array}{lllllll}1.74 \mathrm{E} & 01 & 1.74 \mathrm{E} & 01 & 1.74 \mathrm{E} & 01\end{array}$

$2.91 \mathrm{E} \quad 03 \quad 2.75 \mathrm{E} \quad 03 \quad 2.36 \mathrm{E} \quad 03$

$8.98 \mathrm{E} \quad 02 \quad 8.96 \mathrm{E} \quad 02 \quad 8.90 \mathrm{E} \quad 02$

$2.40 \mathrm{E} \quad 03 \quad 3.98 \mathrm{E} \quad 00 \quad 4.76 \mathrm{E}-08$
3. $43 \mathrm{~F}, 0.3$

6. $40 \mathrm{~s}-02$

$2.66 E 05$

1. $12 \mathrm{~F} 04$

$1.24 \mathrm{E} 04$

$1.74 \mathrm{E} 01$

1. $38 \mathrm{E} \quad 03$

$8.70 \mathrm{E} 02$

1. $22 \mathrm{E} \quad 05$

$5.03 \mathrm{E} 01$

$\begin{array}{llll}1.08 \mathrm{E} & 04 & 7.11 \mathrm{E} & 03\end{array}$

$1.08 \mathrm{E} \quad 04 \quad 7.11 \mathrm{E} 03$

$1.73 \mathrm{E} \quad 01 \quad 1.68 \mathrm{E} \quad 01$

$1.34 \mathrm{~F} 00$

$6.46 \mathrm{E} \quad 02 \quad 3.31 \mathrm{E} \quad 01$ 
Table 4.24. Natts of accumulated structural materials and impurity isotopes in the cladding wastes at a federal repository

in the year 2005 as a function of age

Year 2005

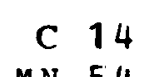

MN 5,4

FE 5.5

CO 60

NI $\in 3$

ZR 93

NB $93 \mathrm{M}$

NB $\subseteq 4$

MO 93

TC 99

SN $121 \mathrm{M}$

SB1 25

TE125M

TOTAI

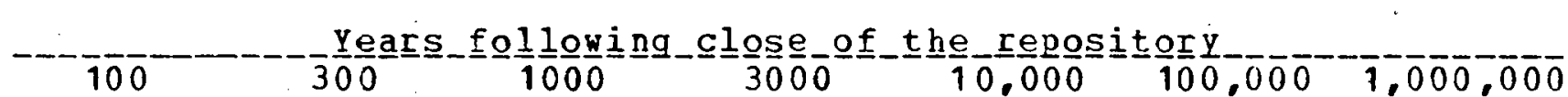

$3.40 \mathrm{~F} 00$

$3.32 \mathrm{~F} \quad 03$

$8.46 \mathrm{E} \quad 04$

$9.91 \mathrm{E} 05$

$6.62 \mathrm{E} 03$

$1.34 \mathrm{~F} 00$

2. $25 \mathrm{E} 00$

2. $03 E-01$

$7.40 \mathrm{~F} \quad 0 \mathrm{CO}$

$1.55 \mathrm{E} O C$

$3.8 \mathrm{E} \quad 01$

$6.12 \mathrm{~F} 05$

$5.38 \mathrm{E} 04$

$3.36 \mathrm{E} 00$

$3.28 \mathrm{E} \quad 00^{\circ}$

$3.02 \mathrm{E} 00$

$2.37 \mathrm{E} 00$

$1.02 \mathrm{E} 00$

1. $90 \mathrm{E}-05$

2. $23 \mathrm{E}-07$

1.87 F 00

3.11903

$6.71 \mathrm{E}-12$

$1.34 \mathrm{E} \quad 00$

$6.90 \mathrm{E} \quad 02$

$1.34 \mathrm{E} \quad 00$

$3.53 \mathrm{E} 00$

$1.01 \mathrm{E}-06$

$2.03 \mathrm{E}-01 \quad 2.03 \mathrm{E}-01 \quad 2.03 \mathrm{E}-01$

$2.03 E-01$

$5.88 \mathrm{E} 00$

$1.53 \mathrm{E} \quad 00$

$1.54 \mathrm{E} \quad 00$

$1.54 \mathrm{E} 00$

$1.54=00$

$1.56 \mathrm{E} \quad 01$

4. 34E-06

$2.52 E 00 \quad 4.17 E-03 \quad 4.09 F-11$

1. $33 \mathrm{E} 00$

4. $34 \mathrm{E} 00$

2. $03 \mathrm{E}-01$

3. $43 \mathrm{E} 00$

1. $50 \mathrm{E} 00$

$1.28 \mathrm{E} 00$

3. $77 \mathrm{E} \quad 00$

2. $025-01$

3. $35 \mathrm{~F}-03$

3. $81.5-07$

$1.75 \mathrm{E} 06$

$\begin{array}{llllllllll}3.15 E & 03 & -7.11 F & 02 & 2.13 E & 01 & 1.60 F & 01\end{array}$

$1.18 F 01$

$6.37 F .00 \quad 3.59 \mathrm{~F} 00$

$8.43 F-01$

$2.49 \mathrm{E} 00$

$1.96 \mathrm{E}-01$

$5.70 \mathrm{E}-02$ 
Table 4.25. Ingestion toxicity ( $\mathrm{m}^{3}$ water) of accumulated structural materials and impurity isotopes in the cladding wastes at a federal

repository in the year 2005 as a function of age

Year

2005

C 14

MN 54

FE 55

$\mathrm{CO} 60$

NI 59

NI 63

$\mathrm{ZR} 93$

$\mathrm{NB} 93 \mathrm{M}$

NB 94

MO 93

TC 99

SN $121 \mathrm{M}$

SB125

TE125M

TOTAL
$1.44 \mathrm{E} .07$

4. 10E 09

$8.11 \mathrm{E} 10$

2. 11E 12

$1.45 \mathrm{E} 09$

1. $38 \mathrm{E} \quad 12$

$1.41 \mathrm{E} 07$

$1.61 \mathrm{E} 07$

$1.74 \mathrm{E} 01$

$2.97 \mathrm{E} 03$

4. $49 \mathrm{E} 06$

$3.71 \mathrm{E} \quad 04$

$1.51 \mathrm{E} \quad 12$

$6.26 \mathrm{E} \quad 11$

$5.71 \mathrm{E} \quad 12$
$-100$

$1 \overline{0} \overline{0}$

$100,0 \overline{0} \overline{1}, \overline{0} 0 \overline{0}, \overline{0} \overline{0} \overline{0}$

$1.42 E \quad 07$

$1.38 \mathrm{E} 07$

$1.27 \mathrm{E} 07 \quad 9.99 \mathrm{E} 06$

4. $28 E 06$

8. $01 \mathrm{E} 01$

2. $14 \mathrm{E}-01$

4.00E $06 \quad 1.43 E-05$

$1.45 \mathrm{E} \quad 09 \quad 1.45 \mathrm{E} \quad 09$

$6.49 \mathrm{E} \quad 11$

$1.41 \mathrm{E} \quad 07$

$3.44 \mathrm{E} \quad 07$

$1.44 \mathrm{E} 11$

$1.44 \mathrm{E} \quad 09 \quad 1.41 \mathrm{E} \quad 09$

$\begin{array}{llllll}1.41 \mathrm{E} & 07 & 1.41 \mathrm{E} & 07 & 1.41 \mathrm{E} & 07\end{array}$

$\begin{array}{lllllll}3.44 \mathrm{E} & \mathrm{C} 7 & 3.41 \mathrm{E} & 07 & 3.32 \mathrm{E} & 07\end{array}$

$\begin{array}{llllllll}1.74 \mathrm{E} & 01 & 1.74 \mathrm{E} & \mathrm{C} 1 & 1.74 \mathrm{E} & 01 & 1.74 \mathrm{E} & 01\end{array}$

$2.95 \mathrm{E} 03$

$2.91 \mathrm{E} C 3 \quad 2.75 \mathrm{E} 03$

$2.36 \mathrm{~F} 03$

$4.49 \mathrm{E} 06$

$4.49 \mathrm{E} C 6 \quad 4.48 \mathrm{E} \quad 06$

$1.49 \mathrm{E} 04$

$2.40 \mathrm{EC} 33.98 \mathrm{E} 00 \quad 4.76 \mathrm{E}-08$

1. $33 \mathrm{E} 09$

$6.09 \mathrm{E} 08$

$2.51 \mathrm{E} 05$

$\stackrel{\infty}{\infty}$

$1.07 \mathrm{E} \quad 01$

4. $44 \mathrm{E} \quad 00$

$6.50 \mathrm{E} \quad 11$

$\begin{array}{llllll}1.45 \mathrm{E} & 11 & 2.24 \mathrm{E} & 09 & 1.47 \mathrm{E} & 09\end{array}$
$1.41 \mathrm{E} 07$

1. $74 \mathrm{E} 01$

1. $38 \mathrm{E} 03$

4. $35 \mathrm{E} 06$

1. $35 \mathrm{E} \quad 07$

2. 70E 07

$1.34 \mathrm{E} 00$

$3.23 \mathrm{~F} 06$

$8.89 \mathrm{E} 05$

1. $78 \mathrm{E} 07$

$1.66 \mathrm{E} 05$

1. $38 \mathrm{E} 09$

$6.53 \mathrm{E} \quad 08 \quad 2.71 \mathrm{E} \quad 07$
1. $73 \mathrm{E} 01$

1. $68 \mathrm{E} 01$ 
Table 4.26. Grams of accumulated heavy elements in the cladding wastes at a federal repository

in the year 2005 as a function of age

\begin{tabular}{|c|c|c|c|c|c|c|c|c|}
\hline & Year & & & 으 & $=$ 으 & 11 & & \\
\hline & 5 & 100 & 300 & 1000 & 3000 & 10,000 & 100.000 & 1.000 .000 \\
\hline $\mathrm{HE}$ & $.58 \mathrm{E} \quad 01$ & $96 \mathrm{E} \quad 02$ & $07 \mathrm{E} \quad 03$ & $2.13 \mathrm{E} \quad 03$ & .30503 & $.47 \mathrm{E} 03$ & $2 \mathrm{E} \quad 04$ & $1.84 \mathrm{E}$ \\
\hline I & -00 & $E-10$ & $E-10$ & $E-11$ & -10 & $E-10$ & $F-0$ & $E-08$ \\
\hline 5 & 03 & -0 & $:-0$ & -02 & 1 & E 01 & $\overrightarrow{\mathrm{a}}$ & 04 \\
\hline$I$ & 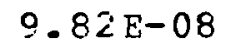 & 6 & $E-05$ & $E-03$ & 4.4 & E 00 & $\mathrm{E} \quad 02$ & $E 04$ \\
\hline ? & -10 & $?$ & $5 E-06$ & $8-05$ & $2 . \circ$ & $E-03$ & $7 z-02$ & $E-0$ \\
\hline $\mathbf{T}$ & & -17 & $8:-16$ & -15 & -14 & 7.9 & $E-$ & $5-1$ \\
\hline $2 \mathrm{~N}$ & & $-c$ & $E-0$ & -06 & .06 & & $E-$ & $E-0$ \\
\hline $\mathrm{f}$ & & - & $E-$ & & 10 & & $\mathrm{~F}$ & -07 \\
\hline 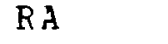 & & -1 & $\pi-1$ & & & & & 01 \\
\hline C & & 3 & $E-($ & 24 & -04 & -03 & 2 & -02 \\
\hline ए曰 & & 4 & 1 & 01 & 02 & E 02 & $\mathrm{E}$ & E 04 \\
\hline PA & -02 & -0 & $E-01$ & $E-01$ & 11E 00 & E 00 & E 01 & E 01 \\
\hline 0 & 07 & $5.12 \mathrm{E} \quad 07$ & $2 \mathrm{E} \quad 07$ & E 07 & $5.12 \mathrm{E} 07$ & $5.13 \mathrm{~F} 07$ & E 07 & F 07 \\
\hline N & 04 & $7 \mathrm{~F}: 04$ & $6.97 \mathrm{E} \quad 04$ & E 05 & $1.33 \mathrm{E} 05$ & 1. $33 E 0.5$ & $O E 05$ & E 04 \\
\hline PU & E 05 & $5.62 \mathrm{E} \quad 05$ & $5.53 \mathrm{E} 05$ & E 05 & $4.89 \mathrm{E} \quad 05$ & $3.71 \mathrm{E} 05$ & 3504 & 503 \\
\hline 16 & $E 04$ & E 05 & $7.80 E \quad 04$ & E 04 & 1.12504 & $5.46 \mathrm{E} 03$ & E 00 & $5 E-04$ \\
\hline $\mathrm{M}$ & 03 & $8 \mathrm{E} \quad 02$ & $8.51 \mathrm{E} \quad 02$ & E 02 & $6.69 \mathrm{~F} \quad 02$ & 3.62502 & $3 \mathrm{E} \quad 00$ & $7 \mathrm{E} \quad 00$ \\
\hline $\mathrm{BF}$ & $8-06$ & $7 F-18$ & $0 E-18$ & $E-18$ & 7. $99 E-18$ & $6.04 \mathrm{E}-18$ & $3 F-19$ & $E-35$ \\
\hline & -03 & $7 E-03$ & $9 \mathrm{E}-0$ & $1 E-04$ & $1.98 E-05$ & $6.42 E-08$ & $6 z-15$ & $60 E-30$ \\
\hline & & & & & & & & \\
\hline & $9 \mathrm{E} \quad 07$ & 07 & $19 E 07$ & 19E 07 & 9E 07 & . $19 E \quad 07$ & . $1 \subseteq E \quad 07$ & 108 \\
\hline
\end{tabular}


Table 4.27. Curies of accumulated heavy isotopes

in the cladding wastes at a federal repository in the year 2005 as a function of age

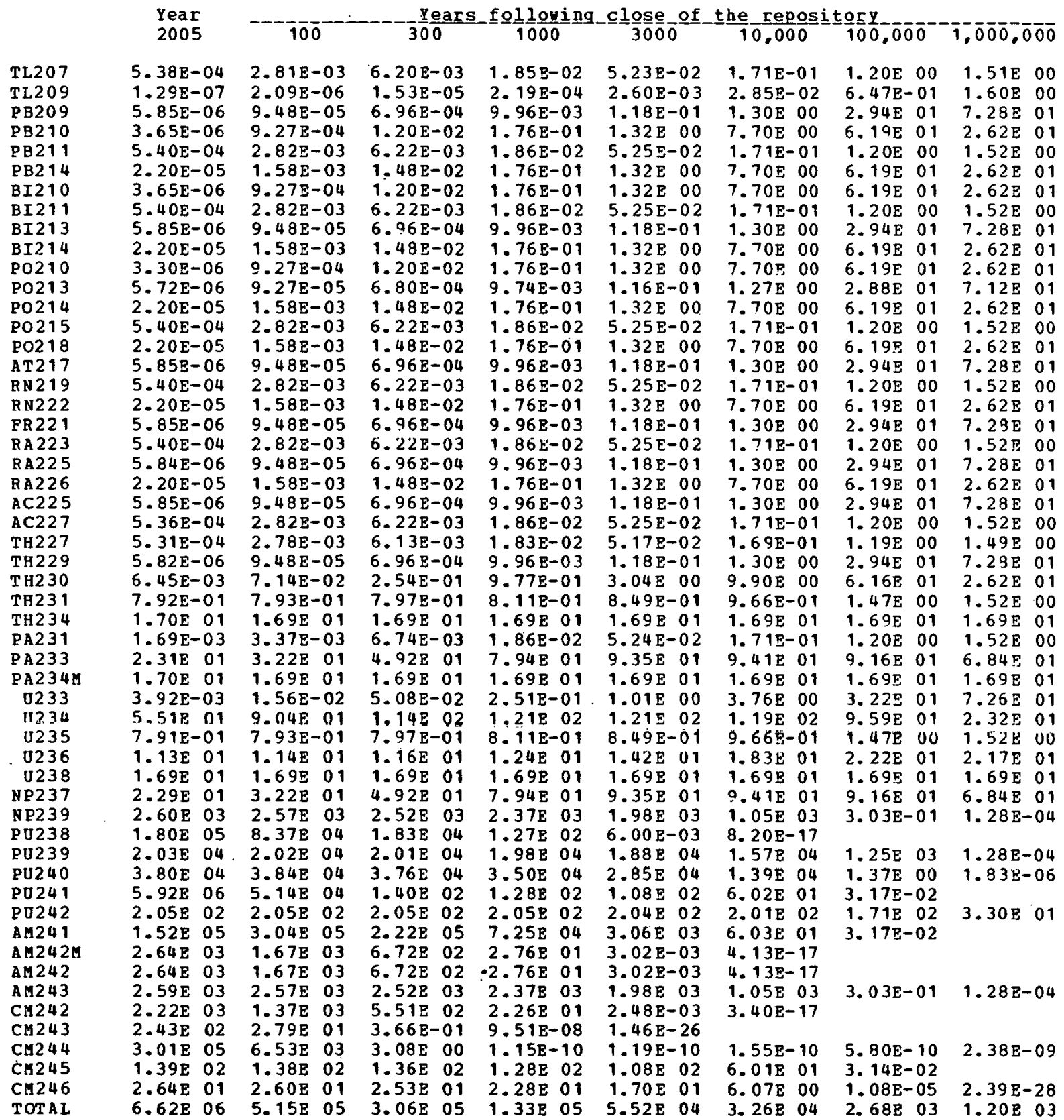


Table 4.28. Natts of accumulated heavy isotopes in the cladding wastes at a federal repository in the year 2005 as a function of age

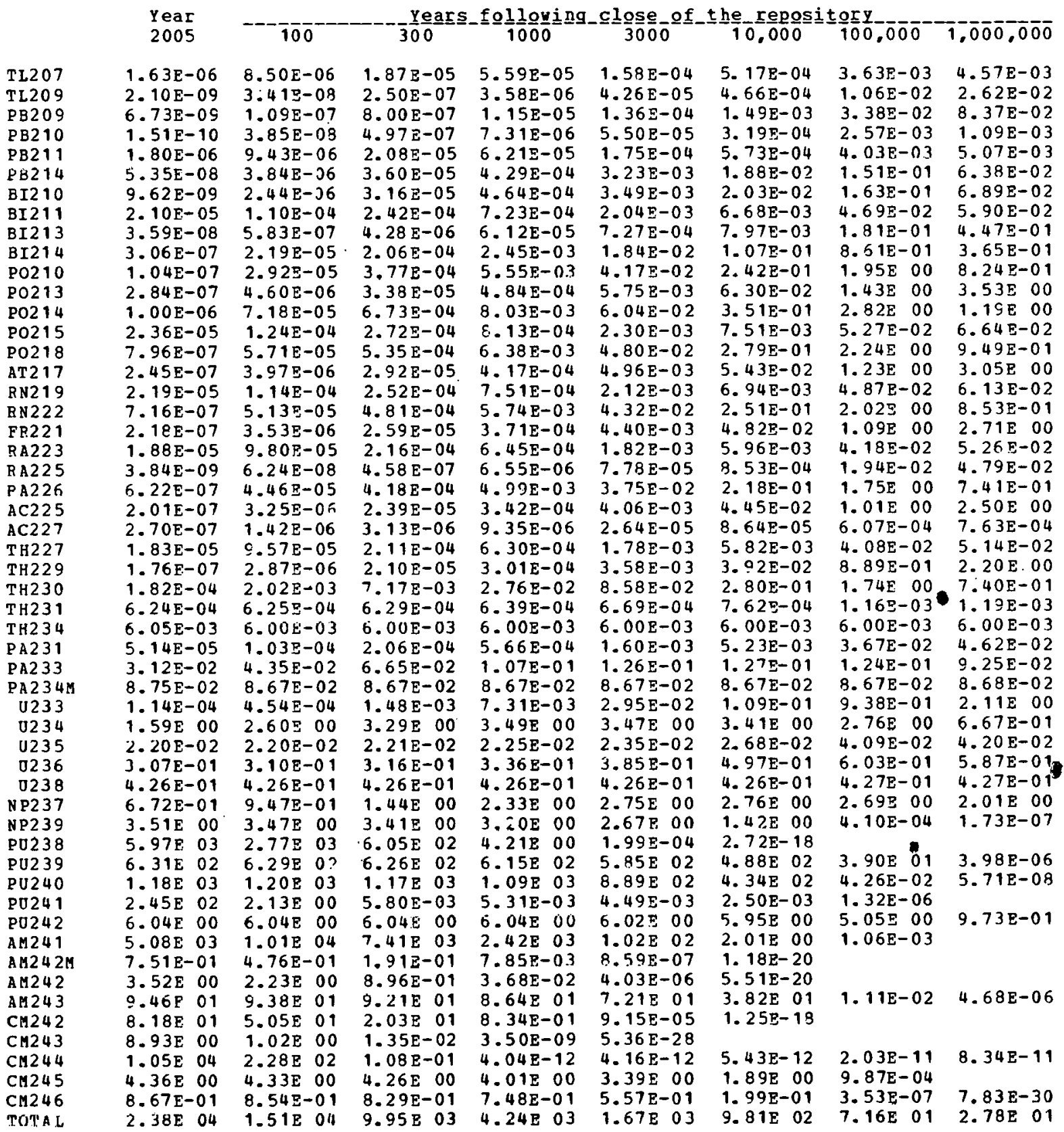


Table 4.29. Ingestion toxicity $\left(\mathrm{m}^{3}\right.$ water) of accumulated heavy isotopes in the cladding wastes at a federal repository in the year 2005 as a function of age

Year 2005 -

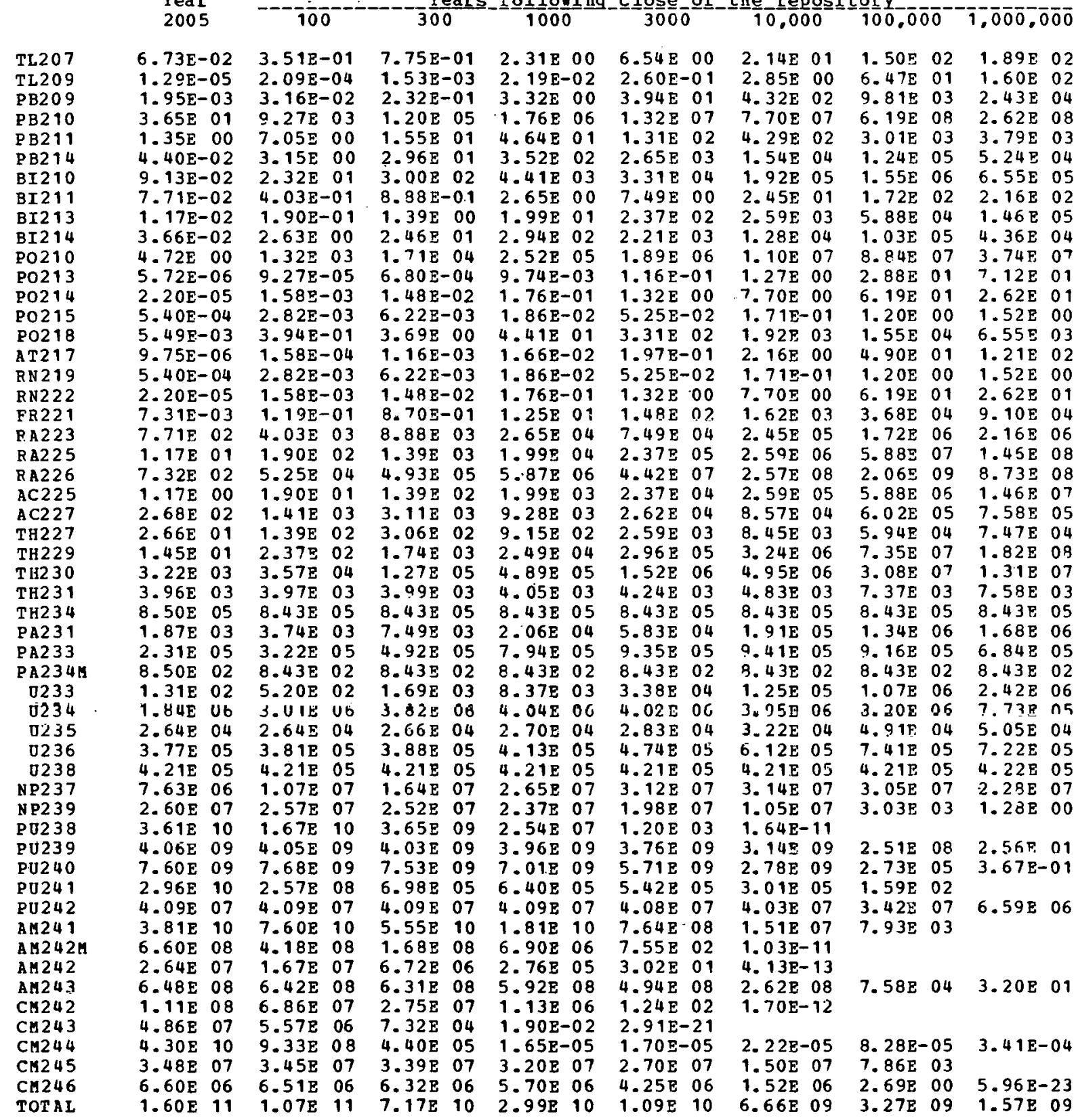


Table 4.30. Grams of accumulated fission product elements in the rladding wastes at a federal repository in the year 2005 as a function of age

\begin{tabular}{|c|c|c|c|c|c|c|c|c|c|c|c|c|c|c|c|c|}
\hline & 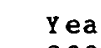 & & & & & & $=0$ & & los & & & & & & & \\
\hline & 20 & & 100 & & 30 & & 100 & & 300 & & 10 , & & 100 , & & $1,000.0$ & 00 \\
\hline GE & $.12 \mathrm{E}$ & 01 & $12 \mathrm{E}$ & 01 & $2.12 \mathrm{~g}$ & 01 & $2.12 \mathrm{E}$ & 01 & 2. $12 \mathrm{E}$ & 01 & 2. $12 \mathrm{~B}$ & 01 & $2.12 \mathrm{E}$ & 01 & $12 \mathrm{E}$ & 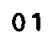 \\
\hline 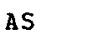 & & 00 & & & & & & 0 & & & & , & & 00 & & \\
\hline E & & 03 & $2.78 \mathrm{E}$ & 03 & & 03 & & 03 & & 03 & $2.75 \mathrm{E}$ & 03 & & 03 & & 03 \\
\hline R & $1 \mathrm{E}$ & 03 & & 03 & & 03 & & 03 & 1. $12 \mathrm{E}$ & 03 & 1. $14 \mathrm{E}$ & 03 & & 03 & & 03 \\
\hline R B & $1 E$ & 04 & 1. & 04 & $1.61 \mathrm{E}$ & 04 & IE & 04 & $E$ & 04 & $1.61 \mathrm{E}$ & 04 & & 04 & & 04 \\
\hline $5 \mathrm{R}$ & $\mathrm{E}$ & $\mathrm{C4}$ & 1. & 04 & & 04 & & 0 & & 0 & & Ou & & 04 & & 04 \\
\hline $\mathbf{Y}$ & & 04 & & 04 & & 04 & & 04 & & 04 & & 04 & & 04 & & 04 \\
\hline$R$ & & 05 & 1. & 05 & $2.00 \mathrm{D}$ & 05 & $2.00 \mathrm{E}$ & 05 & $2.00 \mathrm{E}$ & 05 & 2. & 05 & & 05 & & 05 \\
\hline B & & -01 & 1. & 00 & & 00 & & 01 & & 01 & & 0 & & 03 & & 04 \\
\hline yo & & 05 & & 0 & & 05 & & 0 & & 0 & & 0 & & 05 & & 05 \\
\hline $\mathrm{C}$ & & 04 & 4. & 04 & & 04 & 4. & 0 & & & & & & 04 & & 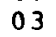 \\
\hline U & & 05 & 1. & 05 & & 05 & & 05 & & & & 05 & & 05 & & 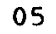 \\
\hline H & & 04 & 2. & 04 & & 04 & 2.4 & 04 & 2.4 & 0 & & 0 & & 04 & & 04 \\
\hline PD & & 04 & 8. & 0 & & 04 & 8.3 & 04 & $8.31 E$ & 0 & & 0 & & 04 & & 04 \\
\hline$c$ & & 03 & & 03 & & 03 & & (2) & & 03 & & 03 & & 03 & & 1 \\
\hline D & & 03 & & 0.3 & & 03 & & 0 & 5.1 & 0 & & 03 & & 03 & & 03 \\
\hline $\mathrm{N}$ & 7 & 0 & 7. & 01 & & 01 & & 0 & 7.6 & 0 & & & & 01 & $E$ & 01 \\
\hline & & 03 & & 03 & & 03 & & 0 & & 0 & & & & 03 & & 03 \\
\hline B & 5. & 02 & 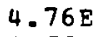 & 02 & & 02 & & 02 & & 02 & 4. & 0 & & 02 & & 02 \\
\hline$\varepsilon$ & & 06 & & 0 & & 04 & & 04 & & 0 & & 04 & & 04 & & 04 \\
\hline I & & 04 & & 04 & & 04 & & 04 & & 0 & & & & 04 & & 04 \\
\hline & & -03 & 4 & .02 & & 01 & 4. & 01 & $12 ?$ & 0 & & 0 & & 01 & & 02 \\
\hline & & 05 & & 04 & & 04 & & 04 & & 0 & & 04 & & 04 & & 0 \\
\hline & & 04 & & 05 & & 05 & & 0 & & 0 & & 05 & & 05 & & 0 \\
\hline & & 04 & & 0 & & 04 & & 04 & & 0 & & 04 & & 04 & & 04 \\
\hline$C E$ & & 05 & & 05 & & 05 & & 05 & & 0 & & 05 & & 05 & & 05 \\
\hline & & & & مu & & 04 & & & & 0 & & 0 & & 04 & & 7 \\
\hline ND & & 05 & 2 & 05 & & 05 & $\bar{E}$ & 05 & $3 E$ & 05 & & 05 & & 05 & & 05 \\
\hline PM & & 02 & & 0 & & & & & & & & & & & & \\
\hline : & $48 \mathrm{P}$ & 04 & & 04 & & 0 & & 0 & & 0 & & 0 & & 04 & & \\
\hline 0 & & 03 & & 0 & & 03 & & 0 & & 0 & & 0 & & 03 & & 03 \\
\hline & & 03 & & 03 & & 03 & & 03 & & 0 & & 0 & & 0 & & 03 \\
\hline & & 02 & & $0 ?$ & & 02 & & $0 ?$ & & 02 & & 0 & & 02 & & 02 \\
\hline DI & $2 \mathrm{E}$ & 01 & & 01 & & 01 & & 01 & $72 \mathrm{E}$ & 01 & & 01 & & 01 & & 01 \\
\hline 0 & & 00 & & 0 & & 0 & & 0 & & 0 & & 00 & & 00 & & 00 \\
\hline & & & & 0 & & 0 & & - & & 0 & & 00 & & 00 & & 00 \\
\hline OT & 8 & 06 & $E$ & 06 & $\mathrm{E}$ & 06 & $1.47 \mathrm{E}$ & 06 & $1.47 \mathrm{E}$ & 06 & $1.47 \mathrm{E}$ & 06 & $47 \mathrm{E}$ & 06 & 478 & 06 \\
\hline
\end{tabular}


Table 4.31. Curies of accumulated fission product isotopes in the cladding wastes at a federal repository in the year 2005 as a function of age

Year 2005

SE 79

SR 90

$\mathrm{Y} 90$

ZR 93.

NB $93 \mathrm{M}$

TC 99

RU106

RH106

PD 107

$S B 125$

TE1 25M

SN 126

SB126M

SB126

I 129

CS 134

CS135

CS137

BA137M

CE 144

PR144

PM 147

SM 151

EO 152

EU154

EU155

H0166M

TOTAL

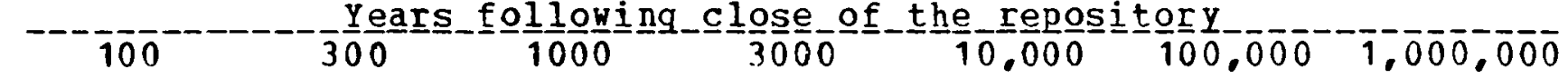

$\begin{array}{rlllllllllllll}1.94 \mathrm{E} & 01 & 1.94 \mathrm{E} & 01 & 1.93 \mathrm{E} & 01 & 1.88 \mathrm{E} & 01 & 1.75 \mathrm{E} & 01 & 6.70 \mathrm{E} & 00 & 4.55 \mathrm{E}-04 \\ 2.31 \mathrm{E} & 05 & 1.67 \mathrm{E} & 03 & 5.28 \mathrm{E}-05 & 1.98 \mathrm{E}-26 & & & & & & \\ 2.31 \mathrm{E} & 05 & 1.67 \mathrm{E} & 03 & 5.28 \mathrm{E}-05 & 1.98 \mathrm{E}-26 & & & & & \\ 8.97 \mathrm{E} & 0.1 & 8.97 \mathrm{E} & 01 & 8.97 \mathrm{E} & 01 & 8.96 \mathrm{E} & 01 & 8.93 \mathrm{E} & 01 & 8.57 \mathrm{E} & 01 & 5.65 \mathrm{E} & 01 \\ 8.94 \mathrm{E} & 01 & 8.97 \mathrm{E} & 01 & 8.97 \mathrm{E} & 01 & 8.96 \mathrm{E} & 01 & 8.93 \mathrm{E} & 01 & 8.57 \mathrm{E} & 01 & 5.65 \mathrm{E} & 01 \\ 7.27 \mathrm{E} & 02 & 7.27 \mathrm{E} & 02 & 7.25 \mathrm{E} & 02 & 7.20 \mathrm{E} & 02 & 7.04 \mathrm{E} & 02 & 5.24 \mathrm{E} & 02 & 2.77 \mathrm{E} & 01\end{array}$

$6.19 \mathrm{E} \quad 00$

$\begin{array}{lllllllllllllll}6.19 \mathrm{E} & 00 & 6.19 \mathrm{E} & 00 & 6.19 \mathrm{E} & 00 & 6.18 \mathrm{E} & 00 & 6.13 \mathrm{E} & 00 & 5.61 \mathrm{E} & 00\end{array}$

3. $23 \mathrm{E}-07$

1. $34 \mathrm{E}-07$

3. $24 \mathrm{E} 01$

3. $24 \mathrm{E} 01$

$3.21 \mathrm{E} 01$

$1.69 \mathrm{E} 00$

1. 09 E-09

1. $75 \mathrm{E} 01$

4.20E 05

$3.24 \mathrm{E} 01$

$\begin{array}{lllll}0.18 \mathrm{E} & 01\end{array}$

$3.22 \mathrm{E} 01$ 3.18E 01

$\begin{array}{lllllll}3.21 E & 01 & 3.19 E & 01 & 3.15 E & 01\end{array}$

$1.69 \mathrm{E} 00 \quad 1.69 \mathrm{E} 00 \quad 1.69 \mathrm{E} 00$

$.18 \mathrm{E} 00$

1.

3. 03E $01 \quad 1.62 \mathrm{E} 01 \quad 3.17 \mathrm{E}-02$

$3.03 \mathrm{~F} 01 \quad 1.62 \mathrm{E} \quad 01 \quad 3.17 \mathrm{E}-02$

$3.00 \mathrm{E} 01 \quad 1.61 \mathrm{E} 01 \quad 3.14 \mathrm{E}-02$

$\begin{array}{lllllll}1.75 \mathrm{E} & 01 & 1.75 \mathrm{E} & 01 & 1.75 \mathrm{E} & 01\end{array}$

$1.69 \mathrm{E} 00 \quad 1.68 \mathrm{E} 00 \quad 1.62 \mathrm{E} 00$

$5.27 \mathrm{E} 05$

$1.75 \mathrm{E} 01$

4.23E 06

$3.96 \mathrm{E} 06$

$4.13 \mathrm{E} \quad 03 \quad 3.91 \mathrm{E}-04 \quad 3.33 \mathrm{E}-24$

$\begin{array}{llllll}1.74 \mathrm{E} & 01 & 1.71 \mathrm{E} & 01 & 1.39 \mathrm{E} & 01\end{array}$

4. $19 \mathrm{E} 04$

4. $19 E 04$

4. $10 \mathrm{E} 05$

$5.90 \mathrm{E} 04$

4. $02 \mathrm{E} 02$

$2.13 \mathrm{E} 05$

$1.34 \mathrm{E} 04$

5. $17 \mathrm{E}-02$

3. $93 \mathrm{E} 05$

$3.87 \mathrm{E} 03 \quad 3.66 \mathrm{E}-04 \quad 3.12 \mathrm{E}-24$

$1.33 \mathrm{E}-06$

$2.66 \mathrm{E} \quad 04$

1. $25 \mathrm{E} 00$

\section{4}

3. $12 \mathrm{E}-13$

4. $88 \mathrm{E}-02$

1. $52 \mathrm{E} \quad 07$

$1.31 \mathrm{E} 06$
4.35E-02 2.90E-02 9.14E-03

$1.78 \mathrm{E} \quad 04 \quad 1.07 \mathrm{E} 03 \quad 1.04 \mathrm{E} \quad 03$
1. $60 E-04 \quad 4.24 E-27$

$\begin{array}{llllll}1.02 E & 03 & 7.76 E & 02 & 1.62 E & 02\end{array}$ 
Table 4.32. Watts of accumulated fission product isotopes in the cladding wastes at a federal repository

in the year 2005 as a function of age

Year 2005

SE 79

SR 90

$Y \cong 0$

$\mathrm{ZR} \quad 93$

NB $93 \mathrm{M}$

TC 99

RU106

RH 106

PD107

SB125

TF.1 25M

SN126

SR126M

SB126

I1 29

CS134

CS135

CS 137

BA 137M

CE14 4

PR 144

PM 147

SM 151

EU152

EU154

EU155

H0166M

TOTAL

\begin{tabular}{|c|c|c|c|c|c|c|}
\hline $1 \overline{0} 0$ & $30 \div 0$ & 1000 & $=\frac{3}{30}=0$ & 10,000 & $=100.000$ & $\overline{1} . \overline{0} \overline{0} \overline{0}, \overline{0} \overline{0} \overline{0}$ \\
\hline $\begin{array}{l}7.38 \mathrm{E}-03 \\
3.03 \mathrm{E} 02 \\
1.36 \mathrm{E} 03 \\
1.06 \mathrm{E}-02 \\
1.59 \mathrm{E}-02 \\
4.91 \mathrm{E}-01\end{array}$ & $\begin{array}{l}7.36 \mathrm{E}-03 \\
2.18 \mathrm{E} 00 \\
9.80 \mathrm{E} \quad 00 \\
1.06 \mathrm{E}-02 \\
1.60 \mathrm{E}-02 \\
4.91 \mathrm{E}-01\end{array}$ & $\begin{array}{l}7.30 \mathrm{E}-03 \\
6.91 \mathrm{E}-08 \\
3.11 \mathrm{E}-07 \\
1.06 \mathrm{E}-02 \\
1.59 \mathrm{E}-02 \\
4.90 \mathrm{E}-01\end{array}$ & $\begin{array}{l}7.15 \mathrm{E}-03 \\
2.59 \mathrm{E}-29 \\
1.16 \mathrm{E}-28 \\
1.06 \mathrm{E}-02 \\
1.59 \mathrm{E}-02 \\
4.87 \mathrm{E}-01\end{array}$ & $\begin{array}{l}\text { 1. } 06 E-02 \\
\text { 1. } 59 E-02 \\
\text { 4. } 76 E-01\end{array}$ & $\begin{array}{l}\text { 1. } 02 E-02 \\
\text { 1. } 52 E-02 \\
3.54 E-01\end{array}$ & $\begin{array}{l}6.70 E-03 \\
1.01 E-02 \\
1.87 E-02\end{array}$ \\
\hline $\begin{array}{l}5.14 E-04 \\
1.31 E-09 \\
2.30 E-10 \\
3.50 E-02 \\
2.19 E-01 \\
4.18 E-01 \\
1.11 E-03 \\
1.15 E-11 \\
8.49 E-03 \\
6.87 E 02 \\
1.54 E 03\end{array}$ & $\begin{array}{l}3.49 E-02 \\
2.19 E-01 \\
4.17 E-01 \\
1.11 E-03 \\
8.49 E-03 \\
6.76 F 00 \\
1.52 E 01\end{array}$ & $\begin{array}{l}3.48 E-02 \\
2.18 E-01 \\
4.15 E-01 \\
1.11 E-03 \\
8.45 E-03 \\
6.40 E-07 \\
1.44 E-06\end{array}$ & $\begin{array}{l}3.43 E-02 \\
2.15 E-01 \\
4.09 E-01 \\
1.11 E-03 \\
8.49 E-03 \\
5.46 E-27 \\
1.23 E-26\end{array}$ & $\begin{array}{l}\text { 3. } 27 \mathrm{E}-02 \\
2.05 \mathrm{E}-01 \\
3.90 \mathrm{E}-01 \\
\text { 1. } 11 \mathrm{E}-03 \\
\text { 8. } 47 \mathrm{~F}-03\end{array}$ & $\begin{array}{l}\text { 1. } 75 E-02 \\
\text { 1. } 10 F-01 \\
\text { 2. } 09 E-01 \\
\text { 1. } 11 E-03 \\
\text { 3. } 30 E-03\end{array}$ & $\begin{array}{l}3.42 E-05 \\
2.14 E-04 \\
4.08 E-04 \\
1.07 E-03 \\
6.74 E-03\end{array}$ \\
\hline $\begin{array}{l}6.85 \mathrm{E}-10 \\
4.63 \mathrm{E} 01 \\
2.24 \mathrm{E}-02 \\
2.30 \mathrm{E} 01 \\
2.63 \mathrm{E}-16 \\
5.25 \mathrm{E}-04 \\
3.96 \mathrm{E} 03\end{array}$ & $\begin{array}{l}9.41 E \quad 00 \\
2.15 E-07 \\
3.96 E-03 \\
4.68 E-04 \\
4.46 F \quad 01\end{array}$ & $\begin{array}{l}3.56 \mathrm{E}-02 \\
5.92 \mathrm{E}-25 \\
2.68 \mathrm{E}-16 \\
3.12 \mathrm{E}-04 \\
1.24 \mathrm{E} 00\end{array}$ & $\begin{array}{l}9.83 E-05 \\
1.19 E-00\end{array}$ & $\begin{array}{l}1.72 E-06 \\
1.15500\end{array}$ & $\begin{array}{l}\text { 4. } 56 F-29 \\
7.28 F-01\end{array}$ & $4.44 E-0 ?$ \\
\hline
\end{tabular}


Table 4.33: Ingestion toxizity ( $\mathrm{m}^{3}$ water) of accumulated fission product isotopes in the cladding wastes at a federal repository

in the year 2005 as a function of age

Year 2005

SE 79

SR 90

Y 90

ZR 93

NB $93 M$

TC 99

RU106

RH106

PD 107

SB 125

TE125M

SN 126

SB126M

SB126

I 129

$\operatorname{cs} 134$

CS135

$\operatorname{Cs} 137$

BA137M

CE144

PR 144

PM 147

SM 151

EU152

E.U154

EU155

HO166M

TOTAL

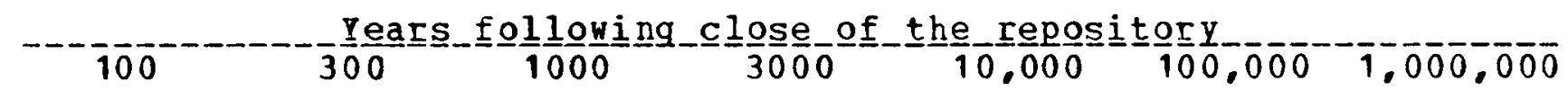

$1.95 \mathrm{E} 01$

1.94E 01

$\begin{array}{llll}1.94 \mathrm{E} & 01 & 1.93 \mathrm{E} & 01\end{array}$

$5.55 \mathrm{E} \quad 0 \subseteq \quad 1.76 \mathrm{E} \quad 02$

9.08E 12

1. $36 \mathrm{E} 11$

1. $12 \mathrm{E} \quad 05$

$7.70 \mathrm{E} \quad 11$

1. $16 \mathrm{E} 10$

$1.12 \mathrm{E} 05$

1. $15 \mathrm{E} 05$

$3.64 \mathrm{E} 06$

$2.24 \pm 05$

$3.64 \mathrm{E} \quad 06$

8.33E 0T 2.64E 00

$1.12 \mathrm{E} 0 \mathrm{E} \quad 1.12 \mathrm{E} 05$

$2.24 \mathrm{E} 05 \quad 2.24 \mathrm{E} 05$

$3.63 \mathrm{E} 06 \quad 3.62 \mathrm{E} 06$

$1.88 \mathrm{E} 01$

$6.59 \mathrm{E}-20$

$9.45 \mathrm{E} 09$.

$9.45 \mathrm{E} \quad 04$

$6.19 E \quad 03$

$4.56 \mathrm{E} \quad 08$

$6.19 E \quad 03$

$3.23 E-03$

1. $34 \mathrm{E}-03$

$3.24 \mathrm{E} 01$

$3.24 \mathrm{E} \quad 01$

$3.24 \mathrm{E} 01$

3. $23 E 01$

$2.81 \mathrm{E} \quad 07$

$5.86 \mathrm{E} \quad 10$

1. $75 \mathrm{E} 05$

$2.12 \mathrm{E} 11$

3. $96 \mathrm{E} 06$

4. 19E 09

4. 19504

$2.05 \mathrm{E} 09$

$1.47 \mathrm{E} 08$

$5.03 \mathrm{E} 06$

$1.06 \mathrm{E} \quad 10$

$6.68 \mathrm{E} \quad 07$

$3.24 \mathrm{E} 01$

3. $21 \mathrm{E} 01$

2.81E 07

1. $21 E-04$

1. $75 \mathrm{E} 05$

$2.10 \mathrm{E} \quad 10$

3. $93 \mathrm{E} 05$

$6.19 \mathrm{E} \quad 03 \quad 6.19 \mathrm{E} \quad 03$

$9.89 \mathrm{E}-22$

1. 12E 05

$2.24 \mathrm{E} 05$

$3.60 \mathrm{E} 06$

1. 12F 05

2. $23 \mathrm{E} 05$

3. $52 \mathrm{E} 06$

$6.70 \mathrm{~F}: 00$

4. $55 E-04$

1. 75F 01

$6.70 \mathrm{~g}-00$

. 07E 05

2. 14E 05

$7.06 \mathrm{~F} 04$

2. $62 \mathrm{E} 06$

$1.41 E 05$

$1.38 \mathrm{E} 05$

$6.64 \mathrm{E}-03$

$6.65 \mathrm{E} 07$

$1.56 \mathrm{E} \quad 04$

$1.40 \mathrm{E} \quad 08$

$1.56 \mathrm{E}-09$

5. $17 \mathrm{E}-02$

$9.51 \mathrm{E} 12$

$8.03 E 11$

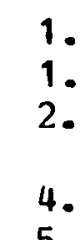$$
\begin{aligned}
& 1 . \\
& 1 . \\
& 2 \\
& 4
\end{aligned}
$$

$1.74 \mathrm{E} 05$

$1.71 E 05$

1. $39 \mathrm{~F} 05$

4. 35E-02 2.90E-02 9.14E-03.

$\begin{array}{llll}5.89 \mathrm{E} & 03 & 3.23 \mathrm{E} & 07\end{array}$

$3.22 \mathrm{E} 07$

1. $60 \mathrm{E}-04$

4. $245-27$

$3.21 \mathrm{E} \quad 07 \quad 3.11 \mathrm{E} \quad 07$

$2.75 \mathrm{E} \quad 07$ 
the upper range of alpha-emitting isotopes in naturally occurring deposits. It is reasonable that wastes containing less alpha activity than $10 \mathrm{nCi} / \mathrm{g}$ be regarded as disposable in carefully selected burial grounds, whereas alpha wastes must be stored in special repositories that offer maximal assurance of permanent containment. These wastes arise at fuel reprocessing plants and consist of an assortment of materials similar to the low-level TRU wastes discussed below. About $10,000 \mathrm{ft}^{3}$ of intermediate-level TRU wastes is generated per ton of plutonium or ${ }^{233} \mathrm{U}$ processed. These wastes contain an average of $0.025 \mathrm{~g}$ of plutonium or uranium per cubic foot, ${ }^{26}$ plus an assumed $0.025 \%$ of the fission products in the spent fuel.

Shipments and accumulations of intermediate-level TRU solid wastes at one or more federal repositories are presented in Table 4.34. The wastes are compacted on the same schedule as is assumed for the lowlevel TRU wastes below. They are packaged in steel canisters $2 \mathrm{ft}$ in diam by $10 \mathrm{ft}$ long and transported by motor freight five years following the date of waste generation. Each shipment consists of three such canisters (containing a total of $75 \mathrm{ft}^{3}$ of waste) in a lightly shielded cask.

The accumulated mass, radioactivity, thermal power, and ingestion toxicity of the intermediate-level TRU wastes as a function of time following the year 2005 are presented in Tables 4.35 through 4.38 for actinides, and in Tables 4.39 through 4.42 for fission products.

\subsection{Low-Level Transuranic Wastes}

Low-level TRU wastes are defined as those solid materials that contain plutonium or other long-lived alpha emitters in concentrations greater than $10 \mathrm{nCi} / \mathrm{g}$ and yet have sufficiently low external radiation levels that they can be handled directly without supplementary shielding (surface dose rates less than $10 \mathrm{mrem} / \mathrm{hr}$ ).

Low-level TRU wastes arise principally at fuel preparation and fabrication plants, and to a lesser extent at fuel reprocessing plants. ${ }^{26,27}$ They consist of a wide assortment of solid materials, including iteme 
Table 4.34. Projected annual accumulation of intermediate-level transuranium waste at a federal repository

\begin{tabular}{|c|c|c|c|c|c|}
\hline Year & $\begin{array}{l}\text { * * * } \\
\text { Volume } \\
\text { (Thousands } \\
\text { of } \mathrm{m}^{3} \text { ) }\end{array}$ & $\begin{array}{l}\text { Annual } \\
\text { Actinide } \\
\text { Mass } \\
\text { (kq) }\end{array}$ & $\begin{array}{l}\text { dition } \\
\text { Radio- } \\
\text { activity } \\
\text { (MCi) }\end{array}$ & $\begin{array}{l}\text { * * * } \\
\text { Thermal } \\
\text { Power } \\
\text { (kW) }\end{array}$ & $\begin{array}{c}\text { Arnua } 1 \\
\text { Number } \\
\text { of } \\
\text { Sh Epments }\end{array}$ \\
\hline $\begin{array}{l}1984 \\
1985 \\
1986 \\
1987 \\
1988 \\
1989 \\
1990 \\
1991 \\
1992 \\
1993 \\
1994 \\
1995 \\
1996 \\
1997 \\
1998 \\
1999 \\
2000 \\
2001 \\
2002 \\
2003 \\
2004 \\
2005\end{array}$ & $\begin{array}{l}0.0 \\
0.0 \\
0.3 \\
0.7 \\
0.8 \\
0.7 \\
1.0 \\
1.0 \\
1.1 \\
1.0 \\
1.3 \\
1.4 \\
1.7 \\
1.8 \\
2.1 \\
2.4 \\
2.9 \\
3.1 \\
3.5 \\
3.7 \\
3.7 \\
3.9\end{array}$ & $\begin{array}{c}0.0 \\
0.0 \\
0.8 \\
2.6 \\
2.8 \\
3.0 \\
4.3 \\
6.7 \\
7.0 \\
8.9 \\
11.0 \\
12.1 \\
14.8 \\
15.8 \\
18.7 \\
21.4 \\
25.3 \\
27.2 \\
30.6 \\
32.9 \\
32.6 \\
34.2\end{array}$ & $\begin{array}{l}0.0 \\
0.0 \\
0.0 \\
0.1 \\
0.2 \\
0.2 \\
0.2 \\
0.4 \\
0.3 \\
0.4 \\
0.5 \\
0.6 \\
0.7 \\
0.8 \\
0.9 \\
1.0 \\
1.1 \\
1.1 \\
1.3 \\
1.5 \\
1.3 \\
1.3\end{array}$ & $\begin{array}{l}0.0 \\
0.0 \\
0.2 \\
0.5 \\
0.5 \\
0.6 \\
0.8 \\
1.2 \\
1.2 \\
1.3 \\
1.7 \\
1.9 \\
2.3 \\
2.5 \\
3.0 \\
3.2 \\
3.7 \\
3.9 \\
4.4 \\
4.9 \\
4.5 \\
4.2\end{array}$ & $\begin{array}{r}0 \\
2 \\
150 \\
340 \\
372 \\
324 \\
+54 \\
777 \\
501 \\
475 \\
589 \\
645 \\
790 \\
840 \\
995 \\
1143 \\
1349 \\
1449 \\
1632 \\
1755 \\
1740 \\
1825\end{array}$ \\
\hline
\end{tabular}

\begin{tabular}{|c|c|c|c|c|c|c|c|}
\hline $\begin{array}{l}\text { Volume } \\
\text { (Thousands } \\
\text { of } \mathrm{m}^{3} \text { ) }\end{array}$ & $\begin{array}{l}\text { Actinide } \\
\text { Mass } \\
(\mathrm{kg})\end{array}$ & $\begin{array}{l}\text { Radio- } \\
\text { activity } \\
\text { (MCi) }\end{array}$ & $\begin{array}{l}\text { Thermal } \\
\text { Power } \\
\text { (kW) }\end{array}$ & $\begin{array}{l}\mathrm{Haz} \\
\mathrm{Met} \\
\mathrm{Air}\end{array}$ & $\begin{array}{l}\text { ard, } \\
\text { ers }\end{array}$ & $\begin{array}{l}\text { Cubic } \\
\text { at RCG } \\
\text { Hater }\end{array}$ & \\
\hline $\begin{array}{l}0.0 \\
0.0 \\
0.3 \\
1.0 \\
1.8 \\
2.5 \\
3.5 \\
4.5 \\
5.6 \\
6.6 \\
7.8 \\
9.2 \\
10.9 \\
12.7 \\
14.8 \\
17.2 \\
20.1 \\
23.1 \\
26.6 \\
30.3 \\
34.0 \\
37.9\end{array}$ & $\begin{array}{r}0 \\
0 \\
1 \\
3 \\
6 \\
9 \\
13 \\
20 \\
27 \\
36 \\
47 \\
59 \\
74 \\
90 \\
108 \\
130 \\
155 \\
182 \\
213 \\
246 \\
279 \\
313\end{array}$ & $\begin{array}{r}0.00 \\
0.00 \\
0.05 \\
0.18 \\
0.32 \\
0.46 \\
0.67 \\
0.97 \\
1.25 \\
1.58 \\
1.99 \\
2.44 \\
2.99 \\
3.58 \\
4.27 \\
4.99 \\
5.81 \\
6.66 \\
7.64 \\
8.71 \\
9.62 \\
10.40\end{array}$ & $\begin{array}{l}0.0 \\
0.0 \\
0.2 \\
0.6 \\
1.0 \\
1.5 \\
2.1 \\
3.1 \\
4.0 \\
5.0 \\
6.3 \\
7.7 \\
9.4 \\
11.3 \\
13.4 \\
15.6 \\
19.2 \\
20.8 \\
23.9 \\
27.2 \\
30.0 \\
32.4\end{array}$ & $\begin{array}{l}1.2 \mathrm{E} \\
3.3 \mathrm{E} \\
5.9 \mathrm{E} \\
2.4 \mathrm{E} \\
4.3 \mathrm{E} \\
6.4 \mathrm{E} \\
9.4 \mathrm{E} \\
1.4 \mathrm{E} \\
1.8 \mathrm{E} \\
2.3 \mathrm{E} \\
2.9 \mathrm{E} \\
3.6 \mathrm{E} \\
4.5 \mathrm{E} \\
5.4 \mathrm{E} \\
6.5 \mathrm{E} \\
7.6 \mathrm{E} \\
8.9 \mathrm{E} \\
1.0 \mathrm{E} \\
1.2 \mathrm{E} \\
1.4 \mathrm{E} \\
1.5 \mathrm{E} \\
1.7 \mathrm{E}\end{array}$ & $\begin{array}{l}13 \\
13 \\
15 \\
16 \\
16 \\
16 \\
16 \\
17 \\
17 \\
17 \\
17 \\
17 \\
17 \\
17 \\
17 \\
17 \\
17 \\
18 \\
18 \\
18 \\
18 \\
18\end{array}$ & $\begin{array}{l}4.1 \mathrm{E} \\
1.2 \mathrm{E} \\
2.1 \mathrm{E} \\
8.4 \mathrm{E} \\
1.5 \mathrm{E} \\
2.2 \mathrm{E} \\
3.3 \mathrm{E} \\
4.8 \mathrm{E} \\
6.2 \mathrm{E} \\
7.9 \mathrm{E} \\
1.0 \mathrm{E} \\
1.2 \mathrm{E} \\
1.5 \mathrm{E} \\
1.8 \mathrm{E} \\
2.2 \mathrm{E} \\
2.6 \mathrm{E} \\
3.0 \mathrm{E} \\
3.4 \mathrm{E} \\
4.0 \mathrm{E} \\
4.5 \mathrm{E} \\
5.0 \mathrm{E} \\
5.5 \mathrm{E}\end{array}$ & $\begin{array}{l}07 \\
08 \\
10 \\
10 \\
11 \\
11 \\
11 \\
11 \\
11 \\
11 \\
12 \\
12 \\
12 \\
12 \\
12 \\
12 \\
12 \\
12 \\
12 \\
12 \\
12 \\
12\end{array}$ \\
\hline $\begin{array}{l}37.9 \\
37.9 \\
37.9 \\
37.9 \\
37.9 \\
37.9 \\
37.9 \\
37.9 \\
37.9\end{array}$ & $\begin{array}{l}313 \\
313 \\
313 \\
313 \\
313 \\
313 \\
313 \\
313 \\
313\end{array}$ & $\begin{array}{l}0.86 \\
0.11 \\
0.05 \\
0.02 \\
0.01 \\
0.01 \\
0.00 \\
0.00 \\
0.00\end{array}$ & $\begin{array}{l}6.5 \\
3.2 \\
1.5 \\
0.6 \\
0.4 \\
0.1 \\
0.0 \\
0.0 \\
0.0\end{array}$ & $\begin{array}{l}9.8 \mathrm{E} \\
7.5 \mathrm{E} \\
4.6 \mathrm{E} \\
3.0 \mathrm{E} \\
1.9 \mathrm{E} \\
7.5 \mathrm{E} \\
1.1 \mathrm{E} \\
2.5 \mathrm{E} \\
2.1 \mathrm{E}\end{array}$ & $\begin{array}{l}17 \\
17 \\
17 \\
17 \\
17 \\
16 \\
16 \\
15 \\
15\end{array}$ & $\begin{array}{l}4.9 \mathrm{E} \\
2.7 \mathrm{E} \\
1.0 \mathrm{E} \\
3.9 \mathrm{E} \\
2.4 \mathrm{E} \\
1.3 \mathrm{E} \\
9.5 \mathrm{E} \\
1.1 \mathrm{E} \\
6.5 \mathrm{E}\end{array}$ & $\begin{array}{l}11 \\
10 \\
10 \\
09 \\
09 \\
09 \\
08 \\
09 \\
08\end{array}$ \\
\hline
\end{tabular}


Table 4.35. Grams of accumulated heavy elements in intermediateradiation-level TRU wastes at a federal repository

i. the year 2005 as a function of age

\begin{tabular}{|c|c|c|c|c|c|c|c|c|}
\hline & ear & & Ye & E은 1 음 & cㅢㅢ으으. & h $\underline{\text { e }}$ & & \\
\hline & & 100 & 300 & 1000 & 3000 & 10.000 & 100,000 & $1.000,000$ \\
\hline$E$ & $1.17 \mathrm{E} 01$ & $-18 \mathrm{E} \quad 02$ & $2.94 \mathrm{E} \quad 02$ & $6.53 \mathrm{E} \quad 02$ & $1.07 \mathrm{E} \quad 03$ & $1.86 F \quad 03$ & $4.08 \mathrm{E} 03$ & $6.74 \mathrm{E}$ \\
\hline L & $E-10$ & $3.94 E-10$ & $7.48 E-11$ & $5.64 \mathrm{E}-11$ & $51 F-10$ & $E-10$ & $E-09$ & $E-09$ \\
\hline 3 & -03 & $+E-02$ & $3.50 \mathrm{E}-02$ & $E-02$ & $8-01$ & E 00 & $\Xi 02$ & 03 \\
\hline I & -08 & 00 & E- & 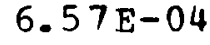 & 2 & $8-01$ & 2 & 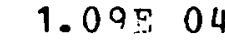 \\
\hline & -10 & $E-08$ & 9. $31 \mathrm{E}-07$ & $E-05$ & 5 & $E-04$ & $;-03$ & $E-$ \\
\hline & -18 & $3.55 E-17$ & $4 z-16$ & $E-15$ & $3.30 F-14$ & 3.4 & -12 & \\
\hline & -10 & $.62 \mathrm{E}-09$ & $1 E-08$ & $E-07$ & $1 F-06$ & $E-0.5$ & 04 & $E-05$ \\
\hline & & $E-13$ & $1 E-12$ & $E-11$ & & 3. & & -07 \\
\hline & & & $4 E-03$ & $\Xi-02$ & 1 & $E 00$ & 01 & \\
\hline & 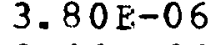 & $E-05$ & $25-05$ & $E-04$ & $E-04$ & $:-03$ & $E-03$ & $E-02$ \\
\hline H & 1 & $.78 \mathrm{~F}, 00$ & $2 \mathrm{E}$ & $\mathrm{E} 01$ & F: 01 & P 02 & E 03 & $\begin{array}{lll}E & 03\end{array}$ \\
\hline $\mathrm{A}$ & 2 & $E-02$ & $3 E-02$ & $E-01$ & $4 E-01$ & $3 \mathrm{E} \quad 00$ & $0 E \quad 01$ & 501 \\
\hline U & E 07 & $6 E \quad 07$ & $6 \mathrm{E}$ & B 07 & $7 F \quad 07$ & 7507 & 8507 & 207 \\
\hline $\mathrm{P}$ & E 04 & $2 \mathrm{E} \quad 04$ & $2 E$ & E 04 & $5.62 \mathrm{E} \quad 04$ & $3 \mathrm{E} \quad 04$ & $7 \mathrm{E} \quad 04$ & E 04 \\
\hline $\mathrm{U}$ & 05 & $2.13 \mathrm{E} \quad 05$ & 10505 & E 05 & $5 E \quad 05$ & 1 F 05 & E 04 & E 03 \\
\hline & 04 & $3.21 \mathrm{E} \quad 04$ & 2.40504 & E 03 & 3403 & $7 \mathrm{E} \quad 0 ?$ & $E-01$ & $\mathrm{E}-06$ \\
\hline & 02 & $1.71 \mathrm{E} \quad 01$ & $7.42 \mathrm{E} 00$ & $E 00$ & $5.81 \mathrm{E} 00$ & $3.13 \mathrm{~F} 00$ & $E-02$ & 2 \\
\hline & $=-08$ & $5 E-19$ & $045-19$ & $E-19$ & $5 F-20$ & $E-20$ & $\mathrm{E}-21$ & 37 \\
\hline & $F-05$ & $.83 E-06$ & $98 E-06$ & $3 \mathrm{E}-06$ & $1 \mathrm{E}-07$ & 3. $39 \mathrm{E}-10$ & $78 E-17$ & $1.98 \mathrm{E}-32$ \\
\hline & & & & & & & & \\
\hline 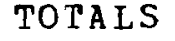 & $9 \mathrm{E} \quad 07$ & $.59 \mathrm{E} \quad 07$ & 2.59507 & $2.59 \pm 07$ & $2.5 \mathrm{C} E 07$ & 2.50507 & $2.59 \mathrm{E} 07$ & $2.59 E \quad 07$ \\
\hline
\end{tabular}


Table 4.36. Curies of accumulated heavy isotopes in intermediateradiation-level TRO wastes at a federal repository

in the year 2005 as a function of age

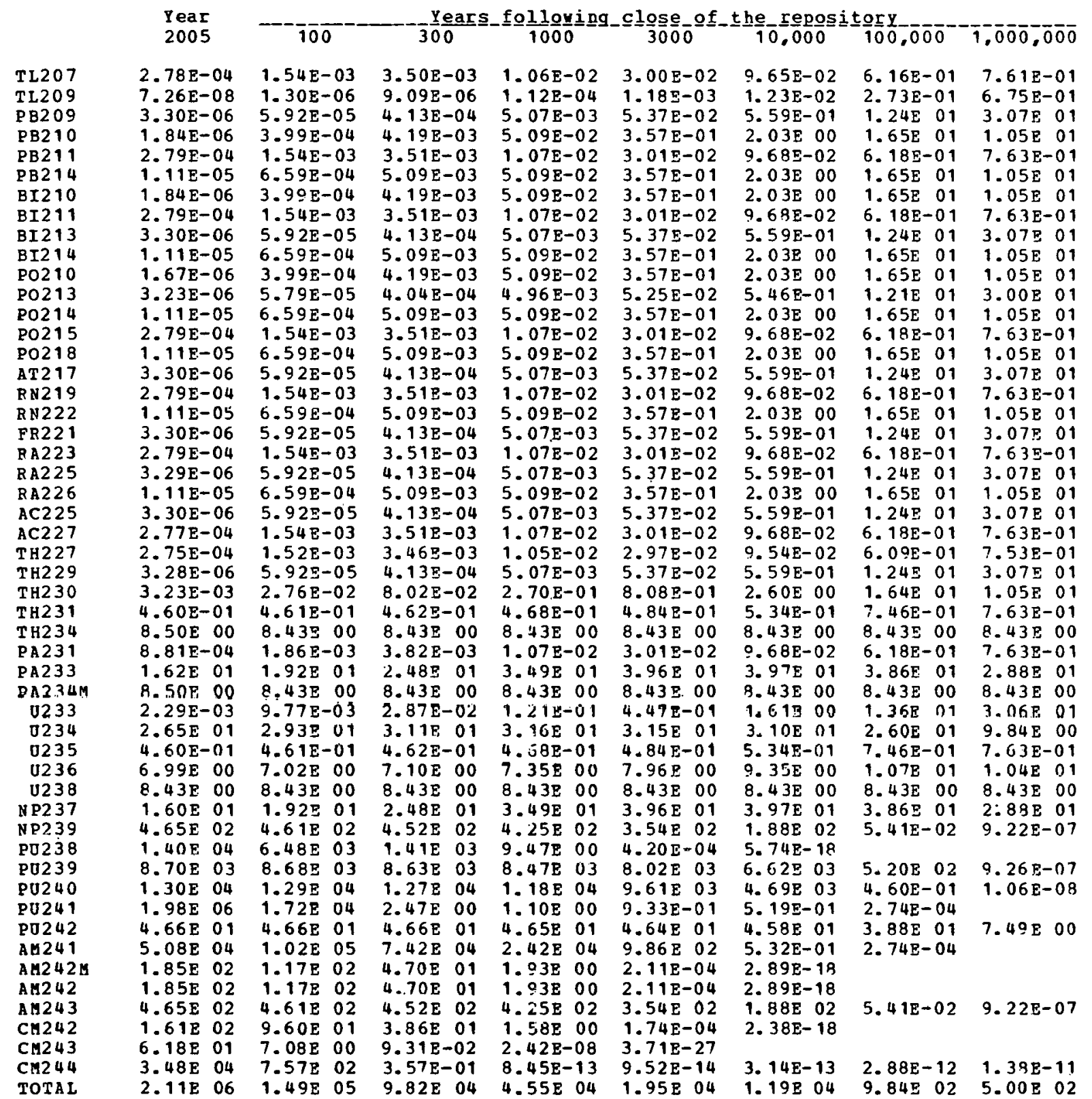


Table 4.37. Watts of accumulated heavy isotopes in intermediateradiation-level TRU wastes at a federal repository

in the year 2005 as a function of age

Year

2005

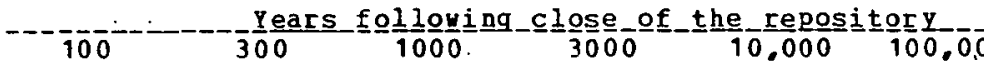
$\overline{0}-\overline{1}, \overline{0} \overline{0} \overline{0}, \overline{0} \overline{0} \overline{0}$

TL207

TL200

PB209

PB210

PB211

PB214

BI210

BI211

BI 213

BI2 14

PO210

P0213

PO 214

P0215

PO218

AT2 17

RN219

RN222

FR221

R.A223

RA2 25

RA2 26

AC225

AC2 27

TH2 27

TH229

$\mathrm{TH} 230$

TH231

TH234

PA231

PA233

PA2 $34 \mathrm{M}$

प233

U234

v2 35

U2 36

U238

NP237

NP2 39

PU238

PU23?

PU240

PU241

PU 242

A 4241

A 242 M

AM 242

AM 243

CM 242

CM 243

CM2 44

TOTAL
8. $42 \mathrm{E}-07$

$1.19 \mathrm{E}-0 \mathrm{O}$

3. $80 \mathrm{E}-09$

$7.65 \mathrm{E}-11$

$9.34 \mathrm{E}-07$

2. $70 F-08$

4. $85 \mathrm{E}-09$

$1.09 F-05$

$2.03 \mathrm{E}-08$

$1.54 \mathrm{E}-07$

$5.25 \mathrm{E}-08$

1. $60 \mathrm{E}-07$

$5.05 \mathrm{E}-07$

1. $22 \mathrm{E}-05$

4. $02 \mathrm{E}-07$

1. $38 \mathrm{E}-07$

$1.13 E-05$

3. $61 \mathrm{E}-07$

1. $23 \mathrm{E}-07$

$9.70 E-06$

2. 17E-09

$3.14 \mathrm{E}-07$

1. $13 E-07$

$1.40 \mathrm{E}-07$

9. $46 \mathrm{E}-06$

$.03 \mathrm{E}-08$

9. $13 E-05$

3. $63 E-04$

$3.02 \mathrm{E}-03$

$2.69 \mathrm{E}-05$

2. $19 \mathrm{~F}-02$

4. $37 E-02$

6. $66 \mathrm{E}-05$

$7.64 E-01$

1. 28E-02

$1.89 \mathrm{E}-01$

2. 13E-01

4. $71 \mathrm{E}-01$

$6.29 \mathrm{E}-01$

$4.63 \mathrm{E} 02$

$2.70 \mathrm{E} \quad 02$

$4.04 \mathrm{E} 02$

$8.24 \mathrm{E} 01$

$1.38 \mathrm{E} 00$

$1.69 \mathrm{E} 03$

5. $26 \mathrm{E}-02$

$2.46 E-01$

1.70E 01

5.93E 00

$2.27 \mathrm{E} 00$

$1.22 \mathrm{E} \mathrm{OJ}$

4. 16E 03
4. $65 \mathrm{E}-06$

$2.13 E-09$

$6.81 \mathrm{~s}-08$

$1.65 \mathrm{E}-1) 8$

$5.16 \mathrm{E}-1) 6$

1. $60 \mathrm{E}-06$

1. $05 E-06$

$6.01 \mathrm{E}-05$

3. $64 \mathrm{E}-07$

$9.17 \mathrm{E}-06$

1.25E-05

$2.88 \mathrm{E}-06$

3. $00 \mathrm{E}-05$

$6.76 \mathrm{E}-05$

$2.39 E-05$

$2.48 \mathrm{E}-06$

$6.25 E-05$

$2.15 E-05$

2. $20 E-06$

$5.36 \mathrm{Q}-05$

3. $90 \mathrm{E}-0^{\circ}$

$1.86 \mathrm{E}-0 \mathrm{5}$

$2.03 \mathrm{~B}-06$

$7.78 \mathrm{E}-07$

$5.24 \mathrm{E}-05$

$1.798-05$

$7.79 z-04$

$3.63 \mathrm{E}-04$

$3.005-03$

$5.68 E-05$

$2.59 \mathrm{E}-02$

4. $34 \mathrm{E}-02$

$2.84 \mathrm{E}-04$

$8.435-01$

$1.285-02$

1. $90 \mathrm{E}-01$

2. $13 \mathrm{E}-01$

$5.63 \mathrm{E}-01$

$6.23 \mathrm{E}-01$

$2.15 \mathrm{E} 02$

2.70502

$4.03 \mathrm{E} \quad 02$

7. $148-01$

1.38500

$3.39 \mathrm{E} 03$

3. $33 \mathrm{E}-02$

$1.56 \mathrm{E}-01$

$1.68 \mathrm{E} 01$

$3.54 \mathrm{E} \quad 00$

$2.60 \mathrm{E}-01$

$2.65 \mathrm{E} 01$

$4.33 \mathrm{E} \quad 03$
$1.06 \mathrm{E}-05 \quad 3.21 \mathrm{E}-05$

$1.49 \mathrm{E}-07 \quad 1.82 \mathrm{E}-06$

$4.75 \mathrm{E}-07 \quad 5.83 \mathrm{E}-06$

$1.74 \mathrm{E}-07 \quad 2.11 \mathrm{E}-06$

$1.17 \mathrm{E}-05 \quad 3.56 \mathrm{E}-05$

$1.24 \mathrm{E}-05$

1. $10 \mathrm{E}-05$

1. $37 \mathrm{E}-04$

$2.54 \mathrm{E}-06$

7. $08 \mathrm{E}-05$

1. $32 \mathrm{E}-04$

$2.01 \mathrm{E}-05$

2. $32 \mathrm{E}-04$

$1.545-04$

$1.84 \mathrm{E}-04$

$1.73 \mathrm{E}-05$

$1.42 \mathrm{E}-04$

$1.66 \mathrm{E}-04$

$1.54 \mathrm{E}-05$

$1.22 \mathrm{E}-04$

2. $72 \mathrm{E}-07$

$1.44 \mathrm{E}-04$

$1.42 \mathrm{E}-05$

$1.77 E-06$

$1.19 \mathrm{E}-04$

1. $25 \mathrm{E}-05$

2. $27 \mathrm{E}-03$

3. $65 E-04$

3. $00 \mathrm{E}-03$

$1.16 \mathrm{E}-04$

$3.36 E-02$

4. $34 \mathrm{E}-02$

8. $36 E-04$

8. $96 \mathrm{E}-01$

1. $23 \mathrm{E}-02$

$1.92 \mathrm{E}-01$

2.13 $5-01$

7. $29 \mathrm{E}-01$

$6.12 \mathrm{~b}-01$

4.67E 01

$2.68 \mathrm{E} \quad 02$

$3.95 \mathrm{E} \quad 02$

$1.02 \mathrm{E}-04$

$1.38 \mathrm{E} 00$

$2.48 \mathrm{E} 03$

$1.34 \mathrm{E}-02$

$6.27 \mathrm{E}-02$

$1.65 \mathrm{E} 01$

$1.42 \mathrm{E} 00$

1. $24 E-04$

1. $34 \mathrm{E}-04$

4. 15E-04

$3.12 \mathrm{E}-05$

$7.08 \mathrm{E}-04$

1. $60 \mathrm{E}-03$

$2.46 \mathrm{E}-04$

$2.32 \mathrm{E}-03$

$4.67 \mathrm{E}-04$

1. $84 \mathrm{E}-03$

$2.12 E-04$

4. $31 E-04$

1. $66 \mathrm{E}-03$

$1.89 \mathrm{E}-04$

3. $70 \mathrm{E}-04$

3. $34 \mathrm{~F}-06$

$1.44 \mathrm{E}-03$

$1.74 \mathrm{E}-04$

5. $37 \mathrm{E}-06$

3. $62 \mathrm{E}-04$

1. $53 \mathrm{E}-04$

$7.64 \mathrm{E}-03$

$3.69 \mathrm{E}-04$

$3.00 E-03$

3. $25 \mathrm{E}-04$

4. $72 \mathrm{E}-02$

4. $34 \mathrm{E}-02$

3. $52 \mathrm{E}-03$

9. 10E-01

1. $30 \mathrm{E}-02$

1. $99 \mathrm{E}-01$

2.13E-01

$1.03 \mathrm{E} 00$

5. $74 E-01$

3. $14 \mathrm{E}-01$

2. $63 \mathrm{E} 02$

$3.67 \mathrm{E} 02$

4. $58 \mathrm{E}-05$

1. $37 \mathrm{E} 00$

$8.08 \mathrm{E} \quad 02$

$5.50 E-04$

$2.58 \mathrm{E}-03$

1.55E 01

$3.42 \mathrm{E}-03$

1.25E-02

5. $84 \mathrm{E}-02$

8. $89 \mathrm{E}-10$

$2.96 \mathrm{E}-14$

$1.46 \mathrm{E} 03$

$9.08 \mathrm{E}-05$

1. $93 z-05$

$6.18 E-05$

$1.48 \mathrm{E}-05$

$1.01 \mathrm{E}-04$

$8.69 \mathrm{E}-04$

$9.39 \mathrm{E}-04$

1. $17 \mathrm{E}-03$

$3.30 \mathrm{E}-04$

$4.97 \mathrm{E}-03$

1. $12 E-02$

$2.61 \mathrm{E}-03$

$1.62 \mathrm{E}-02$

1. $32 \mathrm{E}-03$

$1.29 \mathrm{E}-02$

$2.25 E-03$

$1.22 \mathrm{E}-03$

$1.16 \mathrm{E}-02$

$2.00 \mathrm{E}-03$

$1.05 \mathrm{E}-03$

$3.53 \mathrm{E}-05$

$1.01 \mathrm{E}-02$

$1.84 \mathrm{E}-03$

$1.52 \mathrm{E}-05$

$1.02 E-03$

$1.62 \mathrm{E}-03$

$2.28 E-02$ 
Table 4.38. Ingestion toxicity $\left(\mathrm{m}^{3}\right.$ water) of accumulated heavy isotopes in intermediate-ratiation-level TRU wastes at a federal repository

in the year 2005 as a function of age

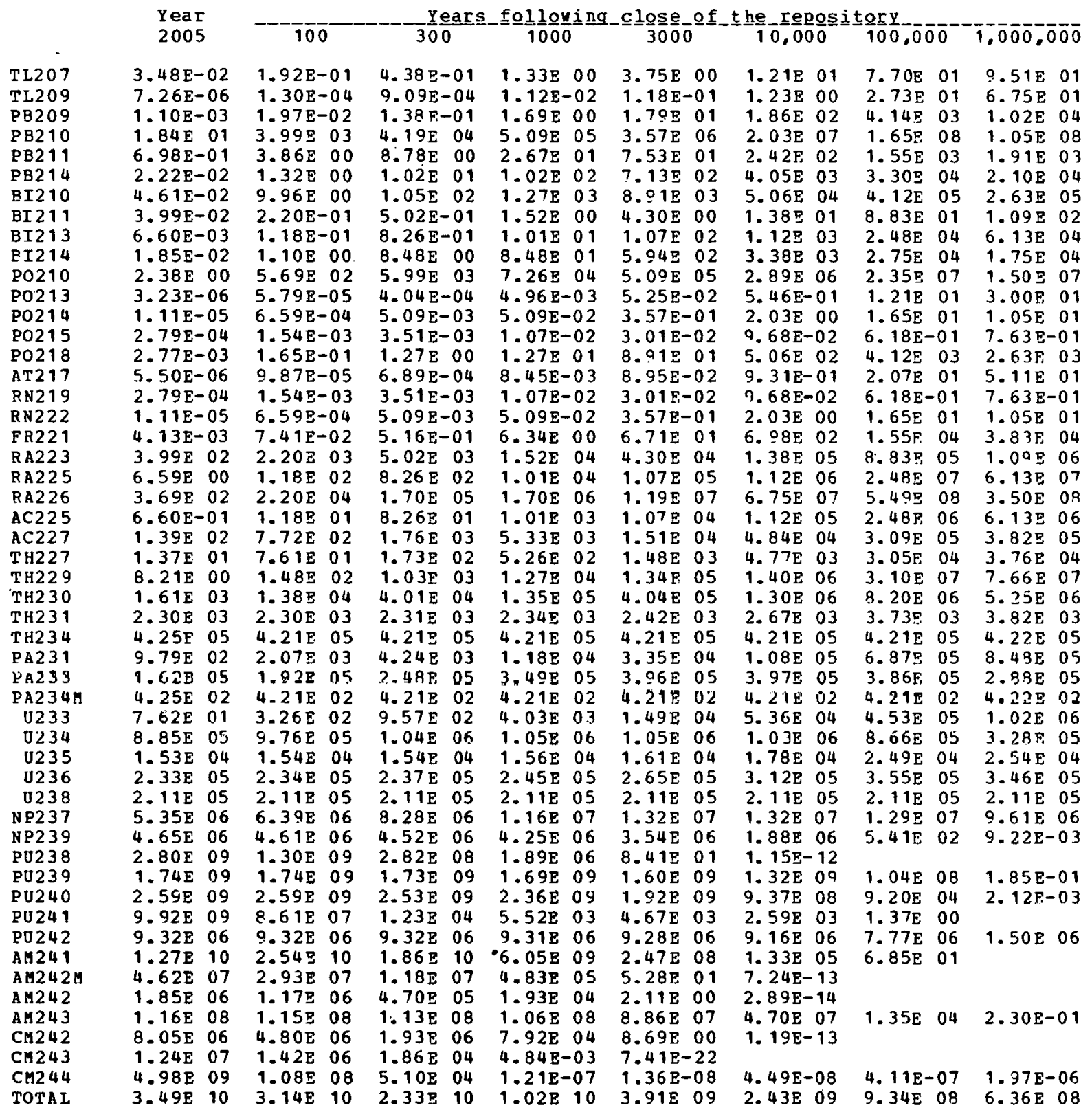


Table 4.39. Grams of accumulated fission product elements in intermediateradiation-level TRO wastes at a federal repository

in the year 2005 as a function of age

Year

2005

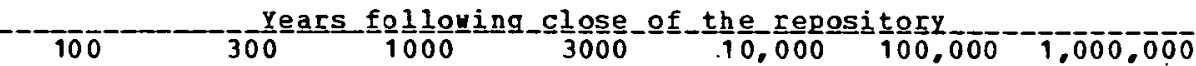

1. $14 \mathrm{E} 01$

$1.14 \mathrm{E} 01$

1. $14 \mathrm{E} 01$

$1.14 \mathrm{E} 01 \quad 1.14 \mathrm{E} 01$

1. $14 \mathrm{E} 01$

3. $38 \mathrm{E} 00$

$3.38 \mathrm{E} 00$

$3.38 \mathrm{E} 00$

$3.38 \mathrm{E} 00$

$3.38 \mathrm{E} 00$

1. 14E 01

$1.14 \mathrm{E} 01$

$1.51 E \quad 03$

$1.51 \mathrm{E} 03$

$1.51 \mathrm{E} \quad 03$

$1.51 \mathrm{E} 03$

$1.51 \mathrm{E} \quad 03$

1. $50 \mathrm{E} 03$

$9.12 \mathrm{E} 03$ S.12E 03

$5.98 \mathrm{E} 02$

5.99802

$6.02 \mathrm{E} \quad 02$

6. $13 \mathrm{E} 02$

$3.38 \mathrm{E} 00$

$3.38 \mathrm{E} \quad 00$

$2.03 \mathrm{E} 04$

$1.02 \mathrm{E} 04$

$9.29 \mathrm{E} 03$

$9.12 \mathrm{E} \quad 03$

$9.12 \mathrm{E} 03$

$1.41 \mathrm{E} 03$

1. $36 \mathrm{E} \quad 03$

$1.23 \mathrm{E} 04$

$1.23 \mathrm{E} 04$

1. $23 \mathrm{E} 04$

$9.28 \mathrm{E} \quad 03$

9. $12 \mathrm{E} 03$

$6.95 \mathrm{E} \quad 2$

$7.47 \mathrm{P} \quad 02$

$9.95 \mathrm{E} 04$

$1.10 \mathrm{E} 05$

i. 11 E 05

$9.28 \mathrm{E} \quad 03$

9. $28 \mathrm{E} 03$

$9.12 \mathrm{E} 03$

$9.12 \mathrm{E} \quad 03$

$1.30 \mathrm{E}-01$

$1.02 \mathrm{E} 00$

$2.80 \mathrm{E} 00$

$1.23 \mathrm{E} 04 \quad 1.23 \mathrm{E} 04$

1. $23 \mathrm{E} 04$

$9.28 \mathrm{E} 03$

$9.28 \mathrm{E} 03$

9.25E 04

$1.11 \mathrm{E} 05$

$1.10 \mathrm{P} \quad 05$

1. $10 \mathrm{E} 05$

$\begin{array}{llllllll}2.80 \mathrm{E} & 00 & 9.03 \mathrm{E} & 00 & 2.68 \mathrm{E} & 01 & 8.90 \mathrm{E} & 01 \\ 9.25 \mathrm{E} & 04 & 9.25 \mathrm{E} & 04 & 9.25 \mathrm{E} & 04 & 9.25 \mathrm{E} & 04\end{array}$

1.23504

1. 10505

1.23504

$9.25 \mathrm{E} 04$

$2.26 \mathrm{E} \quad 04$

$8.70 \mathrm{E} 02$

$1.03 \mathrm{E} 05$

$2.26 \mathrm{E} 04$

$5.88 \mathrm{E} 04$

$5,88 \mathrm{E} 04$

$2.20 \mathrm{E} 04$

$2.24 \mathrm{E} 04$

$1.19 \mathrm{E} 04 \quad 1.19 \mathrm{E} 04$

$1.19 \mathrm{E} 04$

$5.88 \mathrm{E} \quad 04$

2. $19 \mathrm{E} 04$

$9.25 \mathrm{E} 04$

$7.13 \mathrm{E} \quad 03$

$3.99 \mathrm{E} 04$

3.99204

1. $19 \mathrm{E} \quad 4$

$5.90 \mathrm{E} \quad 04$

5. $95 \mathrm{E} 04$

$1.63 \mathrm{E} 04$

$6.51 \mathrm{E} 04$

$9.25 \mathrm{E} \quad 04$

$8.61 \mathrm{E} 02$

$3.99 E 04$

$1.64 \mathrm{E} 03$

$\begin{array}{llll}04 & 3.99 \mathrm{E} & 04\end{array}$

1.19E 04

1. $19=04$

1. $19 \mathrm{E} 04$

$8.05 \mathrm{E} 04$

$3.99 \mathrm{E} \mathrm{O4}$

$1.19 E 04$

$1.64 \mathrm{E}$
$2.42 \mathrm{~F}$
3.33

$2.42 \mathrm{E} \quad 03$

$1.64 \mathrm{E} 03$

$1.64 \mathrm{E} 03$

1. $64 \mathrm{E} 03$

1. $70 \mathrm{E} 03$

$3.94 \mathrm{E} 04$

3. $25 \mathrm{E} 01$

$2.42 \mathrm{E} \quad 03$

$2,42 \mathrm{E} \quad 03$

$2.42 \mathrm{E} 03$

$2.42 \mathrm{E} 03$

2. $42 \mathrm{E} 03$

$2.20 \mathrm{E} \quad 3$

$\begin{array}{lllllll}3.49 \mathrm{E} & 01 & 3.49 \mathrm{E} & 01 & 3.49 \mathrm{E} & 01\end{array}$

3.49801

$1.34 \mathrm{E} 03$

$1.34 \mathrm{E} 03$

$1.33 \mathrm{E} 03$

$1.33 \mathrm{E} 03$

1. $30 \mathrm{E} 03$

3.49501

$2.54 \mathrm{E} 02$

$1.29 \mathrm{E} 04$

$2.33 \mathrm{E} 02$

$2.33 \mathrm{E} 02$

2. $33 \mathrm{E} \quad 02$

2. $33 E 02$

2. $33 \mathrm{E} 02$

1. $06 \mathrm{E} 03$

$2.42 \mathrm{E} \quad 03$

$\begin{array}{lllll}1.29 \mathrm{E} & 04 & 1.29 \mathrm{E} & 04\end{array}$

$1.29 \mathrm{E} 04$

1. 29.04

2.33E 02

$3.49 \mathrm{E} 01$

$6.50 \mathrm{E} 03 \quad 6.50 \mathrm{E} 03$

$6.50 \mathrm{E} 03$

$6.50 \mathrm{E} 03$

$6.50 \mathrm{E} 03$

6.50203

$1.32 \mathrm{E} 04$

$7.80 \mathrm{E} 02$

$2.59 \mathrm{E}-03$

2. $38 \mathrm{E}-02$

$6.63 \mathrm{E}-02$

2. 15E-01

$6.40 E-01$

2. $13 \mathrm{~F}, 00$

$6.48 \mathrm{E} 03$

$2.33 \mathrm{E} 02$

$\begin{array}{llllllll}3.69 \mathrm{E} & 04 & 3.69 \mathrm{E} & 04 & 3.69 \mathrm{E} & 04\end{array}$

3. $69 \mathrm{E} 04$

$2.12 \mathrm{E} 01$

$1.34 \mathrm{E} 04$

$5.17 \mathrm{E} 04$

$7.52 \mathrm{E} 04$

$7.78 \mathrm{E} \quad 04$

$7.78 \mathrm{E} \quad 04$

$7.78 \mathrm{E} 04$

$3.67 \mathrm{E} 04$

.

$3.33 \mathrm{E} \quad 04$

$3.33 \mathrm{E} 04$

$3.33 \mathrm{E} \cdot 04$

3. $33 \mathrm{E} 04$

$\begin{array}{lllllllll}6.54 E & 04 & 6.54 \mathrm{E} & 04 & 6.54 \mathrm{E} & 04 & 6.54 \mathrm{E} & 04\end{array}$

$6.54 \mathrm{E} 04$

$6.54 \mathrm{E} 04$

$3.09 \mathrm{E} 04 \quad 3.09 \mathrm{E} 04$

$3.09 \mathrm{E} 04 \quad 3.09 \mathrm{E} 04$

$3.09 \mathrm{E} 04$

$3.09 \mathrm{E} 04$

$1.10 \mathrm{E} 05$

$2.24 \mathrm{E} 02 \quad 7.25 \mathrm{E}-10$

$2.34 \mathrm{E} 04$

$4.27 \mathrm{E} 03$

$1.10 \mathrm{E} 05$

1.10E 05

1. $10 \mathrm{R}, 05$

1. $10 \mathrm{E} 05$

$7.80 \mathrm{E} 04$

3.51504

$7.96 \mathrm{E} 04$

$6.54 \mathrm{E} .04$

$2.30 \mathrm{E} 04$

$2.26 \mathrm{E} \quad 04$

$2.25 \mathrm{E} 04$

$2.25 E 04$

2. $25 \mathrm{E} 04$

$.09 \mathrm{E} 04$

$3.09 \mathrm{E} 04$

4. 48E 03

$4.58 \mathrm{E} \quad 03$

4. $58 \mathrm{E} \quad 03$

4. 14E 03

$4.03 E 01$

4. 15E 03

$4.15 \mathrm{E} \quad 03$

$4.15 \mathrm{E} 03$

4. 58 E 04

10E 05

$1.10 \mathrm{E} \quad 05$

3.52E 01 3.52E 01

$3.63 \mathrm{E} 00 \quad 3.63 \mathrm{E} 00$

$\mathrm{E} 0$

4. $93 \mathrm{E} 01$

4.93 $\mathrm{E} 01$

4. $15 \mathrm{E} 03$

$2.25 \mathrm{E} \quad 04$

$2.25 E 04$

$\begin{array}{lllll}3.52 \mathrm{E} & 01 & 3.52 \mathrm{E} & 01\end{array}$

$3.52 \mathrm{E} 01$

4. $93 \mathrm{E} 01$

$3.63 \mathrm{E} 00 \quad 3.63 \mathrm{E} 00$

$3.62 \mathrm{E} 00$

$3.52 \mathrm{E} 01$

$4.58 \mathrm{E} \quad 03$

4. 15.03

$4.58 \mathrm{~B}$

4. $93 \mathrm{E} 01$

$4.15 \mathrm{E} 03$

$9.85 \mathrm{E}-01$

$9.87 \mathrm{E}-01$

$9.91 \mathrm{E}-01$

9. $96 \mathrm{E}-01$

3. $62 \mathrm{E} 00$

$3.52 \mathrm{E} 01$

$4.93 \mathrm{E} 01$

$7.80 \mathrm{E}$ OS $7.80 \mathrm{E} \quad 05$

$7.80 \mathrm{E} \quad 05$

$7.80 \mathrm{E} \quad 05 \quad 7.80 \mathrm{~S} 05$

7.80505

$.62 \mathrm{E} 00$

$3.52 \mathrm{E} 01$

$3.62 \mathrm{E} 00$

7. $80 \mathrm{E} 05$

9. $99 \mathrm{E}-01$

$7.80 \mathrm{E}$ OS 
Table 4.40. Curies of accumulated fission product isotopes in intermediateradiation-level TRU wastes at a federal repository

in the year 2005 as a function of age

Year 2005

SE 79

SR 90

Y 90

ZR 93

NB $93 M$

TC 99

RU 106

RH 106

PD107

SB125

TE1 25M

SN 126

SB126M

SB1 26

I1 29

CS134

CS 135

CS137

BA $137 \mathrm{M}$

CE144

PR 144

PM147

SM151

EU154

EU155

HO166M

TOTAL
$1.04 \mathrm{E} 01$ 1. 56E 06 1. $56 \mathrm{E} 06$ 4. $94 \mathrm{E} \quad 01$ 2. $51 \mathrm{E} 01$ 3. $86 \mathrm{E} 02$ $4.57 \mathrm{E} \quad 04$ $4.57 \mathrm{E} 04$ $2.86 \mathrm{E} \quad 00$ 2. $23 \mathrm{E} 04$ $9.23 \mathrm{E} \quad 03$ 1. $59 \mathrm{E} \quad 01$ 1. 59E 01 1. $58 \mathrm{E} \quad 01$ 8. 50F-01 3. $00 \mathrm{E} \quad 05$ 7. 75E 00 2. $25 \mathrm{E} 06$ 2. 10E 06 2. 39E 04 2. 39E 04 2. $08 \mathrm{E} \quad 05$ 2. $93 E \quad 04$ 1. $11 \mathrm{E} \quad 05$ $6.29 \mathrm{E} \quad 03$ 2. $68 \mathrm{E}-02$ $8.29 \mathrm{E} 06$
Years following close of the repository

\begin{tabular}{|c|c|c|c|c|c|c|}
\hline 100 & 300 & 1000 & 3000 & 10,000 & 100,000 & 1.000 .000 \\
\hline 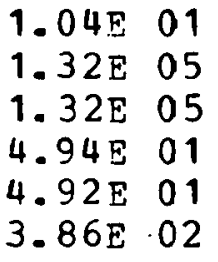 & $\begin{array}{ll}1.04 \mathrm{E} & 01 \\
9.52 \mathrm{E} & 02 \\
9.52 \mathrm{E} & 02 \\
4.94 \mathrm{E} & 01 \\
4.94 \mathrm{E} & 01 \\
3.85 \mathrm{E} & 02\end{array}$ & $\begin{array}{ll}1.03 \mathrm{E} & 01 \\
3.02 \mathrm{E}-05 \\
3.02 \mathrm{E}-05 \\
4.94 \mathrm{E} & 01 \\
4.94 \mathrm{E} & 01 \\
3.84 \mathrm{E} & 02\end{array}$ & 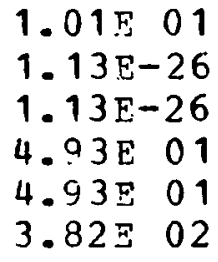 & $\begin{array}{ll}4.92 \mathrm{E} & 01 \\
4.92 \mathrm{E} & 01 \\
3.73 \mathrm{E} & 02\end{array}$ & $\begin{array}{ll}4.72 \mathrm{E} & 01 \\
4.72 \mathrm{E} & 01 \\
2.78 \mathrm{E} & 02\end{array}$ & $\begin{array}{ll}3.11 \mathrm{E} & 01 \\
3.11 \mathrm{E} & 01 \\
1.47 \mathrm{E} & 01\end{array}$ \\
\hline $\begin{array}{l}2.86 \mathrm{E} \quad 00 \\
1.58 \mathrm{E}-07 \\
6.54 \mathrm{E}-08\end{array}$ & $2.86 \mathrm{E} \quad 00$ & $2.86 \mathrm{E} \quad 00$ & $2.86 \mathrm{E} 00$ & $2.85 \mathrm{E} \quad 00$ & $2.83 \mathrm{~F}, 00$ & $2.59 \mathrm{E} \quad 00$ \\
\hline 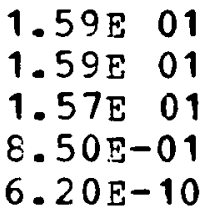 & $\begin{array}{rr}1.58 \mathrm{E} & 01 \\
1.58 \mathrm{E} & 01 \\
1.57 \mathrm{E} & 01 \\
8.50 \mathrm{E}-01\end{array}$ & $\begin{array}{rr}1.58 \mathrm{E} & 01 \\
1.58 \mathrm{E} & 01 \\
1.56 \mathrm{E} & 01 \\
8.50 \mathrm{E}-01\end{array}$ & $\begin{array}{rr}1.55 E & 01 \\
1.55 E & 01 \\
1.54 E & 01 \\
8.50 E-01\end{array}$ & $\begin{array}{rr}1.48 E & 01 \\
1.48 E & 01 \\
1.47 E & 01 \\
8.50 E-01\end{array}$ & $\begin{array}{ll}7.93 \mathrm{E} & 00 \\
7.93 \mathrm{E} & 00 \\
7.85 \mathrm{E} & 00 \\
8.47 \mathrm{E}-01\end{array}$ & $\begin{array}{l}1.55 \mathrm{E}-02 \\
1.55 \mathrm{E}-02 \\
1.53 \mathrm{E}-02 \\
8.16 \mathrm{E}-01\end{array}$ \\
\hline 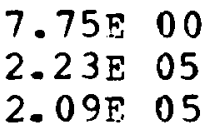 & $\begin{array}{ll}7.75 \mathrm{E} & 00 \\
2.19 \mathrm{E} & 03 \\
2.05 \mathrm{E} & 03\end{array}$ & $\begin{array}{l}7.75 E \quad 00 \\
2.08 E-04 \\
1.94 E-04\end{array}$ & $\begin{array}{l}7.74 \mathrm{E} \quad 00 \\
1.77 \mathrm{E}-24 \\
1.66 \mathrm{E}-24\end{array}$ & $7.73 \mathrm{E} \quad 00$ & $7.57 F: 00$ & $6.15 \mathrm{~F} \quad 00$ \\
\hline $\begin{array}{l}.73 E-07 \\
.32 \mathrm{E} \quad 04 \\
.46 \mathrm{E} 03 \\
.47 \mathrm{E}-13 \\
.53 \mathrm{E}-02 \\
11 \mathrm{~F} \\
.15\end{array}$ & $\begin{array}{rr}2.68 \mathrm{E} & 03 \\
2.51 \mathrm{E}-01\end{array}$ & $\begin{array}{l}1.01 \mathrm{E} \quad 01 \\
1.70 \mathrm{E}-14\end{array}$ & $.22 E-06$ & 8. 31E-05 & 2. $20 F-27$ & \\
\hline
\end{tabular}


Table 4.41. Watts of accumulated fission product isotopes in intermediateradiation-level TRU wastes at a federal repository in the year 2005 as a function of age

\begin{tabular}{|c|c|c|c|c|c|c|c|c|}
\hline & Year & \multicolumn{7}{|c|}{ 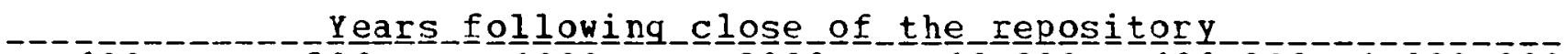 } \\
\hline & 2005 & 100 & 300 & 1000 & 3000 & 10.000 & 100,000 & $1.000,000$ \\
\hline SE 79 & 3. $94 \mathrm{E}-03$ & $3.94 E-03$ & $3.93 \mathrm{~F}-03$ & $3.90 E-03$ & $3.82 E-03$ & $3.55 \mathrm{E}-03$ & 1. $36 \mathrm{~F}-03$ & $9.225-08$ \\
\hline$S R \equiv 0$ & $2.04 \mathrm{E} 03$ & $1.73 \mathrm{E} \quad 02$ & $1.25 \mathrm{E} \quad 00$ & 3. $95 \mathrm{E}-08$ & $1.48 \mathrm{~F}-29$ & & & \\
\hline Y. 90 & $9.17 \mathrm{E} 03$ & $7.78 \mathrm{E} \quad 02$ & $5.61 \mathrm{E} 00$ & $1.78 \mathrm{E}-07$ & $6.66 \mathrm{z}-29$ & & & \\
\hline$Z R=3$ & $5.86 \mathrm{E}-03$ & $5.865-03$ & $5.86 \mathrm{e}-03$ & $5.85 \mathrm{E}-03$ & -03 & $5.83 F-03$ & $5.59 F-0.3$ & $3.69 E-03$ \\
\hline $\mathrm{NB} \quad 93 \mathrm{M}$ & 4. $46 \mathrm{E}-03$ & $8.76 \pm-03$ & $9.78 \mathrm{E}-03$ & -03 & $8.77 \mathrm{E}-03$ & $8.74 E-03$ & 8.3 & -03 \\
\hline TC 99 & $2.61 E-01$ & 2. $61 \Sigma-01$ & $2.60 E-01$ & $2.60 \mathrm{E}-01$ & $2.58 F-01$ & $2.52 \mathrm{E}-01$ & 1. $88 E-01$ & $8-03$ \\
\hline RU106 & $2.71 \mathrm{E} \quad 00$ & & & & & & & \\
\hline RH 106 & $4.81 \mathrm{E} \quad 02$ & & & & & & & \\
\hline PD107 & $2.37 E-04$ & $2.37 s-04$ & $2.37 E-04$ & $2.37 \mathrm{E}-04$ & $2.37 F-04$ & $2.37 E-04$ & $2.35 F-04$ & $2.15 E-04$ \\
\hline SB125 & $9.04 \mathrm{E} 01$ & $6.40 \mathrm{E}-10$ & & & & & & \\
\hline TE125M & 1.59E: 01 & 1. $13 \mathrm{E}-10$ & & & & & & \\
\hline SN 126 & $1.71 \mathrm{E}-02$ & 1. $71 E-02$ & $1.71 \mathrm{E}-02$ & 1. $70 E-02$ & $1.68 \mathrm{E}-02$ & 1. $60 \mathrm{E}-02$ & $8.56 P-03$ & $E-05$ \\
\hline SB126M & $1.07 \mathrm{E}-01$ & $1.07 F-01$ & $1.07 \mathrm{E}-01$ & 1. $.06 \mathrm{E}-01$ & $1.05 z-01$ & 1. $00 E-01$ & 5.3 & -04 \\
\hline SB126 & $2.05 \mathrm{~F}-01$ & $2.045-01$ & $2.04 \mathrm{~s}-01$ & $2.03 \Xi-01$ & $2.00 E-01$ & 1. $91 \mathrm{E}-01$ & $1.02 \mathrm{~F}-01$ & DE-04 \\
\hline I 120 & $5.59 E-04$ & $5.59 E-04$ & $5.59 \mathrm{~g}-04$ & $5.59 \mathrm{E}-04$ & $5.59 \mathrm{E}-04$ & $5.59 E-04$ & $5.57 \mathrm{~s}-04$ & $5.37 E-04$ \\
\hline $\operatorname{cs} 134$ & $3.18 \mathrm{~F} 03$ & $6.57 E-12$ & & & & & & \\
\hline CS 135 & $3.77 E-03$ & $3.77 E-03$ & $3.77 \mathrm{E}-03$ & $3.77 E-03$ & $3.76 s-03$ & $3.76 \mathrm{E}-0.3$ & 3. $68 \mathrm{E}-03$ & $2.99 E-0.3$ \\
\hline $\operatorname{cs} 137$ & $3.68 \mathrm{E} \quad 03$ & $3.65 \mathrm{~F} 02$ & $3.59 \mathrm{E} 00$ & $3.40 E-07$ & $2.90 E-27$ & & & \\
\hline BA1ミ7M & $8.27 F \quad 03$ & $8.20 \mathrm{E} \quad 02$ & $8.07 \mathrm{E} 00$ & 7. $.63 E-07$ & $6.51 \mathrm{~F}-27$ & & & \\
\hline CE144 & $96 \mathrm{~F}, 01$ & & & & & & & \\
\hline PR144 & $35 E: 02$ & & & & & & & \\
\hline PM 147 & $1.07 F 02$ & $3.47 \mathrm{E}-10$ & & & & & & \\
\hline SM151 & $5.10 \mathrm{E} 01$ & $2.30 \mathrm{E} 01$ & $4.67 \mathrm{E} 00$ & $1.77 \mathrm{E}-02$ & $2.12 \mathrm{E}-09$ & & & \\
\hline EU 154 & $9.09 E \quad 02$ & $1.19 \mathrm{E} \quad 01$ & $2.06 E-03$ & 1. $39 \mathrm{E}-16$ & & & & \\
\hline EU155 & $5.29 \mathrm{E} 00$ & $1.24 \Sigma-16$ & & & & & & \\
\hline Ho16t & $8 \mathrm{E}-04$ & $2.72 \mathrm{E}-04$ & $2.42 E-04$ & $1.62 \mathrm{~F}-04$ & $5.09 E-05$ & $8.93 F-07$ & $2.36 \mathrm{E}-29$ & \\
\hline TOTAL & $2 \mathrm{E} 04$ & $7 \mathrm{E} \quad 03$ & 1 & 01 & $6.03 F-01$ & 82 & 01 & $2 t$ \\
\hline
\end{tabular}


Table 4.42. Ingestion toxicity ( ${ }^{3}$ water) of accumulated fission product isotopes in intermediate-radiation-level TRU wastes at a federal repository in tile year 2005 as a function. of age

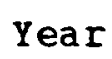
2005

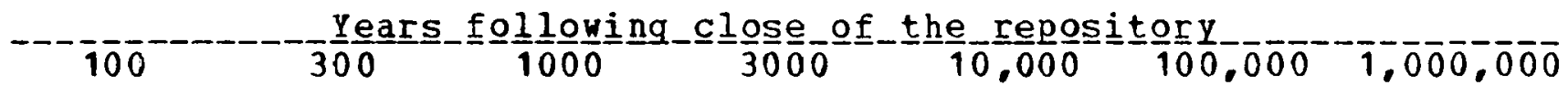

\section{$\begin{array}{lllllllll}1.04 \mathrm{E} & 01 & 1.04 \mathrm{E} & 01 & 1.03 \mathrm{E} & 01 & 1.01 \mathrm{E} & 01\end{array}$}

$\begin{array}{lllllll}4.40 \mathrm{E} & 11 & 3.17 \mathrm{E} & 09 & 1.01 \mathrm{E} & 02 & 3.77 \mathrm{E}-20\end{array}$

$6.60 \mathrm{E} \quad 09 \quad 4.76 \mathrm{E} \quad 07 \quad 1.51 \mathrm{E} \quad 00 \quad 5.66 \mathrm{E}-22$

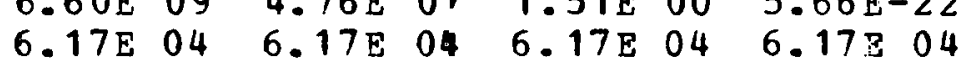

$\begin{array}{llllllll}1.23 \mathrm{E} & 05 & 1.23 \mathrm{E} & 05 & 1.23 \mathrm{E} & 05 & 1.23 \mathrm{E} & 05\end{array}$

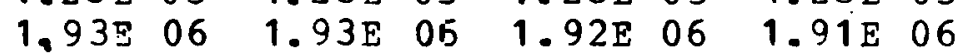

9. $35 \mathrm{E} 00$

3. $58 \mathrm{E} 00$

$2.43 E-04$

$5.19 \mathrm{E} \quad 12$

$7.79 \mathrm{E} 10$

$6.17 \mathrm{E} 04$

$6.27 \mathrm{E} 04$

$1.93 \mathrm{E} 06$

4.57E 09

$4.57 \mathrm{E} \quad 04$

$2.86 \mathrm{E} 03$

$2.23 \mathrm{E} 08$

$9.23 \mathrm{E} 07$

$1.59 \mathrm{E} 01$

$1.59 \mathrm{E} 01$

1. $58 \mathrm{E} 01$

$1.42 E 07$

$3.33 \mathrm{E} \quad 10$

$7.75 \mathrm{E} 04$

1. $12 \mathrm{E} 11$

2. 10E 06

2.39E 09

$2.39 \mathrm{E} 04$

$1.04 \mathrm{E} 09$

$\begin{array}{ll}7.32 \mathrm{E} & 07 \\ 5.54 \mathrm{E} & 09\end{array}$

$2.86 \mathrm{E} \quad 03$

1. $58 \mathrm{E}-03$

$6.54 \mathrm{E}-04$

1. $59 \mathrm{E} \quad 01$

$1.59 \mathrm{E} 01$

$1.57 \mathrm{E} \quad 01$

$1.42 \mathrm{E} \quad 07$

$6.89 \mathrm{E}-05$

$7.75 \mathrm{E} \quad 04$

$1.12 \mathrm{~F} \quad 10$

$2.09 \mathrm{E} 05$

$2.86 \mathrm{E} \quad 03$

$2.86 \mathrm{E} \quad 03$

$2.86 \mathrm{E} \quad 03$

$6.15 \mathrm{E} 04$

1. $23 \mathrm{E} 05$

$\begin{array}{llll}5.90 \mathrm{E} & 04 & 3.89 \mathrm{~F} & 04\end{array}$

1. $87 \mathrm{E} 06$

$\begin{array}{lllll}1.18 E & 05 & 7.78 \mathrm{E} & 04\end{array}$

1. $39 \mathrm{E} 06 \quad 7.33 \mathrm{E} 04$

$1.58 \mathrm{E} 01$

$1.58 \mathrm{E} 01$

$1.57 \mathrm{E} \quad 01$

$1.42 \mathrm{E} \quad 07$

1. $58 \mathrm{E} \quad 01$

$1.58 \mathrm{E} 01$

$1.56 \dot{\mathrm{E}} 01$

$7.75 \mathrm{E} \quad 04$

1. $10 \mathrm{E} 08$

$1.42 \mathrm{E} 07$

1. $55 \mathrm{E} 01$

$1.55 \mathrm{E} \quad 01$

$1.54 \Sigma 01$

$1.42 \mathrm{E} 07$

$2.85 \mathrm{E} 03$

$2.83 \mathrm{E} \quad 03 \quad 2.59 \mathrm{E} \quad 03$

g

$3.37 E-03$

3.30807

$7.28 \mathrm{E} \quad 07$

$3.14 \mathrm{E} 07 \quad 7.34 \mathrm{E}-10$

$2.68 \mathrm{E}-02$

7. $34 \mathrm{E}-10$
$2.53 \mathrm{E}-02$

$2.05 \mathrm{E} 03$

$7.75 \mathrm{E} 04$

$7.74 \mathrm{E} 04$

$1.04 \mathrm{E} 01 \quad 8.85 \mathrm{E}-20$

1. $48 \mathrm{E} 01$

7. $.3 \mathrm{E} 00 \quad 1.55 \mathrm{E}-02$

$\begin{array}{lllll}1.48 \mathrm{E} & 01 & 7.93 \mathrm{E} & 00 & 1.55 \mathrm{E}-02\end{array}$

$\begin{array}{lllll}1.47 \mathrm{E} & 01 & 7.85 \mathrm{E} & 00 & 1.53 \mathrm{E}-0 ?\end{array}$

$\begin{array}{llllll}1.42 \mathrm{E} & 07 & 1.41 \mathrm{E} & 07 & 1.36 \mathrm{E} & 07\end{array}$

$\begin{array}{llllll}7.73 \mathrm{E} & 04 & 7.57 \mathrm{E} & 04 & 6.15 \mathrm{E} & 04\end{array}$

$6.70 \mathrm{E} 06 \quad 2.54 \mathrm{E} 04 \quad 3.05 \mathrm{E}-03$

$1.26 \mathrm{E} 04 \quad 8.48 \mathrm{E}-10$

EU155

H0166M

TOTAL
$5.43 \mathrm{E} \quad 12$
$3.35 \mathrm{E} \mathrm{G9}$
$2.25 E-C 2$
1. $50 \mathrm{E}-02$

$1.64 \mathrm{E} 07$
$4.74 \mathrm{E}-03$

$1.63 \mathrm{E} 07$
8. $31 \mathrm{E}-05$

$1.63 \mathrm{E} 07$
2. $20 E-27$

$\begin{array}{lllll}1.58 \mathrm{E} & 07 & 1.39 \mathrm{E} & 07\end{array}$ 
made of paper, cloth, wood, plastic, rubber, glass, ceramic, and metal, as well as immobilized salts and sludges that arise in the treatment of liquid waste streams and filters from cleanup of off-gas. The transuranium-element content of these wastes ranges from trace amounts to several grams per cubic foot, and averages about $0.25 \mathrm{~g} / \mathrm{ft}^{3}$. The densities of the uncompacted wastes vary from about $2 \mathrm{lb} / \mathrm{ft}^{3}$ to as much as $200 \mathrm{Ib} / \mathrm{ft}^{3}$. About one-half to two-thirds of these wastes (by volume) are combustible and can be reduced via incineration by factors of about 50 and 20 in volume and weight, respectively. About one-half to three-fourths of the wastes (by volume) can be reduced in volume by factors of 2 to 10 through compaction.

A survey of operations at ERDA laboratories and production facilities indicated that future large plants will generate about 10,000, 20,000, and $4000 \mathrm{ft}^{3}$ of uncompacted low-level TRU waste per ton of plutonium or ${ }^{23} \mathrm{U}$ processed in fuel preparation, fabrication, and reprocessing, respectively. ${ }^{26}$ Thus, an average plutonium or ${ }^{233} \mathrm{U}$ concentration in the waste of $0.25 \mathrm{~g} / \mathrm{ft}^{3}$ represents fuel losses of $0.25 \%$ in preparation, $0.5 \%$ in fabrication, and $0.1 \%$ in reprocessing.

Table 4.43 presents projections of low-level plutonium TRU wastes' assuming they are shipped and accumulated at a repository five years following their generation. 'The isotopic composition of the plutonium is taken as $1 \%{ }^{238} \mathrm{Pu}, 59 \%{ }^{239} \mathrm{Pu}, 24 \%{ }^{240} \mathrm{Pu}, 12 \%{ }^{241} \mathrm{Pu}$, and $4 \%{ }^{242} \mathrm{Pu} .{ }^{1}$ It is assumed that the wastes are compacted or otherwise reduced in volume by factors of 3 to 10 according to the following schedule:

\begin{tabular}{cc}
$\begin{array}{c}\text { Calendar } \\
\text { year }\end{array}$ & $\begin{array}{c}\text { Compaction } \\
\text { factor }\end{array}$ \\
\hline $1976-1981$ & 3 \\
$1982-1083$ & 4 \\
$1984-1.985$ & 5 \\
$1986-1987$ & 7.5 \\
$1988-2000$ & 10
\end{tabular}

Low-level TRU wastes are packaged in Type 17C, 55-gal steel drums and shipped in ATMX-600 railcars. ${ }^{28}$ Each shipment contains $1000 \mathrm{ft}^{3}$ of waste. 
Table 4.43. Projected annual accumulation of low-level plutonium transuranic waste at a federal repository

\begin{tabular}{|c|c|c|c|c|c|}
\hline Year & $\begin{array}{l}\text { \# * } \\
\text { Vclume } \\
\text { (Thousands } \\
\left.\text { of } \mathrm{m}^{3}\right)\end{array}$ & $\begin{array}{l}\text { Annual A } \\
\text { Actinide } \\
\text { Mass } \\
\text { (MT) }\end{array}$ & $\begin{array}{l}\text { dition } \\
\text { Radio- } \\
\text { activity } \\
\text { (HCi) }\end{array}$ & $\begin{array}{l}\text { * * * } \\
\text { Thermal } \\
\text { Pouer } \\
\text { (kW) }\end{array}$ & $\begin{array}{c}\text { Annual } \\
\text { Nunber } \\
\text { of } \\
\text { Shi ग़ents }\end{array}$ \\
\hline $\begin{array}{l}1986 \\
1987 \\
1988 \\
1989 \\
1990 \\
1991 \\
1992 \\
1993 \\
1994 \\
1995 \\
1996 \\
1997 \\
1998 \\
1999 \\
2000 \\
2001 \\
2002 \\
2003 \\
2004 \\
2005\end{array}$ & $\begin{array}{r}0.1 \\
1.0 \\
2.0 \\
3.4 \\
3.7 \\
2.6 \\
3.2 \\
2.1 \\
2.9 \\
4.5 \\
5.9 \\
6.6 \\
6.7 \\
6.1 \\
7.2 \\
8.8 \\
9.9 \\
10.9 \\
11.6 \\
11.7\end{array}$ & $\begin{array}{l}0.003 \\
0.035 \\
0.069 \\
0.149 \\
0.162 \\
0.171 \\
0.210 \\
0.190 \\
0.259 \\
0.396 \\
0.523 \\
0.585 \\
0.590 \\
0.535 \\
0.633 \\
0.780 \\
0.877 \\
0.962 \\
1.021 \\
1.032\end{array}$ & $\begin{array}{r}0.0 \\
0.3 \\
0.7 \\
1.4 \\
1.6 \\
1.7 \\
2.0 \\
1.8 \\
2.5 \\
3.9 \\
5.1 \\
5.7 \\
5.8 \\
5.2 \\
6.2 \\
7.6 \\
8.5 \\
9.4 \\
9.9 \\
10.0\end{array}$ & $\begin{array}{l}0.0 \\
0.4 \\
0.8 \\
1.7 \\
1.9 \\
2.0 \\
2.5 \\
2.2 \\
3.0 \\
4.6 \\
6.1 \\
6.8 \\
6.9 \\
6.2 \\
7.4 \\
9.1 \\
10.2 \\
11.2 \\
11.9 \\
12.0\end{array}$ & $\begin{array}{r}4 \\
35 \\
59 \\
119 \\
129 \\
71 \\
112 \\
76 \\
104 \\
158 \\
209 \\
234 \\
236 \\
214 \\
253 \\
312 \\
351 \\
3: 85 \\
408 \\
413\end{array}$ \\
\hline
\end{tabular}

\begin{tabular}{|c|c|c|c|c|c|c|c|}
\hline $\begin{array}{l}\text { Volume } \\
\text { (Millions } \\
\left.\text { of } \mathrm{m}^{3}\right)\end{array}$ & $\begin{array}{c}\text { Actinide } \\
\text { Mass } \\
\text { (MT) }\end{array}$ & $\begin{array}{l}\text { Radio- } \\
\text { activity } \\
\text { (MCi) }\end{array}$ & $\begin{array}{c}\text { Thermal } \\
\text { Power } \\
\text { (kw) }\end{array}$ & $\begin{array}{l}\text { Haz } \\
\text { Met } \\
\text { Air }\end{array}$ & $\begin{array}{l}\text { ard, } \\
\text { ers }\end{array}$ & $\begin{array}{l}\text { Cubic } \\
\text { at RCG } \\
\text { Water }\end{array}$ & \\
\hline $\begin{array}{l}0.000 \\
0.001 \\
0.003 \\
0.006 \\
0.010 \\
0.013 \\
0.016 \\
0.018 \\
0.021 \\
0.025 \\
0.031 \\
0.038 \\
0.045 \\
0.051 \\
0.058 \\
0.067 \\
0.077 \\
0.088 \\
0.099 \\
0.111\end{array}$ & $\begin{array}{l}0.00 \\
0.04 \\
0.11 \\
0.26 \\
0.42 \\
0.59 \\
0.80 \\
0.99 \\
1.25 \\
1.64 \\
2.17 \\
2.75 \\
3.34 \\
3.88 \\
4.51 \\
5.29 \\
6.17 \\
7.13 \\
8.15 \\
9.18\end{array}$ & $\begin{array}{l}0.0 \\
0.4 \\
1.0 \\
2.4 \\
3.9 \\
5.4 \\
7.2 \\
8.8 \\
10.9 \\
14.3 \\
18.8 \\
23.7 \\
28.4 \\
32.4 \\
37.2 \\
43.2 \\
49.9 \\
57.1 \\
64.6 \\
71.9\end{array}$ & $\begin{array}{r}0.0 \\
0.4 \\
1.3 \\
3.1 \\
5.0 \\
7.2 \\
9.9 \\
12.4 \\
15.8 \\
20.9 \\
27.6 \\
35.2 \\
43.1 \\
50.6 \\
59.3 \\
70.0 \\
82.0 \\
95.3 \\
109.6 \\
124.3\end{array}$ & $\begin{array}{l}2.4 \mathrm{E} \\
2.8 \mathrm{E} \\
7.9 \mathrm{E} \\
1.9 \mathrm{E} \\
3.1 \mathrm{E} \\
4.3 \mathrm{E} \\
5.8 \mathrm{E} \\
7.1 \mathrm{E} \\
9.0 \mathrm{E} \\
1.2 \mathrm{E} \\
1.6 \mathrm{E} \\
2.0 \mathrm{E} \\
2.4 \mathrm{E} \\
2.8 \mathrm{E} \\
3.2 \mathrm{E} \\
3.7 \mathrm{E} \\
4.3 \mathrm{E} \\
5.0 \mathrm{E} \\
5.7 \mathrm{E} \\
6.4 \mathrm{E}\end{array}$ & $\begin{array}{l}16 \\
17 \\
17 \\
18 \\
18 \\
18 \\
18 \\
18 \\
18 \\
19 \\
19 \\
19 \\
19 \\
19 \\
19 \\
19 \\
19 \\
19 \\
19 \\
19\end{array}$ & $\begin{array}{l}4.0 \mathrm{E} \\
4.6 \mathrm{E} \\
1.3 \mathrm{E} \\
3.1 \mathrm{E} \\
5.1 \mathrm{E} \\
7.2 \mathrm{E} \\
9.8 \mathrm{E} \\
1.2 \mathrm{E} \\
1.5 \mathrm{E} \\
2.0 \mathrm{E} \\
2.7 \mathrm{E} \\
3.4 \mathrm{E} \\
4.1 \mathrm{E} \\
4.8 \mathrm{E} \\
5.6 \mathrm{E} \\
6.6 \mathrm{E} \\
7.7 \mathrm{E} \\
8.9 \mathrm{E} \\
1.0 \mathrm{E} \\
1.2 \mathrm{E}\end{array}$ & $\begin{array}{l}08 \\
09 \\
10 \\
10 \\
10 \\
10 \\
10 \\
11 \\
11 \\
11 \\
11 \\
11 \\
11 \\
11 \\
11 \\
11 \\
11 \\
11 \\
12 \\
12\end{array}$ \\
\hline $\begin{array}{l}0.111 \\
0.111 \\
0.111 \\
0.111 \\
0.111 \\
0.111 \\
0.111 \\
0.111 \\
0.111\end{array}$ & $\begin{array}{l}9.18 \\
9.18 \\
9.18 \\
9.18 \\
9.18 \\
9.18 \\
9.18 \\
9.18 \\
9.18\end{array}$ & $\begin{array}{l}5.3 \\
3.3 \\
1.5 \\
0.7 \\
0.4 \\
0.2 \\
0.0 \\
0.0 \\
0.0\end{array}$ & $\begin{array}{r}155.5 \\
108.8 \\
49.6 \\
21.7 \\
13.3 \\
5.2 \\
0.8 \\
0.2 \\
0.2\end{array}$ & $\begin{array}{l}3.9 \mathrm{E} \\
2.7 \mathrm{E} \\
1.7 \mathrm{E} \\
1.1 \mathrm{E} \\
7.1 \mathrm{E} \\
2.8 \mathrm{E} \\
3.7 \mathrm{E} \\
5.3 \mathrm{E} \\
4.0 \mathrm{E}\end{array}$ & $\begin{array}{l}19 \\
19 \\
19 \\
19 \\
18 \\
18 \\
17 \\
16 \\
16\end{array}$ & $\begin{array}{l}1.1 \mathrm{E} \\
7.8 \mathrm{E} \\
3.5 \mathrm{E} \\
1.4 \mathrm{E} \\
8.7 \mathrm{E} \\
3.9 \mathrm{E} \\
1.8 \mathrm{E} \\
1.6 \mathrm{E} \\
5.3 \mathrm{E}\end{array}$ & $\begin{array}{l}12 \\
11 \\
11 \\
11 \\
10 \\
10 \\
10 \\
10 \\
09\end{array}$ \\
\hline
\end{tabular}

TIME AFTER SHOTDONN, YEARS

100
300
1000
3000
10000
30000
100000
300000
1000000


Shipment and accumulations of low-level ${ }^{23} \mathrm{U}$ wastes from the HTGR fuel cycle are projected in Table 4.44 using the same assumptions as were adopted for the low-level plutonium TRU wastes. The isotopic composition of the HTGR uranium is taken as $0.041 \%{ }^{232} \mathrm{U}, 59.7 \%{ }^{23} \mathrm{U}$, $26.2 \%{ }^{234} \mathrm{U}, 7.9 \%{ }^{235} \mathrm{U}$, and $6.1 \%{ }^{236} \mathrm{U} .^{1}$

The accumulated mass, radioactivity, thermal power, and ingestion toxicity of the low-level plutonium and ${ }^{233} \mathrm{U}$ wastes as a function of time following the year 2005 are presented in Tables 4.45 through 4.48 and 4.49 through 4.52 , respectively.

\subsection{Noble Gases}

The noble-gas fission products consist principally of stable and short-lived isotopes of krypton and xenon. Typically, from 5 to $6 \mathrm{~kg}$ are present in each ton of spent fuel from LWRs and LMFBRs and, while xenon comprises about $95 \%$ of the weight of the mixture, the only radioisotope remaining after 150 days decay is $10.8-\mathrm{y}{ }^{85} \mathrm{Kr}$. These gases are currently discharged to the atmosphere following interim holdup for decay. Over 99\% of them are released at reprocessing plants, and the remainder are discharged at power stations as a consequence of their leakage through defective fuel cladding. Although off-site radiation exposures have been small compared with current guidelines for population exposures, the noble gases will probably be recovered in future reprocessing plants.

Several processes are under development for recovery of these gases from plant off-gas streams. ${ }^{29}$ Initially, it is likely that the mixed gases will be collected under high pressure in gas cylinders and then shipped to a large remote site and stored under conditions that will promote long-term integrity of the cylinders.

The projected accumulation of noble-gas fission products is presented in Table 4.53. It is assumed that the mixed krypton and xenon isotopes are packaged in standard 50-liter gas cylinders at a pressure of 2200 psig, stored for 1 year at the reprocessing plant, 
Table 4.44. Projected annual accumulations of low-level 0-233 wastes at a federal repository

\begin{tabular}{|c|c|c|c|c|c|}
\hline Year & $\begin{array}{l}\text { * * } \\
\text { Volume } \\
\text { (Thousands } \\
\text { of } \mathrm{m} \text { 3) }\end{array}$ & $\begin{array}{c}\text { Annual A } \\
\text { Actinide } \\
\text { Mass } \\
\text { (kg) }\end{array}$ & $\begin{array}{l}\text { daition } \\
\text { Radio- } \\
\text { activity } \\
\text { (Curies) }\end{array}$ & $\begin{array}{l}\text { * * } \\
\text { Thermal } \\
\text { Pover } \\
\text { (Hatts) }\end{array}$ & $\begin{array}{c}\text { Anrual } \\
\text { Nugber } \\
\text { of } \\
\text { Shipments }\end{array}$ \\
\hline
\end{tabular}

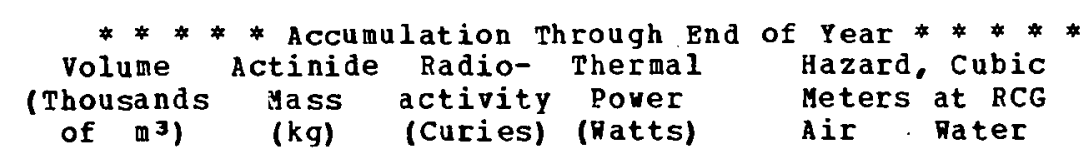

$\begin{array}{lllll}1985 & 0.002 & 0.0 & 2.8 & 0 \\ 1986 & 0.015 & 0.4 & 26.9 & 1 \\ 1987 & 0.021 & 0.7 & 49.2 & 1 \\ 1988 & 0.020 & 0.7 & 48.5 & 1 \\ 1989 & 0.016 & 0.7 & 47.7 & 1 \\ 1990 & 0.017 & 0.7 & 49.5 & 1 \\ 1991 & 0.011 & 0.8 & 50.5 & 2 \\ 1992 & 0.011 & 0.8 & 51.1 & 2 \\ 1993 & 0.009 & 0.8 & 51.0 & 2 \\ 1994 & 0.009 & 0.8 & 50.8 & 2 \\ 1995 & 0.009 & 0.8 & 51.1 & 2 \\ 1996 & 0.009 & 0.8 & 51.2 & 2 \\ 1997 & 0.009 & 0.8 & 51.2 & 2 \\ 1998 & 0.009 & 0.8 & 50.5 & 2 \\ 1999 & 0.008 & 0.7 & 49.2 & 1 \\ 2000 & 0.008 & 0.7 & 47.8 & 1 \\ 2001 & 0.008 & 0.7 & 46.5 & 1 \\ 2002 & 0.008 & 0.7 & 45.1 & 1 \\ 2003 & 0.009 & 0.8 & 52.3 & 2 \\ 2004 & 0.022 & 2.0 & 132.0 & 4 \\ 2005 & 0.048 & 4.2 & 283.5 & 8\end{array}$

0.00

0.02

0.04

0.06

0.07

0.09

0.10

0.11

0.12

0.13

0.14

0.15

0.16

0.17

0.17

0.18

0.19

0.20

0.21

0.21

0.28

$\begin{array}{rr}0 & 3 \\ 0 & 30 \\ 1 & 80 \\ 2 & 130 \\ 3 & 180 \\ 3 & 232 \\ 4 & 285 \\ 5 & 338 \\ 6 & 391 \\ 6 & 444 \\ 7 & 497 \\ 8 & 550 \\ 9 & 602 \\ 9 & 653 \\ 10 & 703 \\ 11 & 751 \\ 12 & 796 \\ 12 & 840 \\ 13 & 891 \\ 15 & 1021 \\ 19 & 1304\end{array}$

1. $2 \mathrm{E} 12$

3. 13 E 13 E 06

$\begin{array}{llll}5.4 \mathrm{E} & 13 & 6.7 \mathrm{E} & 06 \\ & 1.1 \mathrm{E} & 07\end{array}$

$\begin{array}{llll}7.5 \mathrm{E} & 13 & 1.5 \mathrm{E} & 07\end{array}$

$9.6 \mathrm{E} 13 \quad 2.0 \mathrm{E} 07$

$\begin{array}{llll}1.2 \mathrm{E} & 14 & 2.4 \mathrm{E} & 07\end{array}$

$\begin{array}{llll}1.4 \mathrm{E} & 14 & 2.9 \mathrm{E} & 07\end{array}$

1.6 E $14 \quad 3.3 \mathrm{E} 07$

$\begin{array}{lllll}1.9 \mathrm{E} & 14 & 3.8 \mathrm{E} & 07\end{array}$

$\begin{array}{llll}2.1 \mathrm{E} & 14 & 4.2 \mathrm{E} & 07\end{array}$

2. $3 \mathrm{E} 14$ 4.7E 07

$\begin{array}{llll}2.5 \mathrm{E} & 14 & 5.1 \mathrm{E} & 07 \\ 2.7 \mathrm{E} & 14 & 5.6 \mathrm{E} & 07\end{array}$

$\begin{array}{llll}2.7 \mathrm{E} & 14 & 5.6 \mathrm{E} & 07 \\ 2.98 & 14 & 6.0 \mathrm{E} & 07\end{array}$

$\begin{array}{lllll}2.9 E & 14 & 6.0 \mathrm{E} & 07 \\ 3.1 \mathrm{E} & 14 & 6.4 \mathrm{E} & 07\end{array}$

$\begin{array}{lllll}3.1 \mathrm{E} & 14 & 6.4 \mathrm{E} & 07 \\ 3.3 \mathrm{E} & 14 & 6.8 \mathrm{E} & 07\end{array}$

$\begin{array}{lllll}3.5 \mathrm{E} & 14 & 7.2 \mathrm{E} & 07\end{array}$

$3.7 \mathrm{E} 14 \quad 7.6 \mathrm{E} \quad 07$

4. 3E $14 \quad 8.7 E 07$

$\begin{array}{lllll}4.3 E & 14 & 8.7 E & 07 \\ 5.4 E & 14 & & 1.1 E & 08\end{array}$

TIME AFTER SHOTDOHN, YEARS

$\begin{array}{llr}0.28 & 19 & 613 \\ 0.28 & 19 & 233 \\ 0.28 & 19 & 219 \\ 0.28 & 19 & 356 \\ 0.28 & 19 & 674 \\ 0.28 & 19 & 939 \\ 0.28 & 19 & 841 \\ 0.28 & 19 & 460 \\ 0.28 & 19 & 44\end{array}$

$\begin{array}{llll}2.8 \mathrm{E} & 14 & 5.3 \mathrm{E} & 07 \\ 2.0 \mathrm{E} & 14 & 2.6 \mathrm{E} & 07 \\ 5.1 \mathrm{E} & 14 & 5.4 \mathrm{E} & 07 \\ 1.4 \mathrm{E} & 15 & 1.5 \mathrm{E} & 08 \\ 3.3 \mathrm{E} & 15 & 4.0 \mathrm{E} & 08 \\ 4.8 \mathrm{E} & 15 & 7.5 \mathrm{E} & 08 \\ 3.9 \mathrm{E} & 15 & 1.1 \mathrm{E} & 09 \\ 1.8 \mathrm{E} & 15 & 9.0 \mathrm{E} & 08 \\ 1.2 \mathrm{E} & 14 & 1.3 \mathrm{E} & 08\end{array}$ 
Table 4.45. Grams of a ccumulated heavy elements in low-level plutonium TRU wastes at a federal repository

in the year 2005 as a function of age

\begin{tabular}{|c|c|c|c|c|c|c|c|c|}
\hline & Year & & Y & Eo 1 1은 & cl一요으. & $\underline{h} \underline{e} \_\underline{r} \underline{e}$ & & \\
\hline & 2005 & 100 & 300 & 1000 & 3000 & 10,000 & 100,000 & $1,000,000$ \\
\hline & $1 \mathrm{E} \quad 02$ & $04 \mathrm{E}$ & $01 \geq 04$ & $2.23 \mathrm{E} 04$ & $3.69 \mathrm{E} 04$ & $6.61 \mathrm{E} 04$ & $1.45 \mathrm{E} 05$ & 1.935 \\
\hline & $65-16$ & -13 & $3 E-12$ & $E-11$ & $g-10$ & $E-0$ & $E-08$ & $E-0 B$ \\
\hline & $8 E-09$ & 3 & $\mathrm{~F}-\mathrm{C}$ & -02 & 00 & $94 \mathrm{E} \quad 0$ & $4=03$ & E 04 \\
\hline & -11 & 6. & $E-C$ & 5. & $3.05 E-01$ & $1.40 \mathrm{E} \quad 01$ & 4.635 .03 & E 05 \\
\hline & -10 & -07 & $E-06$ & -04 & 03 & $\mathrm{E}-03$ & $5-02$ & -02 \\
\hline & $E-20$ & -17 & $E-15$ & $E-14$ & $5.25 E-13$ & 6.2 & $5 E-10$ & -10 \\
\hline & $0 E-11$ & $8-08$ & $7 E-07$ & $E-06$ & $3.84 \mathrm{E}-05$ & $3 E-04$ & $0 E-03$ & -04 \\
\hline & $E-16$ & -13 & $3 E-11$ & -10 & $4.82 E-09$ & $E-0 R$ & $+2-06$ & $E-06$ \\
\hline & -06 & -0 & $E-02$ & -01 & $8 E 00$ & 3. & $\mathrm{E} \quad 02$ & 01 \\
\hline & $6 E-10$ & $2-07$ & $E-06$ & $E-05$ & $4.24 E-04$ & $1 E-03$ & $4 \Sigma-01$ & $7 E-01$ \\
\hline & 9. $E-01$ & 00 & $5 F_{1} \quad 01$ & 02 & $7.36 \mathrm{E} \quad 02$ & $2.61 \mathrm{E} \quad 0.3$ & 504 & $57 \Xi 04$ \\
\hline & $E-04$ & $E-03$ & $3 E-02$ & $E-01$ & $6.63 E-01$ & $6.21 \mathrm{E} \quad 00$ & $8 \pm 02$ & $2.40 \mathrm{E} \quad 02$ \\
\hline せ & $4 \mathrm{E} 04$ & $9.35 \mathrm{E} \quad 04$ & $7 \mathrm{E} \quad 05$ & $5 E \quad 05$ & 1. $10 \mathrm{E} \quad 06$ & $2.81 E 06$ & $3 \mathrm{E} 06$ & $7.80 \mathrm{E} \quad 06$ \\
\hline & $7 \mathrm{E} 03$ & $5 \mathrm{E} \quad 05$ & $2 \Xi 05$ & F 05 & $1.07 \mathrm{E} \quad 06$ & $1.08 \pm 05$ & $5 \mathrm{E} O \mathrm{~F}$ & $7.84 \mathrm{~F}, 05$ \\
\hline & $4 E \quad 06$ & $7 . \subseteq 9 E \quad 06$ & $8=06$ & $2 E 06$ & $6.96 \mathrm{E} 06$ & $5.23 \mathrm{E} 06$ & $6.22 \mathrm{E} 05$ & $5.90 E: 04$ \\
\hline & $6 \mathrm{E} 05$ & C.49E:05 & $3 E \quad 05$ & E 05 & $9.20 E \quad 03$ & $E-01$ & & \\
\hline & $18 E 06$ & $9.18 \mathrm{~F} \quad 06$ & $9.18 E \quad 06$ & $9.18 \mathrm{E} \quad 06$ & $9.18 \mathrm{E} 06$ & $9.18 \mathrm{E} 06$ & $9.18 \mathrm{E} \quad 06$ & $9.19 \mathrm{E} 06$ \\
\hline
\end{tabular}


Table 4.46. Curies of accumulated heavy isotopes in low-level plutonium $T R U$ wastes at a federal repository in the year 2005 as a function of aqe

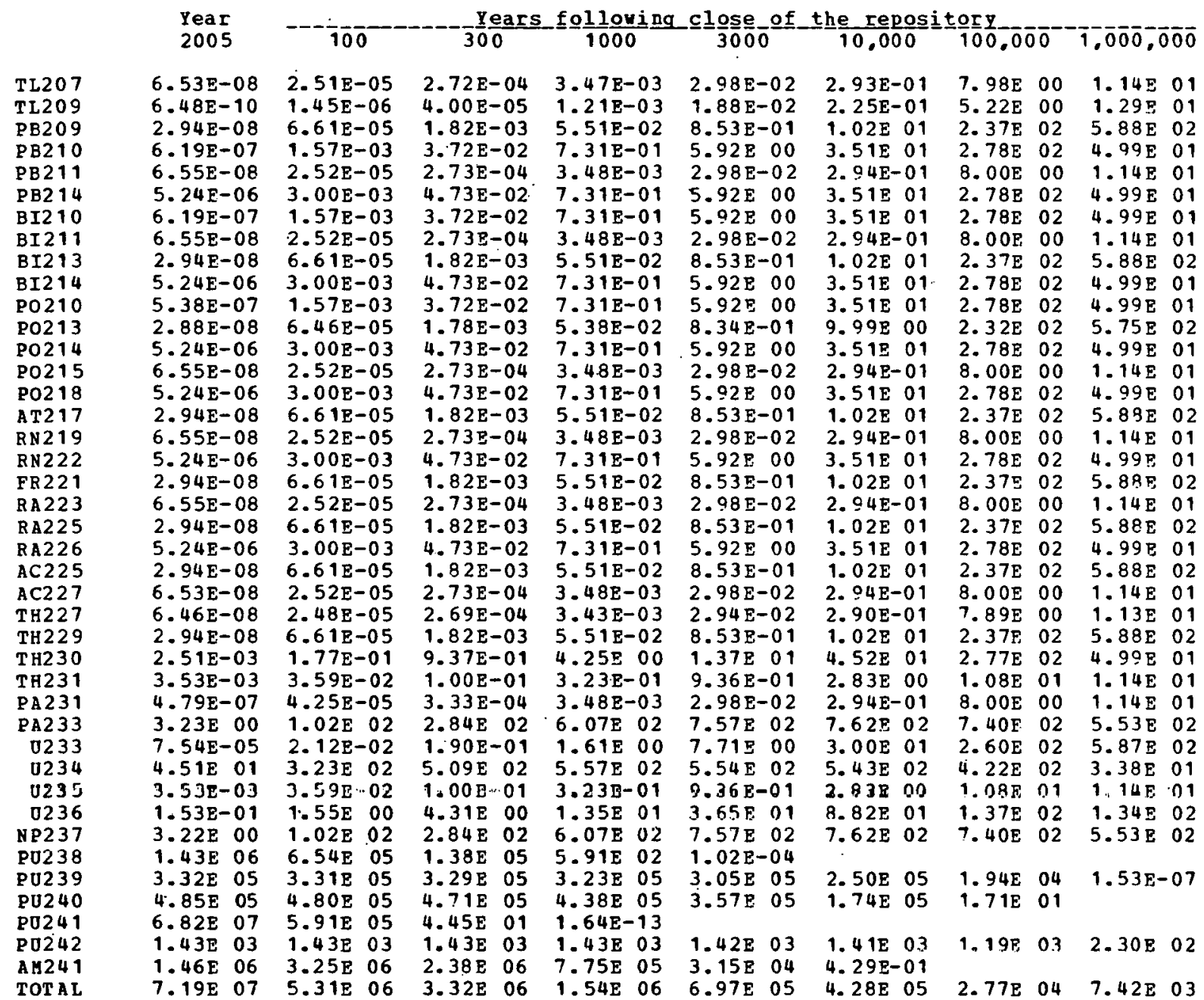


Table 4.47. Watts of accumulated heary isotopes in low-level plutonium TRU wastes at a federal repository in the year 2005 as a function of age

\begin{tabular}{|c|c|c|c|c|c|c|c|c|}
\hline & I & & & -10 & $\bar{n}$ & 470 & & \\
\hline & 2005 & 100 & 300 & 1000 & 3000 & 10.000 & 100,000 & 1.000 .000 \\
\hline 207 & $.97 \mathrm{E}-10$ & $.395-00$ & $22 E-07$ & $1.05 E-05$ & $=.00 E-05$ & $8.87 \mathrm{e}-04$ & $2.41 \mathrm{E}-02$ & $3.44 E-1$ \\
\hline & & & & & & $3.67 \mathrm{E}-03$ & $8.54 E-02$ & $8-0$ \\
\hline 82 & 11 & 08 & & 5 & $9.81 \mathrm{E}-04$ & 1. $17 \mathrm{E}-02$ & $73 E-01$ & $76 E-01$ \\
\hline & -11 & & .06 & & $46 E-04$ & 1. $46 \mathrm{E}-03$ & 1. $15 \mathrm{E}-02$ & $07 \mathrm{E}-03$ \\
\hline & 10 & & -07 & & $98 \mathrm{E}-05$ & $9.84 \mathrm{E}-04$ & $2.67 \mathrm{E}-02$ & $81 \mathrm{E}-02$ \\
\hline & & & $.15 E-04$ & $8 E-03$ & $1.44 \mathrm{E}-02$ & $8.56 \mathrm{E}-02$ & $6.77 \mathrm{E}-01$ & $22 \mathrm{E}-01$ \\
\hline I2 & & & $9.80 \mathrm{E}-05$ & & $1.56 \mathrm{E}-02$ & $9.24 \mathrm{E}-02$ & & $1 \mathrm{E}-0$ \\
\hline I 2 & & 7 & & & & 1.15 & 3. $12 E-01$ & $4.44 \mathrm{E}-01$ \\
\hline & -10 & -07 & $2 \mathrm{E}-05$ & -04 & $5.24 E-03$ & $6.28 \mathrm{E}-02$ & $1.46 \mathrm{E} \quad 00$ & IE 00 \\
\hline & & & & & & 4.89 & $3.87 \mathrm{E} \quad 00$ & $5 E-01$ \\
\hline 02 & 08 & 4.9 & $E-03$ & 2 & $1.86 E-01$ & $1.11 \mathrm{E} 00$ & $8.75 \mathrm{E} 00$ & $1.57 \mathrm{E} 00$ \\
\hline 02 & 09 & $3.2>>$ & $8.82 \mathrm{E}-05$ & 2. & $4.14 \mathrm{E}-02$ & 4. $96 \mathrm{E}-01$ & $1.15 \mathrm{E} \quad 01$ & 36E 01 \\
\hline 02 & & & $6 E-03$ & & $2.70 E-01$ & $1.60 \mathrm{E} \quad 00$ & $1.27 \mathrm{E} \quad 01$ & $2.27 \mathrm{E} \quad 00$ \\
\hline & & & 05 & 4 & $1.31 E-03$ & 1. $2.9 E-02$ & $3.50 E-01$ & $5.00 \mathrm{E}-01$ \\
\hline 02 & & 1.0 & $1 E-03$ & 2 & $2.14 E-01$ & $1.27 \mathrm{E} \quad 00$ & $1.01 \mathrm{E} \quad 01$ & $1 \mathrm{E} \quad 00$ \\
\hline $\mathrm{T} 2$ & 19 & & $1=-1$ & & $57 \varepsilon-02$ & 4. 2 & $9.95 \mathrm{E} \quad 00$ & $46 \mathrm{E} 01$ \\
\hline & & & 1. & 4 & $E-03$ & 1.1 & $3.24 E-01$ & $4.62 \mathrm{E}-01$ \\
\hline N2 & 07 & 9.7 & 1.5 & 2. & & 1. 1 & $9.05 \mathrm{E} \quad 00$ & $3 E \quad 00$ \\
\hline & & & & & 3.17 & 3.8 & $83 E \quad 00$ & $3 \mathrm{E} \quad 01$ \\
\hline & & & $8 E$ & & $1.04 E-03$ & 1.0 & $78 E-01$ & $5 \mathrm{E}-01$ \\
\hline $\mathrm{RA2}$ & 11 & 4. & $9 E-06$ & 5 & $5.61 \mathrm{E}-04$ & 6.7 & $1.56 \mathrm{E}-01$ & $3.87 \mathrm{E}-01$ \\
\hline RA & & & E-03 & & $57 \mathrm{E}-$ & 9.9 & $7.87 \mathrm{E} \quad 00$ & $1.41 E \quad 00$ \\
\hline $\mathrm{C}_{2}$ & 9 & & $3 E$ & & 2 & 3.5 & $5 \mathrm{E} \quad 00$ & $2 \mathrm{E} 01$ \\
\hline & 1 & & $7 E-$ & & 15 & 1.48 & $3 E-03$ & $75 E-03$ \\
\hline & & & 6 & & 3 & 9.5 & $1 E-01$ & $3.87 E-01$ \\
\hline & & & 5 & 1 & 2 & 3.0 & $7.18 \mathrm{E} \quad 00$ & $1.78 \mathrm{E} \quad 01$ \\
\hline & & & $E-02$ & & $8 E-01$ & $1.28 E 00$ & $7.81 \mathrm{E} \quad 00$ & $1.41 \mathrm{E} 00$ \\
\hline & & & $P=-05$ & & $8 \mathrm{E}-04$ & 2. $23 \mathrm{E}-03$ & $48 E-03$ & $E-03$ \\
\hline & 8 & 1. & $2 E-05$ & 4 & $1 E-04$ & 8.9 & $48-01$ & $8 E-01$ \\
\hline & & 1. & $3.83 \mathrm{E}-01$ & -01 & $2 \mathrm{E} \quad 00$ & $1: 03 \mathrm{E} 00$ & $1.00 \mathrm{E} \quad 00$ & $478-01$ \\
\hline & & & ? & & $2.24 E-01$ & $8.73 \mathrm{E}-01$ & $7.57 \mathrm{E} \quad 00$ & $1.71 \mathrm{E} 01$ \\
\hline & & & $6 E$ & & E 01 & 1.56501 & $1.21 \mathrm{E} \quad 01$ & $9.74 \mathrm{E}-01$ \\
\hline & -05 & -04 & $E-03$ & -03 & $E-02$ & $7.84 \mathrm{E}-02$ & $2.98 \mathrm{E}-01$ & $3.17 \mathrm{E}-01$ \\
\hline & 10 & 4.2 & 1. $17 E-01$ & -01 & $9 E-01$ & $2.39 \mathrm{E} 00$ & 3.72500 & $3.62 \mathrm{E} 00$ \\
\hline & -02 & 3. & $8.33 \mathrm{E} \quad 00$ & E 01 & $2 E 01$ & $2.24 E 01$ & $2.17 E 01$ & $1.62 \mathrm{E} 01$ \\
\hline & 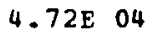 & & $4.56 \mathrm{E} \quad 03$ & & $17 E-06$ & & & \\
\hline & $3 E 04$ & 1.03804 & $1.02 \mathrm{E} \quad 04$ & UE 04 & $9.48 \mathrm{E} \quad 03$ & $7.77 \mathrm{E} \quad 03$ & E 02 & $4.76 E-09$ \\
\hline & $1.51 E \quad 04$ & $1.50 \mathrm{E} \quad 04$ & $1.47 \mathrm{E} 04$ & $16 \mathrm{EO} 04$ & $11 \mathrm{E} 0$ & 142800 & $32 E-01$ & \\
\hline & $2.83 \mathrm{E} \quad 03$ & $2.45 \mathrm{E} 01$ & $1.84 E-03$ & $0 E-18$ & & & & \\
\hline & $4.23 \mathrm{E} 01$ & $4.23 E \quad 01$ & $4.23 E \quad 01$ & $22 E \quad 01$ & $4.21 \mathrm{E} \quad 01$ & 4.15 & $3.52 E \quad 01$ & $6.79 \mathrm{E} \quad 00$ \\
\hline & $4.88 E \quad 04$ & $1.09 \mathrm{E} \quad 05$ & $7.93 \mathrm{E} \quad 04$ & 59F. 04 & $1.05 \mathrm{E} \cap 3$ & $1=43 \mathrm{E}-02$ & & \\
\hline COTA. & $1.24 E 05$ & $1.56 \mathrm{E} \quad 05$ & $1.09 \mathrm{E} 05$ & $96 E \quad 04$ & $2.17 \mathrm{E} \quad 04$ & $1.33 \mathrm{EO} 04$ & $7.96 \mathrm{E} \quad 02$ & $1.77 \mathrm{E}$ \\
\hline
\end{tabular}


Table 4.48. Ingestion toxicity $\left(\mathrm{m}^{3}\right.$ water) of accumulated heavy isotopes in low-level plutonium TRU wastes at a federal repository in the year 2005 as a function of age

\begin{tabular}{|c|c|c|c|c|c|c|c|c|c|c|c|c|}
\hline & $\mathrm{ar}$ & & & & 5 & & & & & & & \\
\hline & 2005 & $\overline{10} \overline{0}$ & 300 & 1000 & 3000 & & 10,00 & 0 & 100 . & $\overline{0}$ & 1.000 .0 & \\
\hline 207 & -06 & -03 & $3.40 \mathrm{E}-02$ & $\mathbf{E}-0$ & $3.72 \mathrm{E}$ & 00 & $3.67 \mathrm{E}$ & 01 & $9.97 \mathrm{E}$ & 02 & $1.42 \mathrm{E}$ & \\
\hline & & & 4.00E- & E- & $1.88 \mathrm{E}$ & 00 & & 01 & & & & \\
\hline & & & & E 01 & & 02 & & & & & & \\
\hline PB2 & 00 & & & 1206 & $92 \mathrm{E}$ & 07 & $1 \mathrm{E}$ & 08 & $8 \mathrm{E}$ & 09 & $9 \mathrm{E}$ & \\
\hline & $4 E-04$ & $E-02$ & $6.82 \cdot \mathrm{E}-0$ & E 00 & $46 \mathrm{E}$ & 0 & & 02 & & 04 & & \\
\hline & & 800 & $9.47 \mathrm{E} 01$ & E 03 & & 04 & $2 E$ & 04 & & 05 & $8 \mathrm{E}$ & 04 \\
\hline$\pi$ & & 01 & $9.30 \mathrm{E} \quad 02$ & 3E 04 & $1.48 \mathrm{E}$ & 05 & & 05 & & 06 & $5 E$ & 06 \\
\hline & & -03 & 3. $90 \mathrm{E}-02$ & $E-01$ & $4.26 \mathrm{E}$ & 00 & & & & 03 & & \\
\hline 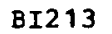 & .05 & $;-01$ & $3.63 \mathrm{E} 00$ & E 02 & $1 \mathrm{E}$ & 03 & $4 \mathrm{E}$ & 04 & $5 \mathrm{E}$ & 05 & & 06 \\
\hline & & 00 & $\begin{array}{ll}9 \mathrm{E} & 01\end{array}$ & E 03 & 9.8 & 03 & & 04 & & 05 & & 04 \\
\hline Po. & & 3 & E 04 & E 06 & & 0 & & & & & & \\
\hline $\mathbf{P}$ & & -05 & $E-03$ & $E-02$ & & -01 & & 00 & & 2 & & 02 \\
\hline & & -03 & $3 E-02$ & $8-01$ & & 00 & & 01 & & 02 & & 01 \\
\hline & & & $E-04$ & $8-03$ & & -0 & & & & 0 & & 01 \\
\hline & & & $1.18 \mathrm{E}$ & 1 & & 0 & & 0 & & 04 & & 04 \\
\hline & 08 & 1. & $3 E-03$ & $8-02$ & & 0 & & 0 & & 02 & E & \\
\hline BN & & & $3 E-0$ & $8-03$ & & -0 & & & & 00 & & \\
\hline $\mathbf{R}$ & & -03 & 4. & & & 0 & & 01 & & 12 & & 01 \\
\hline & & 8. & 2. & B 01 & & 0 & & 0 & & & & \\
\hline & & & $3.90 \mathrm{E} \quad 02$ & $7 \mathrm{E} 03$ & & 0 & $O E$ & 05 & & 07 & & 07 \\
\hline & & $E$ & $3 \mathrm{E} \quad 03$ & E 05 & & 0 & & 0 & & 08 & & 09 \\
\hline & 02 & E 05 & $8 \mathrm{E} \quad 06$ & E 07 & & 0 & & 0 & & 09 & & \\
\hline & -03 & E 0 & $\begin{array}{ll}3 \mathrm{E} & 02\end{array}$ & OE 04 & & 0 & & 0 & & 07 & & 08 \\
\hline & & 1. & $5 \mathrm{E} \quad 02$ & $4 \mathrm{E} \quad 03$ & & 0 & & 0 & & 06 & & 06 \\
\hline & & 0 & E 01 & $1 \mathrm{E} 02$ & & 0 & & 0 & & 0 & & 05 \\
\hline & & 1 & E 03 & E 05 & & 0 & & 0 & & & & \\
\hline & 03 & E OL & E 05 & E 06 & & 0 & & 0 & & 0 & & \\
\hline & 01 & E 02 & $2 \mathrm{E} \quad 02$ & E 03 & & 0 & & 0 & & 04 & $1 \mathrm{E}$ & 04 \\
\hline & $8-01$ & $\mathbf{E}$ & E 02 & E 03 & & 0 & & 0 & & 06 & & 0 \\
\hline & 04 & 1. & E 06 & E 06 & & 0 & & & & 0 & & 0 \\
\hline & $1 \mathrm{E} \quad 00$ & E 02 & 4E 03 & $7 \mathrm{E} 04$ & & 05 & & 06 & & 06 & & 0 \\
\hline & c 08 & E 07 & $00 \quad 07$ & 07 & & .07 & & n7 & & 07 & & \\
\hline & E. 02 & 03 & $\begin{array}{lll}5 E & 03\end{array}$ & E 04 & & 04 & 9. & 04 & & 0 & & \\
\hline & $11 \mathrm{E} \quad 03$ & $5.18 \mathrm{E} \quad 04$ & $1.44 \mathrm{E} 05$ & 1805 & & 06 & $\mathbf{E}$ & 06 & & 06 & & 0 \\
\hline & $7 E \quad 06$ & $0 \mathrm{E} \quad 07$ & $9.45 \mathrm{E} \quad 07$ & $2 E 08$ & & 08 & & & & 08 & & \\
\hline & $85 E \quad 11$ & E 11 & $6 \mathrm{E} \quad 10$ & $8 \mathrm{E} \quad 08$ & & 01 & & & & & & \\
\hline & $4 E \quad 10$ & $6.63 \mathrm{E} \quad 10$ & $6.59 \mathrm{E} \quad 10$ & $16 \mathrm{E} 10$ & & 10 & 5.0 & 1 & & 09 & $3.06 \mathrm{R}$ & \\
\hline & $1 \mathrm{E} 10$ & $9.61 \mathrm{E} \quad 10$ & $9.41 \mathrm{E} \quad 10$ & E 10 & & 1 & & & & & & \\
\hline & $3.41 \mathrm{E} 11$ & 6E 09 & $2.22 \mathrm{E} \quad 05$ & $20 E-10$ & & & & & & & & \\
\hline & $6 \mathrm{E} \quad 08$ & E 08 & $16 \mathrm{E} \quad 08$ & $16 \mathrm{E} \quad 08$ & & 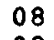 & $2.81 \mathrm{E}$ & & . 398 & 08 & $\mathrm{OE}$ & \\
\hline & & & IE & 4E 11 & & 0 & $7 E$ & 0 & & & & \\
\hline & & & $7.82 \mathrm{E} \quad 11$ & $3.47 \mathrm{E} \quad 11$ & $11 E$ & 11 & $8.70 \mathrm{E}$ & 10 & $1.81 \mathrm{E}$ & 10 & $5.32 \mathrm{E}$ & \\
\hline
\end{tabular}


Table 4.4c. Grams of accumulated heavy elements in low-level J-233 wastes at a federal repository in the year 2005 as a function of age

\begin{tabular}{|c|c|c|c|c|c|c|c|c|}
\hline & $\begin{array}{l}\text { Year } \\
2005\end{array}$ & 100 & 300 & $\frac{1}{10} \frac{1}{10} \frac{\mathrm{g}}{\mathrm{w}} \mathrm{w}$ & cl $\frac{1}{30}$ 오을 & $\frac{h}{10}$ & 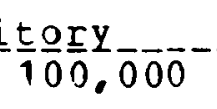 & 000 \\
\hline E. & $8.95 \mathrm{E}-02$ & . & 00 & 00 & 5.7 & 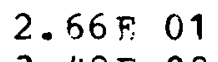 & 2 & 03 \\
\hline 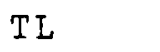 & & -0 & -08 & $E-10$ & $F-09$ & -0 & $-0 a$ & -10 \\
\hline$B$ & 01 & 00 & E 00 & E 00 & $E \quad 00$ & $E 0$ & 02 & 03 \\
\hline- & 04 & -0 & $8-01$ & E 00 & 01 & 0 & 3 & \\
\hline 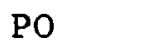 & & c. & -06 & $E-05$ & -05 & -04 & 3 & -04 \\
\hline $\mathrm{T}$ & -14 & -1 & $5 E-12$ & $E-12$ & $F-11$ & & 1 & -13 \\
\hline . & .07 & $E-0$ & $3 E-08$ & $E-07$ & $e-06$ & 5 & 1. & 05 \\
\hline FR & -10 & $E-0$ & $9 E-08$ & $E-08$ & $8-07$ & & 07 & -09 \\
\hline & 4 & $E-C$ & $5 E-03$ & $E-02$ & $:-01$ & 00 & 01 & 00 \\
\hline$A C$ & -06 & $E-05$ & $8 E-05$ & $E-04$ & -04 & -0.3 & $E-03$ & $E-0.5$ \\
\hline $\mathrm{TH}$ & -01 & 00 & 2501 & 01 & 02 & 02 & 03 & 02 \\
\hline PA & $=-05$ & $E-04$ & $y-04$ & $z-03$ & $E-03$ & $E-02$ & $\mathrm{E}-02$ & $E-02$ \\
\hline $\mathbf{U}$ & 04 & E 04 & $2 \mathrm{E} \quad 04$ & E 04 & $\begin{array}{ll}E & 04\end{array}$ & E 04 & E 04 & \pm 03 \\
\hline 0 & $2 \mathrm{E} 04$ & $1.92 \mathrm{E} \quad 04$ & $2 \mathrm{E} \quad 04$ & $2 \mathrm{E} \quad 04$ & $2 \mathrm{E} \quad 04$ & $92 E \quad 04$ & $1.92 E 04$ & $1.92 \mathrm{E} \quad 04$ \\
\hline
\end{tabular}


Table 4.50. Curies of accumulated heavy isotopes in low-level U-233 wastes at a federal repository in the year 2005 as a function of age

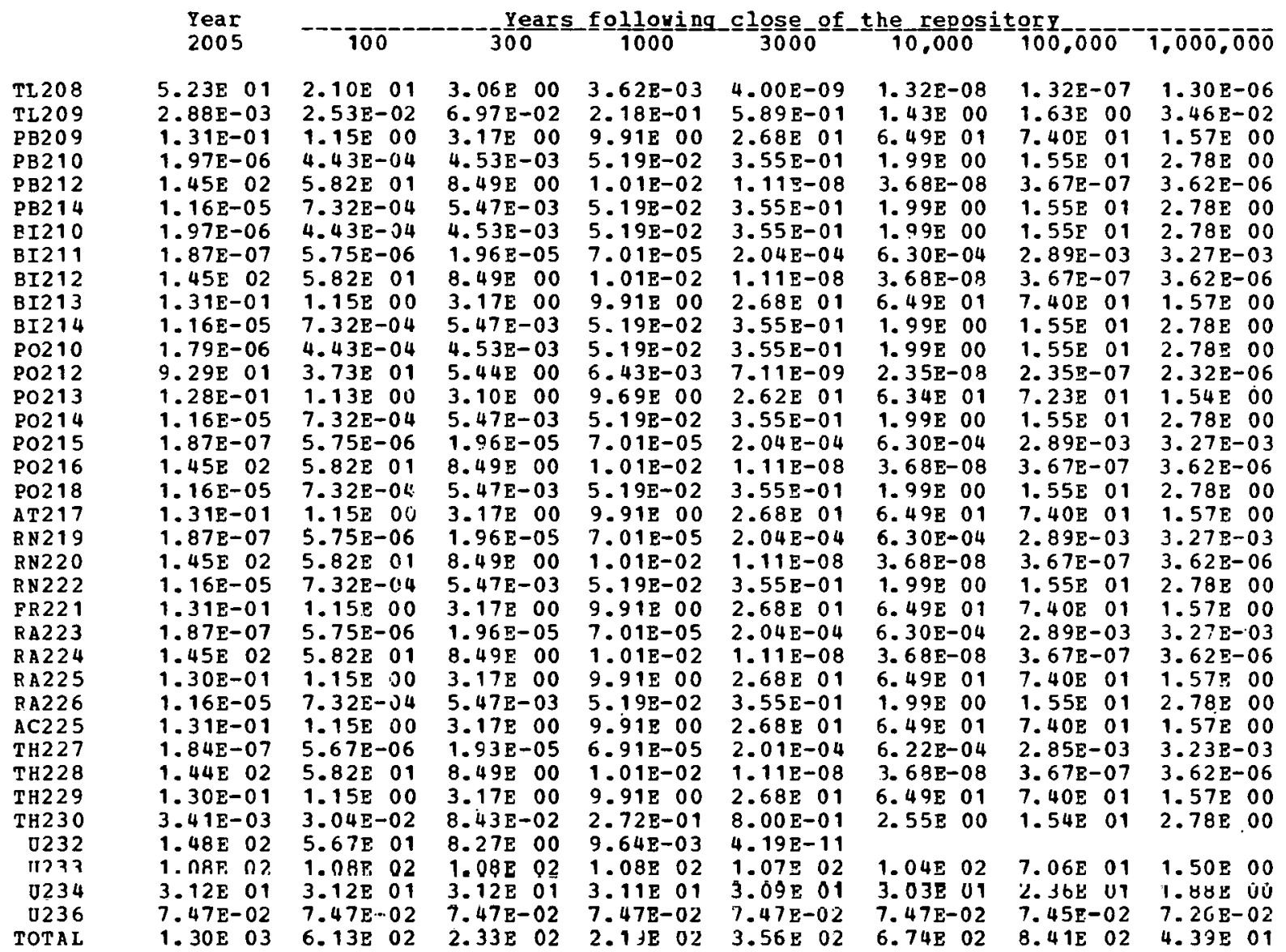


Table 4.51. Watts of accumulated heavy isotopes in low-level U-233 wastes at a federal repository in the year 2005 as a function of age

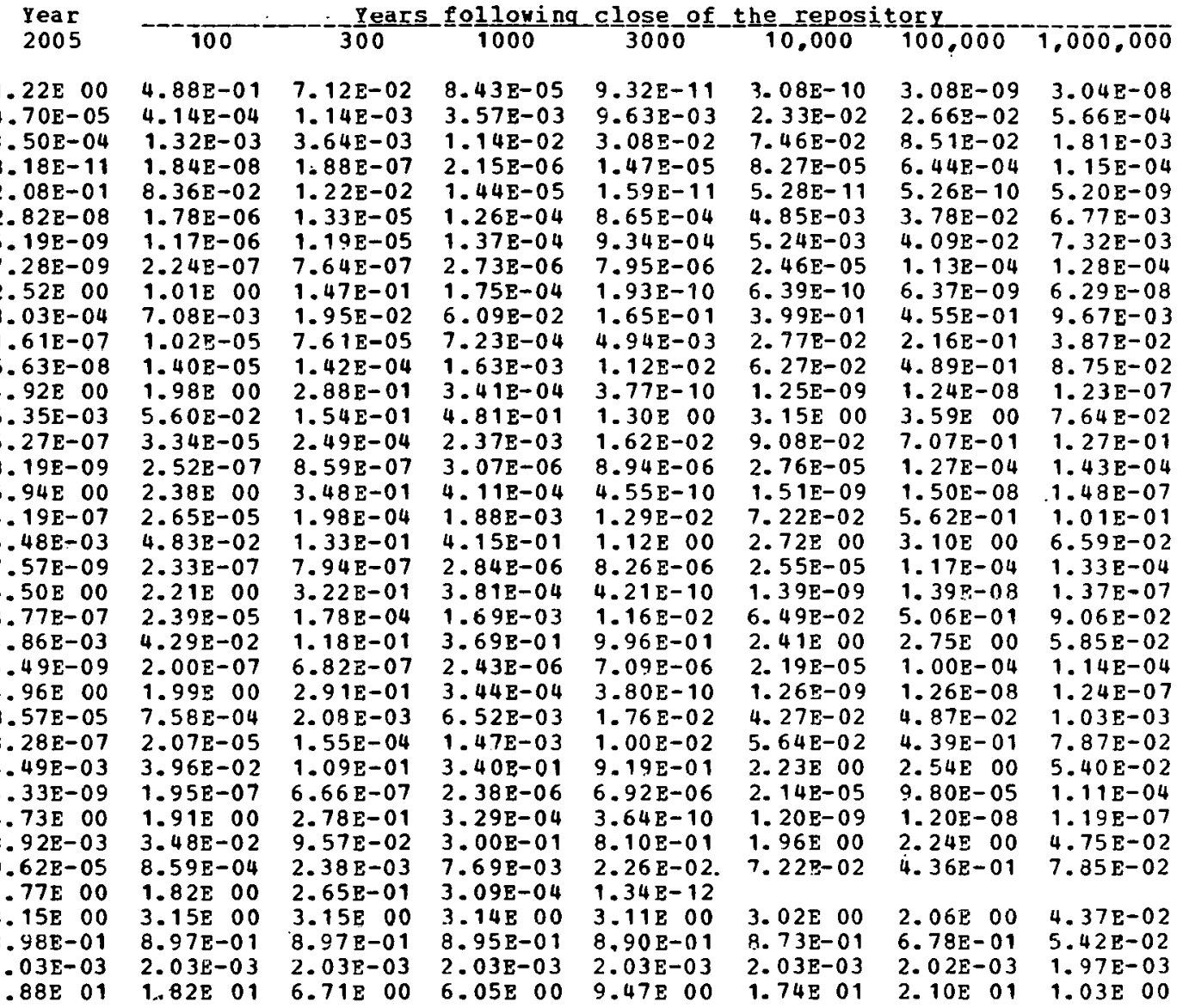


Table 4.52. Ingestion toxicity $\left(\mathrm{m}^{3}\right.$ water) of accumulated heavy isotopes in low-level U-233 wastes at a federal repository in the year 2005 as a function of age

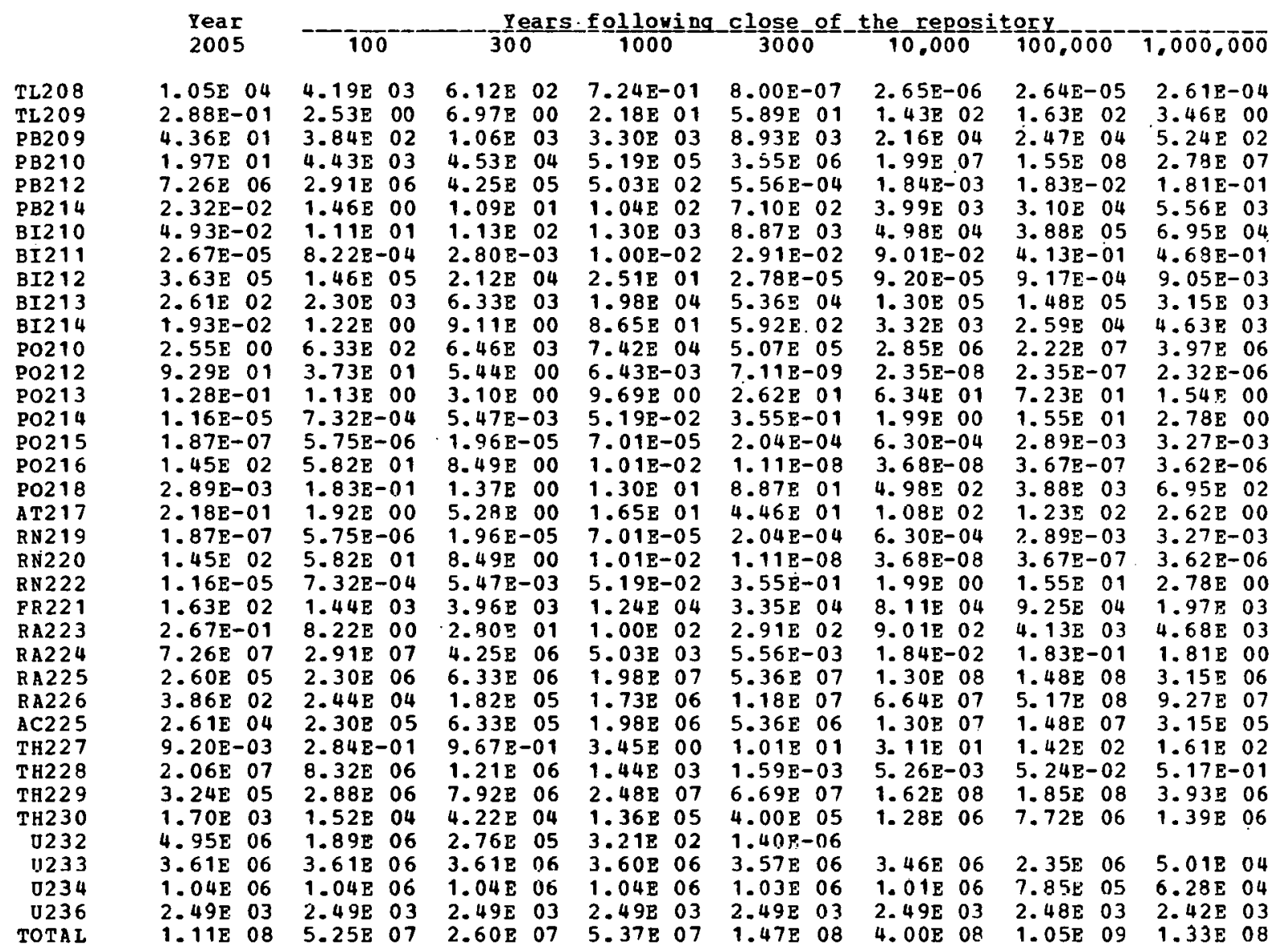


Table 4.53. Projected annual accumulation of noble-gas fission products

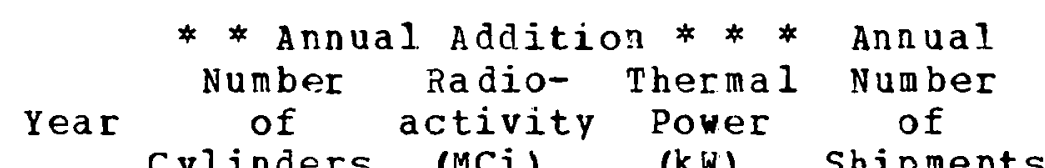

Cylinders (MCi) (KW) Shipments

$\begin{array}{lr}1980 & 0 \\ 1981 & 0 \\ 1982 & 21 \\ 1983 & 62 \\ 1984 & 69 \\ 1985 & 78 \\ 1986 & 111 \\ 1987 & 164 \\ 1988 & 160 \\ 1989 & 187 \\ 1990 & 238 \\ 1991 & 264 \\ 1992 & 321 \\ 1993 & 352 \\ 1994 & 411 \\ 1995 & 442 \\ 1996 & 504 \\ 1997 & 532 \\ 1938 & 613 \\ 1999 & 678 \\ 2000 & 621 \\ 2001 & 582\end{array}$

$0.0 \quad 0.1 \quad 0$

0.1

0.1

2. 8

4. 2

8.3

12.5

9.2

13.9

14.7

15.6

22.1

32.4

31.3

20.8

23.8

35.8

45.7

51.0

61.8

68.3

70.4

84.2

0.5 .0

99.9

116.4

130.3

$11 \varepsilon .9$

110.6

73.4

TIME AFTER SHUTDOWN, YEARS

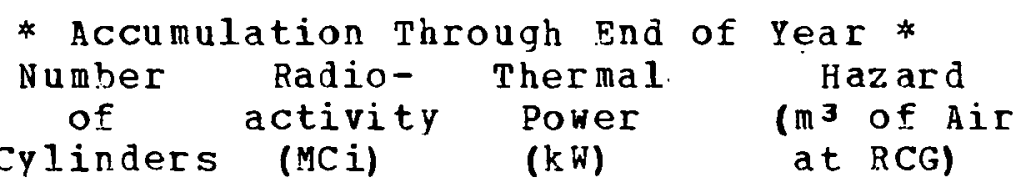
$\begin{array}{lll}\text { of } & \text { activity } & \text { Power } \\ \text { linders } & \text { (MCi) } & \text { (kW) }\end{array}$ $\begin{array}{lll}\text { of activity } & \text { Power } \\ \text { linders } & \text { (MCi) } & (\mathrm{kW})\end{array}$

$\mathrm{Haz}$ ard

(m of $m^{3}$ ir
at RCG)

0
0
21
84
153
231
342
505
666
853
1090
1355
1675
2027
2438
2880
3384
3916
4529
5207
5829
6410

0
0
3
11
20
29
42
61
78
97
121
148
180
214
253
294
339
384
438
497
546
585

0
0
4
17
29
43
63
91
117
145
182
222
270
322
381
442
509
578
658
748
820
830

$1.4 \mathrm{E} \quad 11$ 3. $8 \mathrm{E} \quad 11$ 9.7 F 12 $3.7 \mathrm{E} \quad 13$ $6.5 \mathrm{E} 13$ $9.6 \mathrm{E} 13$ $1.4 E 14$ 2. $0 F, 14$ $2.6 \mathrm{E} \quad 14$ 3. $2 \mathrm{E} 14$ 4. $0 \mathrm{E} 14$ $4.9 \mathrm{E} 14$ $6.0 \mathrm{E} 14$ 7. $1 \mathrm{E} \quad 14$ $8.4 \mathrm{~F} 14$ 9. $8 \mathrm{E} 14$ 1. 1 E 15 1. $3 \mathrm{E} 15$ 1. $5 \mathrm{~F} 15$ 1. $7 \mathrm{E} \quad 15$ $1.8 \mathrm{E} \quad 15$ 2. 0 . 15 
and then shipped by rail to a repository for long-term storage. An individual shipment consists of six gas cylinders contained in a specially-designed water-filled cask. ${ }^{30}$

\subsection{Iodine}

Iodine is a semivolatile fission product which, because of its complex physical and chemical properties and its high biological significance, has always required special attention to ensure adequate safety in its management. About $0.01 \mathrm{~g}$ of iodine isotopes is formed per MWd (thermal), and the isotope of greatest concern during reactor operation and fuel reprocessing is 8.05-d ${ }^{131} \mathrm{I}$. However, the species of consequence to longer-term waste management is $1.6 \times 10^{7}-y^{129} \mathrm{I}$, which comprises about $75 \%$ of the weight of the fission product iodine isotopes in spent fuels at the time of reprocessing.

Research and development work aimed at reducing iodine releases from fuel reprocessing plants to "near zero" levels shows promise of removing at least $99.9 \%$ of it from other fuel constituents by volatilization at the head-end of the process. ${ }^{29}$ Once separated, it would subsequently be trapped and retained separately from the other waste streams. The final form into which the iodine may be processed for packaging, shipment, and disposal has not been defined as yet, but concentrates of ${ }^{129}$ I must receive careful attention bccause of this isotope's very long half-life.

The accumulation of fission product iodine is given in Table 4.54 . In constructing this table, it was assumed that $99.9 \%$ of the iodine was recovered in a pure form at the reprocessing plants, converted to barium iodate, concreted, and stored for 1 year before shipment to a repository or to a disposal site. The barium iodate was assumed to be concreted with a mixture of cement, water, and $0.9 \mathrm{wt} \%$ butyl stearate (to reduce the iodine leach rate), resulting in a product containing $10 \mathrm{wt} \%$ iodine. ${ }^{31}$ On a volumetric basis, the product contains $5.29 \mathrm{~kg}$ of iodine per cubic foot. It is shipped by motor freight in Type 17C, 55-gal drums, 64 drums per shipment. The minimum number of annual shipments is taken to be one per reprocessing plant. 
Table 4.54. Projected annual accumulation of fission product iodine

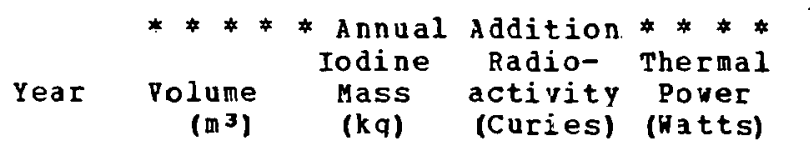

$\begin{array}{ll}1980 & 0.00 \\ 1981 & 0.01\end{array}$

$\begin{array}{ll}1981 & 0.01 \\ 1982 & 0.44\end{array}$

$1983 \quad 1.30$

$1984 \quad 1.44$

$1985 \quad 1.62$

$1986 \quad 2.30$

1987

1988

1989
1990

1991

1992

1993

1994
1995

1995
1996

1997

1998

1999

2000

3.45

3.44
4.13

5.20

5.75

7.01

7.60

8.92

11.34

12.03

13.72

14.94

13.92

13.35

1
1
81
243
270
303
429
644
643
772
972
1074
1309
1419
1666
1836
2118
2247
2564
2790
2600
2493

(Curies) (Hatts)

\subsection{0 .000}

0.20 .000

$11.2 \quad 0.007$

$33.5 \quad 0.020$

$\begin{array}{ll}37.1 & 0.022 \\ 41.7 & 0.025\end{array}$

$59.0 \quad 0.03$

$88.5 \quad 0.036$

88.20 .053

$105.6 \quad 0.064$

133.10 .080

$147.2 \quad 0.089$

$179.3 \quad 0.108$

$194.6 \quad 0.118$

228.30 .138

$251.1 \quad 0.152$

$289.4 \quad 0.175$

306.90 .185

$350.4 \quad 0.212$

$381.8 \quad 0.231$

$355.0 \quad 0.215$

0.206

TIME AFTER SHUTDOHN, YEARS
Annual

Shipments

0
0
1
4
5
6
8
12
12
14
18
20
24
26
30
33
39
41
47
51
47
45

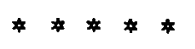

Volu

(in $\left.{ }^{3}\right)$

\subsection{0}

0.01

0.45
1.75

1.75

3.19

4.82

10.56

14.00

18.13

23. 33

29.09

36.09

43.69

52.61

62.43

73.77

85.80 99.52

114.46

128.38

141.73

141.73

141.73

141.73

141.73

141.73
141.73

141.73

141.73

141.73
Accumulation Through End of Year ****** Iodine Radio- Thermal Hazard, Cubic

Mass activity Power Meters at RCG

(kg) (Curies) (Hatts) Air Water

$\begin{array}{rrr}1 & 0 & 0.000 \\ 2 & 0 & 0.000 \\ 83 & 11 & 0.007 \\ 327 & 45 & 0.027 \\ 597 & 82 & 0.050 \\ 900 & 124 & 0.075 \\ 1329 & 183 & 0.111 \\ 1973 & 271 & 0.164 \\ 2615 & 360 & 0.217 \\ 3387 & 465 & 0.281 \\ 4359 & 598 & 0.362 \\ 5434 & 746 & 0.451 \\ 6743 & 925 & 0.559 \\ 8162 & 1120 & 0.677 \\ 9828 & 1348 & 0.815 \\ 11663 & 1599 & 0.966 \\ 13781 & 1888 & 1.141 \\ 16029 & 2195 & 1.327 \\ 18592 & 2546 & 1.538 \\ 21383 & 2927 & 1.769 \\ 23983 & 3282 & 1.984 \\ 26477 & 3622 & 2.190\end{array}$

4. $4 \mathrm{E} 09$ 1.3E 10 4. $2 \mathrm{E} \quad 06$ $5.7 \mathrm{E} 11 \quad 1 . \subseteq \mathrm{E} \quad 08$ 2.2E $12 \quad 7.5 \mathrm{E} \quad 08$ 4.1E $12 \quad 1.4 \mathrm{E} \quad 09$ $6.2 \mathrm{E} 12$ 2.1. 09 $9.1=12 \quad 3.0=09$ 3. $0 \mathrm{E} 09$ $1.4 \mathrm{E} 13-4.5 \mathrm{E} 09$ $1.8 \mathrm{E} 13$ 6. 13 E 09 2.3 E 13 7. $3 \mathrm{E} \quad 09$ $3.0 \mathrm{E} 13 \quad 1.0 \mathrm{E} \quad 10$ $\begin{array}{llll}3.7 \mathrm{E} & 13 & 1.2 \mathrm{E} & 10\end{array}$ $\begin{array}{llll}4.6 \mathrm{E} & 13 & 1.5 \mathrm{E} & 10\end{array}$ $5.6 \mathrm{E} 13 \quad 1.9 \mathrm{E} \quad 10$ $6.7 \mathrm{E} 13 \quad 2.2 \mathrm{E} 10$ $\begin{array}{llll}6.7 \mathrm{E} & 13 & 2.2 \mathrm{E} & 10 \\ 8.0 \mathrm{E} & 13 & 2.7 \mathrm{E} & 10\end{array}$ $\begin{array}{llll}8.0 \mathrm{E} & 13 & 2.7 \mathrm{E} & 10 \\ 9.4 \mathrm{E} & 13 & 3.1 \mathrm{E} & 10\end{array}$ $1.1 E 14 \quad 3.7 E \quad 10$ 1.3E $14 \quad 4.2 E \quad 10$ $1.5 \mathrm{E} 1.4 \quad 4.9 \mathrm{E} 10$ $\begin{array}{llll}1.6 \mathrm{E} & 14 & 5.5 \mathrm{E} & 10\end{array}$ $\begin{array}{llll}1.6 E & 14 & 5.5 E & 10 \\ 1.8 E & 14 & 6.0 E & 10\end{array}$

$\begin{array}{lll}26477 & 3622 & 2.190 \\ 26477 & 3622 & 2.190 \\ 26477 & 3622 & 2.190 \\ 26477 & 3621 & 2.189 \\ 26477 & 3620 & 2.189 \\ 26477 & 3617 & 2.187 \\ 26477 & 3606 & 2.180 \\ 26477 & 3575 & 2.161 \\ 26477 & 3468 & 2.097\end{array}$

$\begin{array}{lllll}1.8 \mathrm{E} & 14 & 6.0 \mathrm{E} & 10\end{array}$ $1.8 \mathrm{E} 14 \quad 6.0 \mathrm{E} 10$ $\begin{array}{llll}1.8 \mathrm{E} & 14 & 6.0 \mathrm{E} & 10\end{array}$ $1.8 \mathrm{E} 14$ 6. $14 \mathrm{E} 10$ $\begin{array}{llll}1.8 \mathrm{E} & 14 & 6.0 \mathrm{E} & 10\end{array}$ $\begin{array}{llll}1.8 \mathrm{E} & 14 & 6.0 \mathrm{E} & 10 \\ 1.8 \mathrm{E} & 14 & 6.0 \mathrm{E} & 10\end{array}$ $1.8 \mathrm{E} 14 \quad 6.0 \mathrm{E} 10$

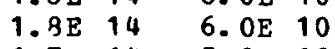
$\begin{array}{llll}1.7 \mathrm{E} & 14 & 5.8 \mathrm{E} & 10\end{array}$ 


\subsection{Carbon-14}

Carbon-14 is produced in oxide-fueled reactors principally by an $(n, p)$ reaction with ${ }^{14} \mathrm{~N}$ impurity in the fuels, but also as the product of an $(n, \alpha)$ reaction with ${ }^{17} O$. In HTGRs, it is also produced by $(n, \alpha)$ reactions with the graphite fuel blocks. The chemical behavior of carbon during nitric acid dissolution of the fuel is not known. The assumption most often made is that it will be released as a gas, most probably $\mathrm{CO}_{2}$, from the dissolver. In HTGR fuel reprocessing, the ${ }^{14} \mathrm{C}$ will be oxidized with the graphite in the fuel block burning step.

The ${ }^{14} \mathrm{C}$ activity which appears in the fuel reprocessing plant depends on the amount of nitrogen impurity in the fuel. A value of $25 \mathrm{ppm}$ nitrogen has been recommended for LWR fuels, although values from 1 to $100 \mathrm{ppm}$ have been observed. ${ }^{32}$ The nitrogen content of HTGR fuel blocks is assumed to be $30 \mathrm{ppm}$, in close agreement with values used elsewhere. ${ }^{33}$ The nitrogen content of LMFBR fuel is also assumed to be $25 \mathrm{ppm}$. The ${ }^{14} \mathrm{C}$ activities resulting in spent fuels from the reference reactors are as follows:

Reactor

PWR-U

$\mathrm{PWR}-\mathrm{Pu}$

BWR-U

$\mathrm{BWR}-\mathrm{Pu}$

HTGR

LMFBR

${ }^{14}$ C Activity
(Ci/MTHM)

The projected annual accumulations of ${ }^{14} \mathrm{C}$ which will be generated by LWRs and LMFBRs are given in Table 4.55.

\subsection{Tritium}

Tritium wastes are generated at nuclear power stations and at fuel reprocessing plants. Tritium in light water reactor wastes arises principally from neutron reactions with light elements such as lithium and boron that may be present in the primary coolants. New power 
Table 4.55. Projected annual accumulation ( $\mathrm{C} i$ ) of carbon-14 from reactor fuels

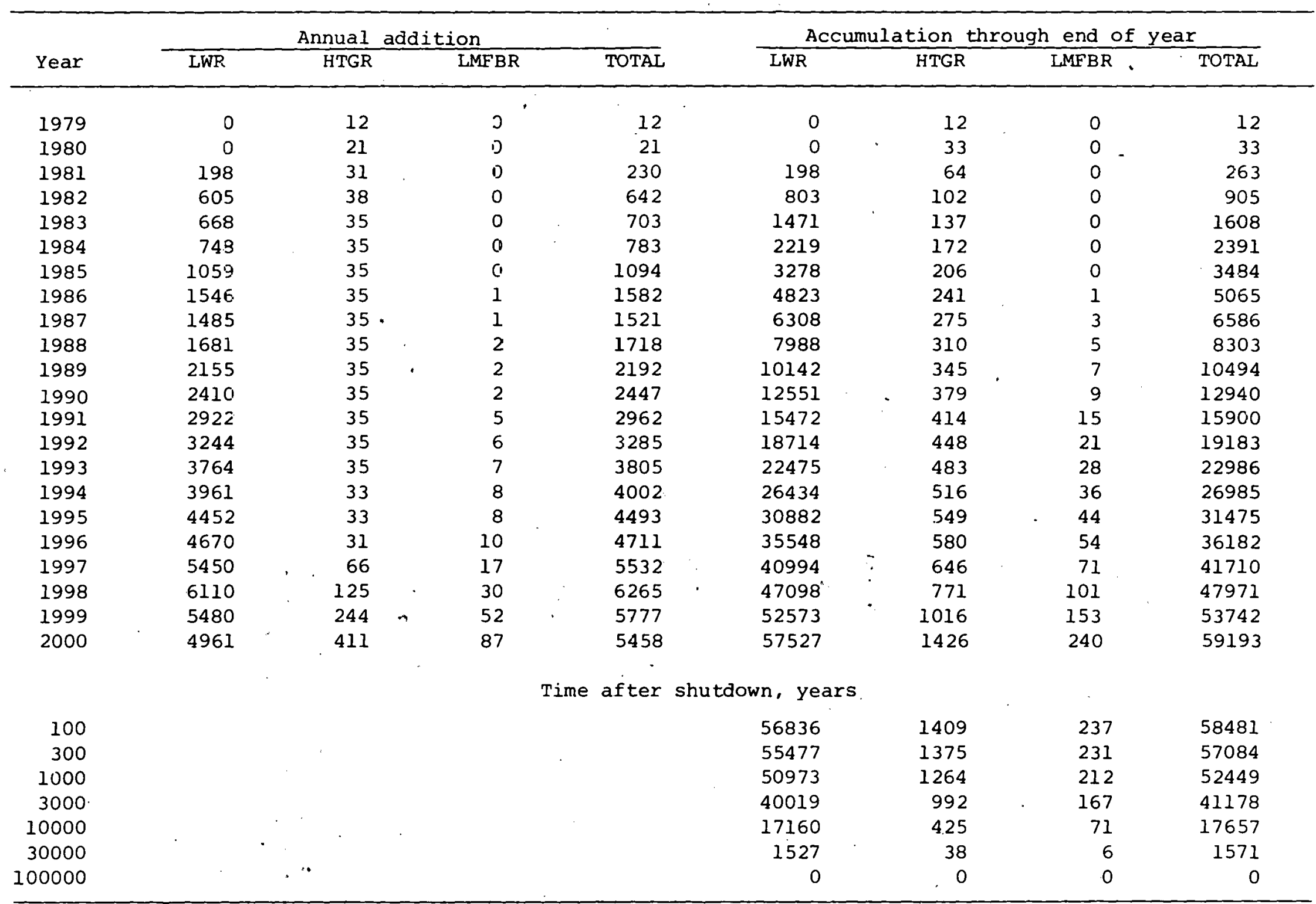


stations may have the capability for recycling the coolant until the tritium concentration reaches about $5 \mu \mathrm{Ci} / \mathrm{ml}$, and then to maintain that concentration by additions of fresh water to the coolant as required. Operating experience with this technique is lacking; however, using the annual rates of tritium generation reported in ref. 34 , it is estimated that about 63,380 and 10,560 gal of tritiated water will be bled annually from the primary circuits of the reference PWR and BWR, respectively. This waste could be stored in tanks at the power stations until it is shipped to disposal sites.

Between 400 and $1300 \mathrm{Ci}$ of tritium per metric ton of fuel, depending on the type of reference reactor, is produced in fission and appears in wastes from fuel reprocessing. A few percent may be associated with the fuel cladding, but most is present with the core materials and is eventually released as water vapor to the atmosphere. Future plants having head-end operations like "voloxidation"17 should be able to separate and recover the tritium in a relatively small volume, perhaps in as little as a few liters of tritiated water per day. This concentrate could then be converted into an appropriately stable, solid form [such as $\mathrm{Ca}(\mathrm{OH})_{2}$, perhaps], packaged, and shipped to a designated storage or disposal site.

There is evidence that tritium may act differently in LMFBRs than in other reactor types. ${ }^{34}$ As much as $95 \%$ may diffuse through the stainless steel cladding during reactor operation and appear as a sodium tritide sludge in the primary-coolant cold traps. If experience bears out this behavior, processes for recovery and packaging of the tritium from LMFBR fuels will be installed at the power stations rather than at the reprocessing plants.

The projected accumulation of tritium in the form of tritiated water from the primary coolant circuits of LWRs is presented in Table 4.56. After storage at the power stations for one year, this water is shipped to a disposal site in tank trucks of 4000-gal capacity each. 
Table 4.56. Projected annual accumulation of tritiated water from reactors

* Annual Addition * * Annual Volume Radio-
Year (Thousands activity
of $m^{3}$ )
(MCi)

$\begin{array}{llll}0.56 & 0.0026 & 0.09 & 37 \\ 0.90 & 0.0042 & 0.15 & 59\end{array}$

$1.040 .0042 \quad 0.15 \quad 59$

$\begin{array}{llll}1.04 & 0.0049 & 0.17 & 69\end{array}$

$\begin{array}{llll}1.60 & 0.0076 & 0.27 & 106\end{array}$

$\begin{array}{llll}3.68 & 0.0174 & 0.62 & 243\end{array}$

$\begin{array}{llll}5.79 & 0.0274 & 0.97 & 382\end{array}$

$\begin{array}{llll}6.66 & 0.0315 & 1.12 & 440\end{array}$

$\begin{array}{llll}7.85 & 0.0371 & 1.32 & 518\end{array}$

$\begin{array}{llll}8.57 & 0.0405 & 1.44 & 566\end{array}$

$\begin{array}{llll}9.48 & 0.0448 & 1.59 & 626\end{array}$

$\begin{array}{llll}10.78 & 0.0509 & 1.81 & 712\end{array}$

$\begin{array}{llll}12.70 & 0.0500 & 2.14 & 839\end{array}$

$\begin{array}{llll}15.00 & 0.0709 & 2.52 & 991\end{array}$

$17.91 \quad 0.0846 \quad 3.01 \quad 1183$

$\begin{array}{llll}20.22 & 0.0955 & 3.40 & 1335\end{array}$

$\begin{array}{llll}22.98 & 0.1086 & 3.87 & 1518\end{array}$

$25.75 \quad 0.1217$

28.66

31.71

34.64

37.83

41. 01

44.34

47.66

50.86

53.90

56.72

59.49

62.26

64.83

0.1217

0.1499

0.1637

0.1787
0.1938

0.2095

0.2252

0.2403

0.2547

0.2680

0.2811

0.2942$$
4.33
$$

1701

$4.82 \quad 1893$

5.332095

$5.83 \quad 2288$

$6.36 \quad 2498$

$6.90 \quad 2709$

7.462928

8.023148

$9.07 \quad 3560$

$9.54 \quad 3747$

$10.01 \quad 3929$

$\begin{array}{ll}10.01 & 3929 \\ 10.47 & 4112\end{array}$

TIME AFTER SHUTDORy, TEARS

100

300

1000

3000

10000

* * Accumulation Through End of Year * * * * Volume Radio- Thermal Hazard, Cubic

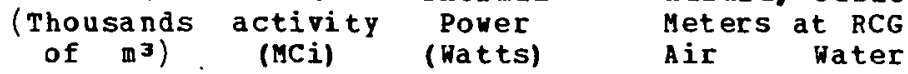

$\begin{array}{rll}0.6 & 0.003 & 0.1 \\ 1.5 & 0.007 & 0.2 \\ 2.5 & 0.011 & 0.4 \\ 4.1 & 0.018 & 0.6 \\ 6.4 & 0.028 & 1.0 \\ 10.1 & 0.044 & 1.6 \\ 15.9 & 0.069 & 2.5 \\ 22.6 & 0.097 & 3.4 \\ 30.4 & 0.128 & 4.6 \\ 39.0 & 0.162 & 5.8\end{array}$

$\begin{array}{lllll}1.3 \mathrm{E} & 10 & 8.8 \mathrm{E} & 05\end{array}$

$3.4 \mathrm{E} 10 \quad 2.2 \mathrm{E} 06$

$5.6 \mathrm{E} 10 \quad 3.8 \mathrm{E} 06$

$9.1 \mathrm{E} 10 \quad 6.1 \mathrm{E} 06$

$1.4 \mathrm{E} 11 \quad 9.4 \mathrm{E} 06$

2. 2 E $11 \quad 1.5 \mathrm{E} \quad 07$

$\begin{array}{llll}3.5 \mathrm{E} & 11 & 2.3 \mathrm{E} & 07\end{array}$

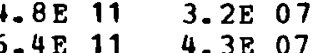

$8.1 \mathrm{E} 11 \quad 5.4 \mathrm{E} 07$

$9.9 E 11$ 6.6 07

$1.2 \mathrm{E} 12 \quad 7.3 \mathrm{E} 07$

$1.2 \mathrm{E} 12$ 7. $12 \mathrm{E} 07$

$1.4 \mathrm{E} 12 \quad 9.5 \mathrm{E} 07$

$\begin{array}{llll}1.7 \mathrm{E} & 12 & 1.1 \mathrm{E} & 08\end{array}$

$2.0 \mathrm{E} 12 \quad 1.4 \mathrm{E} \quad 8$

$2.4 \mathrm{E} 12 \quad 1.6 \mathrm{E} 08$

$\begin{array}{lllll}2.8 \mathrm{E} & 12 & 1.9 \mathrm{E} & 08\end{array}$

$3.3 \mathrm{E} 12 \quad 2.2 \mathrm{E} 08$

$3.8 \mathrm{E} 12 \quad 2.5 \mathrm{E} 08$

$4.3 \mathrm{E} 12$ 2.

$\begin{array}{llll}4.9 \mathrm{E} & 12 & 3.3 \mathrm{E} & 08\end{array}$

$5.5 \mathrm{E} 12$ 3.7 E 08

$6.2 \mathrm{E} 12$ 4. $12 \mathrm{E} \quad 08$

$\begin{array}{lllll}6.9 \mathrm{E} & 12 & 4.6 \mathrm{E} & 08\end{array}$

$\begin{array}{lllll}7.6 \mathrm{E} & 12 & 5.1 \mathrm{E} & 08\end{array}$

$8.4 \mathrm{E} 12 \quad 5.6 \mathrm{E} \quad 08$

$9.2 \mathrm{E} 12 \quad 6.1 \mathrm{E} 08$

$1.0 \mathrm{E} 13 \quad 6.7 \mathrm{E} 08$

1.18 13 7.3 E 08

1.2E 13 7. SE 08

$8.4 \mathrm{E} 08$

$\begin{array}{lllll}787.7 & 0.009 & 0.3 & 4.4 \mathrm{E} 10 & 3.0 \mathrm{E} 06 \\ 787.7 & 0.000 & 0.0 & 5.5 \mathrm{E} 05 & 3.6 \mathrm{E} 01 \\ 787.7 & 0.000 & 0.0 & 3.5 \mathrm{E}-12 & 2.4 \mathrm{E}-16 \\ 787.7 & 0.000 & 0.0 & 2.8 \mathrm{E}-61 & 1.8 \mathrm{E}-65 \\ 787.7 & 0.0 & 0.0 & 0.0 & 0.0\end{array}$


Shipments and accumulations of fission-product tritium one year after its separation from spent fuel at reprocessing plants and LMFBR power stations are presented in Table 4.57. It: is assumed that this tritium is recovered in water at a concentration of $200 \mathrm{Ci} / \mathrm{liter}$ and is then immobilized as $\mathrm{Ca}(\mathrm{OH})_{2}$. The tritiated $\mathrm{Ca}(\mathrm{OH})_{2}$ is packaged for shipment in Foamglas shipping containers $\left[0.16 \mathrm{ft}^{3}\right.$ of $\mathrm{Ca}(\mathrm{OH})_{2}$ per container] and shipped by motor freight to a repository for long-term storage. Each shipment consists of 64 containers carrying a total net payload of $10.2 \mathrm{ft}^{3}$ of $\mathrm{Ca}(\mathrm{OH})_{2}$.

\subsection{Low-Level Non-TRU Solid Wastes}

These diverse solid wastes are common to all facilities handling radioactive materials. They range from concentrates of the radionuclides generated in the decontamination of plant effluent streams to almost every conceivable type of contaminated solid refuse from plant operations. They normaliy require minimal shielding; and, since they contain less than $10 \mathrm{nCi}$ of long-lived alpha activity per gram, they are currently disposable in surface burial grounds. Based on operating experiences at ERDA sites, we estimate that 40,000, 80,000, and 16,000 $\mathrm{ft}^{3}$ of these wastes per ton of plutonium or ${ }^{23} \mathrm{U}$ processed will be generated at fuel preparation, fabrication, and reprocessing plants, respectively. Their average radioactivity level is taken to be $1 \mathrm{Ci}$ of a 160-day-old LWR fission-product mixture per cubic foot. Additionally, we estimate that 15,000 $\mathrm{ft}^{3} /$ year will be generated at each 1000-MW(e) power station. ${ }^{35}$ These reactor wastes are assumed to contain 0.1 , 0.2 , and $0.3 \mathrm{Ci}$ of a 160-day-old mixture of induced (corrosion product) isotopes per cubic foot during the years 1971 to 1985,1986 to 1990 , and 1991 to 2000, respectively. The wastes are packaged in Type 17C, 55-gal steel drums and shipped by motor freight to licensed burial grounds 1 year after their generation. Individual shipments consist of 64 drums containing a total of $475 \mathrm{ft}^{3}$ of waste, and 1 acre of land is utilized for the burial of each $50,000 \mathrm{ft}^{3}$ of waste.

The projected annual and accumulated amounts of compacted, solid non-TRU wastes from reactors at one or more surface burial grounds 
Table 4.57. Projected annual accumulation of fission product tritium

* Annual Addition * $*$ * Annual
Year Volume activity Power
(m) (MCi) (Hatts) Shipments

\section{0}

1981

1982

1983

1984

1985

1986

1987

1988

1989

1990

1991

1992

1993

1994

1995

1996

1997

1998

1999

2000

2001

TIME AFTER SHUTDOWN, YEARS

$\begin{array}{rrrr}0.0 & 0.00 & 0 & 0 \\ 0.0 & 0.00 & 0 & 0 \\ 3.1 & 0.16 & 6 & 11 \\ 9.3 & 0.48 & 17 & 32 \\ 10.3 & 0.54 & 19 & 36 \\ 11.6 & 0.61 & 22 & 40 \\ 16.5 & 0.86 & 31 & 57 \\ 24.5 & 1.28 & 46 & 95 \\ 24.7 & 1.27 & 45 & 85 \\ 29.1 & 1.52 & 54 & 101 \\ 36.7 & 1.91 & 68 & 127 \\ 40.7 & 2.12 & 75 & 141 \\ 49.5 & 2.58 & 92 & 171 \\ 53.9 & 2.81 & 100 & 187 \\ 63.2 & 3.29 & 117 & 219 \\ 69.1 & 3.60 & 128 & 239 \\ 79.4 & 4.14 & 147 & 275 \\ 84.1 & 4.39 & 156 & 291 \\ 96.4 & 5.02 & 173 & 334 \\ 105.6 & 5.51 & 195 & 366 \\ 98.2 & 5.12 & 182 & 340 \\ 94.0 & 4.90 & 174 & 325\end{array}$

\begin{tabular}{|c|c|c|c|}
\hline $\begin{array}{c}\text { Volume } \\
\left(\mathrm{m}^{3}\right)\end{array}$ & $\begin{array}{c}\text { Accumulation } \\
\text { Radio- } \\
\text { activity } \\
\text { (MCi) }\end{array}$ & $\begin{array}{l}\text { Through } \\
\text { Thermal } \\
\text { Power } \\
\text { (Hatts) }\end{array}$ & $\begin{array}{l}\text { of Year } * \text { * } \\
\text { Hazard, Cubic } \\
\text { Meters at RCG } \\
\text { Air Hater }\end{array}$ \\
\hline
\end{tabular}

\section{$0.0 \quad 0.00$}

$0.0 \quad 0.00$

3. 10.16

12.4

2.2. 8

34.4

50.8

75.5

99.9

129.0

165.7

206.3

255.9

309.8

373.0

442.0

521.4

605.5

701.9

807.5

905.6

999.6

0.64

1.14
1.69

2. 45

3.60

4. 68

5.94

7.52

9.23

11.31

13.50

16.05

18.77

21.88

25.06

28.71

32.63

35.96

38.88

0
0
6
23
41
60
87
128
166
211
268
328
402
480
571
667
778
891
1021
1160
1279
1383

4. 2 E 09

1. 2 E 10

8. $2 \mathrm{E} 11$

3. $2 \mathrm{E} 12$

$5.7 \mathrm{E} \quad 12$

8. 4 E 12

1. $2 \mathrm{E} 13$

1. $8 \mathrm{E} 13$

2. 3 E 13

3. $0 \mathrm{E} \quad 13$

3.8 E 13

4. $6 \mathrm{E} 13$

$5.7 \mathrm{E} 13$

$6.7 \mathrm{E} 13$

8. $0 \mathrm{E} 13$

$9.4 \mathrm{E} \quad 13$

1. $1 \mathrm{E} 14$

1. $3 \mathrm{E} 14$

$1.4 \mathrm{E} 14$

1. $6 \mathrm{E} 14$

1. $8 \mathrm{E} 14$

2. $8 \mathrm{E} 05$

7. $8 \mathrm{E} 05$ 5. $4 \mathrm{E} 07$ 2. $1 \mathrm{E} 08$ 3. 8 E 08 5. $6 \mathrm{E} 08$ 8.2 E 08 1. 2 E 09 1. $6 \mathrm{E} 09$ 2. 0 E 09 2. 5 E 09 3. $1 \mathrm{E} 09$ 3. 8 E 09 4. $5 \mathrm{E} \quad 09$ 5. $3 \mathrm{E} 09$ 6. $3 \mathrm{E} 09$ 7. $3 \mathrm{E} 09$ $8.4 \mathrm{E} 09$ $9.6 \mathrm{E} 09$ 1. $1 \mathrm{E} 10$ 1. $2 \mathrm{E} 10$ 1. $3 \mathrm{E} 10$
1. 9 E 14
100

300

1000

3000

10000

$\begin{array}{ll}999.6 & 0.14 \\ 999.6 & 0.00 \\ 999.6 & 0.00 \\ 999.6 & 0.00 \\ 999.6 & 0.0\end{array}$

$\stackrel{\infty}{\longrightarrow}$


are given in Table 4.58. Table 4.59 presents the annual and accumulated amounts of compacted, solid non-TRU wastes from all fuel cycle sources (including reactors) at surface burial grounds.

\subsection{Ore Tailings}

The processes used to extract uranium from ore are based on either alkaline or acid leaching followed by recovery of the uranium from the leach liquors by solvent extraction or ion exchange techniques. ${ }^{36}$ The ore typically averages about $0.2 \%$ uranium as $\mathrm{U}_{3} \mathrm{O}_{8}$, and the mills normally recover about $95 \%$ of it. The remaining $5 \%$ is discharged in the wastes, or tailings, with the other naturally occurring radioisotopes originally present in the ore. The tailings consist of sands, slimes containing the bulk of the radioactivity, and process leach liquors. They are discharged to tailings ponds adjacent to the mills from which the liquids either evaporate or seep into the ground. The solids, which are roughly equivalent in volume to the original ore, average about $0.16 \mathrm{yd}^{3}$ per pound of recovered $\mathrm{U}_{3} \mathrm{O}_{8}$. Their future disposition remains in doubt; however, it is likely that they will be returned to the mines from which they came or will be covered in place with plastic or asphaltic membranes and earth to reduce dispersal by weathering.

Thorium ores are not as yet processed on a large scale in the U.S., but for this study we consider them comparable to uranium ores in milling and tailings characteristics.

The accumulations of tailings from milling uranium and thorium ores are presented in Tables 4.60 and 4.61 . These projections are based on the assumption that $95 \%$ of the uranium and thorium is extracted from ores that assay $0.2 \%$ uranium or thorium. The unrecovered $5 \%$ and all radioactive daughters remain with the tailings. The volume of tailings is estimated on the basis of $0.16 \mathrm{yd}^{3}$ being formed per pound of recovered $\mathrm{U}_{3} \mathrm{O}_{8}$ or $\mathrm{ThO}_{2}$. 
Eable 4.58. Profected annual accumulation of low-level nontransuranic waste from reactors

\begin{tabular}{|c|c|c|c|c|c|}
\hline Year & $\begin{array}{l}\text { * * } \\
\text { volume } \\
\text { (nilizions } \\
\left.\text { of } \text { of }^{3}\right)\end{array}$ & $\begin{array}{l}\text { Annual a } \\
\text { Radio- } \\
\text { activity } \\
\text { (HCi) }\end{array}$ & $\begin{array}{c}\text { ddition } \\
\text { Thermal } \\
\text { Pover } \\
\text { (kn) }\end{array}$ & $\begin{array}{c}* * \\
\text { Burial } \\
\text { Area } \\
\text { (Acres) }\end{array}$ & $\begin{array}{l}\text { Annual } \\
\text { Number } \\
\text { of } \\
\text { Shi pments }\end{array}$ \\
\hline $\begin{array}{l}1971 \\
1972 \\
1973 \\
1974 \\
1975 \\
1976 \\
1977 \\
1978 \\
1979 \\
1980 \\
1981 \\
1982 \\
1983 \\
1984 \\
1985 \\
1986 \\
1987 \\
1988 \\
1989 \\
1990 \\
1991 \\
1992 \\
1993 \\
1994 \\
1995 \\
1996 \\
1997 \\
1998 \\
1999 \\
2000 \\
2001\end{array}$ & $\begin{array}{l}0.002 \\
C .003 \\
0.004 \\
C .006 \\
0.010 \\
0.014 \\
0.019 \\
0.021 \\
0.024 \\
0.026 \\
0.029 \\
0.033 \\
0.038 \\
0.045 \\
0.054 \\
0.062 \\
0.071 \\
0.079 \\
0.088 \\
0.098 \\
0.107 \\
0.1177 \\
0.126 \\
0.137 \\
0.147 \\
0.157 \\
0.168 \\
0.179 \\
0.191 \\
0.204 \\
0.217\end{array}$ & $\begin{array}{l}0.00 \\
0.00 \\
0.01 \\
0.01 \\
0.01 \\
0.02 \\
0.03 \\
0.03 \\
0.03 \\
0.04 \\
0.04 \\
0.05 \\
0.05 \\
0.06 \\
0.08 \\
0.18 \\
0.20 \\
0.22 \\
0.25 \\
0.27 \\
0.45 \\
0.49 \\
0.53 \\
0.58 \\
0.62 \\
0.57 \\
0.71 \\
0.76 \\
0.81 \\
0.86 \\
0.92\end{array}$ & $\begin{array}{l}0.01 \\
0.01 \\
0.02 \\
0.03 \\
0.04 \\
0.06 \\
0.08 \\
0.09 \\
0.10 \\
0.11 \\
0.12 \\
0.14 \\
0.16 \\
0.19 \\
0.22 \\
0.52 \\
0.50 \\
0.66 \\
0.73 \\
0.81 \\
1.32 \\
1.45 \\
1.57 \\
1.65 \\
1.82 \\
1.95 \\
2.08 \\
2.22 \\
2.37 \\
2.52 \\
2.68\end{array}$ & $\begin{array}{r}1.5 \\
2.4 \\
3.0 \\
4.5 \\
6.9 \\
9.6 \\
13.2 \\
14.8 \\
16.7 \\
18.0 \\
20.4 \\
23.6 \\
27.1 \\
31.8 \\
38.0 \\
44.0 \\
50.0 \\
56.0 \\
62.3 \\
68.9 \\
75.5 \\
82.4 \\
89.3 \\
96.5 \\
103.7 \\
111.2 \\
118.7 \\
126.5 \\
134.8 \\
143.8 \\
153.0\end{array}$ & $\begin{array}{r}158 \\
253 \\
316 \\
474 \\
726 \\
1014 \\
1389 \\
1563 \\
1759 \\
1898 \\
2151 \\
2485 \\
2858 \\
3344 \\
4001 \\
4636 \\
5267 \\
5899 \\
6562 \\
7257 \\
7952 \\
8678 \\
9404 \\
10162 \\
10920 \\
11709 \\
12499 \\
13320 \\
14195 \\
15142 \\
16108\end{array}$ \\
\hline
\end{tabular}

* * * Accumulation Through End of Year * * * * * Volume Radio- Thermal Burial Hazard, Cubic $\begin{array}{llll}\text { (Millions activity Power } & \text { Area } & \text { Meters at RCG } \\ \text { of } \mathbb{B}^{3} \text { ) } & \text { (MCi) } & \text { (kw) } & \text { (ACres) Air }\end{array}$

$\begin{array}{llrr}0.002 & 0.00 & 0.0 & 1 \\ 0.006 & 0.01 & 0.0 & 4 \\ 0.010 & 0.01 & 0.0 & 7 \\ 0.016 & 0.02 & 0.1 & 11 \\ 0.026 & 0.03 & 0.1 & 18 \\ 0.040 & 0.04 & 0.1 & 28 \\ 0.058 & 0.06 & 0.2 & 41 \\ 0.079 & 0.07 & 0.2 & 56 \\ 0.103 & 0.09 & 0.3 & 73 \\ 0.128 & 0.11 & 0.3 & 91 \\ 0.157 & 0.12 & 0.4 & 111 \\ 0.191 & 0.14 & 0.4 & 135 \\ 0.229 & 0.17 & 0.5 & 162 \\ 0.274 & 0.19 & 0.6 & 194 \\ 0.328 & 0.23 & 0.7 & 232 \\ 0.390 & 0.35 & 1.0 & 276 \\ 0.461 & 0.47 & 1.4 & 326 \\ 0.541 & 0.59 & 1.7 & 382 \\ 0.629 & 0.71 & 2.1 & 444 \\ 0.726 & 0.83 & 2.4 & 513 \\ 0.833 & 1.10 & 3.2 & 589 \\ 0.950 & 1.35 & 3.9 & 671 \\ 1.077 & 1.59 & 4.6 & 760 \\ 1.213 & 1.82 & 5.3 & 857 \\ 1.360 & 2.04 & 5.9 & 961 \\ 1.518 & 2.26 & 6.6 & 1072 \\ 1.686 & 2.48 & 7.2 & 1191 \\ 1.865 & 2.70 & 7.8 & 1317 \\ 2.056 & 2.92 & 8.5 & 1452 \\ 2.260 & 3.15 & 9.1 & 1596 \\ 2.476 & 3.39 & 9.8 & 1749\end{array}$

2. $1 \mathrm{E} \quad 12 \quad 2.7 \mathrm{E} \quad 07$ $\begin{array}{llll}5.0 \mathrm{E} & 12 & 6.4 \mathrm{E} & 07\end{array}$ 8. $1 \mathrm{E} \quad 12 \quad 1.1 \mathrm{E} \quad 08$ 1. 3 E 13 1.7E 08 2.0 13 E 08 . $1.8 \mathrm{E} 08$ 5. $1 \mathrm{E} 13 \quad 5.4 \mathrm{E} 08$ 5. $3 \mathrm{E} 13 \quad 7.0 \mathrm{E} 08$ $6.5 \mathrm{E} 13 \quad 8.7 \mathrm{E} 08$ $1.7 \mathrm{E} 13 \quad 1.0 \mathrm{E} 09$ 8. $9 \mathrm{E} 13 \quad 1.2 \mathrm{E} 09$ $1.0 \mathrm{E} 14 \quad 1.4 \mathrm{E} 09$ $\begin{array}{llll}1.2 \mathrm{E} & 14 & 1.6 \mathrm{E} & 09\end{array}$ $1.4 \mathrm{E} 14$ 1.9E 09 $\begin{array}{llll}1.6 \mathrm{E} & 14 & 2.2 \mathrm{E} & 09\end{array}$ $2.5 \mathrm{E} 14 \quad 3.4 \mathrm{E} \quad 09$ $3.4 \mathrm{E} 14 \quad 4.6 \mathrm{E} \quad 09$ $\begin{array}{llll}4.2 \mathrm{E} & 14 & 5.7 \mathrm{E} & 09\end{array}$ $\begin{array}{lllll}5.1 \mathrm{E} & 14 & 6.9 \mathrm{E} & 09\end{array}$ 5. $9 \mathrm{E} \quad 14 \quad 8.1 \mathrm{E} 09$ $\begin{array}{lllll}7.9 \mathrm{E} & 14 & 1.1 \mathrm{E} & 10\end{array}$ 9.7 E $14 \quad 1.3$ E 10 1. $1 \mathrm{E} 15 \quad 15 \mathrm{E} 10$ $1.3 \mathrm{E} 151.8 \mathrm{E} 10$ $1.5 \mathrm{E} 152.0 \mathrm{D} 10$ $1.5 \mathrm{E} 15$ 2.0E 10 $1.6 \mathrm{E} 15 \quad 2.2 \mathrm{E} 10$ $1.8 \mathrm{E} 15 \mathrm{2} 2.5 \mathrm{E} 10$ 1.9. 15 $2.7 \mathrm{E} 10$ 2.1E $15 \quad 3.0 \mathrm{E} 10$ $\begin{array}{llll}2.3 \mathrm{E} & 15 & 3.2 \mathrm{E} & 10 \\ 2.4 \mathrm{E} & 15 & 3.5 \mathrm{E} & 10\end{array}$

TIME AFTER SHOTDOHN, YEARS

100
300
1000
3000
10000
30000
100000
300000
1000000

$\begin{array}{llll}2.476 & 0.11 & 0.0 & 1749 \\ 2.476 & 0.02 & 0.0 & 1749 \\ 2.476 & 0.00 & 0.0 & 1749 \\ 2.476 & 0.00 & 0.0 & 1749 \\ 2.476 & 0.00 & 0.0 & 1749 \\ 2.476 & 0.00 & 0.0 & 1749 \\ 2.476 & 0.00 & 0.0 & 1749 \\ 2.476 & 0.00 & 0.0 & 1749 \\ 2.476 & 0.00 & 0.0 & 1749\end{array}$


Table 4.59. Profected annul accumulation of low-level nontransuranic waste at surface burial grounds

\begin{tabular}{|c|c|c|c|c|c|}
\hline ear & $\begin{array}{l}\text { * * * } \\
\text { volume } \\
\text { (nillions } \\
\text { of } \text { (1) }^{3}\end{array}$ & $\begin{array}{l}\text { Annual Ad } \\
\text { Badio- } \\
\text { activity } \\
\text { (nCi) }\end{array}$ & $\begin{array}{l}\text { ddition } \\
\text { Therwal } \\
\text { Power } \\
\text { (kH) }\end{array}$ & $\begin{array}{c}\text { * } * * \\
\text { Burial } \\
\text { Area } \\
\text { (Acres) }\end{array}$ & $\begin{array}{c}\text { Annual } \\
\text { Number } \\
\text { of } \\
\text { Shipgent }\end{array}$ \\
\hline $\begin{array}{l}1971 \\
1972 \\
1973 \\
1974 \\
1975 \\
1976 \\
1977 \\
1978 \\
1979 \\
1980 \\
1981 \\
1982 \\
1983 \\
1984 \\
1985 \\
1986 \\
1987 \\
1988 \\
1989 \\
1990 \\
1991 \\
1992 \\
1993 \\
1994 \\
1995 \\
1996 \\
1997 \\
1998 \\
1999 \\
2000 \\
2001\end{array}$ & $\begin{array}{l}0.002 \\
0.003 \\
0.004 \\
0.006 \\
0.010 \\
0.014 \\
0.019 \\
0.021 \\
0.024 \\
0.026 \\
0.029 \\
0.034 \\
0.043 \\
0.053 \\
0.067 \\
0.077 \\
0.089 \\
0.092 \\
0.097 \\
0.109 \\
0.125 \\
0.140 \\
0.153 \\
0.163 \\
0.171 \\
0.186 \\
0.203 \\
0.219 \\
0.235 \\
0.250 \\
0.264\end{array}$ & $\begin{array}{l}0.00 \\
0.00 \\
0.01 \\
0.01 \\
0.01 \\
0.02 \\
0.03 \\
0.03 \\
0.03 \\
0.04 \\
0.04 \\
0.05 \\
0.05 \\
0.06 \\
0.08 \\
0.18 \\
0.20 \\
0.22 \\
0.25 \\
0.28 \\
0.45 \\
0.50 \\
0.54 \\
0.58 \\
0.62 \\
0.67 \\
0.71 \\
0.76 \\
0.81 \\
0.87 \\
0.92\end{array}$ & $\begin{array}{l}0.01 \\
0.01 \\
0.02 \\
0.03 \\
0.04 \\
0.06 \\
0.08 \\
0.09 \\
0.10 \\
0.11 \\
0.12 \\
0.14 \\
0.16 \\
0.19 \\
0.23 \\
0.52 \\
0.59 \\
0.66 \\
0.73 \\
0.81 \\
1.33 \\
1.46 \\
1.58 \\
1.71 \\
1.83 \\
1.97 \\
2.10 \\
2.24 \\
2.39 \\
2.55 \\
2.71\end{array}$ & $\begin{array}{r}1.5 \\
2.4 \\
3.0 \\
4.5 \\
6.9 \\
9.6 \\
13.2 \\
14.8 \\
16.7 \\
18.0 \\
20.4 \\
24.0 \\
30.0 \\
37.4 \\
47.6 \\
54.4 \\
57.4 \\
65.0 \\
68.4 \\
77.3 \\
88.2 \\
99.2 \\
108.1 \\
115.5 \\
120.9 \\
131.5 \\
143.7 \\
154.6 \\
165.7 \\
176.6 \\
186.2\end{array}$ & $\begin{array}{r}158 \\
253 \\
316 \\
474 \\
726 \\
1014 \\
1389 \\
1563 \\
1759 \\
1898 \\
2151 \\
2527 \\
3160 \\
3935 \\
5007 \\
5729 \\
6040 \\
6847 \\
7204 \\
8132 \\
9288 \\
10443 \\
11376 \\
12153 \\
12725 \\
13845 \\
15128 \\
16277 \\
17439 \\
18588 \\
19597\end{array}$ \\
\hline
\end{tabular}

\begin{tabular}{|c|c|c|c|c|c|c|c|}
\hline $\begin{array}{l}\text { Volume } \\
\text { (Millions } \\
\text { of m } 3 \text { ) }\end{array}$ & $\begin{array}{l}\text { Radio- } \\
\text { activity } \\
\text { (MCi) }\end{array}$ & $\begin{array}{c}\text { Thermal } \\
\text { Pover } \\
\text { (kw) }\end{array}$ & $\begin{array}{l}\text { Burial } \\
\text { Area } \\
\text { (Acres) }\end{array}$ & & $\begin{array}{l}\text { zard. } \\
\text { ters }\end{array}$ & $\begin{array}{r}\text { Cubic } \\
\text { at RCG } \\
\text { Water }\end{array}$ & \\
\hline $\begin{array}{l}0.002 \\
0.006 \\
0.010 \\
0.016 \\
0.026 \\
0.040 \\
0.058 \\
0.079 \\
0.103 \\
0.128 \\
0.157 \\
0.191 \\
0.234 \\
0.287 \\
0.354 \\
0.431 \\
0.512 \\
0.605 \\
0.701 \\
0.811 \\
0.936 \\
1.076 \\
1.229 \\
1.393 \\
1.564 \\
1.750 \\
1.954 \\
2.177 \\
2.407 \\
2.657 \\
2.321\end{array}$ & $\begin{array}{l}0.00 \\
0.01 \\
0.01 \\
0.02 \\
0.03 \\
0.04 \\
0.06 \\
0.07 \\
0.09 \\
0.11 \\
0.12 \\
0.14 \\
0.17 \\
0.19 \\
0.23 \\
0.35 \\
0.48 \\
0.60 \\
0.71 \\
0.83 \\
1.10 \\
1.36 \\
1.59 \\
1.83 \\
2.05 \\
2.27 \\
2.49 \\
2.71 \\
2.94 \\
3.17 \\
3.41\end{array}$ & $\begin{array}{l}0.0 \\
0.0 \\
0.0 \\
0.1 \\
0.1 \\
0.1 \\
0.2 \\
0.2 \\
0.3 \\
0.3 \\
0.4 \\
0.4 \\
0.5 \\
0.6 \\
0.7 \\
1.0 \\
1.4 \\
1.7 \\
2.1 \\
2.4 \\
3.2 \\
4.0 \\
4.6 \\
5.3 \\
6.0 \\
6.6 \\
7.3 \\
7.9 \\
8.55 \\
9.2 \\
9.9\end{array}$ & $\begin{array}{r}1 \\
4 \\
7 \\
11 \\
18 \\
28 \\
41 \\
56 \\
73 \\
71 \\
111 \\
135 \\
165 \\
203 \\
250 \\
305 \\
362 \\
427 \\
495 \\
573 \\
661 \\
760 \\
868 \\
984 \\
1105 \\
1236 \\
1380 \\
1534 \\
1700 \\
1877 \\
2063\end{array}$ & $\begin{array}{l}2.1 \mathrm{E} \\
5.0 \mathrm{Q} \\
8.1 \mathrm{E} \\
1.3 \mathrm{E} \\
2.0 \mathrm{E} \\
2.9 \mathrm{E} \\
4.1 \mathrm{E} \\
5.3 \mathrm{E} \\
6.5 \mathrm{E} \\
7.7 \mathrm{E} \\
8.9 \mathrm{E} \\
1.0 \mathrm{E} \\
1.2 \mathrm{P} \\
1.4 \mathrm{E} \\
1.7 \mathrm{E} \\
2.6 \mathrm{E} \\
3.5 \mathrm{E} \\
4.3 \mathrm{E} \\
5.2 \mathrm{E} \\
6.1 \mathrm{E} \\
8.1 \mathrm{P} \\
9.9 \mathrm{E} \\
1.2 \mathrm{E} \\
1.3 \mathrm{E} \\
1.5 \mathrm{E} \\
1.7 \mathrm{E} \\
1.8 \mathrm{E} \\
2.0 \mathrm{E} \\
2.2 \mathrm{E} \\
2.4 \mathrm{Z} \\
2.5 \mathrm{E}\end{array}$ & $\begin{array}{l}12 \\
12 \\
12 \\
13 \\
13 \\
13 \\
13 \\
13 \\
13 \\
13 \\
13 \\
14 \\
14 \\
14 \\
14 \\
14 \\
14 \\
14 \\
14 \\
14 \\
14 \\
14 \\
15 \\
15 \\
15 \\
15 \\
15 \\
15 \\
15 \\
15 \\
15\end{array}$ & $\begin{array}{l}2.7 \mathrm{E} \\
6.4 \mathrm{E} \\
1.1 \mathrm{E} \\
1.7 \mathrm{E} \\
2.6 \mathrm{E} \\
3.9 \mathrm{E} \\
5.4 \mathrm{E} \\
7.0 \mathrm{E} \\
8.7 \mathrm{E} \\
1.0 \mathrm{E} \\
1.2 \mathrm{E} \\
1.4 \mathrm{E} \\
1.7 \mathrm{E} \\
2.0 \mathrm{E} \\
2.5 \mathrm{E} \\
3.3 \mathrm{E} \\
5.2 \mathrm{E} \\
6.5 \mathrm{E} \\
7.9 \mathrm{E} \\
9.3 \mathrm{E} \\
1.2 \mathrm{E} \\
1.5 \mathrm{E} \\
1.8 \mathrm{E} \\
2.1 \mathrm{E} \\
2.4 \mathrm{E} \\
2.7 \mathrm{E} \\
3.0 \mathrm{E} \\
3.3 \mathrm{E} \\
3.6 \mathrm{E} \\
3.9 \mathrm{E} \\
4.3 \mathrm{E}\end{array}$ & $\begin{array}{l}07 \\
07 \\
08 \\
08 \\
08 \\
08 \\
08 \\
09 \\
09 \\
09 \\
09 \\
09 \\
09 \\
09 \\
09 \\
09 \\
09 \\
09 \\
09 \\
09 \\
10 \\
10 \\
10 \\
10 \\
10 \\
10 \\
10 \\
10 \\
10 \\
10 \\
10\end{array}$ \\
\hline $\begin{array}{l}2.921 \\
2.921 \\
2.921 \\
2.921 \\
2.921 \\
2.921 \\
2.921 \\
2.921 \\
2.921\end{array}$ & $\begin{array}{l}0.11 \\
0.02 \\
0.00 \\
0.00 \\
0.00 \\
0.00 \\
0.00 \\
0.00 \\
0.00\end{array}$ & $\begin{array}{l}0.0 \\
0.0 \\
0.0 \\
0.0 \\
0.0 \\
0.0 \\
0.0 \\
0.0 \\
0.0\end{array}$ & $\begin{array}{l}2063 \\
2063 \\
2063 \\
2063 \\
2063 \\
2063 \\
2063 \\
2063 \\
2063\end{array}$ & $\begin{array}{l}5.9 \mathrm{E} \\
1.2 \mathrm{E} \\
1.6 \mathrm{E} \\
9.9 \mathrm{E} \\
9.4 \mathrm{E} \\
8.2 \mathrm{E} \\
5.4 \mathrm{E} \\
2.6 \mathrm{E} \\
1.4 \mathrm{E}\end{array}$ & $\begin{array}{l}13 \\
13 \\
11 \\
10 \\
10 \\
10 \\
10 \\
10 \\
10\end{array}$ & $\begin{array}{l}4.1 \mathrm{E} \\
7.8 \mathrm{E} \\
1.2 \mathrm{E} \\
7.6 \mathrm{E} \\
7.1 \mathrm{E} \\
6.0 \mathrm{E} \\
3.3 \mathrm{E} \\
7.3 \mathrm{E} \\
1.2 \mathrm{E}\end{array}$ & $\begin{array}{l}09 \\
08 \\
07 \\
06 \\
06 \\
06 \\
06 \\
05 \\
05 \\
05\end{array}$ \\
\hline
\end{tabular}


Table 4.60. Profected annual accumulation of uranium ore tailings

* * * An nual Addition ***** * Tolume Heary Badio- Therma Year Millions Element activity Power (ICi) (kw)

$\begin{array}{lrrrr}1970 & 0.82 & 132 & 0.01 & 0.21 \\ 1971 & 0.77 & 125 & 0.01 & 0.20 \\ 1972 & 1.43 & 231 & 0.02 & 0.36 \\ 1973 & 2.28 & 369 & 0.03 & 0.58 \\ 1974 & 2.73 & 442 & 0.03 & 0.70 \\ 1975 & 3.58 & 578 & 0.04 & 0.91 \\ 1976 & 2.93 & 474 & 0.03 & 0.75 \\ 1977 & 3.26 & 526 & 0.04 & 0.83 \\ 1978 & 3.22 & 521 & 0.04 & 0.82 \\ 1979 & 4.16 & 673 & 0.05 & 1.06 \\ 1980 & 4.99 & 807 & 0.06 & 1.27 \\ 1981 & 5.48 & 886 & 0.06 & 1.40 \\ 1982 & 6.57 & 1062 & 0.08 & 1.67 \\ 1983 & 8.02 & 1296 & 0.09 & 2.04 \\ 1984 & 8.71 & 1407 & 0.10 & 2.22 \\ 1985 & 9.47 & 1530 & 0.11 & 2.41 \\ 1986 & 10.16 & 1643 & 0.12 & 2.59 \\ 1987 & 11.24 & 1816 & 0.13 & 2.86 \\ 1988 & 12.14 & 1963 & 0.14 & 3.09 \\ 1989 & 12.35 & 1996 & 0.14 & 3.15 \\ 1990 & 12.96 & 2095 & 0.15 & 3.30 \\ 1991 & 13.53 & 2187 & 0.16 & 3.45 \\ 1992 & 14.58 & 2357 & 0.17 & 3.72 \\ 1993 & 15.81 & 2555 & 0.18 & 4.03 \\ 1994 & 16.54 & 2674 & 0.19 & 4.22 \\ 1995 & 16.85 & 2724 & 0.20 & 4.29 \\ 1996 & 17.23 & 2785 & 0.20 & 4.39 \\ 1997 & 18.00 & 2909 & 0.21 & 4.59 \\ 1998 & 18.90 & 3054 & 0.22 & 4.82 \\ 1999 & 19.78 & 3197 & 0.23 & 5.04 \\ 2000 & 20.26 & 3274 & 0.24 & 5.16\end{array}$

TIHE AFTER SHOTDOHN, YEARS

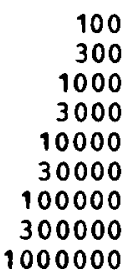

* * * Accumulation Through End of Year *\# * * *

Volume Heavy Radio- Thermal Hazard, Cubic $\begin{array}{llll}\text { (Billions Elements activity Power } & \text { Heters at RCG } \\ \text { of } \mathrm{m}^{3} \text { ) (MT) } & \text { (MCi) } & \text { (kM) } & \text { Air Hater }\end{array}$

$\begin{array}{lrrrrrrr}0.001 & 132 & 0.0 & 0.2 & 1.3 \mathrm{E} & 16 & 4.0 \mathrm{E} & 10 \\ 0.002 & 257 & 0.0 & 0.4 & 2.5 \mathrm{E} & 16 & 7.7 \mathrm{E} & 10 \\ 0.003 & 487 & 0.0 & 0.8 & 4.8 \mathrm{E} & 16 & 1.5 \mathrm{E} & 11 \\ 0.005 & 857 & 0.1 & 1.3 & 8.4 \mathrm{E} & 16 & 2.6 \mathrm{E} & 11 \\ 0.008 & 1298 & 0.1 & 2.0 & 1.3 \mathrm{E} & 17 & 3.9 \mathrm{E} & 11 \\ 0.012 & 1877 & 0.1 & 2.9 & 1.8 \mathrm{E} & 17 & 5.6 \mathrm{E} & 11 \\ 0.015 & 2351 & 0.2 & 3.7 & 2.3 \mathrm{E} & 17 & 7.0 \mathrm{E} & 11 \\ 0.018 & 2877 & 0.2 & 4.5 & 2.8 \mathrm{E} & 17 & 8.6 \mathrm{E} & 11 \\ 0.021 & 3398 & 0.2 & 5.3 & 3.3 \mathrm{E} & 17 & 1.0 \mathrm{E} & 12 \\ 0.025 & 4071 & 0.3 & 6.3 & 4.0 \mathrm{E} & 17 & 1.2 \mathrm{E} & 12 \\ 0.030 & 4878 & 0.3 & 7.6 & 4.8 \mathrm{E} & 17 & 1.5 \mathrm{E} & 12 \\ 0.036 & 5764 & 0.4 & 9.0 & 5.7 \mathrm{E} & 17 & 1.7 \mathrm{E} & 12 \\ 0.042 & 6826 & 0.5 & 10.6 & 6.7 \mathrm{E} & 17 & 2.0 \mathrm{E} & 12 \\ 0.050 & 8122 & 0.6 & 12.6 & 8.0 \mathrm{E} & 17 & 2.4 \mathrm{E} & 12 \\ 0.059 & 9529 & 0.7 & 14.8 & 9.4 \mathrm{E} & 17 & 2.9 \mathrm{E} & 12 \\ 0.068 & 11059 & 0.8 & 17.2 & 1.1 \mathrm{E} & 18 & 3.3 \mathrm{E} & 12 \\ 0.079 & 12701 & 0.9 & 19.7 & 1.2 \mathrm{E} & 18 & 3.8 \mathrm{E} & 12 \\ 0.090 & 14517 & 1.0 & 22.5 & 1.4 \mathrm{E} & 18 & 4.4 \mathrm{E} & 12 \\ 0.102 & 16480 & 1.2 & 25.6 & 1.6 \mathrm{E} & 18 & 4.9 \mathrm{E} & 12 \\ 0.114 & 18476 & 1.3 & 28.7 & 1.8 \mathrm{E} & 18 & 5.5 \mathrm{E} & 12 \\ 0.127 & 20571 & 1.4 & 31.9 & 2.0 \mathrm{E} & 18 & 6.2 \mathrm{E} & 12 \\ 0.141 & 22758 & 1.6 & 35.3 & 2.2 \mathrm{E} & 18 & 6.8 \mathrm{E} & 12 \\ 0.155 & 25115 & 1.8 & 39.0 & 2.5 \mathrm{E} & 18 & 7.5 \mathrm{E} & 12 \\ 0.171 & 27669 & 1.9 & 43.0 & 2.7 \mathrm{E} & 18 & 8.3 \mathrm{E} & 12 \\ 0.188 & 30343 & 2.1 & 47.1 & 3.0 \mathrm{E} & 18 & 9.1 \mathrm{E} & 12 \\ 0.205 & 33067 & 2.3 & 51.3 & 3.3 \mathrm{E} & 18 & 9.9 \mathrm{E} & 12 \\ 0.222 & 35852 & 2.5 & 55.6 & 3.5 \mathrm{E} & 18 & 1.1 \mathrm{E} & 13 \\ 0.240 & 38761 & 2.7 & 60.1 & 3.8 \mathrm{E} & 18 & 1.2 \mathrm{E} & 13 \\ 0.259 & 41816 & 2.9 & 64.9 & 4.1 \mathrm{E} & 18 & 1.3 \mathrm{E} & 13 \\ 0.279 & 45012 & 3.2 & 69.8 & 4.4 \mathrm{E} & 18 & 1.3 \mathrm{E} & 13 \\ 0.299 & 48287 & 3.4 & 74.9 & 4.7 \mathrm{E} & 18 & 1.4 \mathrm{E} 13 \\ & & & & & & & \\ & & & & & & & \\ 0.299 & 48287 & 3.4 & 74.9 & 4.7 \mathrm{E} & 18 & 1.4 \mathrm{E} & 13 \\ 0.299 & 48287 & 3.4 & 74.9 & 4.7 \mathrm{E} & 18 & 1.4 \mathrm{E} & 13 \\ 0.299 & 48287 & 3.4 & 74.7 & 4.7 \mathrm{E} & 18 & 1.4 \mathrm{E} & 13 \\ 0.299 & 48287 & 3.3 & 73.9 & 4.6 \mathrm{E} & 18 & 1.4 \mathrm{E} & 13 \\ 0.299 & 48287 & 3.2 & 69.9 & 4.3 \mathrm{E} & 18 & 1.4 \mathrm{E} & 13 \\ 0.299 & 48287 & 2.7 & 59.1 & 3.6 \mathrm{E} & 18 & 1.2 \mathrm{E} & 13 \\ 0.299 & 48287 & 1.5 & 33.8 & 2.0 \mathrm{E} & 18 & 6.6 \mathrm{E} & 12 \\ 0.299 & 48287 & 0.5 & 9.8 & 5.4 \mathrm{E} & 17 & 1.8 \mathrm{E} & 12 \\ 0.299 & 48287 & 0.5 & 4.7 & 2.4 \mathrm{E} & 17 & 7.3 \mathrm{E} & 11 \\ & & & & & & & \end{array}$


Table 4.61. Projected anual accumulation of thorium ore tailings

* * * Annual Addition * * * * * Volume Heavy Radio- Thermal Year of 3 ) (ntemt activity Power

$1976 \quad 0.003 \quad 0.5$

1976

1977

1079

1980

1981

1982

1983

1984

1985

1986

1987

1988

1989

1990

1991

1992

1993

1994

1995

1996
1997

1998

1999

2000

$$
\begin{aligned}
& 0.5 \\
& 0.1 \\
& 0.1 \\
& 0.1 \\
& 0.1 \\
& 0.1 \\
& 0.1 \\
& 0.1 \\
& 0.1 \\
& 0.1 \\
& 0.1 \\
& 0.1 \\
& 0.1 \\
& 0.1 \\
& 0.1 \\
& 0.1 \\
& 0.1 \\
& 0.1 \\
& 1.7 \\
& 2.0 \\
& 3.5 \\
& 4.5 \\
& 5.1 \\
& 5.7 \\
& 6.4
\end{aligned}
$$

\begin{tabular}{|c|c|c|c|c|c|c|c|}
\hline $\begin{array}{l}\text { volume } \\
\text { (Hillions } \\
\text { of m3) }\end{array}$ & $\begin{array}{c}\text { Accumula } \\
\text { Heavy } \\
\text { Elements } \\
\text { (MT) }\end{array}$ & $\begin{array}{l}\text { tion Thr } \\
\text { Radio- } \\
\text { activity } \\
\text { (curies) }\end{array}$ & $\begin{array}{c}\text { Thermal } \\
\text { Power } \\
\text { (Watts) }\end{array}$ & $\begin{array}{l}\text { Haz } \\
\text { Met } \\
\text { Air }\end{array}$ & ard, & $\begin{array}{l}\text { Cubic } \\
\text { at RCG } \\
\text { Water }\end{array}$ & \\
\hline 0.00 & 1 & 4 & 0 & $1.4 \mathrm{E}$ & 12 & $3.8 \mathrm{E}$ & 07 \\
\hline 0.00 & 1 & 6 & 0 & $2.5 \mathrm{E}$ & 12 & 4. $1 \mathrm{E}$ & 07 \\
\hline 0.00 & 1 & 8 & 0 & $3.3 E$ & 12 & $4.4 \mathrm{E}$ & 07 \\
\hline 0.01 & 1 & 10 & 0 & $4.0 \mathrm{E}$ & 12 & $4.8 \mathrm{E}$ & 07 \\
\hline 0.01 & 1 & 11 & 0 & $4.6 \mathrm{E}$ & 12 & $5.1 \mathrm{E}$ & 07 \\
\hline 0.01 & 1 & 12 & 0 & $5.1 \mathrm{E}$ & 12 & $5.4 \mathrm{E}$ & 07 \\
\hline 0.01 & 1 & 13 & 0 & $5.6 \mathrm{E}$ & 12 & $5.7 \mathrm{E}$ & 07 \\
\hline 0.01 & 1 & 14. & 0 & $6.0 \mathrm{E}$ & 12 & $5.9 \mathrm{E}$ & 07 \\
\hline 0.01 & 1 & 15 & 0 & $6.3 \mathrm{E}$ & 12 & $6.1 \mathrm{E}$ & 07 \\
\hline 0.01 & 1 & 16 & 0 & $6.7 E$ & 12 & $6.3 E$ & 07 \\
\hline 0.01 & 2 & 16 & 0 & $7.0 \mathrm{E}$ & 12 & $6.5 \mathrm{E}$ & 07 \\
\hline 0.0 .1 & 2 & 17 & 0 & $7.2 \mathrm{E}$ & 12 & $6.7 \mathrm{E}$ & 07 \\
\hline 0.01 & 2 & 17 & 0 & $7.5 \mathrm{E}$ & 12 & $6.8 \mathrm{E}$ & 07 \\
\hline 0.01 & 2 & 18 & 0 & $7.7 \mathrm{E}$ & 12 & $7.0 \mathrm{E}$ & 07 \\
\hline $0: 01$ & 2 & 18 & 0 & $7.9 E$ & 12 & $7.1 \mathrm{E}$ & 07 \\
\hline 0.01 & 2 & 19 & 0 & $8.1 E$ & 12 & $7.2 \mathrm{E}$ & 07 \\
\hline 0.01 & 2 & 19 & 0 & 8. $3 E$ & 12 & 7. $3 \mathrm{E}$ & 07 \\
\hline 0.01 & 2 & 19 & 0 & $8.4 \mathrm{E}$ & 12 & $7.4 \mathrm{E}$ & 07 \\
\hline 0.03 & 4 & 32 & 1 & $1.3 \mathrm{E}$ & 13 & $1.9 \mathrm{E}$ & 08 \\
\hline 0.04 & 6 & 51 & 1 & $2.0 \mathrm{E}$ & 13 & 3. $1 \mathrm{E}$ & 08 \\
\hline 0.06 & 9 & 84 & 2 & 3. $3 \mathrm{E}$ & 13 & $5.2 \mathrm{E}$ & 08 \\
\hline 0.09 & 14 & 130 & 3 & $5.2 E$ & 13 & $7.9 \mathrm{E}$ & 08 \\
\hline 0.12 & 19 & 186 & 4 & $7.5 \mathrm{E}$ & 13 & $1.1 \mathrm{E}$ & 09 \\
\hline 0.15 & 25 & 251 & 5 & $1.0 \mathrm{E}$ & 14 & $1.4 \mathrm{E}$ & 09 \\
\hline 0.19 & 31 & 323 & 7 & 1. $3 \mathrm{E}$ & 14 & $1.7 \mathrm{E}$ & 09 \\
\hline
\end{tabular}$$
0.001
$$$$
0.001
$$$$
0.001
$$$$
0.001
$$$$
0.001
$$$$
0.001
$$$$
0.001
$$

4
1
1
1
1
1
1
1
1
1
1
1
1
1
1
1
1
1
13
15
26
34
38
43
48

TIME AFTER SHOTDOHN, YEARS

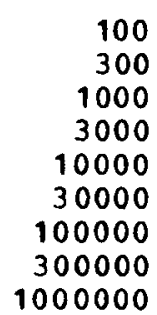

$\begin{array}{lll}0.19 & 31 & 34 \\ 0.19 & 31 & 34 \\ 0.19 & 31 & 34 \\ 0.19 & 31 & 34 \\ 0.19 & 31 & 34 \\ 0.19 & 31 & 34 \\ 0.19 & 31 & 34 \\ 0.19 & 31 & 34 \\ 0.19 & 31 & 34\end{array}$

$\begin{array}{llll}1.7 \mathrm{E} & 13 & 1.2 \mathrm{E} & 08\end{array}$ $1.6 \mathrm{E} 13 \quad 1.2 \mathrm{E} 08$ $\begin{array}{llll}1.6 \mathrm{E} & 13 & 1.2 \mathrm{E} & 08\end{array}$ $1.6 \mathrm{E} 13 \quad 1.2 \mathrm{E} 08$ $1.6 \mathrm{E} \quad 13 \quad 1.2 \mathrm{E} \quad 08$

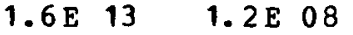

$\begin{array}{llll}1.6 \mathrm{E} & 13 & 1.2 \mathrm{E} & 08\end{array}$

$1.6 \mathrm{E} \quad 13 \quad 1.2 \mathrm{E} 08$

1. $6 \mathrm{E} 13 \quad 1.2 \mathrm{E} \quad 08$ 


\section{REFERENCES}

1. J. O. Blomeke, C. W. Kee, and J. P. Nichols, Projections of Radioactive Wastes to be Generated by the U.S. Nuclear Power Industry, ORNL/TM-3965 (1974).

2. E. J. Hanrahan, U.S. Energy Research and Development Administration, personal communication to F. P. Baranowski, ERDA, August 19, 1976.

3. Westinghouse Nuclear Energy Systems, RESAR-3, Reference Safety Analysis Report, DOCKET STN 50-480 (1972).

4. General Electric Standard Safety Analysis Report, BWR/6, DOCKET STN 50-447 (1973).

5. Pacific Northwest Laboratories, Theoretical Analysis of Plutonium Buildup and Uranium Depletion in Pressurized Water and Boiling Water Power Reactors, Report Y49024 to IAEA Division of Nuclear Power Reactors (July 1968).

6. L. J. Colby, R. C. Dahlberg, and S. Jaye, HTGR Fuel and Fuel Cycle Summary Description, GA-10233 (May 1971).

7. Keith E. Asmussen, Gulf General Atomic Company, unpublished mass flow data for reference 1160-MW(e) HTGR (August 1973).

8. T. D. Wolsko, Argonne National Laboratory, unpublished normalized mass flow data for the A. I. and G. E. Follow-On LMFBR designs (November 1969).

9. Oak Ridge National Laboratory, KWIKPLAN - A Code for Calculating Nuclear Fuel Cycle Mass Balances (unpublished).

10. Reactor Fuel Cycle Costs for Nuclear Power Evaluation, WASH-1099 (December 1971).

11. Energy Research and Development Administration, LWR Spent Fuel Disposition Capabilities, 1976-1985, ERDA-76-25 (May 1976).

12. M. J. Bell, ORIGEN - The ORNL Isotope Generation and Depletion Code, ORNL-4628 (May 1973).

13. "Siting of Fuel Reprocessing Plants and Related Waste Management Facilities," Federal Register 35, No. 222, 17530 (Nov. 14, 1970).

14. Staffs of the Oak Ridge National Laboratory and Gulf General Atomic, National H'I'GR Fuel Recycle Development Program Plan, ORNL-4702

(August 1971). 
15. L. Küchler, L. Schäffer, and B. Wottech, "The Thorex Two-Stage Process for Reprocessing Thorium Reactor Fuel with High Burnup," Kerntechnik 13 Jahrgang, No. 7/8 (1971).

16. J. W. Codding, W. O. Haas, and F. H. Heumann, Equilibrium Data for Purex Systems, KAPL-602 (November 1951).

17. Voloxidation--Removal of Volatile Fission Products from Spent LMFBR Fuels, J. H. Goode (compiler and editor), ORNL/TM-3723 (January 1973).

18. Alternatives for Managing Wastes from Reactors and Post-Fission Operations in the LWR Fuel Cycle, Volume 2, Chapter 6.0, ERDA-76-43 (May 1976).

19. Code of Federal Regulations, Title 10, Part 20, Appendix B, Table II.

20. J. J. Perona and J. O. Blomeke, A Parametric Study of Shipping Casks for Solid Radioactive Wastes, ORNL/TM-3651 (February 1972).

21. J. J. Perona, R. S. Dillon, and J. O. Blomeke, Design and Safety Considerations of Shipping Solidified High-Level Radioactive Wastes, ORNL/TM-2971 (December 1970).

22. G. R. Kilp, Westinghouse Electric Corp., Nuclear Fuel Division, Pittsburgh, Pa., personal communication (December 19, 1975).

23. Alternatives for Managing Wastes from Reactors and Post-Fission Operation in the LWR Fuel Cycle, Volume 2, Chapter 7.0, ERDA-76-43 (May 1976).

24. M. O. Marlowe, General Electric Company, San Jose, California, personal communication (February 18, 1976).

25. J. O. Blomeke and J. J. Perona, Storage, Shipment, and Disposal of Spent Fuel Cladding, ORNL/TM-3650 (January 1972).

26. H. W. Godbee and J. P. Nichols, Sources of Transuranium Solid Wastes and Their Influence on the Proposed National Radioactive Waste Repository, ORNL/TM-3277 (January 1971).

27. H. H. Van Tuyl et al., A Survey of Alpha Waste Generation and Disposal as Solids in the U.S. Nuclear Fuel Industry, BNWL-B-34 (December 1970).

28. E. P. McDonald, An Analysis of Waste Transportation Methods: The Shipment of Radioactive Waste Materials, Internal Report, Monsanto Research Corporation, Mound Laboratory (Facilities Engineering), Miamisburg, Ohio (April 10, 1970).

29. Alternatives for Managing Wastes from Reactors and Post-Fission Operations in the LWR Fuel Cycle, Volume 2, Chapter 13.0, ERDA-76-43 (May 1976). 
30. J. O. Blomeke and J. J. Perona, Management of Noble-Gas FissionProduct Wastes from Reprocessing Spent Fuels, ORNL/TM-2677

(Nov. 21, 1969).

31. W. E. Clark, C. T. Thompson, and W. B. Howerton, Fixation of Radioiodine with Portland Cement. I. Preliminary Scoping Studies, ORNL/TM-5064 (1975).

32. W. T. Crow, U.S. Nuclear Regulatory Commission, personal communication to L. C. Rouse, U.S. Nuclear Regulatory Commission (October 1974).

33. L. H. Brooks, C. A. Heath, B. Kirstein, and D. G. Roberts, Carbon-14 in the HTGR Fuel Cycle, GA-Al3174 (November 1974).

34. L. E. Trevorrow, B. J. Kullen, R. L. Jarry, and M. J. Steindler, Tritium and Noble-Gas Fission Products in the Nuclear Fuel Cycle. I. Reactors, ANL-8102 (1974).

35. A. H. Kibbey and H. W. Godbee, A Critical Review of Solid Radioactive Waste Practices at Nuclear Power Plants, ORNL-4924 (March 1974).

36. E. C. Tsivoglou and R. L. O'Connell, "Nature, Volume, and Activity of Uranium Mill Wastes," pp. 101-122 in Radiological Health and Safety in Mining and Milling of Nuclear Materials, Vol. II, IAEA, Vienna, 1964. 


\section{THIS PAGE}

\section{WAS INTENTIONALLY \\ LEFT BLANK}


APPENDIX A

Radioactivity and Thermal Power of High-Level Waste from One Metric Ton of Heavy Metal as a Function of Time for Six Reactor Types 
Table A.1. Curies of heavy isotopes in the high-level waste from one metric ton of uranium-enriched PWR fuel as a function of time

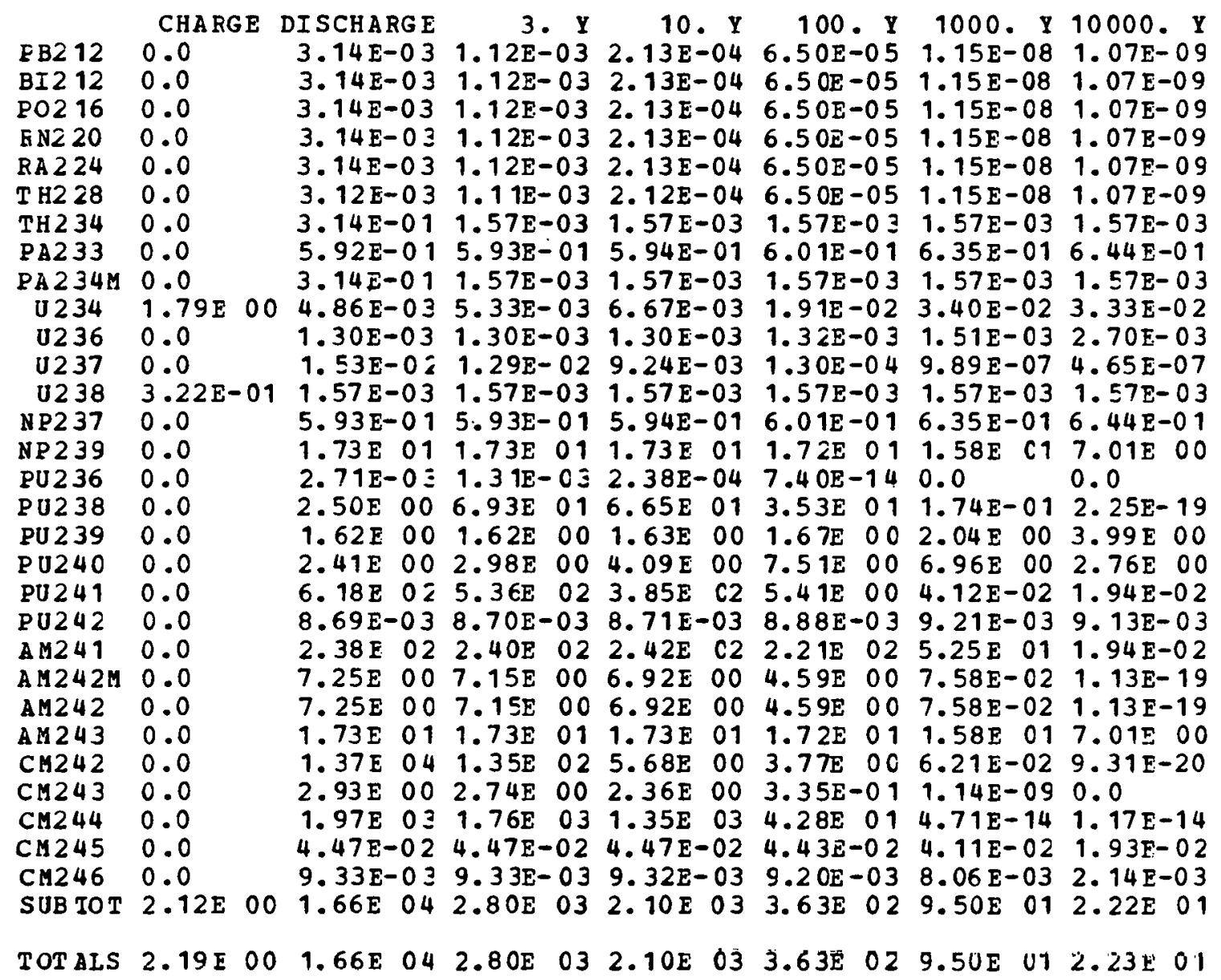


Table A.2. Curies of fission product isotopes in the high-level waste from one metric ton of uranium-enriched PWR fuel as a function of time

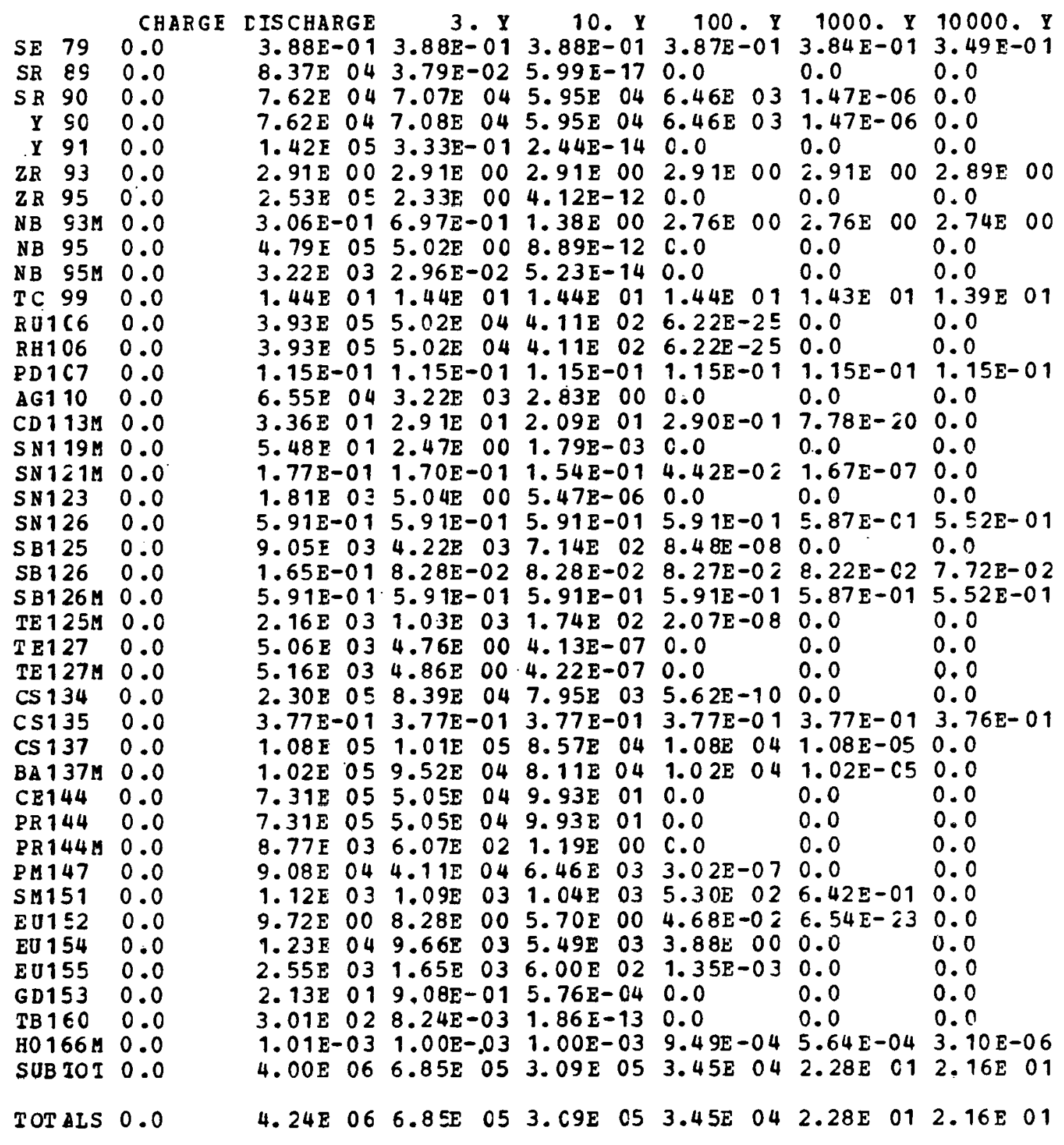


Table A.3. Curies of heavy isotopes in the high-level waste from one metric ton of plutonium-enriched PWR fuel as a function of time

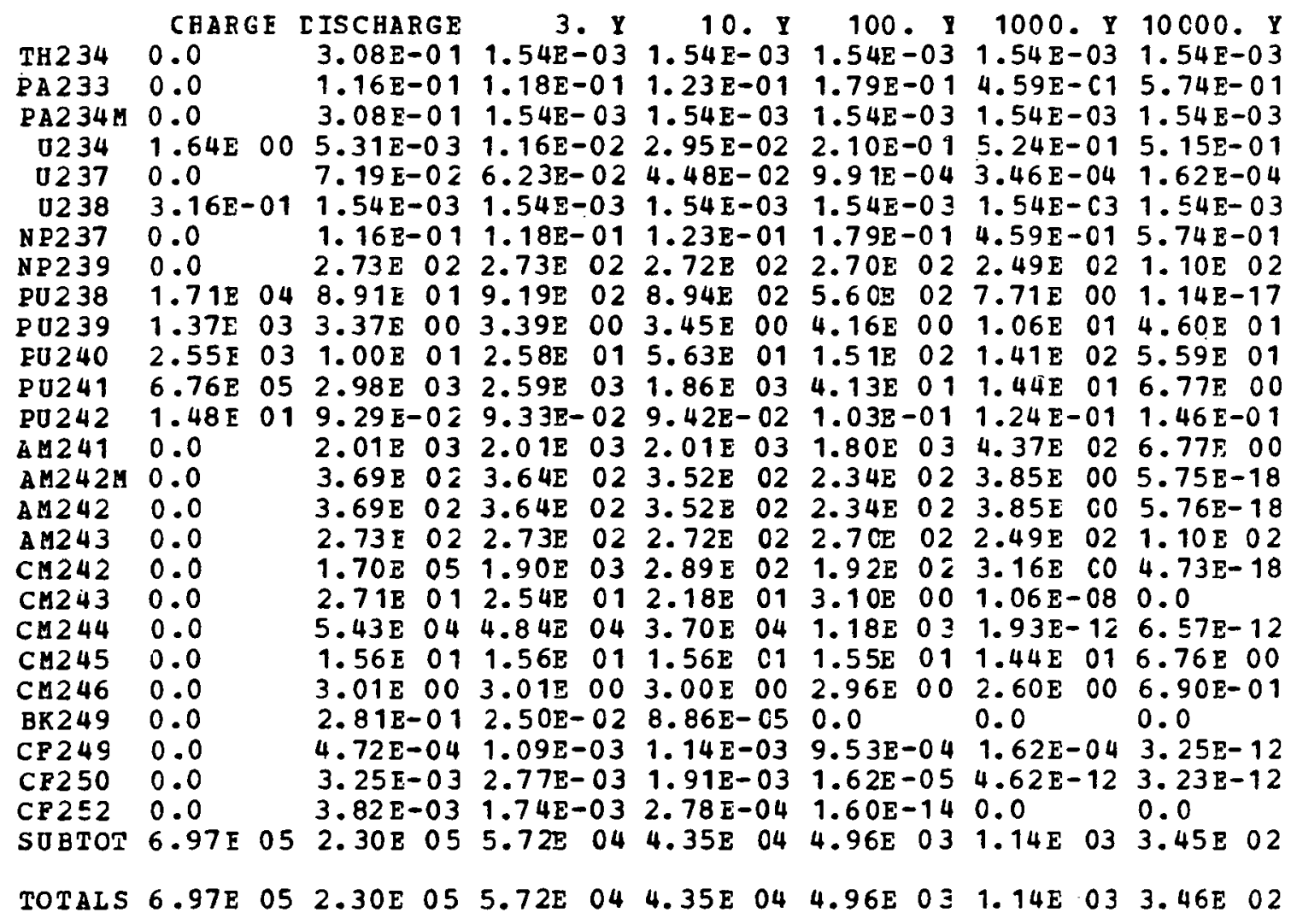


Table A.4. Curies of fission product isotopes in the high-level waste from one metric ton of plutonium-enriched PWR fuel as a function of time

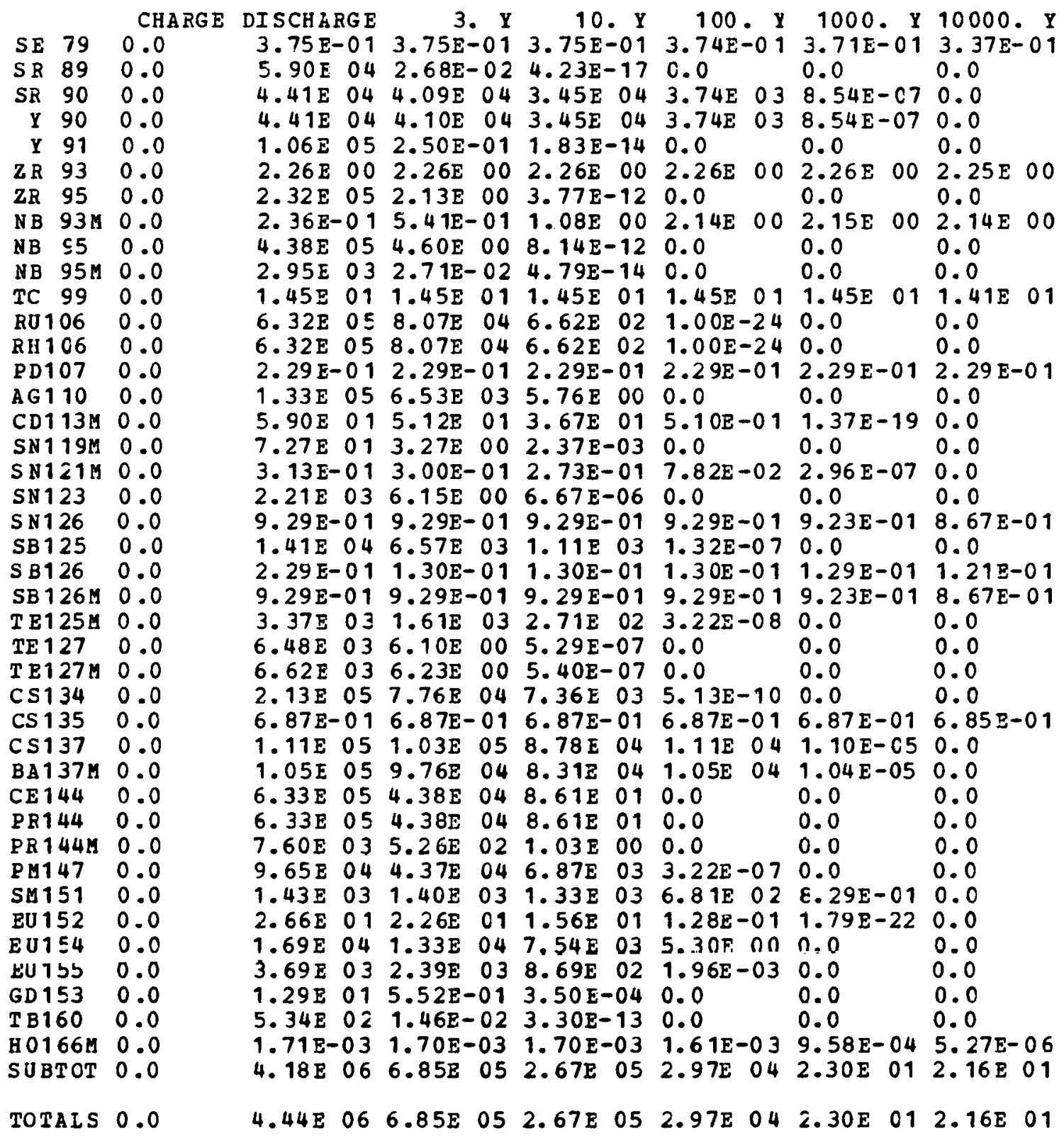


Table A.5. Curies of heavy isotopes in the high-level waste from one metric ton of uranium-enriched BWR fuel as a function of time

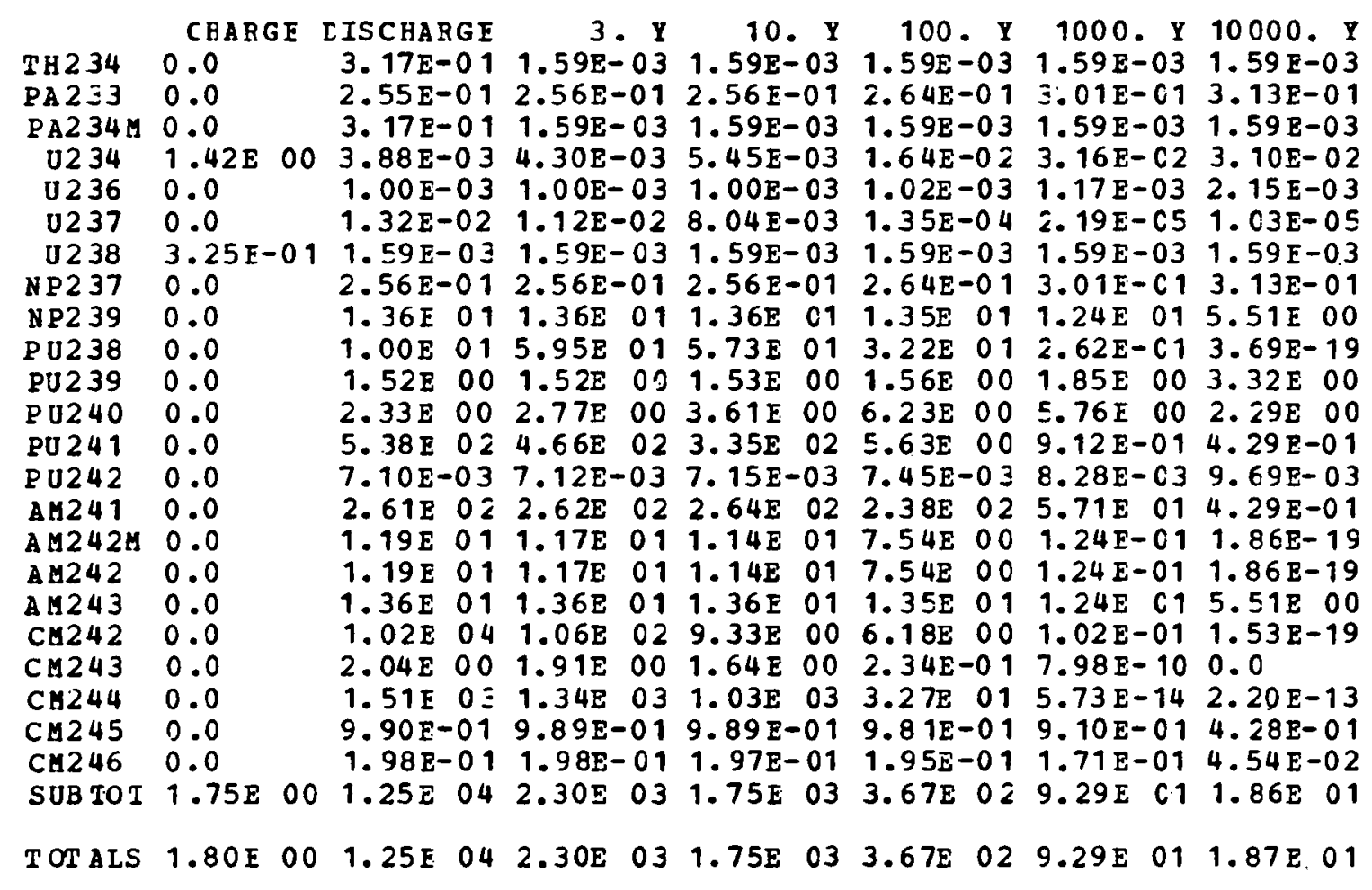


Table A.6. Curies of fission product isotopes in the high-level waste from one metric ton of uranium-enriched BWR fuel as a function of time

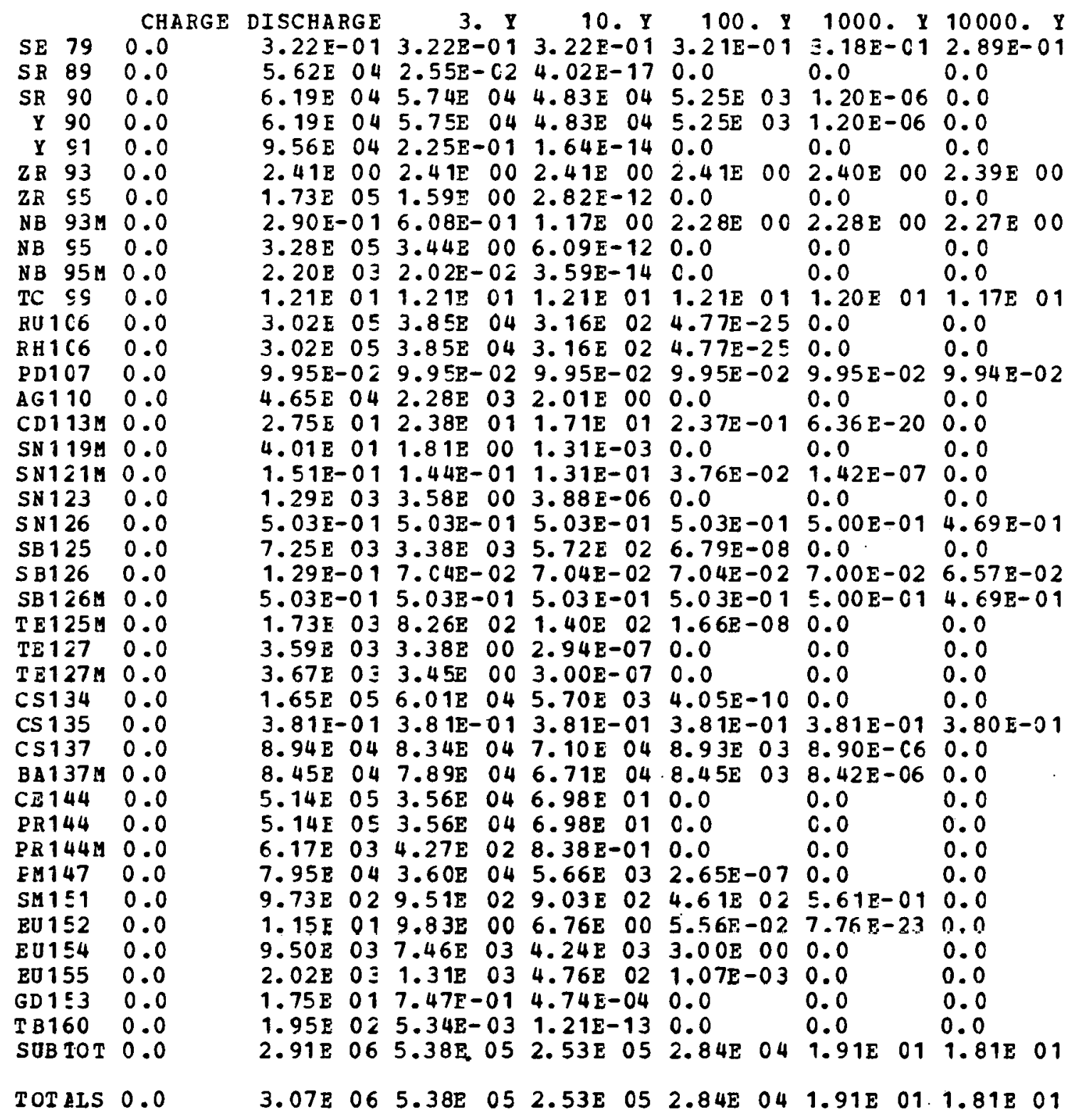


Table A.7. Curies of heavy isotopes in the high-level waste from one metric ton of plutonium-enriched BWR fuel as a function of time

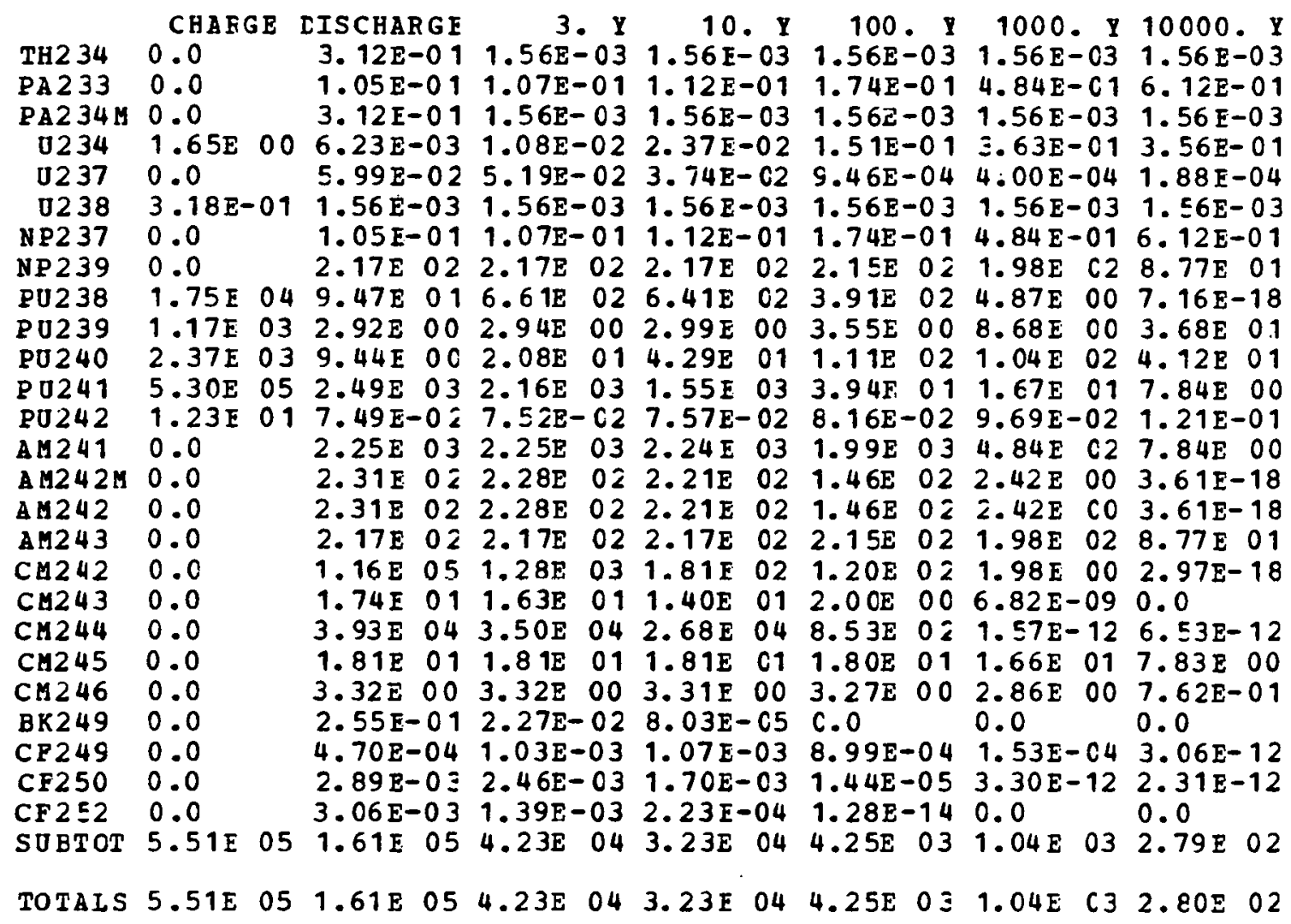


Table A.8. Curies of fission product isotopes in the high-level waste from one metric ton of plutonium-enriched BWR fuel as a function of time

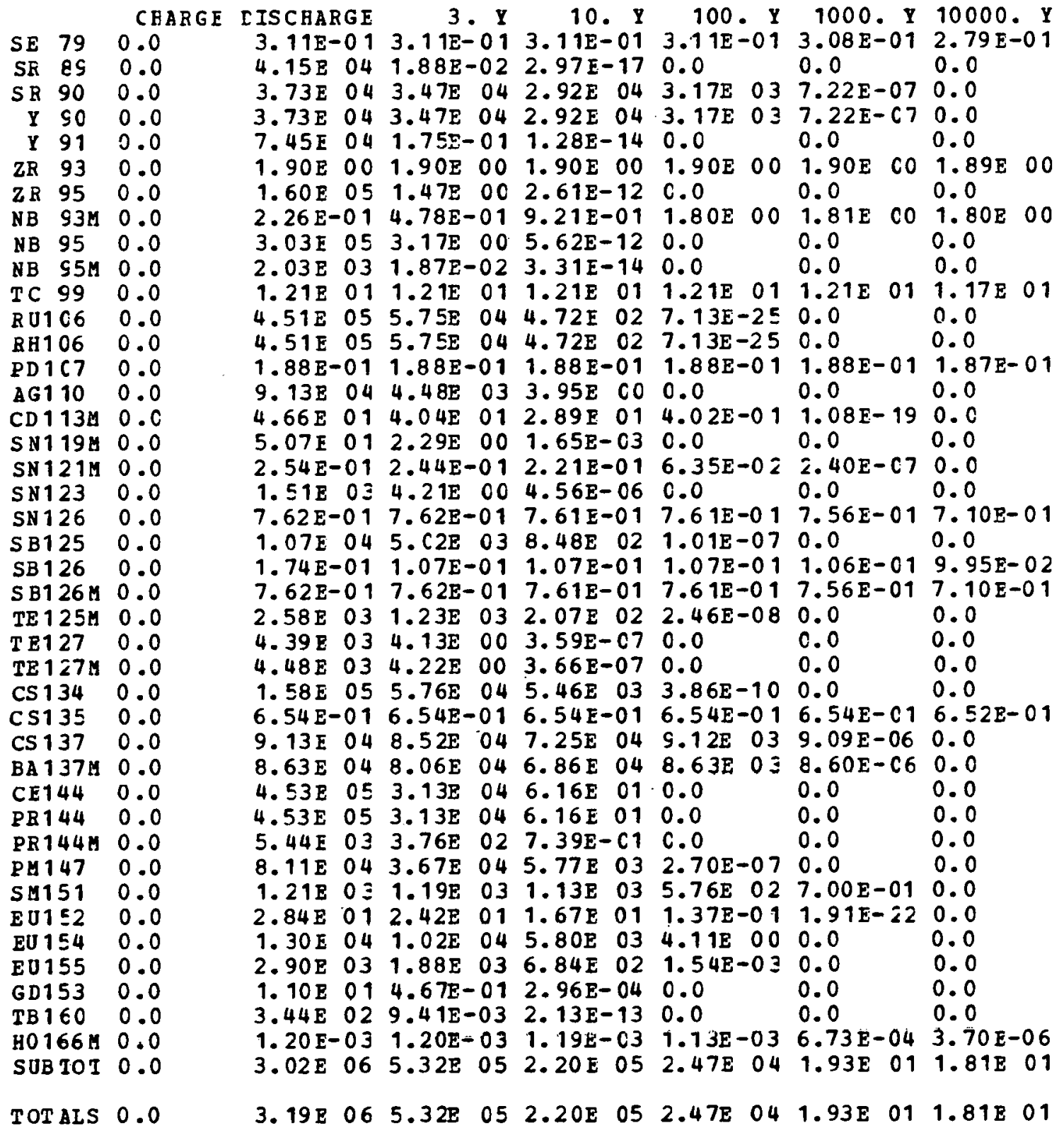


Table A.9. Curies of heavy isotopes in the high-level waste from one metric ton of blended LMFBR fuel as a function of time

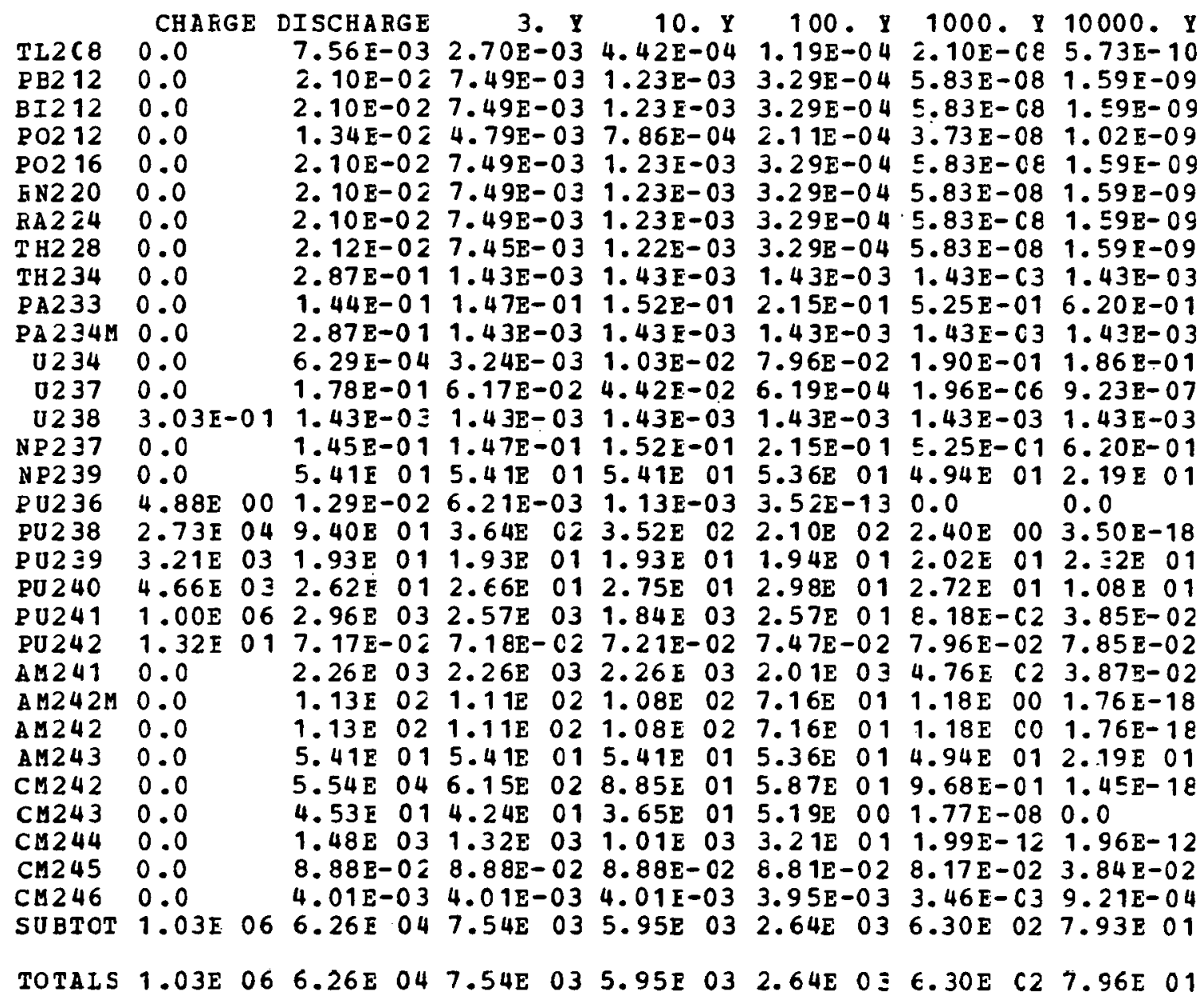


Table A.10. Curies of fission product isotopes in the high-level waste from one metric ton of blended IMFBR fuel as a function of time

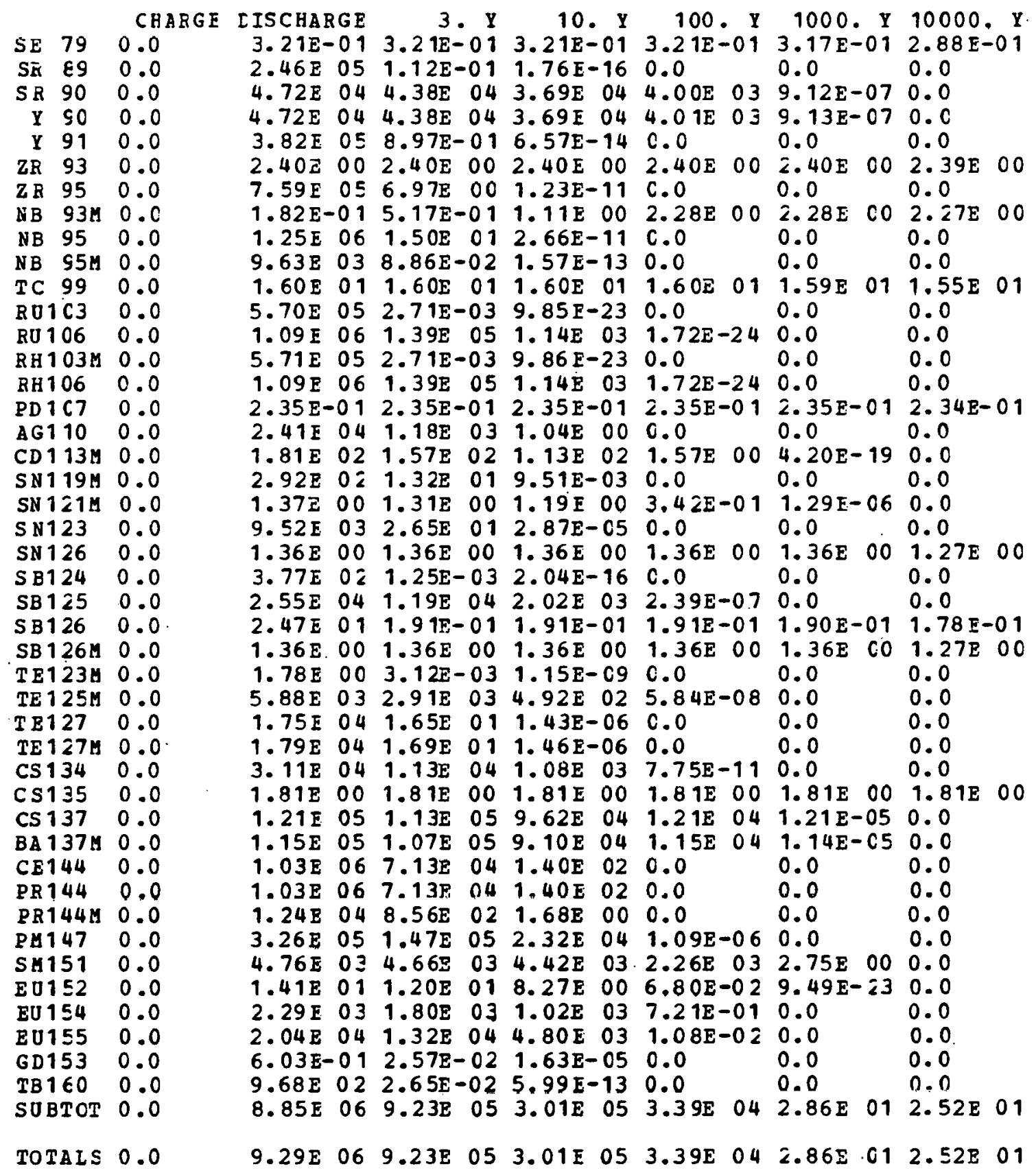


Table A.ll. Curies of heavy isotopes in the high-level waste from one metric ton of HTGR fuel as a function of time

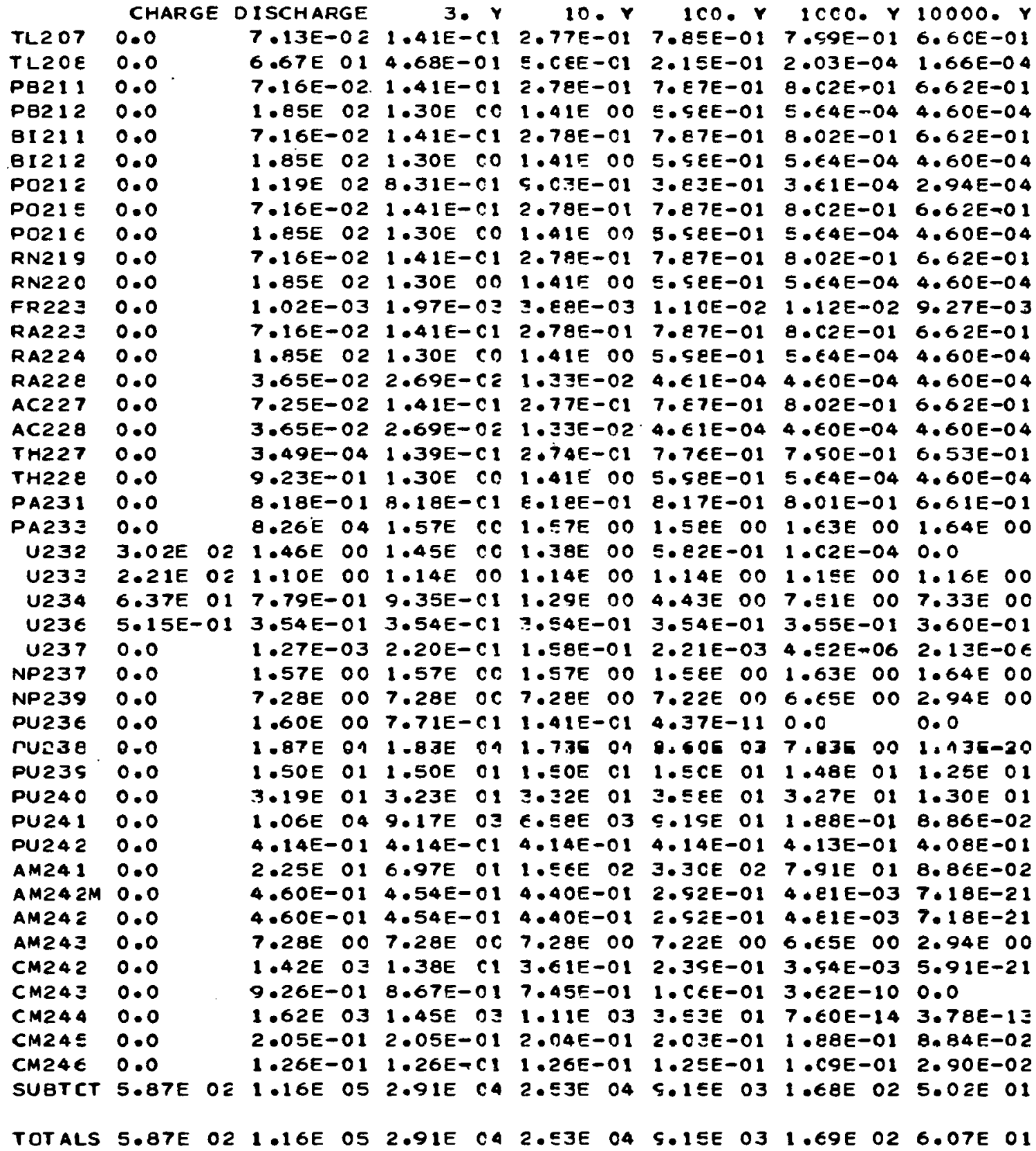


Table A.12. Curies of fission product isotopes in the high-level waste from one metric ton of HTGR fuel as a function of time

\begin{tabular}{|c|c|c|c|c|c|c|c|c|c|c|c|c|c|c|}
\hline & & CHA & I SCHAR & RGE & - & $\mathbf{Y}$ & & $\mathbf{V}$ & $1 \mathrm{CO}$ & & 1000 & $\mathbf{Y}$ & ( & \\
\hline$E$ & 9 & 0.0 & $1.79 E$ & 00 & $.79 E$ & $\mathrm{CO}$ & . T9E & 00 & $1.75 E$ & 00 & $1.77 \mathrm{E}$ & 00 & $1.61 \mathrm{E}$ & 00 \\
\hline SR & 89 & 0.0 & $.05 E$ & 05 & $.76 E-$ & C 2 & . SIE- & -17 & $0 . \mathrm{C}$ & & 0.0 & & 0.0 & \\
\hline$R$ & 90 & 0.0 & $3.06 E$ & 05 & $.84 E$ & 05 & . $39 E$ & 05 & $2.55 E$ & 04 & 5.92E & -06 & 0.0 & \\
\hline$r$ & 90 & 0.0 & $3.06 E$ & 05 & $.84 E$ & $C E$ & . $39 E$ & .05 & $2.5 S E$ & 04 & $5.92 E$ & -06 & 0.0 & \\
\hline$r$ & 91 & 0.0 & $1.57 E$ & 05 & $3.68 E-$ & .01 & $2 \cdot 69 E$ & -14 & $0 . \mathrm{C}$ & & $\mathrm{O} \cdot \mathrm{C}$ & & 0.0 & \\
\hline ZR & 93 & 0.0 & $1.03 E$ & 01 & $1.03 E$ & Cl & $1.03 E$ & 01 & 1.C.JE & 01 & $1.03 E$ & 01 & $1.02 E$ & \\
\hline$Z R$ & 95 & 0.0 & $2.32 E$ & 05 & $2.13 E$ & $\mathrm{Co}$ & 3.7RE & -12 & $0 . \mathrm{C}$ & & 0.0 & & 0.0 & \\
\hline B & $9 \geq M$ & .0 & $.40 E$ & 00 & $2.73 E$ & 00 & S. ๑8E & 00 & S. $77 E$ & 00 & 9.79E & 00 & $9.73 E$ & 00 \\
\hline IB & 95 & 0.0 & $.73 E$ & 05 & $.60 E$ & 00 & \&.15E & -12 & 0.0 & & 0.0 & & 0.0 & \\
\hline $\mathbf{B}$ & $95 M$ & 0.0 & $2.95 E$ & 03 & $2.71 \mathrm{E}-$ & 02 & 4.8OE- & -14 & 0.0 & & 0.0 & & 0.0 & \\
\hline C & 99 & 0.0 & $3 \cdot 39 E$ & 01 & $3 \cdot 39 E$ & C 1 & $3.39 E$ & 01 & $3.38 E$ & 01 & $3.37 E$ & 01 & $3.28 E$ & \\
\hline$U$ & 106 & 0.0 & $1.17 E$ & 05 & $1.50 E$ & $\mathrm{CA}$ & $1.23 E$ & 02 & 1. $\varepsilon \in E-$ & -25 & $0 . c$ & & 0.0 & \\
\hline d1 & 106 & 0.0 & $1.17 E$ & 05 & $1.50 E$ & Ca & 1. ¿ JE & 02 & 1. $\varepsilon \in E-$ & -25 & 0.0 & & 0.0 & \\
\hline D1 & 107 & 0.0 & $4.51 E$ & -02 & $4.51 \mathrm{E}-$ & $\mathrm{C}_{2}$ & $4.51 E$ & .02 & $4.51 E-$ & -02 & $4.51 E$ & -02 & $4.51 \mathrm{E}-$ & 0 \\
\hline GI & 110 & 0.0 & $1.55 E$ & 04 & $7.62 \mathrm{E}$ & 02 & 6.7 IE & -01 & $0 . \mathrm{C}$ & & $0 . c$ & & 0.0 & \\
\hline & $113 M$ & 0.0 & $4.08 E$ & 01 & $3.54 E$ & C1 & $2.54 E$ & 01 & $3.52 E$ & 01 & $9.44 E$ & 20 & 0.0 & \\
\hline & $119 M$ & .0 & $5.97 E$ & 01 & $2.69 E$ & OC & 1. S5E & -03 & $c \cdot c$ & & 0.0 & & 0.0 & \\
\hline & $121 M$ & 0.0 & $8.98 E$ & -02 & $8.62 E-$ & .02 & 7. $\varepsilon 2 E$ & $-c 2$ & $2.24 E-$ & -02 & 8.4 SE & -08 & 0.0 & \\
\hline & 123 & 0.0 & $2.92 E$ & 03 & $8.11 E$ & 00 & E. EOE & -06 & 0.0 & & $0 . C$ & & 0.0 & \\
\hline NI & 126 & 0.0 & $2,07 E$ & 00 & $2.07 E$ & 00 & $2.07 E$ & 00 & $2.07 E$ & 00 & $2.05 E$ & 00 & $1.93 E$ & \\
\hline 31 & 125 & 0.0 & $1.94 E$ & 04 & $9.06 \mathrm{E}$ & C 3 & $1.53 E$ & 03 & $1.82 E-$ & -07 & $0 . c$ & & 0.0 & \\
\hline & 126 & 0.0 & $2.90 E$ & -01 & $2.89 E-$ & 01 & ?. 89E & .01 & $2.8 \mathrm{SE}-$ & -01 & $2.87 E$ & -01 & $2.70 E-$ & - \\
\hline & $12 \in M$ & 0.0 & $2.07 E$ & 00 & $2.07 E$ & OC & $2.07 E$ & 00 & $2 \cdot C 7 E$ & 00 & $2 \cdot \operatorname{csE}$ & 00 & $1.93 E$ & 00 \\
\hline & $123 M$ & 0.0 & $1.00 E$ & 00. & $1.76 E-$ & 03 & $6.51 E$ & -10 & 0.0 & & $0 \cdot C$ & & 0.0 & \\
\hline$E I$ & $125 M$ & 0.0 & $4.71 E$ & 03 & $2.21 E$ & CI & $3.74 E$ & 02 & $4.44 E-$ & .08 & 0.0 & & 0.0 & \\
\hline EI & 127 & .0 & $8.45 E$ & 03 & $7.95 E$ & C C & $\epsilon . \$ 1 E-$ & .07 & 0.0 & & 0.0 & & 0.0 & \\
\hline E 1 & $127 M$ & .0 & $8.62 E$ & 03 & $8.12 E$ & C C & 7. CSE & -07 & $0 . c$ & & 0.0 & & 0.0 & \\
\hline CSI & 134 & 0.0 & $6.35 E$ & 05 & $2.31 E$ & 05 & $2.20 E$ & 04 & $1.5 \varepsilon E-$ & -09 & $0 . C$ & & 0.0 & \\
\hline S 1 & 135 & 0.0 & $.00 E-$ & -01 & $9.00 E-$ & .01 & $9.00 E-$ & -61 & S. C CE- & -01 & $9.0 O E$ & -01 & $8.97 E-$ & $e^{2}$ \\
\hline 51 & 137 & 0.0 & 3.02E & 05 & $2.82 E$ & CE & $2.40 E$ & 05 & Z. C 2E & 04 & $3 . C 1 E$ & -05 & 0.0 & \\
\hline BAI & $137 M$ & 0.0 & $2.86 E$ & $0 \leq$ & $2.67 E$ & 05 & $2.27 E$ & 05 & 2.eडE & 04 & $2.85 \mathrm{E}$ & -05 & 0.0 & \\
\hline 1 & 144 & 0.0 & $1.38 E$ & 06 & $9.54 E$ & 64 & 1.ETE & 02 & $c \cdot c$ & & 0.0 & & 0.0 & \\
\hline $\boldsymbol{P}$ & 144 & 0.0 & $1.38 E$ & 06 & $9.54 E$ & $\mathrm{Ca}$ & I.ETE & 02 & $0 . c$ & & 0.0 & & 0.0 & \\
\hline $\mathbf{R}$ & $144 M$ & 0.0 & $1.66 E$ & 04 & $1.15 E$ & $0 \Xi$ & Z.25E & 00 & $C \cdot C$ & & 0.0 & & 0.0 & \\
\hline M 1 & 147 & 0.0 & $1.57 E$ & 05 & $7.10 E$ & $\mathrm{Ca}$ & $1.12 E$ & 04 & $5.24 E-$ & -07 & 0.0 & & 0.0 & \\
\hline 1 & 151 & 0.0 & $6.44 E$ & 02 & $6.30 E$ & $\mathrm{C} 2$ & $5.58 E$ & 02 & 3. CSE & 02 & $3.72 E$ & -01 & 0.0 & \\
\hline U1 & 152 & 0.0 & $2.62 E$ & 00 & $2.23 E$ & 00 & 1. SIE & 00 & 1. ¿EE- & -02 & $1.76 \mathrm{E}$ & -23 & 0.0 & \\
\hline EUI & 154 & 0.0 & $2.33 E$ & 04 & $1.83 \mathrm{E}$ & $c .4$ & $1.0 \Delta \mathrm{F}$ & na & $7 . \exists 5 \mathrm{E}$ & 00 & 0.0 & & 0.0 & \\
\hline EU 1 & 155 & 0.0 & $4.52 E$ & 03 & $2 \cdot 93 E$ & C & $1.06 E$ & 03 & $2.40 E=$ & .03 & 0.0 & & 0.0 & \\
\hline & 160 & 0.0 & $6.46 E$ & 01 & $1.77 E-$ & 63 & 4. OOE & -14 & 0.0 & & 0.0 & & 0.0 & \\
\hline E & BTCT & 0.0 & $6.06 E$ & 06 & $1.67 E$ & $c \in$ & S. $\$ 2 E$ & 05 & $1.11 \mathrm{E}$ & 05 & $6.13 E$ & 01 & $5.94 E$ & 02 \\
\hline & & & $\bullet$ & 06 & $57 E$ & & S2E & 05 & IE & 5 & $13 E$ & 1 & $.94 \mathrm{E}$ & \\
\hline
\end{tabular}


Table A.13. Thermal power as a function of time in high-level

waste generated in reprocessing one ton of spent fuel ${ }^{a}$

\begin{tabular}{|c|c|c|c|c|c|c|}
\hline \multirow{2}{*}{$\begin{array}{c}\text { Time since } b \\
\text { reprocessing } \\
\text { (years) }\end{array}$} & \multicolumn{6}{|c|}{ Thermal power (watts) } \\
\hline & PWR-U & PWR-Pu & $B W R-U$ & BWR-Pu & HTGR & LMFBR \\
\hline \multicolumn{7}{|c|}{ Fission products ${ }^{c}$} \\
\hline 0 & $1.88+4^{\mathrm{d}}$ & $2.01+4$ & $1.36+4$ & $1.44+4$ & $2.12+4$ & $3.62+4$ \\
\hline 3 & $2.87+3$ & $2.93 \div 3$ & $2.18+3$ & $2.21+3$ & $6.46+3$ & $3.03+3$ \\
\hline 10 & $9.46+2$ & $8.05+2$ & $7.65+2$ & $6.58+2$ & $3.14+3$ & $7.56+2$ \\
\hline 100 & $9.45+1$ & $7.76+=$ & $7.76+1$ & $6.46+1$ & $3.33+2$ & $8.46+1$ \\
\hline 1,000 & $1.97-2$ & $2.48-2$ & $1.67-2$ & $2.06-2$ & $4.65-2$ & $3.31-2$ \\
\hline 10,000 & $1.88-2$ & $2.36-2$ & $1.59-2$ & $1.95-2$ & $4.41-2$ & $3.12-2$ \\
\hline & \multicolumn{6}{|c|}{ Actinides ${ }^{e}$} \\
\hline 0 & $5.82+2$ & $8.23+.3$ & $4.37+2$ & $5.73+3$ & $7.51+2$ & $2.18+3$ \\
\hline 3 & $7.78+1$ & $1.88+.3$ & $6.24+1$ & $1.38+3$ & $6.61+2$ & $1.62+2$ \\
\hline 10 & $5.85+1$ & $1.42+.3$ & $4.78+1$ & $1.05+3$ & $6.19+2$ & $1.31+2$ \\
\hline 100 & $1.12+1$ & $1.43+2$ & $1.12+1$ & $1.26+2$ & $2.99+2$ & $8.12+1$ \\
\hline 1,000 & $2.66+0$ & $2.97+1$ & $2.67+0$ & $2.80+: 1$ & $5.17+0$ & $1.94+1$ \\
\hline 10,000 & $5.01-1$ & $7.85+D$ & $4.25-1$ & $6.32+0$ & $1.62+0$ & $1.92+0$ \\
\hline
\end{tabular}

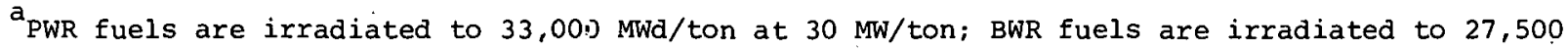
$\mathrm{MWd} /$ ton at $20.7 \mathrm{MW} /$ ton; HTGR fuels are irradiated to $94,270 \mathrm{MWd} /$ ton at $64.6 \mathrm{MW} /$ ton; LMFBR core and blankets are irradiate 3 to an average of $37,120 \mathrm{MWd} /$ ton at $64.6 \mathrm{MW} / \mathrm{ton}$.

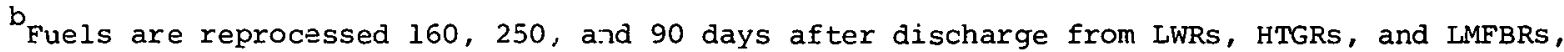
respectively.

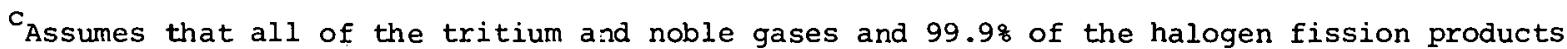
are separated from the waste during reprocessing.

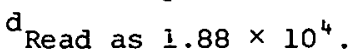

e Consists of all the actinides remaining after removal of $99.5 \%$ of the uranium and plutonium during reprocessing. 
APPENDIX B

Radioactivity and Thermal Power of the Cladding waste from One Metric Ton of Heavy Metal as a Function of Time for Five Reactor Types 
Table B.1. Curies of induced radioactivity in the cladding waste from one metric ton of uranium-enriched PWR fuel as a function of time

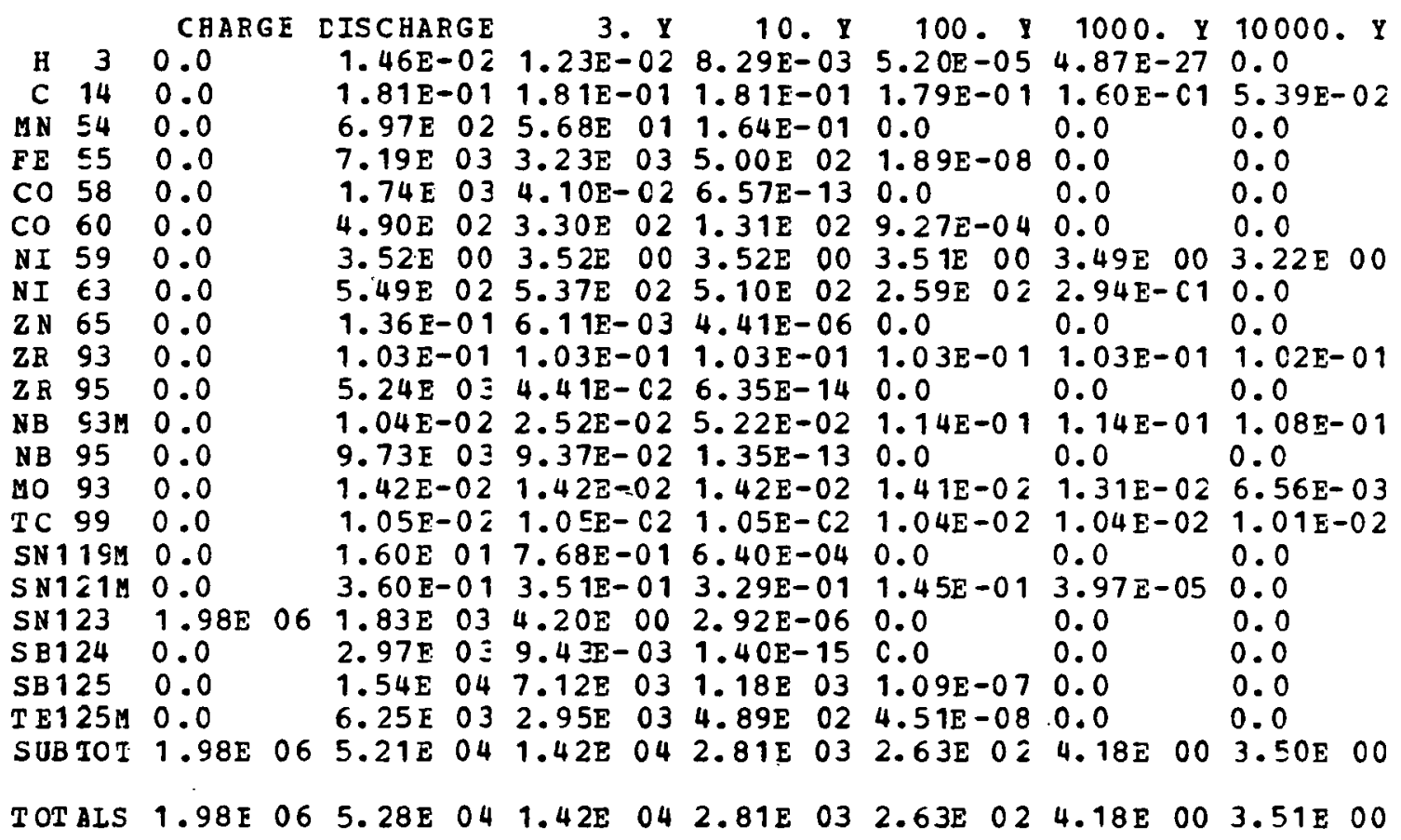


Table B.2. Curies of heavy isotopes in the cladding waste from one metric ton of uranium-enriched PWR fuel as a function of time

\begin{tabular}{|c|c|c|c|c|c|c|c|}
\hline 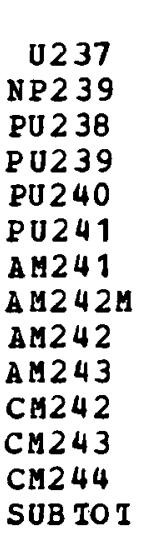 & $\begin{array}{l}\text { CHARGE } \\
0.0 \\
0.0 \\
0.0 \\
0.0 \\
0.0 \\
0.0 \\
0.0 \\
0.0 \\
0.0 \\
0.0 \\
0.0 \\
0.0 \\
0.0 \\
0.0\end{array}$ & $\begin{array}{r}\text { CISCHARGE } \\
1.53 \mathrm{E}-03 \\
8.67 \mathrm{E}-03 \\
2.50 \mathrm{E}-01 \\
1.62 \mathrm{E}-01 \\
2.41 \mathrm{E}-01 \\
6.18 \mathrm{E}-01 \\
1.19 \mathrm{E}-01 \\
3.62 \mathrm{E}-03 \\
3.62 \mathrm{E}-03 \\
8.67 \mathrm{E}-03 \\
6.84 \mathrm{E} 00 \\
1.46 \mathrm{E}-03 \\
9.86 \mathrm{E}-01 \\
7.05 \mathrm{E} 01\end{array}$ & $\begin{array}{r}3 . Y \\
1.29 E-03 \\
8.67 E-03 \\
2.77 E-01 \\
1.62 E-01 \\
2.41 E-01 \\
5.36 E 01 \\
3.95 E-01 \\
3.57 E-03 \\
3.57 E-03 \\
8.67 E-03 \\
6.77 E-02 \\
1.37 E-03 \\
8.79 E-01 \\
5.57 E 01\end{array}$ & $\begin{array}{l}10.1 \\
9.24 E-04 \\
8.66 E-03 \\
2.63 E-01 \\
1.62 E-01 \\
2.42 E-01 \\
3.85 E 01 \\
8.99 E-01 \\
3.46 E-03 \\
3.46 E-03 \\
8.66 E-03 \\
2.84 E-03 \\
1.18 E-03 \\
6.73 E-01 \\
4.07 E 01\end{array}$ & $\begin{array}{l}100 . Y \\
1.29 E-05 \\
8.59 E-03 \\
1.32 E-01 \\
1.62 E-01 \\
2.41 E-01 \\
5.36 E-01 \\
1.92 E 00 \\
2.30 E-03 \\
2.30 E-03 \\
8.59 E-03 \\
1.88 E-03 \\
1.68 E-04 \\
2.14 E-02 \\
3.04 E 00\end{array}$ & $\begin{array}{l}1000 . Y \\
4.94 E-10 \\
7.92 E-03 \\
1.90 E-04 \\
1.58 E-01 \\
2.20 E-01 \\
2.06 E-05 \\
4.59 E-01 \\
3.79 E-05 \\
3.79 E-05 \\
7.92 E-03 \\
3.11 E-05 \\
5.72 E-13 \\
2.35 E-17 \\
8.53 E-01\end{array}$ & $\begin{array}{l}10000 . \quad \mathrm{P} \\
2.32 \mathrm{E}-10 \\
3.50 \mathrm{E}-03 \\
1.12 \mathrm{E}-22 \\
1.23 \mathrm{E}-01 \\
8.74 \mathrm{E}-02 \\
9.68 \mathrm{E}-06 \\
9.94 \mathrm{E}-06 \\
5.66 \mathrm{E}-23 \\
5.66 \mathrm{E}-23 \\
3.50 \mathrm{E}-03 \\
4.65 \mathrm{E}-23 \\
0.0 \\
5.85 \mathrm{E}-18 \\
2.18 \mathrm{E}-01\end{array}$ \\
\hline & & $.05 \mathrm{E} 01$ & & & $4 E$ & $57 \mathrm{E}-0$ & $.22 \mathrm{E}-0$ \\
\hline
\end{tabular}


Table B.3. Curies of fission product isotopes in the cladding waste from one metric ton of uranium-enriched PWR fuel as a function of time

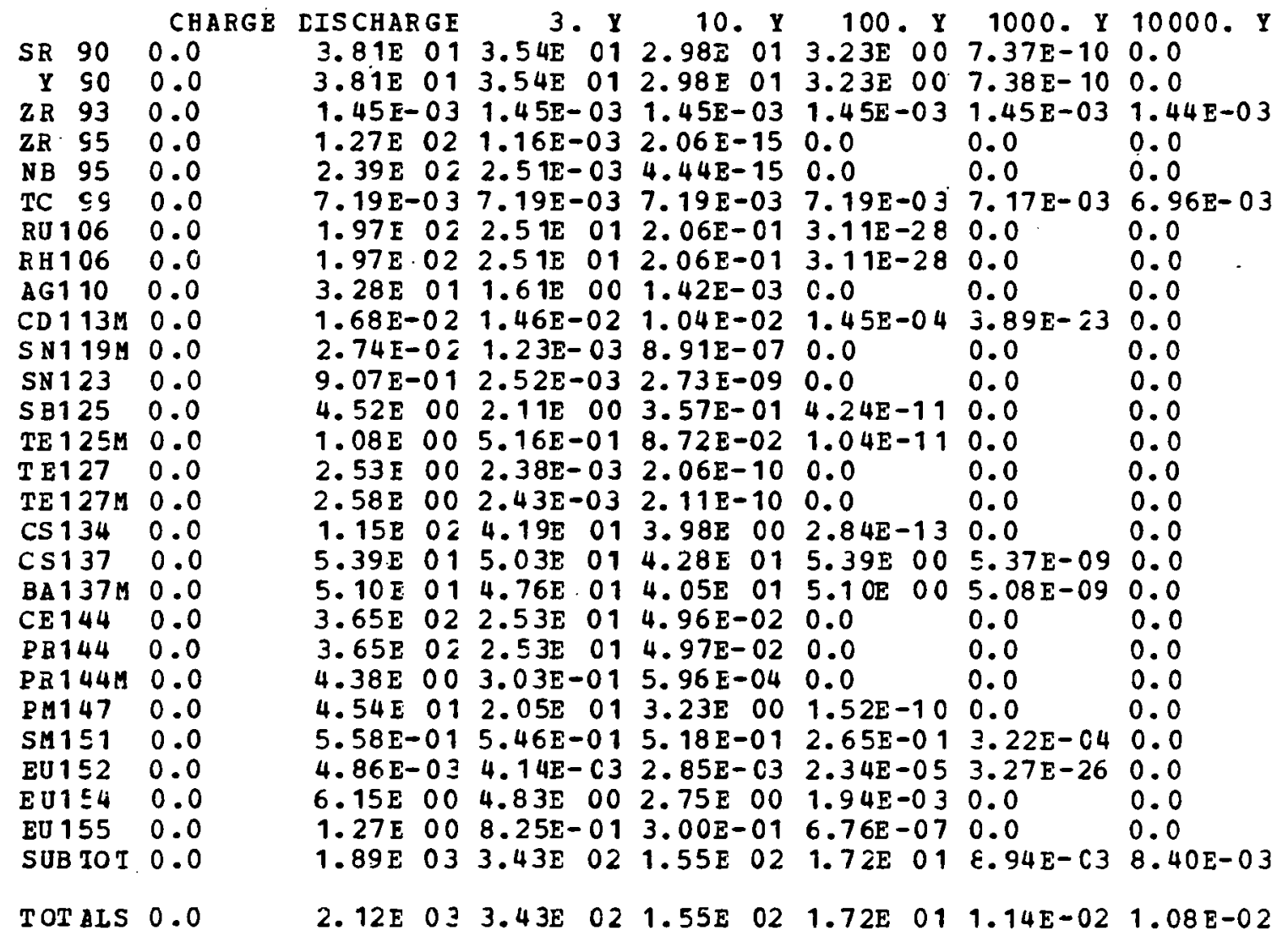


Table B.4. Curies of induced radioactivity in the cladding waste from one metric ton of plutonium-enriched PWR fuel as a function of time

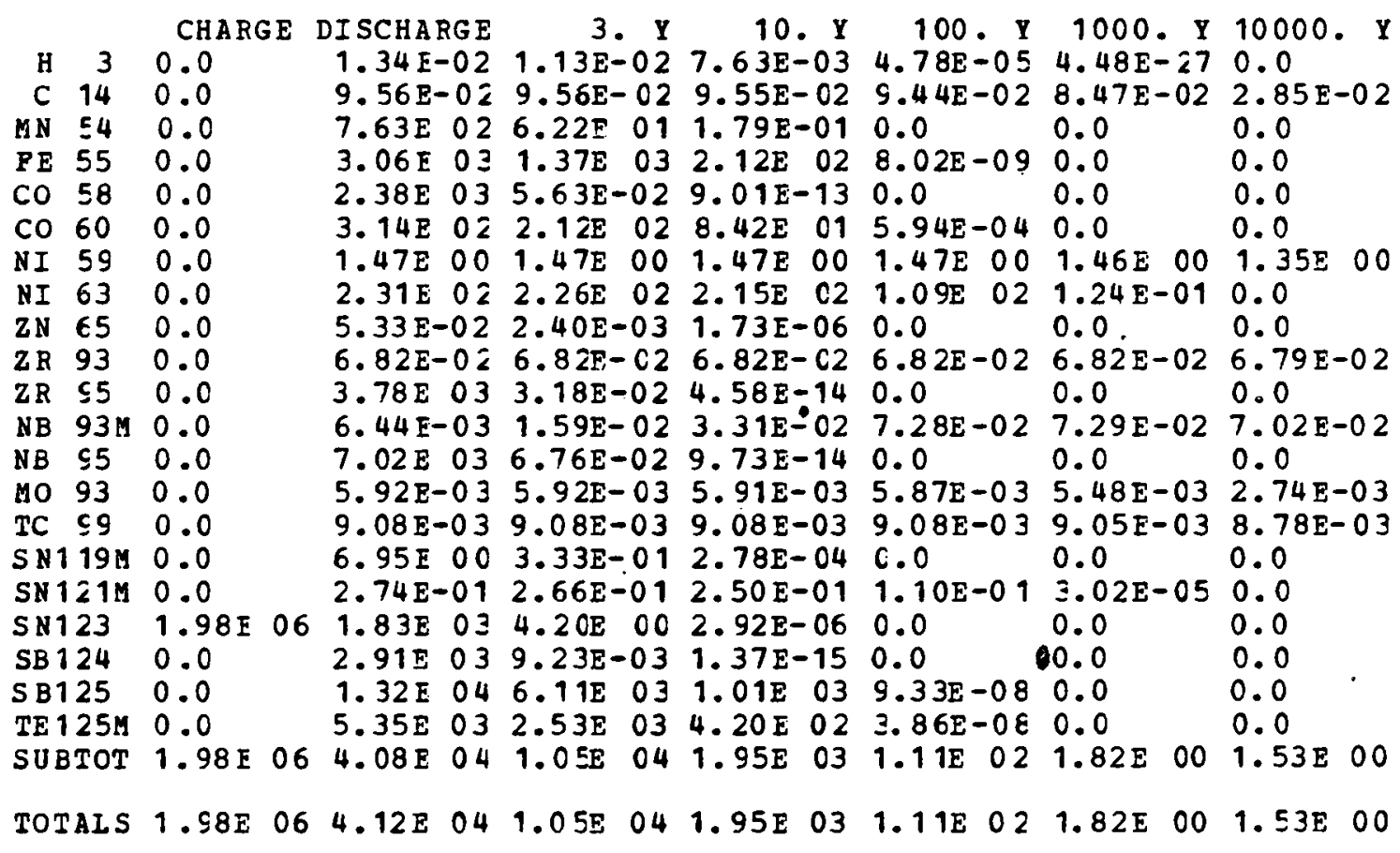


Table B.5. Curies of heavy isotopes in the cladding waste from one metric ton of plutonium-enriched PWR fuel as a function of time

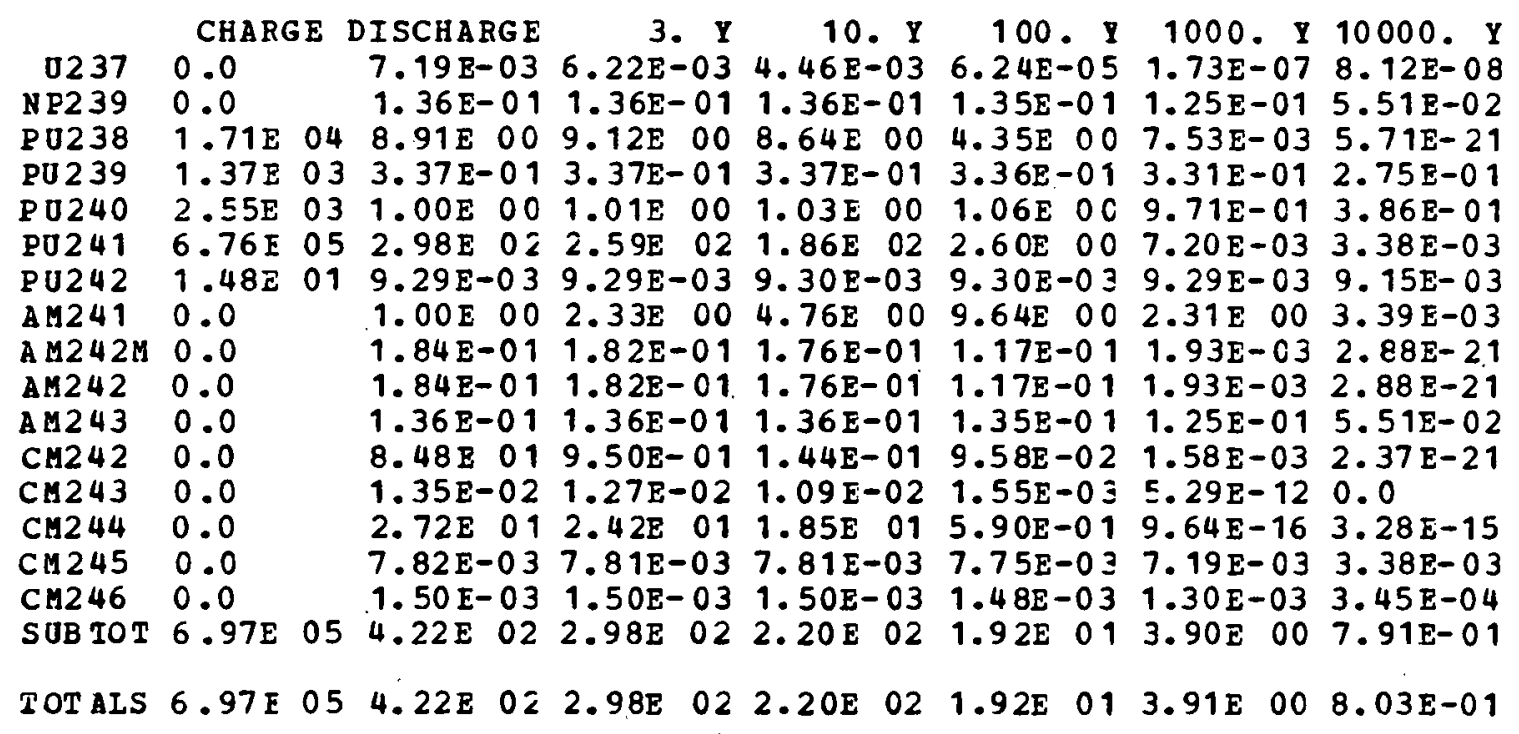


Table B.6. Curies of fission product isotopes in the cladding waste from one metric ton of plutonium-enriched PWR fuel as a function of time

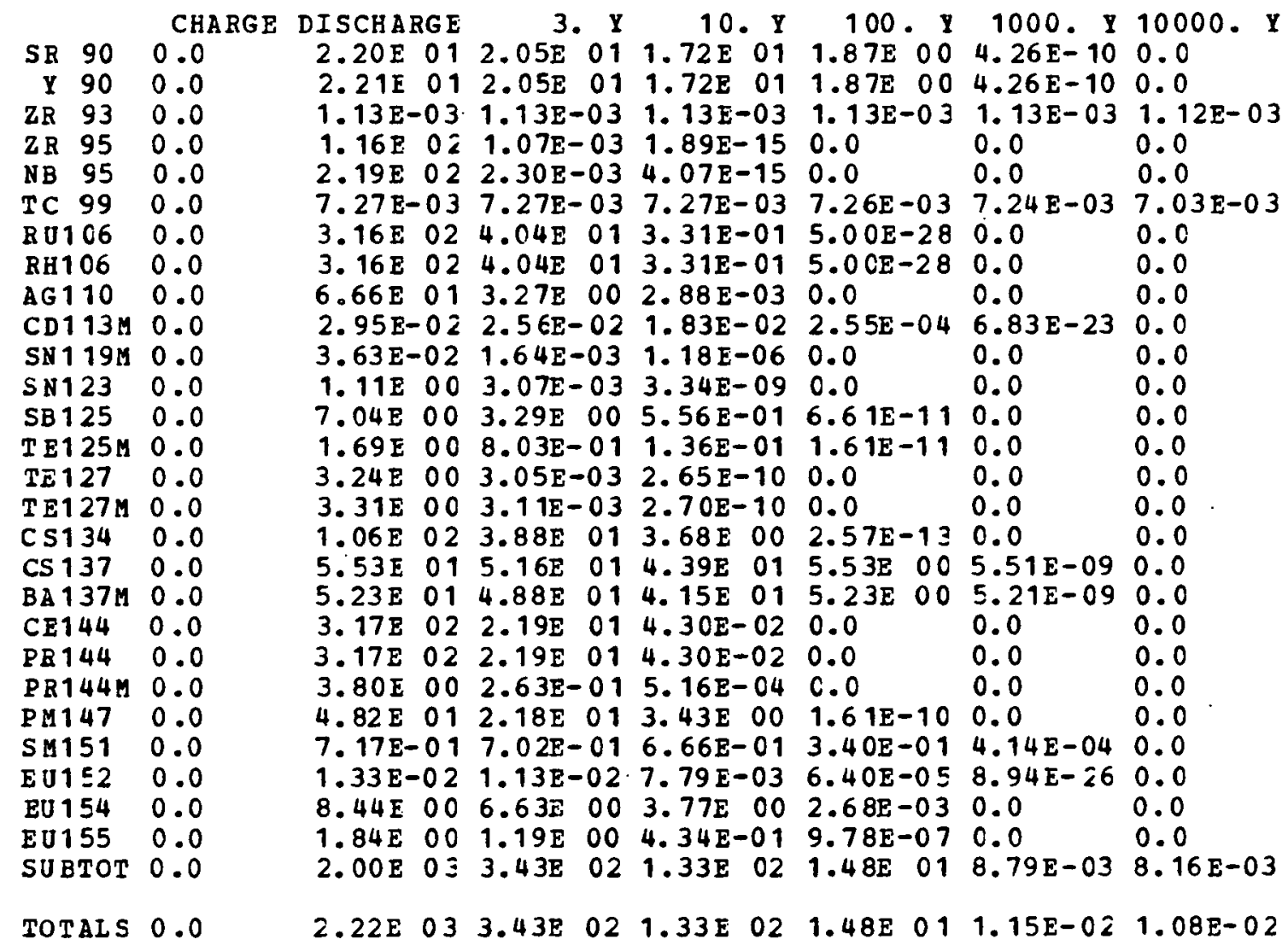


Table B.7. Curies of induced radioactivity in the cladding waste from one metric ton of uranium-enriched BWR fuel as a function of time

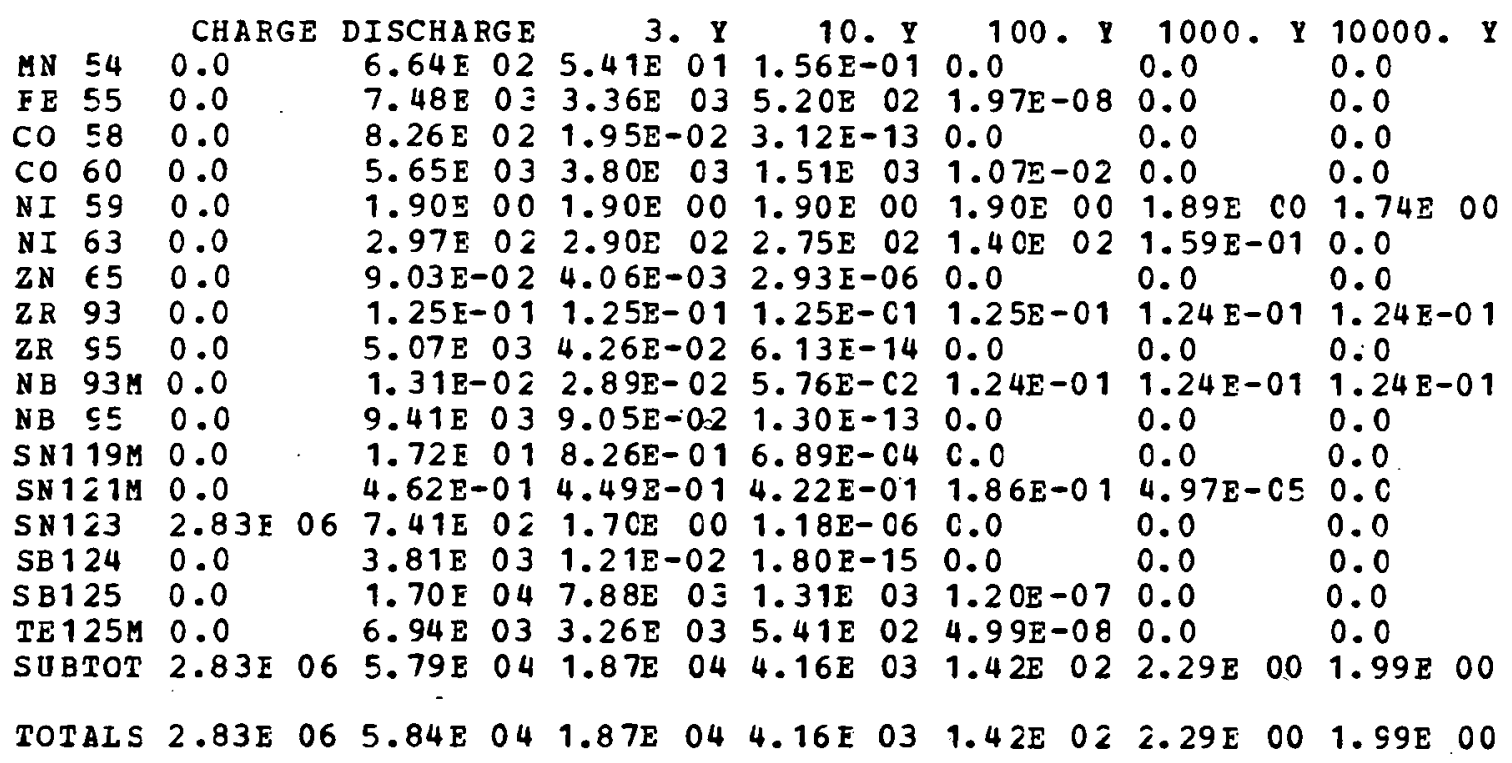


Table B.8. Curies of heavy isotopes in the cladding waste from one metric ton of uranium-enriched BWR fuel as a function of time

\begin{tabular}{|c|c|c|c|c|c|c|c|}
\hline $\begin{array}{r}\text { U2 } 37 \\
\text { NP239 } \\
\text { PU238 } \\
\text { PU239 } \\
\text { PU } 240 \\
\text { PU2 } 41 \\
\text { AM2 } 41 \\
\text { AM2 } 42 M \\
\text { AM242 } \\
\text { A } 243 \\
\text { CM2 } 42 \\
\text { CM2 } 44 \\
\text { SUBTOT }\end{array}$ & $\begin{array}{l}\text { CHARGE } \\
0.0 \\
0.0 \\
0.0 \\
0.0 \\
0.0 \\
0.0 \\
0.0 \\
0.0 \\
0.0 \\
0.0 \\
0.0 \\
0.0 \\
0.0\end{array}$ & $\begin{array}{r}\text { IISCHARGE } \\
1.32 \mathrm{E}-0 \mathrm{Z} \\
6.81 \mathrm{E}-03 \\
1.00 \mathrm{E} 0 \mathrm{C} \\
1.52 \mathrm{E}-01 \\
2.33 \mathrm{E}-01 \\
5.38 \mathrm{E} 01 \\
1.30 \mathrm{E}-01 \\
5.95 \mathrm{E}-03 \\
5.95 \mathrm{E}-0 \mathrm{3} \\
6.81 \mathrm{E}-03 \\
5.08 \mathrm{E} 00 \\
7.54 \mathrm{E}-01 \\
6.11 \mathrm{E} 01\end{array}$ & $\begin{array}{r}3 . \mathrm{Y} \\
1.12 \mathrm{E}-03 \\
6.81 \mathrm{E}-03 \\
1.00 \mathrm{O} 00 \\
1.52 \mathrm{E}-01 \\
2.33 \mathrm{E}-01 \\
4.66 \mathrm{E} 01 \\
3.70 \mathrm{E}-01 \\
5.87 \mathrm{E}-03 \\
5.87 \mathrm{E}-03 \\
6.81 \mathrm{E}-03 \\
5.28 \mathrm{E}-02\end{array}$ & $\begin{array}{r}10 . \mathrm{Y} \\
8.04 \mathrm{E}-04 \\
6.81 \mathrm{E}-03 \\
9.49 \mathrm{E}-01 \\
1.52 \mathrm{E}-01 \\
2.34 \mathrm{E}-01 \\
3.34 \mathrm{E} 01 \\
8.08 \mathrm{E}-01 \\
5.68 \mathrm{E}-03 \\
5.68 \mathrm{E}-03 \\
6.81 \mathrm{E}-03 \\
4.66 \mathrm{E}-03 \\
5.14 \mathrm{E}-01 \\
3.61 \mathrm{E} 01\end{array}$ & $\begin{array}{l}100 . Y \\
1.12 \mathrm{E}-05 \\
6.75 \mathrm{E}-03 \\
4.73 \mathrm{E}-01 \\
1.52 \mathrm{E}-01 \\
2.33 \mathrm{E}-01 \\
4.67 \mathrm{E}-01 \\
1.69 \mathrm{E} 00 \\
3.77 \mathrm{E}-03 \\
3.77 \mathrm{E}-03 \\
6.75 \mathrm{E}-03 \\
3.09 \mathrm{E}-03 \\
1.64 \mathrm{E}-02 \\
3.06 \mathrm{E} 00\end{array}$ & $\begin{array}{l}1000 . Y \\
1.09 E-08 \\
6.22 E-C 3 \\
5.45 E-04 \\
1.48 E-01 \\
2.12 E-01 \\
4.56 E-C 4 \\
4.05 E-01 \\
6.22 E-C 5 \\
6.22 E-05 \\
6.22 E-03 \\
5.10 E-05 \\
2.87 E-17 \\
7.79 E-01\end{array}$ & $\begin{array}{l}10000 . Y \\
5.14 E-09 \\
2.75 E-03 \\
1.84 E-22 \\
1.16 E-01 \\
8.43 E-02 \\
2.14 E-04 \\
2.15 E-04 \\
9.29 E-23 \\
9.29 E-23 \\
2.75 E-03 \\
7.64 E-23 \\
1.10 E-16 \\
2.06 E-01\end{array}$ \\
\hline & & & & & & & \\
\hline
\end{tabular}


Table B.9. Curies of fission product isotopes in the cladding waste from one metric ton of uranium-enriched BWR fuel as a function of time

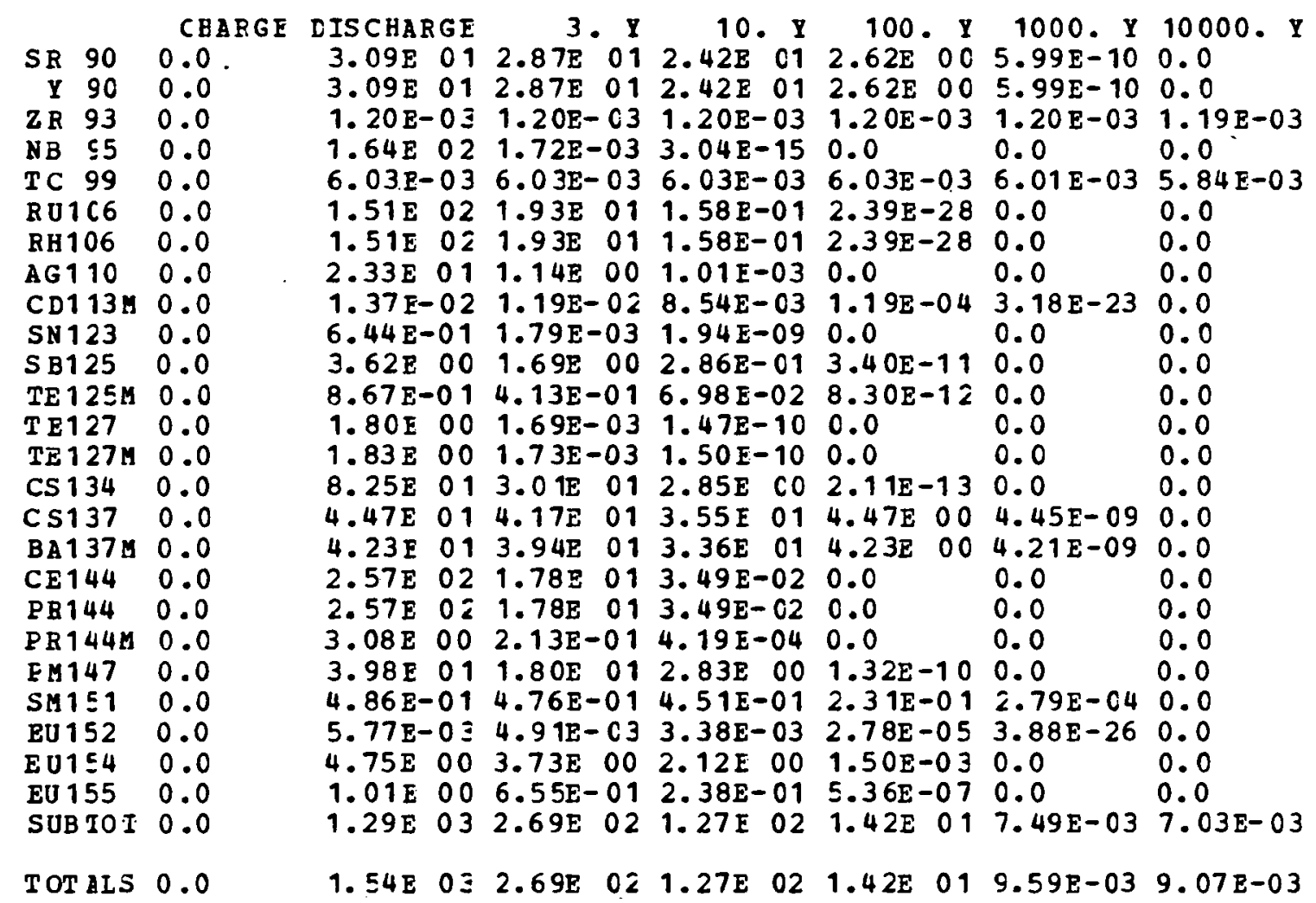


Table B.10. Curies of induced radioactivity in the cladding waste from one metric ton of plutonium-enriched BWR fuel as a function of time

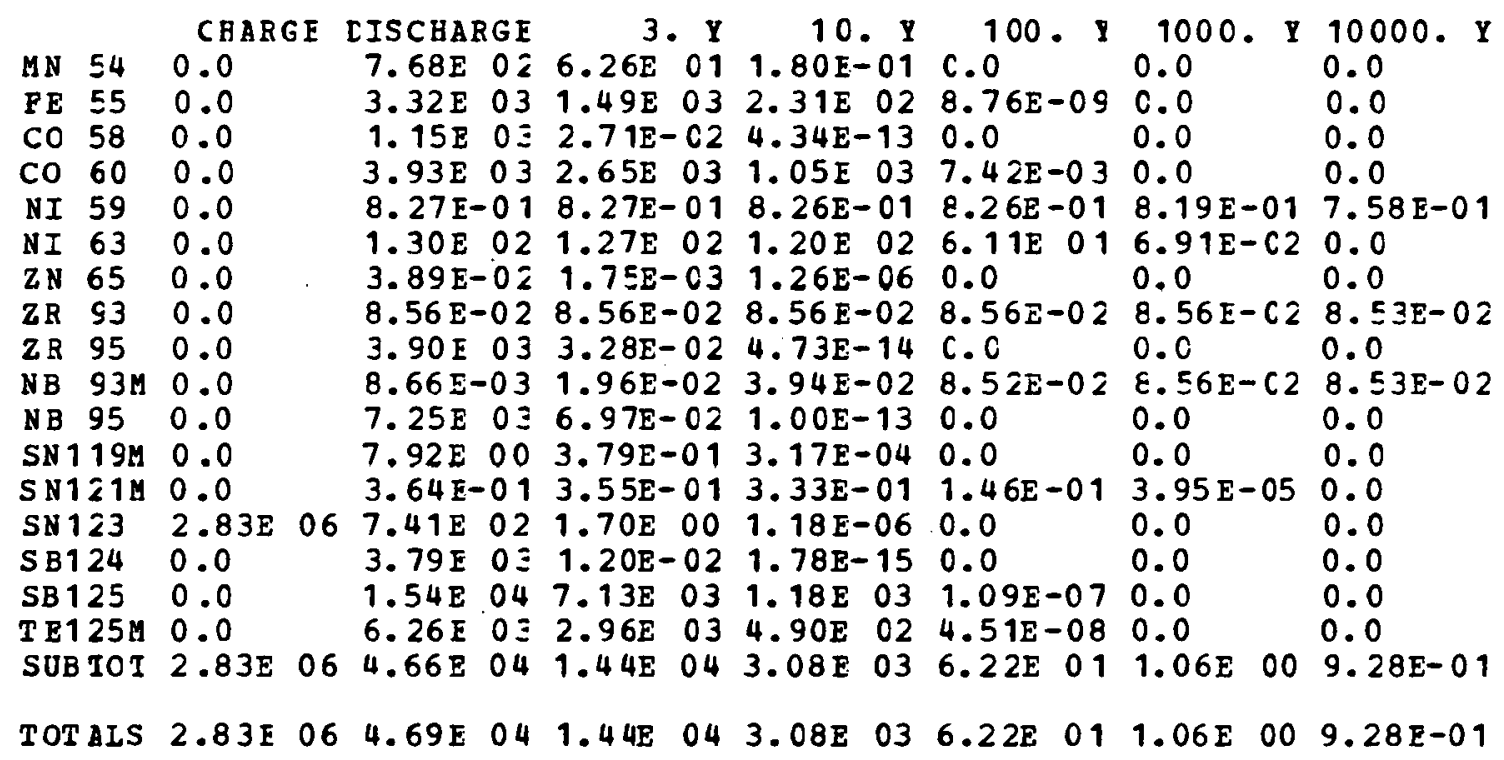


Table B.1l. Curies of heavy isotopes in the cladding waste from one metric ton of plutonium-enriched BWR fuel as a function of time

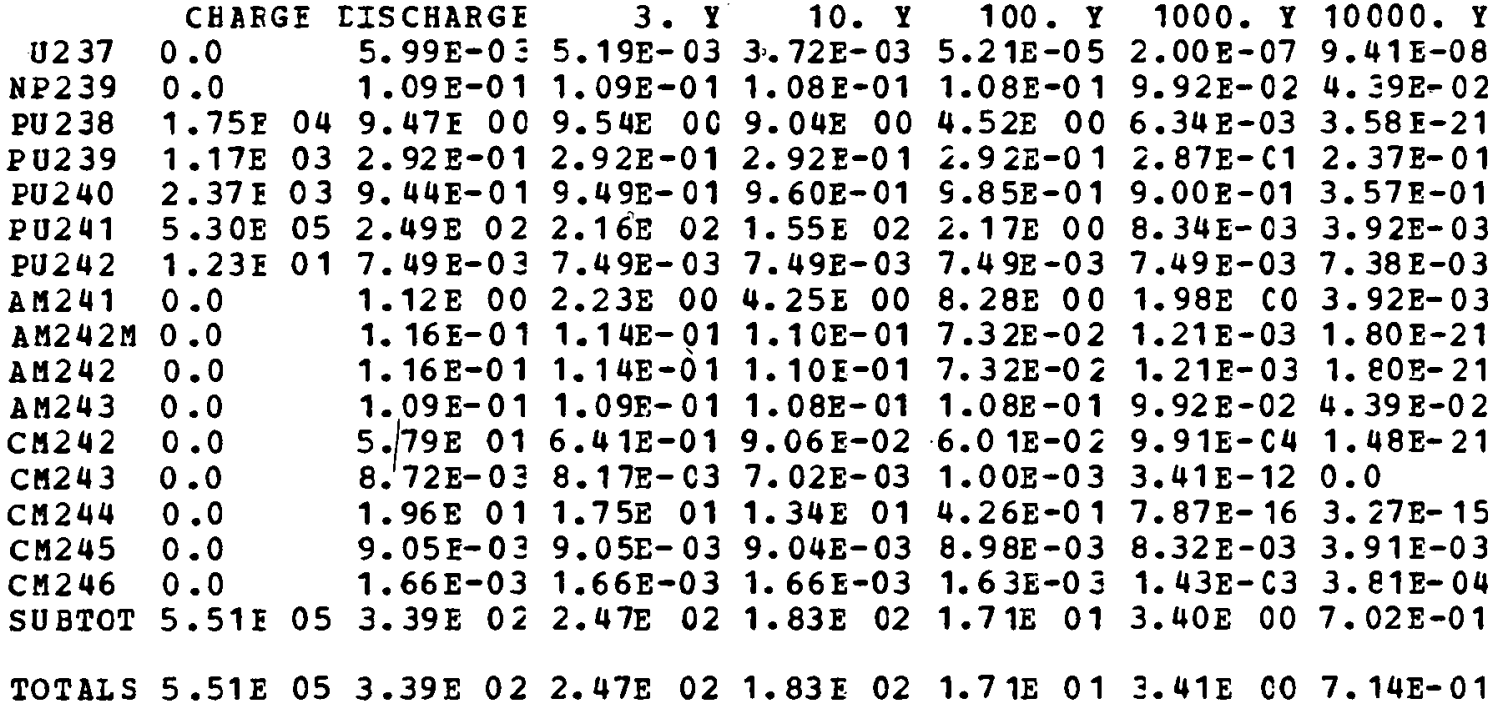


Table B.12. Curies of fission product isotopes in the cladding waste from one metric ton of plutonium-enriched BWR fuel as a function of time

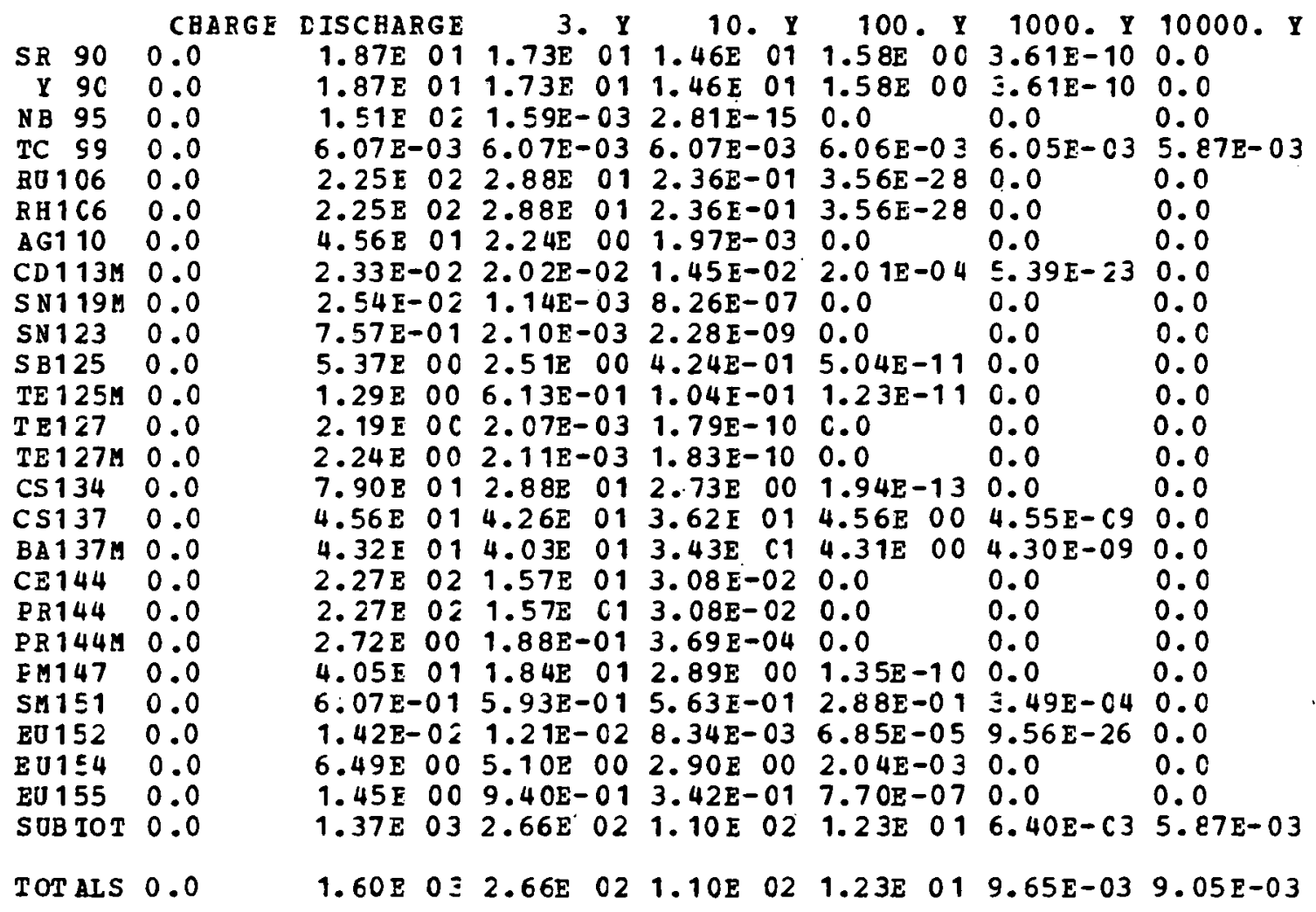


Table B.13. Curies of induced radioactivity in the cladding waste from one metric ton of blended LMFBR fuel as a function of time

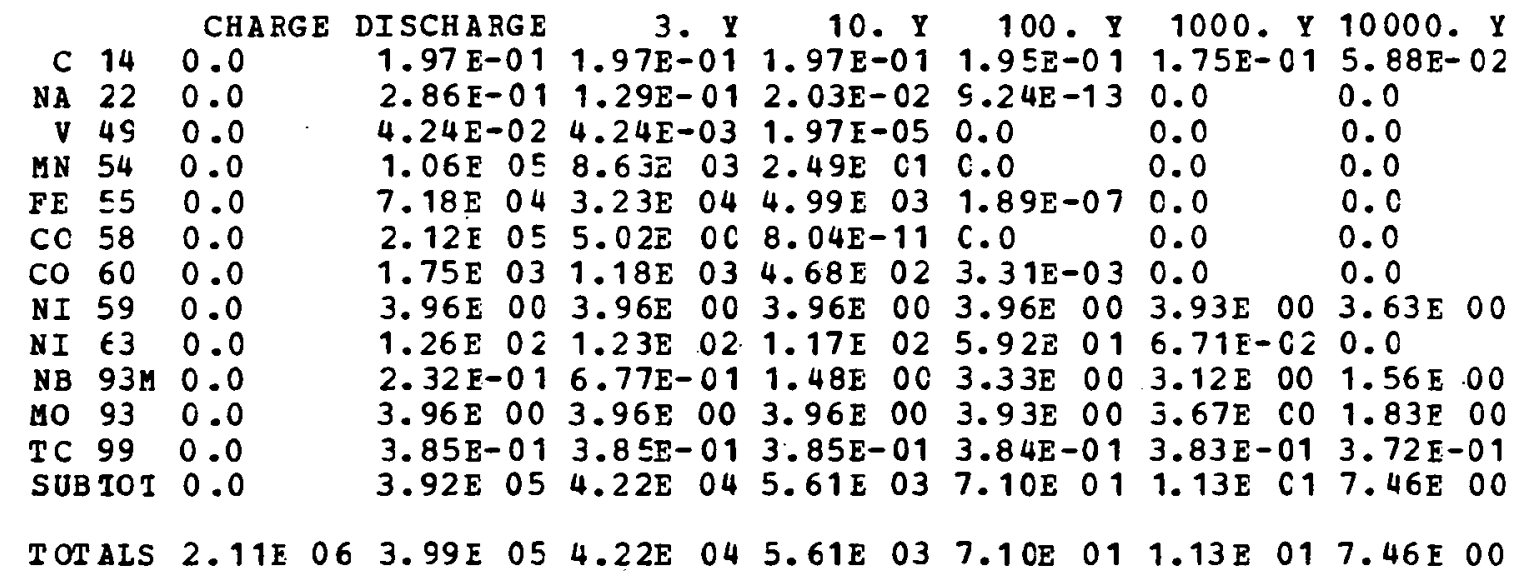


Table B.14. Curies of heavy isotopes in the cladding waste from one metric ton of blended LMFBR fuel as a function of time

\begin{tabular}{|c|c|c|c|c|c|c|c|c|c|c|c|}
\hline $\begin{array}{l}0 \\
11 \\
2 \\
1 \\
24 \\
24 \\
3 \\
2 \\
3\end{array}$ & $\begin{array}{l}\text { CHARGI } \\
0.0 \\
0.0 \\
2.73 \mathrm{E} \\
3.21 \mathrm{E} \\
4.66 \mathrm{E} \\
1.00 \mathrm{E} \\
1.32 \mathrm{E} \\
0.0 \\
0.0 \\
0.0 \\
0.0 \\
0.0 \\
0.0 \\
0.0 \\
1.03 \mathrm{E} \\
0\end{array}$ & $\begin{array}{l}04 \\
03 \\
03 \\
06 \\
01\end{array}$ & $\begin{array}{r}\text { DISCHARGE } \\
1.78 \mathrm{E}-02 \\
2.71 \mathrm{E}-02 \\
9.40 \mathrm{E} 00 \\
1.93 \mathrm{E} 00 \\
2.62 \mathrm{E} 00 \\
2.96 \mathrm{E} 02 \\
7.17 \mathrm{E}-03 \\
1.13 \mathrm{E} 00 \\
5.64 \mathrm{E}-02 \\
5.64 \mathrm{E}-02\end{array}$ & $\begin{array}{l}6.17 \mathrm{E}- \\
2.71 \mathrm{E}- \\
9.32 \mathrm{E} \\
1.93 \mathrm{E} \\
2.62 \mathrm{E} \\
2.57 \mathrm{E} \\
7.17 \mathrm{E}- \\
2.44 \mathrm{E}\end{array}$ & $\begin{array}{r}Y \\
03 \\
03 \\
-02 \\
0 C \\
00 \\
00 \\
02\end{array}$ & $\begin{array}{l}4.42 \mathrm{E}- \\
2.70 \mathrm{E}- \\
8.83 \mathrm{E} \\
1.93 \mathrm{E} \\
2.62 \mathrm{E} \\
1.84 \mathrm{E} \\
7.17 \mathrm{E}- \\
4.85 \mathrm{E} \\
5.39 \mathrm{E}- \\
5.39 \mathrm{E}-\end{array}$ & $\begin{array}{r}Y \\
-03 \\
-02 \\
00 \\
00 \\
00 \\
02 \\
-03 \\
00 \\
-02 \\
-02\end{array}$ & $\begin{array}{r}100 \\
6.17 \mathrm{E} \\
2.68 \mathrm{E}- \\
4.4 \mathrm{OE} \\
1.92 \mathrm{E} \\
2.6 \mathrm{CE} \\
2.57 \mathrm{E} \\
7.17 \mathrm{E}- \\
9.67 \mathrm{E}\end{array}$ & $\begin{array}{r}Y \\
-05 \\
-02 \\
00 \\
00 \\
00 \\
0 C \\
-03 \\
00 \\
-02 \\
-02 \\
-02\end{array}$ & $\begin{array}{l}1000 . Y \\
9.82 E-10 \\
2.47 E-C 2 \\
5.09 E-03 \\
1.87 E 00 \\
2.37 E 00 \\
4.09 E-C 5 \\
7.16 E-03 \\
2.31 E 00 \\
5.90 E-04 \\
5.90 E-C 4 \\
2.47 E-02 \\
4.84 E-04 \\
8.85 E-12 \\
1.95 E-13 \\
6.61 E 00\end{array}$ & $\begin{array}{l}200 . Y \\
2 E-10 \\
9 E-02 \\
5 E-21 \\
45 E-00 \\
1 E-01 \\
2 E-05 \\
5 E-03 \\
5 E-05 \\
1 E-22 \\
1 E-22 \\
9 E-02 \\
5 E-22 \\
5 E-13 \\
2 E 00\end{array}$ \\
\hline & & & & & & & & & & $2 E$ & $A_{\mathrm{E}}$ \\
\hline
\end{tabular}


Table B.15. Curies of fission product isotopes in the cladding waste from one metric ton of blended LMFBR fuel as a function of time

\begin{tabular}{|c|c|c|c|c|c|c|c|c|c|c|c|c|}
\hline & & $\mathrm{CH}$ & $\mathrm{HAI}$ & $G E$ & & & 0. & $\mathbf{Y}$ & 100 & & $1000 . y$ & 00. \\
\hline & 30 & 0.0 & 2. $36 \mathrm{E}$ & & $2.19 \mathrm{E}$ & 01 & $1.84 \mathrm{E}$ & 01 & $2.00 \mathrm{E}$ & 0 & 4.57 & \\
\hline $\mathbf{Y}$ & $\subseteq C$ & 0.0 & $2.36 \mathrm{E}$ & 01 & $2.19 \mathrm{E}$ & 01 & $1.84 E$ & 01 & $2.00 \mathrm{E}$ & 00 & $4.57 E-10$ & 0.0 \\
\hline $\mathbf{R}$ & 93 & 0.0 & $20 \mathrm{E}$ & & $1.20 \mathrm{E}-$ & -03 & $20 \mathrm{E}-$ & -03 & $1.2 \mathrm{OE}$ & -03 & $1.20 E-03$ & $1.19 \mathrm{E}-0$ \\
\hline$R$ & S5 & 0.0 & $3.79 E$ & 02 & $3.49 \mathrm{E}-$ & -03 & $6.17 \mathrm{E}-$ & -15 & 0.0 & & 0.0 & 0.0 \\
\hline N B & 95 & 0.0 & $6.25 \mathrm{E}$ & $0 \bar{z}$ & 7. & $0 \Xi$ & $1.33 \mathrm{E}-$ & 14 & C. 0 & & 0.0 & 0.0 \\
\hline & S9 & 0.0 & $7.99 \mathrm{E}$ & -03 & & & 7. & -03 & $7.99 E$ & & $7.97 \varepsilon-03$ & $7.73 \mathrm{E}-03$ \\
\hline 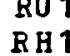 & $\begin{array}{l}106 \\
106\end{array}$ & $\begin{array}{l}0.0 \\
0.0\end{array}$ & $\begin{array}{l}43 E \\
43 E\end{array}$ & 02 & $\begin{array}{l}6 \\
6\end{array}$ & 0 & 5 & & $\begin{array}{l}8 \\
8\end{array}$ & $\begin{array}{l}-28 \\
-28\end{array}$ & & 0.0 \\
\hline & 110 & 0.0 & $1.20 E$ & 01 & $5.90 \mathrm{E}-$ & -01 & $5.21 \mathrm{E}-$ & -04 & 0.0 & & 0.0 & 0 . \\
\hline & $113 M$ & 0.0 & $9.06 \mathrm{E}$ & -02 & 7. & -02 & $5.64 \mathrm{E}-$ & -02 & $7.83 \mathrm{E}$ & -04 & $2.10 E-22$ & 0 . \\
\hline & $119 \mathrm{M}$ & 0.0 & $1.46 \mathrm{E}$ & -01 & 6. & -03 & $4.75 E-$ & -06 & 0.0 & & 0.0 & 0 \\
\hline & 123 & 0.0 & $4.76 \mathrm{E}$ & 00 & 1. & -02 & $1.44 \mathrm{E}-$ & -08 & 0.0 & & 0.0 & 0. \\
\hline & 125 & 0.0 & $1.28 \mathrm{E}$ & 01 & 5. & $O C$ & $1.01 \mathrm{E}$ & 0.0 & $1.2 \mathrm{OE}$ & -10 & 0.0 & \\
\hline & $125 M$ & 0.0 & $4 E$ & 00 & 1.2 & 00 & & -01 & $2.92 E$ & -11 & 0.0 & \\
\hline & 127 & 0.0 & $8.77 \mathrm{E}$ & 00 & $8.26 \mathrm{E}-$ & -03 & $7.17 \mathrm{E}-$ & -10 & C. 0 & & 0.0 & 0 \\
\hline & 12 & 0.0 & $\begin{array}{l}95 \mathrm{E} \\
56 \mathrm{D}\end{array}$ & 00 & 8 & & - & -10 & 0.0 & & 0.5 & 0 \\
\hline & 13. & 0.0 & $\begin{array}{l}1.56 \mathrm{E} \\
6.06 \mathrm{E}\end{array}$ & 01 & $\begin{array}{l}5.61 E \\
5.65 E\end{array}$ & 01 & $\begin{array}{l}3.38 E- \\
4.81 E\end{array}$ & $\begin{array}{r}-01 \\
01\end{array}$ & $6.18 \mathrm{E}$ & $\begin{array}{r}-14 \\
00\end{array}$ & 0.0 & 0. \\
\hline & 27 & 0.0 & $5.73 \mathrm{E}$ & 01 & $5.35 \mathrm{E}$ & 01 & $4.55 \mathrm{E}$ & 01 & $5.73 \mathrm{E}$ & 00 & $5.71 \mathrm{E}-09$ & \\
\hline & 144 & 0.0 & $5.15 \mathrm{E}$ & 02 & $3.57 \mathrm{E}$ & 01 & $7.01 \mathrm{E}-$ & -02 & 0.0 & & 0.0 & 0. \\
\hline & 144 & 0.0 & $5 E$ & 02 & $7 E$ & 01 & $1 E-$ & -02 & c & & 0 & \\
\hline & & & $6.19 \mathrm{E}$ & 00 & $4.28 \mathrm{E}-$ & -0 & $1 \mathrm{E}-$ & -04 & & & 0. & \\
\hline & 147 & 0.0 & & 02 & $37 E$ & 01 & $1.16 \mathrm{E}$ & 01 & $5.42 \mathrm{E}-$ & -10 & 0.0 & \\
\hline SM & 151 & 0.0 & & 00 & $2.33 \mathrm{E}$ & 00 & $2.21 E$ & 00 & $1.13 \mathrm{E}$ & 00 & $1.37 E-C 3$ & 0 \\
\hline & 152 & 0.0 & & $-0 ?$ & 1E- & -03 & $E-$ & -03 & $3.40 \mathrm{E}-$ & -05 & $4.75 E-26$ & 0. \\
\hline & 154 & & & 00 & & -01 & & -01 & 3. $62 \mathrm{E}$ & 04 & & \\
\hline & & & & 0 & & 0 & $O E$ & 00 & $5.41 E$ & -06 & & \\
\hline & & & & ( & & 02 & $O \mathrm{E}$ & 02 & D & & $C 2$ & - \\
\hline & & & $.65 \mathrm{E}$ & $0 \Xi$ & $62 \mathrm{E}$ & 02 & $50 \mathrm{E}$ & 02 & $69 \pi$ & 0 & $1.43 F-02$ & 1.26 \\
\hline
\end{tabular}


Table B.16. Thermal power as a function of time in the fuel structural materials associated with one ton of spent fuel ${ }^{a}$

\begin{tabular}{|c|c|c|c|c|c|}
\hline $\begin{array}{l}\text { Time since } b \\
\text { reprocessing } \\
\text { (years) }\end{array}$ & PWR-U & PWR-Pu & BWR-U & $\mathrm{BWR}-\mathrm{Pu}$ & LMFBR \\
\hline & \multicolumn{5}{|c|}{ Light elements } \\
\hline 0 & $2.37+2$ & $2.05+2$ & $3.16+2$ & $2.61+2$ & $3.92+3$ \\
\hline 3 & $4.14+1$ & $3.26+1$ & $9.91+1$ & $7.53+1$ & $1.30+2$ \\
\hline 10 & $7.99+0$ & $6.09+0$ & $3.01+1$ & $2.20+1$ & $1.41+1$ \\
\hline 100 & $4.17-2^{c}$ & $1.77-2$ & $2.28-2$ & $1.01-2$ & $2.12-2$ \\
\hline 1,000 & $2.00-4$ & $1.09-4$ & $8.37-5$ & $5.12-5$ & $1.09-2$ \\
\hline \multirow[t]{2}{*}{10,000} & $1.03-4$ & $6.44-5$ & $5.80-5$ & $3.99-5$ & $5.77-3$ \\
\hline & \multicolumn{5}{|c|}{ Fission products ${ }^{d}$} \\
\hline 0 & $9.41+0$ & $1.00+1$ & $6.79+0$ & $7.19+0$ & $1.81+1$ \\
\hline 3 & $1.44+0$ & $1.46+0$ & $1.09+0$ & $1.11+0$ & $1.52+0$ \\
\hline 10 & $4.73-1$ & $4.02-1$ & $3.83-1$ & $3.29-.1$ & $3.78-1$ \\
\hline 100 & $4.73-2$ & $3.88-2$ & $3.88-2$ & $3.23-2$ & $4.23-2$ \\
\hline 1,000 & $9.87-6$ & $1.24-5$ & $8.34-6$ & $1.03-5$ & $1.65-5$ \\
\hline \multirow[t]{2}{*}{10,000} & $9.41-6$ & $1.18-5$ & $7.95-6$ & $9.78-.6$ & $1.56-5$ \\
\hline & \multicolumn{5}{|c|}{ Actinides $^{e}$} \\
\hline 0 & $3.15-1$ & $4.46+0$ & $2.66-1$ & $3.23+0$ & $1.55+0$ \\
\hline 3 & $7.09-2$ & $1.32+0$ & $8.53-2$ & $1.08+0$ & $5.79-1$ \\
\hline 10 & $7.69-2$ & $1.16+0$ & $9.03-2$ & $9.63-1$ & $6.25-1$ \\
\hline 100 & $8.23-2$ & $5.40-1$ & $8.52-2$ & $4.88-1$ & $6.12-1$ \\
\hline 1,000 & $2.75-2$ & $1.23-1$ & $2.51-2$ & $1.08-1$ & $2.11-1$ \\
\hline 10,000 & $6.78-3$ & $2.34-2$ & $6.42-3$ & $2.09-2$ & $7.54-2$ \\
\hline
\end{tabular}

${ }^{a}$ PWR fuels are irradiated to $33,000 \mathrm{MWd} /$ ton at $30 \mathrm{MW} / \mathrm{ton}$; BWR fuels are irradiated to $27,500 \mathrm{MWd} /$ ton at $20.7 \mathrm{MW} / \mathrm{ton}$; HTGR fuels are irradiated to $94,27 \dot{\mathrm{j}} \mathrm{MWd} / \mathrm{ton}$ at $64.6 \mathrm{MW} /$ ton; LMFBR core and blankets are irradiated to an average of 37,120 MWd/ton at $64.6 \mathrm{MW} /$ ton.

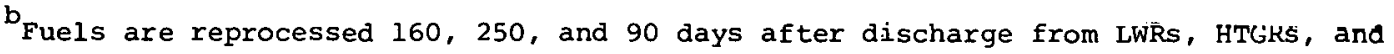
LMFBRs, respectively.

$c_{\text {Read as }} 4.17 \times 10^{-2}$

d

Assumes that 0.058 of all nonvolatile fission products are associated with the fuel structural materials.

eAssumes that $0.05 \%$ of all actinides in the spent fuel are associated with the fuel structural materials. 


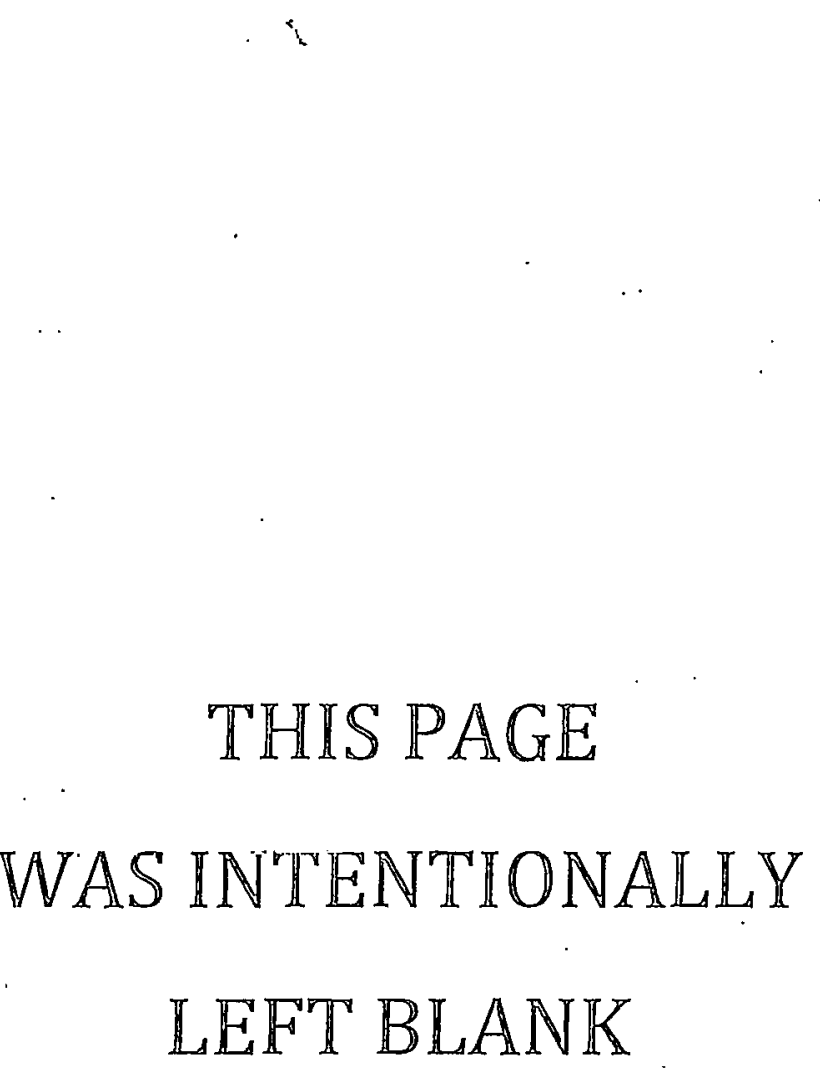


INTERNAL DISTRIBUTION

ORNL/TM-5427

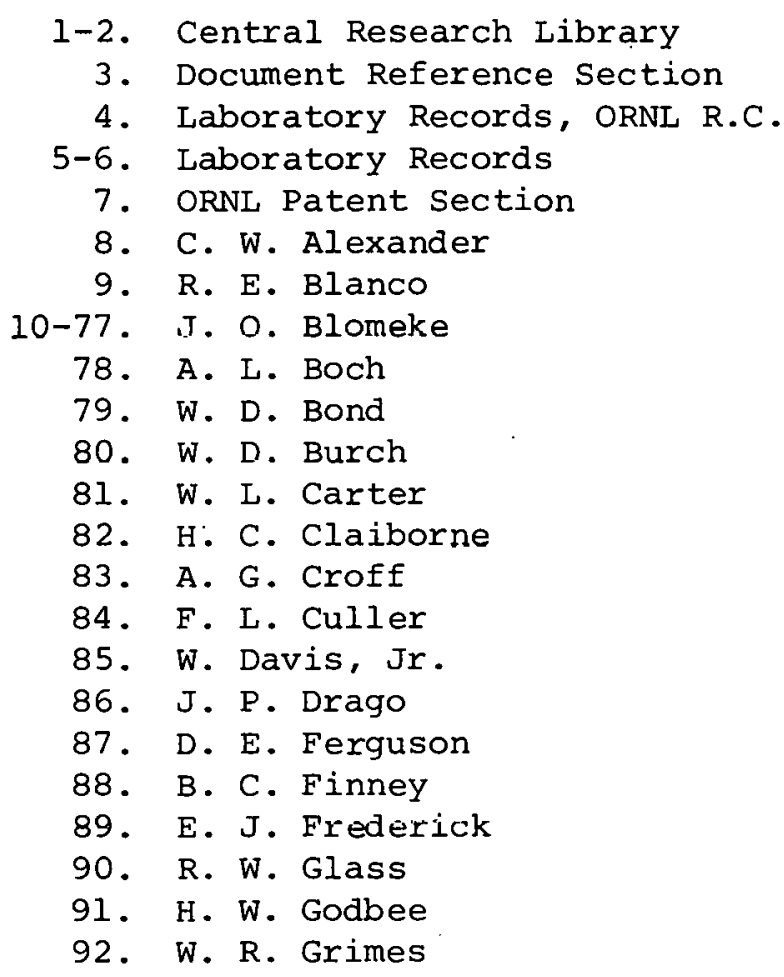

93. G. H. Jenks

94. C. W. Kee

95. A. H. Kibbey

96. E. Lamb

97. R. E. Leuze

98. K. H. Lin

99. R. S. Lowrie

100. J. F. MeBride

101. W. C. MCClain

102. L. E. McNeese

103. J. G. Moore

104. J. M. Morrison

105. K. J. Notz

106. C. D. Scott

107. D. W. Tedder

108. D. B. Trauger

109. B. L. Vondra

110. R. G. Wymer

111. C. D. Zerby

112. W. K. Davis (consultant)

113. E. L. Gaden, Jr. (consultant)

114. C. H. Ice (consultant)

115. R. B. Richards (consultant)

EXTERNAL DISTRIBUTION

116-142. Technical Information Center, Oak Ridge, TN 37830

ERDA-ORO, Oak Ridge, TN 37830

143. Research and Technical Support Division

144. E. II. Hardison

145. J, J. Schreiher

U.S. Energy Research and Develupment Administration, Washington, DC 20545

146. C. B. Bastin

147. D. G. Boyer

148. W. K. Eister

149. O. P. Gormley

150. R. W. Ramsey, Jr.

151. R. A. Wolfe

U.S. Nuclear Regulatory Commission, Washington, DC 20555

152. C. B. Bartlett

153. W. P. Bishop

154. R. M. Bernero 
Battelle Northwest, P. O. Box 999, Richland, WA 99352

155. R. M. Fleischman

156. R. W. McKee

157. A. M. Platt

158. L. D. Williams

Exxon Nuclear, 2101 Horn Rapids Road, Richland, WA 99352

159. L. T. Lakey

Allied-General Nuclear Services, P. O. Box 847, Barnwell, SC 29812

160. A. K. Williams

Argonne National Laboratory, 9700 South Cass Ave., Argonne, IL 62439

161. M. J. Steindler

Hanford Engineering Development Laboratory, P. O. Box 1970, Richland, WA 99352

162. G. L. Richardson

Atlantic Richfield Hanford Company, P. O. Box 250, Richland, WA 99352 163. D. D. Wodrich

U.S. Energy Research and Development Administration, Richland Operations Office, P. O. Box 550, Richland, WA 99352

1G4.. R. B. Fnranson

Sandia Laboratories, P. O. Box 5800, Albuquerque, NM 87115

165. P. D. O'Brien

Nuclear.Fuel Services, Inc., P. O. Box 124, West Valley, NY 14171

166. J. P. Duckworth

The University of Arizona, Department of Nuclear Engineering, Tuscon, AZ 85721

167. R. G. Post

Science Applications, Inc., 1600 Anderson Road, McLean, VA 22101

168. G. Bray

Allied Chemical Corp., P. O. Box 2204, Idaho Falls, ID 83401

169. B. C. Musgrave

George C. Marshall space Flight Center, Marshall Space Flight Center, AL 35812

170. R. E. Burns 
The University of California, Department of Nuclear Engineering, Berkeley, CA 94720

171. T. H. Pigford

General Electric Company, 175 Curtner Ave., San Jose, CA 95100

172. A. B. Carson

Georgia Institute of Technology, Department of Nuclear Engineering, Atlanta, GA 30332

173. Alfred Schneider

Hahn-Meitner-Institut für Kernforschung Berlin, Sektor Kernchemie, Glienicker Strasse 100, I Berlin 39, Germany

174. Hans W. Levi

Centre d'Etudes Nucleaires, Fontenay-aux-Roses, F-92, Chatillonsous-Bagneux, France

175. Y. J. Sousselier

Nuclear Research Center Karlsruhe, Postfach 3640 , D-75 Karlsruhe, Federal Republic of Germany

176. H. Krause

British Nuclear Fuels, Ltd., Risley, Warrington, Lancashire, England 177. D. W. Clelland 